Review

\title{
Coordination chemistry of the main group elements with phosphine, arsine and stibine ligands
}

\author{
Jennifer Burt, William Levason*, Gillian Reid \\ School of Chemistry, University of Southampton, Southampton SO17 1BJ, UK
}

\section{Contents}

1. Introduction

2. Bonding....

3. Characterisation techniques

4. s-Block complexes

5. Group 12 .....

5.1. Zinc .

5.2. Cadmium

5.3. Mercury.

6. Group 13 .....

6.1. Boron...

6.2. Aluminium

6.3. Gallium

6.4. Indium

6.5. Thallium

7. Group $14 \ldots .$.

7.1. Silicon.

7.2. Germanium.

7.3. Tin....

7.4. Lead.

8. Group 15

8.1. Phosphorus.

8.2. Arsenic.....

8.3. Antimony .

8.4. Bismuth....

9. Group 16 ....

10. Applications and outlook.

Acknowledgements.

References

\section{A R T I C L E I N F O}

\section{Article history:}

Received 13 August 2013

Accepted 29 September 2013

Available online 10 October 2013

\section{Keywords:}

Phosphine

Arsine

\begin{abstract}
A B S T R A C T
Complexes of Group 2, 12, 13, 14, 15 and 16 elements with mono-, bi-, and poly-dentate phosphine and arsine ligands (and including the very few examples of stibine and bismuthine donor ligands) are described. Polydentate ligand complexes containing neutral or charged $\mathrm{N}, \mathrm{O}, \mathrm{C}$, or S donor groups in addition to phosphino or arsino donor groups are included, but charged $\mathrm{P}$ or As (phosphides, arsenides, phosphinomethanides etc.) ligands are excluded. Emphasis is placed upon the X-ray structures, multinuclear NMR data and reactions. The major differences of this class of complexes compared to the familiar d-block phosphine/arsine complexes are discussed and rationalised in terms of the E-M bonding
\end{abstract}

Abbreviations: E, P, As, Sb or Bi; M, Lewis acid atom usually metal or metalloid; X, F, Cl, Br or I; R, alkyl or aryl; DMPP, tris(2,6-dimethoxyphenyl)phosphine; TMPP, tris(2,4,6-

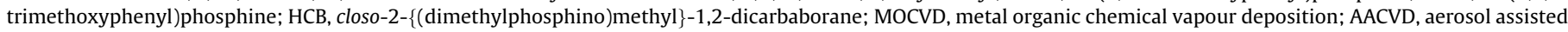
chemical vapour deposition.

* Corresponding author. Tel.: +44 2380593792; fax: +44 2380593781.

E-mail address: wxl@soton.ac.uk (W. Levason). 
Stibine

Main group complexes

${ }^{31} \mathrm{P}$ NMR

$\mathrm{X}$-ray structures models. Literature coverage is focussed on the last 20 years, although key older work is also included where necessary for comparison purposes, and the article includes work published up to early 2013.

\section{Introduction}

Phosphine and arsine ligands have continued to play a major role in transition metal (d-block) coordination and organometallic chemistry over several decades, forming a wide range of complexes with different metal oxidation states [1-3], and transition metal phosphine complexes are also widely used in catalysis [4]. The weaker donor stibines have a more restricted chemistry and relatively few bismuthine complexes are known [5]. These Group 15 neutral donor ligands are described in standard texts as $\pi$-acids or $\sigma$-donor $\pi$-acceptor ligands, which bond to d-block metals by $\sigma$-donation of the lone pair on the pnictogen (E) centre, supplemented in many cases by $\pi$-acceptance of d-electron density into E-C $\sigma^{*}$ - (or a combination of E-C $\sigma^{*}$ - and empty E d-) orbitals. The relative importance of the $\sigma$ and $\pi$ components varies with the metal oxidation state and d-electron density [6]. Main group elements also form complexes with phosphines and arsines, and more rarely with stibines, although these have received much less research effort than the d-block analogues, and have fundamentally different properties. This article reviews recent research on the synthesis and properties of phosphine, arsine and stibine complexes of the s- and p-block metals, metalloids and non-metals, specifically complexes of Groups 2,12,13,14,15 and 16 elements. The area has been reviewed twice before $[7,8]$, the last article having been published in 1995, and thus we will concentrate on work published in the last 20 years, although the fragmented nature of the field necessitates some reference to older work. Group 12 complexes have sometimes been included in reviews of transition metal phosphines [9] and refs therein. Phosphine boranes have been reviewed twice in the recent past $[10,11]$ and thus are not included in the present article. A number of weak adducts between $\mathrm{PH}_{3}$ or $\mathrm{PMe}_{3}$ and p-block Lewis acids formed at low temperatures and characterised using matrix isolation techniques have been discussed in a very recent comprehensive review of the field [12].

The present article describes the complexes of mono-, bi- and multi-dentate phosphine, arsine, stibine and bismuthine ligands, and includes examples (but not exhaustive coverage) of hybrid ligands where other donors, mostly neutral or charged $\mathrm{O}-, \mathrm{N}-$ or Sgroups are incorporated in the ligand. Coverage here is focused on complexes of interest from the point of view of the pnictogen ligand. Charged pnictogen ligands such as phosphides, arsenides, phosphinomethanides etc. are not included in this article. Literature coverage is on work published up to early 2013.

\section{Bonding}

The traditional $\sigma$-donor $\pi$-acceptor bonding model [6] used for d-block metal phosphine complexes needs to be revised for p-block adducts. In the p-block the Lewis acid has ns and three np orbitals available for $\sigma$ bonding, the filled $(n-1) d$ orbitals are essentially core orbitals and unavailable for $\pi$-donation, and the nd orbitals are empty and too high in energy to make any significant contributions to bonding. Thus the Lewis acid-phosphine(arsine) bond must be based upon $\sigma$-donation alone (this is also true for $\mathrm{d}^{0}$ transition metal acceptors such as $\mathrm{Ti}(\mathrm{IV})$ or $\mathrm{Nb}(\mathrm{V})$ which form stable complexes with phosphine and arsine ligands). For compounds with four electron pair donor ligands ( $8 \mathrm{e}^{-}$systems), the traditional model using either $\mathrm{sp}^{3}$ hybridisation or symmetry allowed MO's derived from the central atom ns and $3 \mathrm{np}$ orbitals is adequate and this is used, for example, for four coordinate $\mathrm{Cd}(\mathrm{II})$ or $\mathrm{Hg}$ (II)

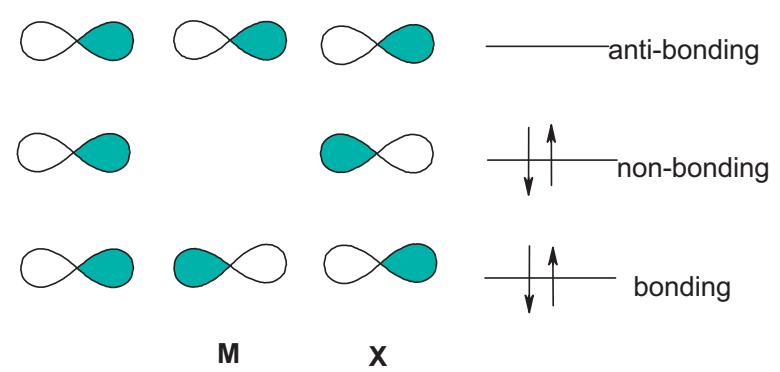

(a).

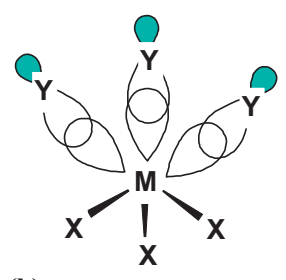

(b).

Fig. 1. (a) 3c-4e bonding model. (b) M-Y $\sigma$-bonding using $M-X \sigma^{*}$ orbitals as acceptor orbitals.

adducts. However, the unavailability of d orbitals for bonding raises problems when the central atom exceeds an octet of electrons in its valence shell. This hypervalency is common in p-block chemistry and requires a different bonding model. The popular model currently used to describe such bonding is the 3c-4e bond [13-18].

For a linear $\mathrm{X}-\mathrm{M}-\mathrm{X}$ unit, the central $\mathrm{M}$ uses one empty np orbital, and combining this with one filled donor orbital on each $\mathrm{X}$ results in three molecular orbitals: bonding, non-bonding and antibonding (in order of increasing energy). The two donor electron pairs are then placed in the first two MOs (Fig. 1(a)). For an octahedron this model uses three orthogonal p orbitals on $M$ to form three $3 \mathrm{c}-4 \mathrm{e}$ bonds. When the groups around the central $M$ are different $(\mathrm{X}-\mathrm{M}-\mathrm{Y})$, the model describes an $\mathrm{M}-\mathrm{X}$ bond with the bonding orbital polarised towards $\mathrm{X}$, and the $\mathrm{M}-\mathrm{X}$ antibonding orbital $\left(\sigma^{*}\right)$ polarised towards $M$. The $M-X \sigma^{*}$ orbital is empty and can therefore act as an acceptor orbital towards an electron pair from Y (Fig. 1(b)). If the $\mathrm{X}-\mathrm{M}$ bonding dominates (primary bond) and $\mathrm{M}-\mathrm{Y}$ is weak the latter is often termed "secondary bonding".

It is also possible to use a mixture of the models. For example, for a trigonal bipyramid, $\mathrm{sp}^{2}$ hybrid orbitals can be used for the equatorial $\mathrm{MX}_{3}$ donors (conventional 2c-2e bonding), while the $\mathrm{p}_{z}$ orbital is used for the axial $\mathrm{X}-\mathrm{M}-\mathrm{X}$ unit (3c-4e). However, for cases where the coordination number of the central atom exceeds six, which is the case in many crown ether complexes for example, problems of insufficient orbitals for covalent bonding remain, and currently such high coordination numbers lack a generally satisfactory detailed model.

A second issue in main group chemistry is that of lone pairs, which arise when the p-block element is in its $(n-2)$ oxidation state, e.g. As(III) or Ge(II). The "extra" electron pair may be stereochemically active whereby it occupies one of the central element's "bonding" orbitals, or it may be stereochemically inactive, in which case it is assumed to occupy the spherically symmetric ns orbital (which is then unavailable for bonding to ligands). Similarly in the $3 c-4 e$ bonding model, a stereochemically active lone pair occupies 
Table 1

Nuclear properties.

\begin{tabular}{|c|c|c|c|c|c|c|}
\hline Isotope & N.A. $(\%)^{a}$ & $I$ & $\gamma\left(\operatorname{radT^{-1}} \mathrm{s}^{-1}\right)^{\mathrm{b}}$ & $Q / 10^{-28} \mathrm{~m}^{2 \mathrm{c}}$ & $\mathrm{Rc}^{\mathrm{d}}$ & Reference \\
\hline${ }^{1} \mathrm{H}$ & 99.9 & $1 / 2$ & 26.75 & - & 5680 & $\mathrm{SiMe}_{4}$ \\
\hline${ }^{13} \mathrm{C}$ & 1.1 & $1 / 2$ & 6.73 & - & 1 & $\mathrm{SiMe}_{4}$ \\
\hline${ }^{9} \mathrm{Be}$ & 100 & $3 / 2$ & -3.76 & $5.3 \times 10^{-2}$ & 78.8 & $\mathrm{Be}\left(\mathrm{NO}_{3}\right)_{2}$ aq. \\
\hline${ }^{25} \mathrm{Mg}$ & 10.1 & $5 / 2$ & -1.64 & 0.22 & 1.54 & $\mathrm{MgCl}_{2}$ aq. \\
\hline${ }^{67} \mathrm{Zn}$ & 4.1 & $5 / 2$ & 1.67 & 0.15 & 0.66 & $\mathrm{Zn}\left(\mathrm{ClO}_{4}\right)_{2}$ aq. \\
\hline${ }^{113} \mathrm{Cd}$ & 12.3 & $1 / 2$ & -5.93 & - & 7.7 & $\mathrm{CdMe}_{2}$ \\
\hline${ }^{199} \mathrm{Hg}$ & 16.8 & $1 / 2$ & 4.85 & - & 5.68 & $\mathrm{HgMe}_{2}$ or $\mathrm{Hg}\left(\mathrm{ClO}_{4}\right)_{2}$ aq. \\
\hline${ }^{11} \mathrm{~B}$ & 80.4 & $3 / 2$ & 8.59 & $4.1 \times 10^{-2}$ & 754 & $\mathrm{BF}_{3}\left(\mathrm{Et}_{2} \mathrm{O}\right)$ \\
\hline${ }^{27} \mathrm{Al}$ & 100 & $5 / 2$ & 6.98 & 0.14 & 1170 & {$\left[\mathrm{Al}\left(\mathrm{H}_{2} \mathrm{O}\right)_{6}\right]^{3+}$} \\
\hline${ }^{71} \mathrm{Ga}$ & 39.6 & $3 / 2$ & 8.18 & 0.11 & 322 & {$\left[\mathrm{Ga}\left(\mathrm{H}_{2} \mathrm{O}\right)_{6}\right]^{3+}$} \\
\hline${ }^{115} \mathrm{In}$ & 95.7 & $9 / 2$ & 5.89 & 0.86 & 1920 & {$\left[\mathrm{In}\left(\mathrm{H}_{2} \mathrm{O}\right)_{6}\right]^{3+}$} \\
\hline${ }^{205} \mathrm{Tl}$ & 70.5 & $1 / 2$ & 15.69 & - & 807 & $\mathrm{Tl}\left(\mathrm{NO}_{3}\right) \mathrm{aq}$. \\
\hline${ }^{29} \mathrm{Si}$ & 4.67 & $1 / 2$ & -5.32 & - & 2.1 & $\mathrm{SiMe}_{4}$ \\
\hline${ }^{73} \mathrm{Ge}$ & 7.8 & $9 / 2$ & -0.94 & -0.17 & 0.62 & $\mathrm{GeMe}_{4}$ \\
\hline${ }^{119} \mathrm{Sn}$ & 8.6 & $1 / 2$ & -10.03 & - & 25.7 & $\mathrm{SnMe}_{4}$ \\
\hline${ }^{207} \mathrm{~Pb}$ & 22.6 & $1 / 2$ & 5.62 & - & 11.9 & $\mathrm{PbMe}_{4}$ \\
\hline${ }^{31} \mathrm{P}$ & 100 & $1 / 2$ & 10.83 & - & 377 & $\mathrm{H}_{3} \mathrm{PO}_{4}$ \\
\hline${ }^{75}$ As & 100 & $3 / 2$ & 4.59 & 0.29 & 144 & $\mathrm{KAsF}_{6}$ \\
\hline${ }^{121} \mathrm{Sb}$ & 57.3 & $5 / 2$ & 6.44 & -0.33 & 520 & {$\left[\mathrm{SbCl}_{6}\right]^{-}$} \\
\hline${ }^{209} \mathbf{B i}$ & 100 & $9 / 2$ & 4.38 & -0.37 & 819 & $\mathrm{KBiF}_{6}$ \\
\hline${ }^{77} \mathrm{Se}$ & 7.6 & $1 / 2$ & 5.12 & - & 3.02 & $\mathrm{SeMe}_{2}$ \\
\hline${ }^{125} \mathrm{Te}$ & 6.99 & $1 / 2$ & -8.51 & - & 12.8 & $\mathrm{TeMe}_{2}$ \\
\hline${ }^{19} \mathrm{~F}$ & 100 & $1 / 2$ & 25.18 & - & 4730 & $\mathrm{CFCl}_{3}$ \\
\hline
\end{tabular}

Data taken from $[19,20]$. Where more than one NMR active isotope exists, only the preferred one is listed.

a Natural abundance.

b Magnetogyric ratio $\times 10^{7}$.

c Quadrupole moment.

d Receptivity relative to ${ }^{13} \mathrm{C}$.

the "non-bonding" orbital. If the lone pair occupies the ns orbital, it exerts a screening effect and weakens the M-L bonding. There are considerable ranges of behaviour across the p-block; in particular the difference between "primary" and "secondary" coordination for an X-M-Y system may be very clear in some cases, but almost lost in others, and one should recognise the limitations of simple bonding models.

Developments in computational techniques have resulted in much effort devoted to Lewis acid-Lewis base behaviour. Unsurprisingly, the focus of most of this work has been on the lighter elements where more accurate basis sets are available, and thus nitrogen and oxygen ligands have received far more attention than phosphines, with arsenic ligands rarely considered even in passing. Whilst these studies have revealed the complexity of the factors involved and their subtle interplay, many of the conclusions remain controversial even for individual systems. References to recent studies of phosphine complexes appear in the appropriate sections below.

\section{Characterisation techniques}

The s- and p-block phosphine and arsine complexes are typically diamagnetic pale-coloured solids, with varying degrees of sensitivity towards atmospheric dioxygen or water. In solution at ambient temperatures, even in non-coordinating solvents, they may partially dissociate the neutral ligand, or undergo fast ligand exchange. These properties present challenges to the various characterisation techniques available, and the relatively slow development of the field in earlier years was not unconnected with the difficulties of characterising the complexes with the existing techniques.

Single crystal X-ray diffraction is the pre-eminent technique for characterising complexes of the main group elements in the solid state. The developments over the past 20 years, both in computing and in fast X-ray data acquisition, which have made data collection at low temperatures routine and data solution rapid, have been a major driver in this area. Only precise X-ray structural data can provide certainty over metal complex identity, geometry and dimensions, which underpin the bonding models and the overall understanding in this area. Spectroscopic techniques - IR/Raman and solid state NMR spectroscopy - provide useful support, not least in confirming (or not) that the X-ray structural data obtained on a single crystal is representative of the bulk material. IR/Raman data provide evidence for the presence of the phosphine/arsine in the complex, but rarely anything more, although they are invaluable at establishing the absence of $\mathrm{P}=\mathrm{O}$ or $\mathrm{As}=\mathrm{O}$ vibrations (seen when adventitious oxidation of the ligand occurs). Solid state NMR spectra can be obtained readily from ${ }^{31} \mathrm{P}\left\{{ }^{1} \mathrm{H}\right\}$ in phosphine complexes (but not for ${ }^{75} \mathrm{As}$ in corresponding arsines (see below)), and may be supplemented by studies of the metal nucleus if it has an isotope with suitable nuclear properties. However, whilst there is a considerable amount of data on some systems, such as on mercuryphosphines, SS NMR use is very patchy across the p-block. Where data have been reported, they are often linked to crystallographic data (identifying the symmetry inequivalent phosphorus sites in the solid) or compared to corresponding solution data, rather than being used as a "stand alone" probe for structural characterisation.

In solution, NMR spectroscopy is the major tool since all complexes are diamagnetic. For alkyl substituted ligands (less clearly for aryl analogues), ${ }^{1} \mathrm{H}$ and ${ }^{13} \mathrm{C}\left\{{ }^{1} \mathrm{H}\right\}$ NMR spectroscopy provide evidence of the coordination of the phosphine or arsine ligand, usually via high frequency coordination shifts ( $\left.\Delta=\delta_{\text {complex }}-\delta_{\text {ligand }}\right)$ in the resonances, whilst the number of resonances reflect the number of inequivalent ligand sites. Most useful is ${ }^{31} \mathrm{P}$ NMR spectroscopy which monitors the donor atom itself, where coordination has the greatest effect. Usually coordination results in high frequency shifts in the phosphorus resonance, although there are a number of cases where the coordination shift $(\Delta)$ is near zero, or to low frequency (i.e. negative $\Delta$ ), and the underlying reasons are disputed. Examples of these erratic coordination shifts are found for $\mathrm{Ga}(\mathrm{III})$ and In(III) complexes (q.v.). The only naturally occurring nucleus of arsenic $\left({ }^{75} \mathrm{As}\right)$ is quadrupolar (see Table 1$)$ and resonances are unobservably broad in the low symmetry environments found in metal arsine complexes. The nuclei of antimony and bismuth are also quadrupolar and unobservable in low symmetry environments. Due in many cases to ligand exchange processes, it is necessary to record NMR spectra over a range of temperatures-typically $295-180 \mathrm{~K}$ (the 


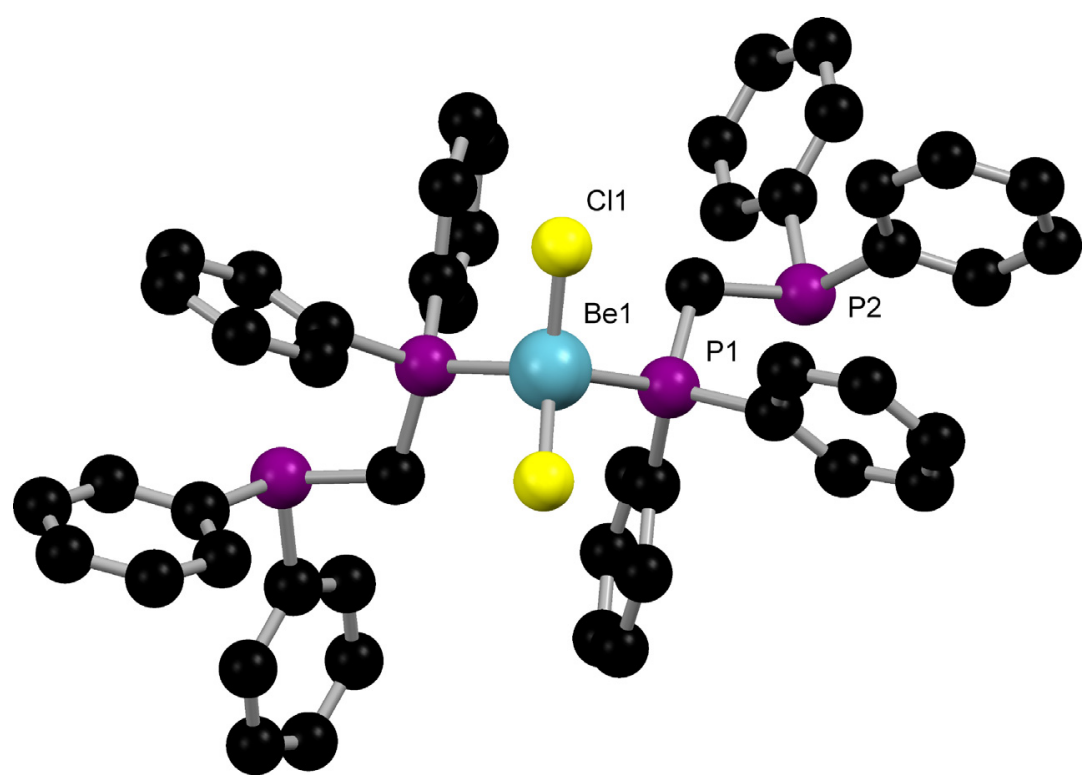

Fig. 2. View of the structure of $\left[\mathrm{BeCl}_{2}\left(\kappa^{1}-\mathrm{Ph}_{2} \mathrm{PCH}_{2} \mathrm{PPh}_{2}\right)_{2}\right]$ redrawn from Ref. [21].

widely used solvent, $\mathrm{CH}_{2} \mathrm{Cl}_{2}$, melts at $176 \mathrm{~K}$ ), and VTNMR can, in favourable cases, be used to explore these exchange processes. Spin-spin couplings are also only observed when intermolecular ligand exchange is slow, again often requiring low temperature studies.

Table 1 lists the nuclear properties of the s- and p-block elements in this article $[19,20]$. As can be seen, all the elements have at least one NMR active nucleus. In the absence of chemical problems such as fast exchange on the NMR timescale, all the $I=1 / 2$ nuclei can be observed relatively easily (all have a greater receptivity than ${ }^{13} \mathrm{C}$, although ${ }^{29} \mathrm{Si}$ and ${ }^{119} \mathrm{Sn}$ have long $\mathrm{T}_{1}$ 's which can be problematic). The quadrupolar nuclei are a more mixed batch, ${ }^{9} \mathrm{Be}$ and ${ }^{11} \mathrm{~B}$ have small Q's and are easily observed, ${ }^{27} \mathrm{Al}$ and ${ }^{71} \mathrm{Ga}$ can be observed if the electric field gradient is sufficiently small, whereas in practice ${ }^{115} \mathrm{In}$ is only seen in close to cubic symmetry environments. ${ }^{25} \mathrm{Mg}$, ${ }^{67} \mathrm{Zn}$ and ${ }^{73} \mathrm{Ge}$ all have unfavourable properties: in addition to being quadrupolar, they have low absolute frequencies and poor natural abundance, and although all have been observed in certain high symmetry cases, they are generally not likely to be useful.

\section{4. s-Block complexes}

The affinity of the hard Groups 1 and 2 ions for soft phosphine ligands would be expected to be small, and isolated complexes are limited to a few examples containing Be or $\mathrm{Mg}$. The first complex of a beryllium halide was $\left[\mathrm{BeCl}_{2}\left(\kappa^{1}-\mathrm{Ph}_{2} \mathrm{PCH}_{2} \mathrm{PPh}_{2}\right)_{2}\right]$, made as colourless crystals from $\mathrm{BeCl}_{2}$ and the diphosphine in $\mathrm{CH}_{2} \mathrm{Cl}_{2}$. The structure (Fig. 2) shows a distorted tetrahedral molecule [21]. The dinuclear $\left[\mathrm{Be}_{2} \mathrm{Cl}_{2}(\mu-\mathrm{Cl})_{2}\left(\mathrm{PCy}_{3}\right)_{2}\right]$ (Fig. 3) was made unexpectedly from $\left[\mathrm{Pd}\left(\mathrm{PCy}_{3}\right)_{2}\right]$ and $\mathrm{BeCl}_{2}$ in hot toluene, the other product being metallic Pd [22]. The only other phosphine examples are the long known $\left[\mathrm{BePh}_{2}\left(\mathrm{PMe}_{3}\right)_{2}\right][23]$ and [ $\left.\mathrm{BeMe}_{2}\left(\mathrm{PMe}_{3}\right)_{2}\right]$, studied in situ by ${ }^{1} \mathrm{H}$ and ${ }^{9} \mathrm{Be}$ NMR spectroscopy in solution [24]. It seems that there is considerable scope for developing the phosphine chemistry of beryllium. As in other areas of Be chemistry, workers seem to have been deterred by the toxicity.

Both $\mathrm{PMe}_{3}$ and $\mathrm{Me}_{2} \mathrm{P}\left(\mathrm{CH}_{2}\right)_{2} \mathrm{PMe}_{2}$ form colourless crystalline adducts with [ $\left.\left(\eta^{5}-\mathrm{C}_{5} \mathrm{H}_{5}\right)_{2} \mathrm{Mg}\right]$ [25]. The presence of Mg-P bonds in $\left[\mathrm{Mg}\left\{\mathrm{C}_{6} \mathrm{H}_{3}-2,6-\left(\mathrm{CH}_{2} \mathrm{PMe}_{2}\right)_{2}\right\}_{2}\right]$, made from the lithium salt and $\mathrm{MgCl}_{2}$ in diethyl ether solution, has been established crystallographically. The structure (Fig. 4) shows an octahedral magnesium(II) ion coordinated to four phosphine groups and mutually trans arylcarbons (Mg-C = 2.26(1), Mg-P=2.770(1), 2.761(1) $\AA$ ) [26]. Calcium forms complexes with various anionic phosphorus ligands, but no complexes with phosphines are known [27].

\section{Group 12}

Phosphine and arsine complexes of mercury(II) and cadmium(II) have been known for many years, although the development of the chemistry of corresponding zinc complexes is more recent. Their chemistry was often included in reviews on transition metal complexes ([10] and refs therein), and the earlier work on $\mathrm{Hg}$ is detailed in reviews of the coordination and structural chemistry of the element [28,29]. However, since the M(II) complexes contain $\mathrm{d}^{10}$ metal centres they also fit within the scope of the present article, and since no recent reviews exist, they are discussed below.

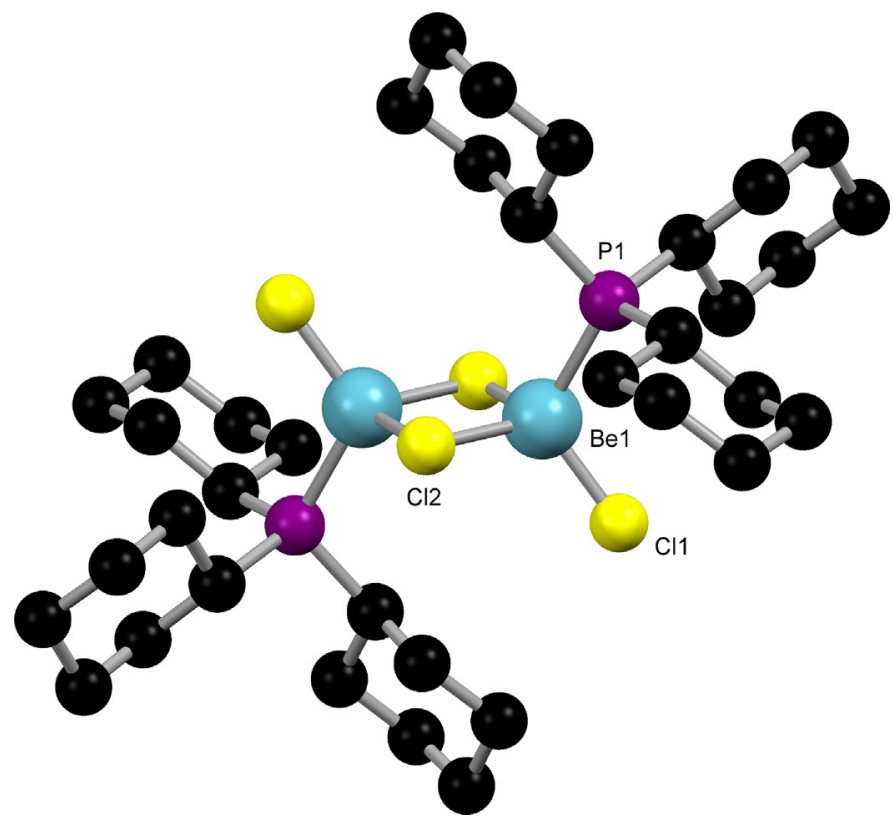

Fig. 3. View of the structure of $\left[\mathrm{Be}_{2} \mathrm{Cl}_{2}(\mu-\mathrm{Cl})_{2}\left(\mathrm{PCy}_{3}\right)_{2}\right]$ redrawn from Ref. [22]. 
Table 2

$\mathrm{X}$-ray structural data on zinc complexes.

\begin{tabular}{|c|c|c|c|}
\hline Complex & Structure & $\mathrm{Zn}-\mathrm{P} / \mathrm{As}(\AA)$ & Refs. \\
\hline$\left[\mathrm{ZnCl}_{2}\left(\mathrm{PPh}_{3}\right)_{2}\right]$ & Tetrahedral & $2.498(3), 2.611(3)$ & [33] \\
\hline$\left[\mathrm{ZnCl}_{2}\left(\mathrm{PMe}_{3}\right) 2\right]$ & Tetrahedral & $2.259(8), 2.384(1)$ & [30] \\
\hline$\left.\left[\mathrm{Zn}\left(\mathrm{PMe}_{3}\right)_{3}(\mu-\mathrm{Cl}) \mathrm{ZnCl}\right]_{3}\right]$ & Tetrahedral & $2.359(1)-2.391(1)$ & [30] \\
\hline$\left[\mathrm{ZnCl}_{3}\left(\mathrm{PPh}_{3}\right)\right]^{-}$ & Tetrahedral & $2.392(1)$ & [32] \\
\hline$\left[\mathrm{ZnBr}_{3}\left(\mathrm{PPh}_{3}\right)\right]^{-}$ & Tetrahedral & $2.429(5)$ & [31] \\
\hline$\left[\mathrm{ZnI}\left(\mathrm{PEt}_{3}\right)(\mu-\mathrm{I})_{2} \mathrm{ZnI}\left(\mathrm{PEt}_{3}\right)\right]$ & Tetrahedral dimer & $2.361(5)$ & [36] \\
\hline$\left[\mathrm{ZnI}\left\{\mathrm{P}\left(\mathrm{NMe}_{2}\right)_{3}\right\}(\mu-\mathrm{I})_{2} \mathrm{ZnI}\left\{\mathrm{P}\left(\mathrm{NMe}_{2}\right)_{3}\right\}\right]$ & Tetrahedral dimer & $2.372(3)$ & [37] \\
\hline$\left[\mathrm{ZnI}_{2}\left(\mathrm{PPh}_{2} \mathrm{Me}\right)_{2}\right]$ & Tetrahedral & $2.416(4)$ & [37] \\
\hline$\left[\mathrm{ZnI}_{2}\left(\mathrm{AsMe}_{3}\right)_{2}\right]$ & Tetrahedral & $2.445(8)-2.493(7)$ & [38] \\
\hline$\left[\mathrm{ZnI}\left(\mathrm{AsEt}_{3}\right)(\mu-\mathrm{I})_{2} \mathrm{ZnI}\left(\mathrm{AsEt}_{3}\right)\right]$ & Tetrahedral dimer & $2.446(3)$ & [38] \\
\hline$\left[\mathrm{Zn}_{2} \mathrm{Cl}_{2}\left\{\mu-\mathrm{P}\left(\mathrm{SiMe}_{3}\right)_{2}\right\}_{2}\left(\mathrm{P}^{i} \mathrm{Pr}_{3}\right)_{2}\right]$ & Dimer & $2.430(1), 2.408(1)^{\phi}$ & [39] \\
\hline$\left[\mathrm{Zn}_{2} \mathrm{Cl}_{2}\left\{\mu-\mathrm{As}\left(\mathrm{SiMe}_{3}\right)_{2}\right\}_{2}\left(\mathrm{P}^{i} \mathrm{Pr}_{3}\right)_{2}\right]$ & Dimer & $2.399(2), 2.413(2)$ & [39] \\
\hline$\left[\mathrm{Zn}_{10} \mathrm{Cl}_{12}\left(\mathrm{PSiMe}_{3}\right)_{4}\left(\mathrm{P}^{i} \mathrm{Pr}_{3}\right)_{4}\right]$ & & $2.383(2)^{\phi}$ & [39] \\
\hline$\left[\mathrm{Zn}_{10} \mathrm{Cl}_{12}\left(\mathrm{AsSiMe}_{3}\right)_{4}\left(\mathrm{P}^{i} \mathrm{Pr}_{3}\right)_{4}\right]$ & & $2.238(4)$ & [39] \\
\hline$\left[\mathrm{ZnI}\left\{\mathrm{As}\left(\mathrm{SiMe}_{3}\right)_{3}\right\}(\mu-\mathrm{I})_{2} \mathrm{ZnI}\left\{\mathrm{As}\left(\mathrm{SiMe}_{3}\right)_{3}\right\}\right]$ & Dimer & $2.447(1), 2.458(1)$ & [39] \\
\hline$\left[\mathrm{ZnI}\left\{\mathrm{P}\left(\mathrm{SiMe}_{3}\right)_{3}\right\}(\mu-\mathrm{I})_{2} \mathrm{ZnI}\left\{\mathrm{P}\left(\mathrm{SiMe}_{3}\right)_{3}\right\}\right]$ & Dimer & $2.391(5), 2.396(5)$ & {$[40]$} \\
\hline$\left[\mathrm{Zn}_{10} \mathrm{Cl}_{12}\left(\mathrm{PSiMe}_{3}\right)_{4}\left(\mathrm{PEt}_{2} \mathrm{Ph}\right)_{4}\right]$ & & $2.375(2)^{\phi}$ & {$[40]$} \\
\hline$\left[\mathrm{Zn}_{4}\left(\mathrm{PPh}_{2}\right)_{4} \mathrm{Cl}_{4}\left(\mathrm{PMe}^{n} \mathrm{Pr}_{2}\right)_{2}\right]$ & & $2.403(3), 2.426(3)^{\phi \varphi}$ & {$[41]$} \\
\hline$\left[\mathrm{Zn}_{8} \mathrm{Se}(\mathrm{SePh})_{14}\left(\mathrm{P}^{n} \mathrm{Pr}_{3}\right)_{2}\right]$ & & $2.380(2)$ & [42] \\
\hline$\left[\mathrm{Zn}_{10} \mathrm{Te}_{4}(\mathrm{TePh})_{12}\left(\mathrm{P}^{n} \mathrm{Pr}_{3}\right)_{2}\right]$ & & $2.428(4)$ & {$[42]$} \\
\hline$\left[\mathrm{Zn}_{12} \mathrm{Te}_{9}(\mathrm{TeMes})_{6}\left(\mathrm{Ph}_{2} \mathrm{PCH}_{2} \mathrm{PPh}_{2}\right)_{4}\right]$ & & $2.464(5)-2.521(5)$ & [43] \\
\hline$\left[\mathrm{Zn}\left(\mathrm{PMe}_{3}\right)\left(\mathrm{Et}_{2} \mathrm{NCS}_{2}\right)_{2}\right]$ & 5-Coordinate & $2.379(4)-2.388(3)$ & {$[44]$} \\
\hline$\left[\left\{\mathrm{Zn}\left(\mathrm{Et}_{2} \mathrm{NCS}_{2}\right)_{2}\right\}_{2}\left\{\boldsymbol{\mu}-\mathrm{Et}_{2} \mathrm{P}\left(\mathrm{CH}_{2}\right)_{2} \mathrm{PEt}_{2}\right\}\right]$ & & $2.388(2)$ & [45] \\
\hline$\left[\mathrm{Zn}\left(\mathrm{S}-{ }^{\mathrm{t}} \mathrm{Bu}_{3} \mathrm{C}_{6} \mathrm{H}_{2}\right)_{2}\left(\mathrm{PMePh}_{2}\right)\right]$ & 3-Coordinate & $2.414(4)$ & [46] \\
\hline$\left[\mathrm{Zn}_{2}\left(\mu-\mathrm{O}-2,6-\mathrm{F}_{2} \mathrm{C}_{6} \mathrm{H}_{3}\right)_{2}\left(\mathrm{O}-2,6-\mathrm{F}_{2} \mathrm{C}_{6} \mathrm{H}_{3}\right)_{2}\left(\mathrm{PC}_{3}\right)_{2}\right]$ & Dimer & $2.401(4), 2.419(4)$ & {$[48]$} \\
\hline$\left[\mathrm{Zn}\left(\mathrm{O}-{ }^{t} \mathrm{Bu}_{3} \mathrm{C}_{6} \mathrm{H}_{2}\right)_{2}\left(\mathrm{PMe}_{3}\right)_{2}\right]$ & Tetrahedral & $2.542(3), 2.588(3)$ & [49] \\
\hline$\left[\mathrm{Zn}\left(\mathrm{CH}_{2} \mathrm{CH}_{2} \mathrm{CH}_{2} \mathrm{PPh}_{2}\right)_{2}\right]$ & Tetrahedral & $2.572(3), 2.598(3)$ & {$[55]$} \\
\hline$\left[\mathrm{Zn}\left\{\mathrm{SC}_{6} \mathrm{H}_{3}\left(\mathrm{SiMe}_{3}\right) \mathrm{PPh}_{2}\right\}_{2}\right]$ & Tetrahedral & $2.387(1)$ & [56] \\
\hline$\left[\mathrm{ZnCl}_{2}\left\{\mathrm{Fe}\left(\mathrm{C}_{5} \mathrm{H}_{4} \mathrm{P}^{i} \mathrm{Pr}_{2}\right)_{2}\right\}\right]$ & Tetrahedral & $2.4141(6), 2.4165(6)$ & [52] \\
\hline$\left[\mathrm{ZnI}_{2}\left\{\mathrm{Ph}_{2} \mathrm{P}\left(\mathrm{CH}_{2}\right)_{2} \mathrm{PPh}_{2}\right\}\right]_{4}$ & Helical tetramer & $2.399(2)$ & {$[51]$} \\
\hline$\left[\mathrm{ZnCl}_{2}\left\{\mathrm{Ph}_{2} \mathrm{P}\left(\mathrm{CH}_{2}\right)_{2} \mathrm{PPh}_{2}\right\}\right]_{n}$ & Polymer & $2.4534(8), 2.4113(9)$ & {$[51]$} \\
\hline$\left[\mathrm{ZnCl}_{2}\left(4,6\right.\right.$-bis $\left(3-{ }^{i} \mathrm{Pr}_{2} \mathrm{PC}_{6} \mathrm{H}_{4}\right)$ dibenzofuran $\left.)\right]$ & & $2.416(1), 2.420(1)$ & [54] \\
\hline$\left[\mathrm{ZnCl}_{2}\left\{\mathrm{Fe}\left(\mathrm{C}_{5} \mathrm{H}_{4} \mathrm{PPh}_{2}\right)\left(\mathrm{C}_{5} \mathrm{H}_{4}-2 \mathrm{py}\right)\right\}\right]$ & P,N chelate & $2.444(1), 2.462(1)$ & [58] \\
\hline$\left[\mathrm{ZnBr}_{2}\left\{\mathrm{Fe}\left(\mathrm{C}_{5} \mathrm{H}_{4} \mathrm{PPh}_{2}\right)\left(\mathrm{C}_{5} \mathrm{H}_{4}-2 \mathrm{py}\right)\right\}\right]$ & $\mathrm{P}, \mathrm{N}$ chelate & $2.428(1)$ & {$[58]$} \\
\hline$\left[\mathrm{ZnBr}_{2}\left\{\mathrm{Fe}\left(\mathrm{C}_{5} \mathrm{H}_{4} \mathrm{PPh}_{2}\right)\left(\mathrm{C}_{5} \mathrm{H}_{4} \mathrm{CH}_{2}-2 \mathrm{py}\right)\right\}\right]$ & P,N chelate & $2.439(1)$ & [58] \\
\hline$\left[\left\{\mathrm{ZnBr}_{2}\left\{\boldsymbol{\mu}-\mathrm{Fe}\left(\mathrm{C}_{5} \mathrm{H}_{4} \mathrm{PPh}_{2}\right)\left(\mathrm{C}_{5} \mathrm{H}_{4} \mathrm{CH}_{2}-2 \mathrm{py}\right)\right\}\right\}_{2}\right]$ & Cyclic dimer & $2.455(2)$ & [58] \\
\hline$\left[\mathrm{ZnBr}_{2}\left\{\mathrm{Fe}\left(\mathrm{C}_{5} \mathrm{H}_{4} \mathrm{PPh}_{2}\right)\left(\mathrm{C}_{5} \mathrm{H}_{4}-3 \mathrm{py}\right)\right\}\right]$ & Polymer & $2.463(2)$ & {$[58]$} \\
\hline$\left[\mathrm{Zn}_{2} \mathrm{~L}_{2} \mathrm{Cl}_{3}\right]\left[\mathrm{ClO}_{4}\right]\left(\mathrm{L}^{\prime}=\mathrm{Ph}_{2} \mathrm{PCH}_{2} \mathrm{CH}_{2} \mathrm{CH}_{2}-1,4,7\right.$-triazacyclononane & & $2.518(4), 2.524(4)$ & [59] \\
\hline$\left[\mathrm{Zn}\left\{\mathrm{P}\left(2-\mathrm{C}_{6} \mathrm{H}_{4} \mathrm{PPh}_{2}\right)_{2}\right\}_{2}\right]$ & Trigonal planar & $2.463(2)^{\phi}$ & {$[60]$} \\
\hline
\end{tabular}

$\phi=\mathrm{d}(\mathrm{Zn}-$ phosphine $)$.

\subsection{Zinc}

Zinc(II) halides typically form four-coordinate distorted tetrahedral complexes $\left[\mathrm{ZnX}_{2}\left(\mathrm{PR}_{3}\right)_{2}\right]$ or $\left[\mathrm{ZnX}\left(\mathrm{PR}_{3}\right)(\mu-\mathrm{X})_{2} \mathrm{ZnX}\left(\mathrm{PR}_{3}\right)\right]$ (Table 2), or rarely $\left[\mathrm{ZnX}_{3}\left(\mathrm{PR}_{3}\right)\right]^{-}(\mathrm{X}=\mathrm{Cl}, \mathrm{Br}$ or $\mathrm{I})$. There is also

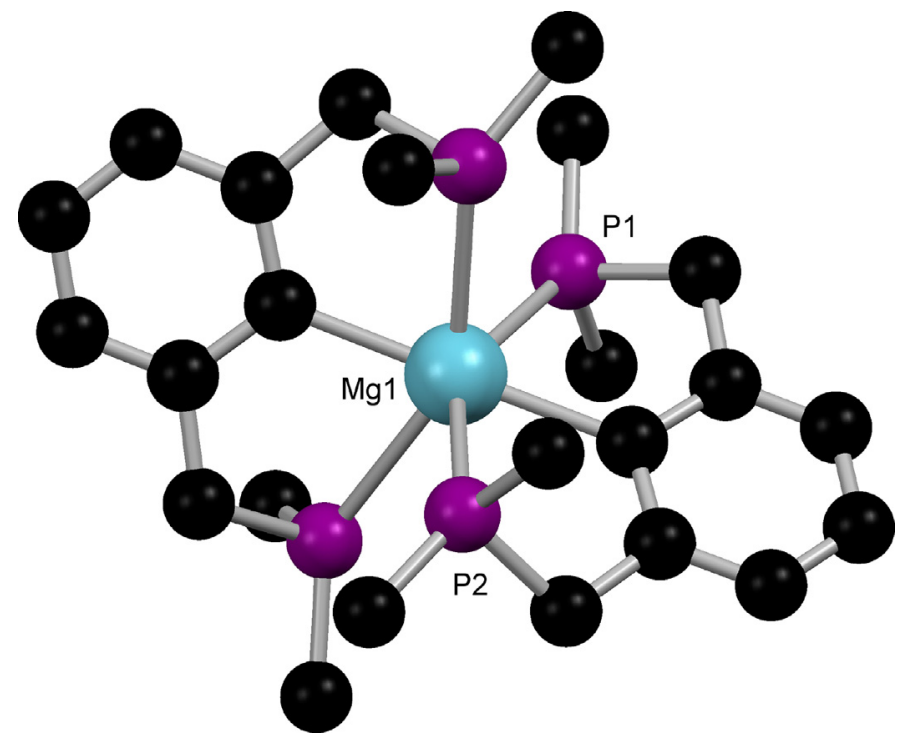

Fig. 4. View of the structure of $\left[\mathrm{Mg}\left\{\mathrm{C}_{6} \mathrm{H}_{3}-2,6-\left(\mathrm{CH}_{2} \mathrm{PMe}_{2}\right)_{2}\right\}_{2}\right]$ redrawn from Ref. [26]. a unique $\left[\mathrm{Zn}\left(\mathrm{PMe}_{3}\right)_{3}(\mu-\mathrm{Cl}) \mathrm{ZnCl}_{3}\right]$ complex in which a threecoordinate $\mathrm{Zn}\left(\mathrm{PMe}_{3}\right)_{3}$ group links via a single chloride bridge to a $\left[\mathrm{ZnCl}_{3}\right]^{-}$anion (Fig. 5) [30]. Few anionic $\left[\mathrm{ZnX}{ }_{3}\left(\mathrm{PR}_{3}\right)\right]^{-}$species are known, probably more due to the lack of attempts to prepare them than inherent instability, but two have been structurally characterised [31,32]. Direct reaction of zinc halides with phosphines in a 1:2 molar ratio in diethyl ether, ethanol or THF solution usually results in the formation of $\left[\mathrm{ZnX}_{2}\left(\mathrm{PR}_{3}\right)_{2}\right](\mathrm{R}=\mathrm{Me}, \mathrm{Ph}, p$ $\left.\mathrm{MeC}_{6} \mathrm{H}_{4}, p-\mathrm{MeOC}_{6} \mathrm{H}_{4}\right)[30,33-35]$, although $\mathrm{P}\left(p-\mathrm{ClC}_{6} \mathrm{H}_{4}\right)_{3}$ gave only $1: 1$ complexes, and $\mathrm{P}\left(o-\mathrm{MeC}_{6} \mathrm{H}_{4}\right)_{3}$ or $\mathrm{P}\left(o-\mathrm{MeOC}_{6} \mathrm{H}_{4}\right)_{3}$ failed to complex at all with $\mathrm{ZnBr}_{2}$ [34].

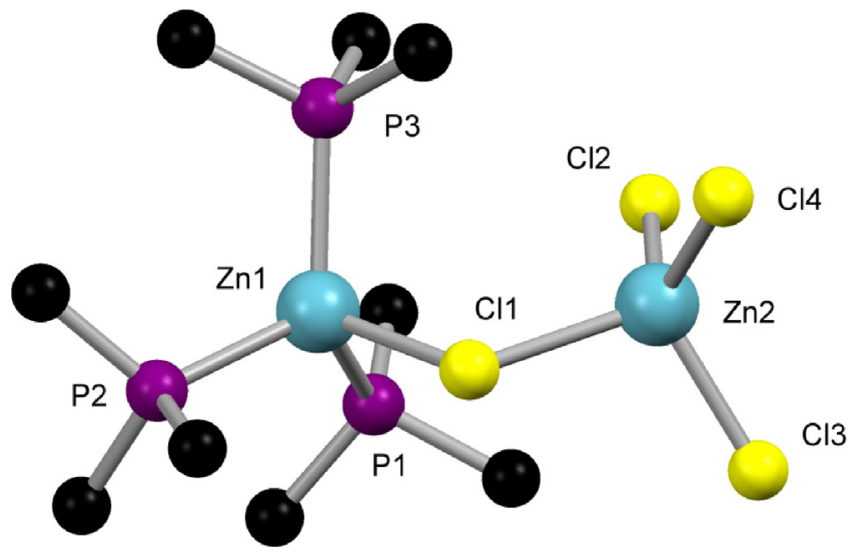

Fig. 5. View of the structure of $\left[\mathrm{Zn}\left(\mathrm{PMe}_{3}\right)_{3}(\mu-\mathrm{Cl}) \mathrm{ZnCl}_{3}\right]$ redrawn from Ref. [30]. 


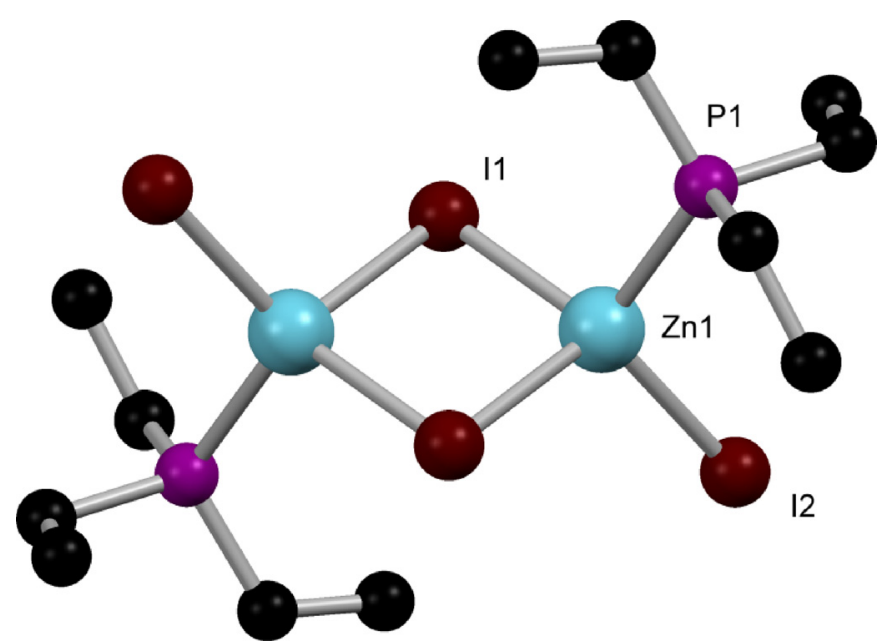

Fig. 6. View of the structure of $\left[\mathrm{Zn}_{2} \mathrm{I}_{2}(\mu-\mathrm{I})_{2}\left(\mathrm{PEt}_{3}\right)_{2}\right]$ redrawn from Ref. [36].

An alternative synthetic approach is the reaction of zinc powder with $\mathrm{R}_{3} \mathrm{PI}_{2}$, which in most cases forms the $1: 1$ adducts $\left[\mathrm{Zn}_{2} \mathrm{I}_{2}(\mu\right.$ $\left.\mathrm{I})_{2}\left(\mathrm{PR}_{3}\right)_{2}\right]\left(\mathrm{R}=\mathrm{Me}\right.$, Et, ${ }^{n} \mathrm{Pr},{ }^{i} \mathrm{Pr},{ }^{n} \mathrm{Bu}, \mathrm{Cy}, \mathrm{CH}_{2} \mathrm{Ph}, p-\mathrm{FC}_{6} \mathrm{H}_{4}, p-\mathrm{ClC}_{6} \mathrm{H}_{4}$, p- $\mathrm{MeOC}_{6} \mathrm{H}_{4}, 2,6-(\mathrm{MeO})_{2} \mathrm{C}_{6} \mathrm{H}_{3}, 2,4,6-(\mathrm{MeO})_{3} \mathrm{C}_{6} \mathrm{H}_{2}, \mathrm{NMe}_{2}, \mathrm{NEt}_{2}$, $\left.\mathrm{CH}_{2} \mathrm{CH}_{2} \mathrm{Ph}, \mathrm{CH}_{2} \mathrm{CH}_{2} \mathrm{CN} ; \mathrm{R}_{3}=\left(\mathrm{CH}_{2} \mathrm{Ph}\right) \mathrm{Ph}_{2},\left(\mathrm{C}_{5} \mathrm{H}_{4} \mathrm{~N}\right) \mathrm{Ph}_{2}\right)$, the iodobridged dimer structure being established for $\left[\mathrm{Zn}_{2} \mathrm{I}_{2}(\mu-\mathrm{I})_{2}\left(\mathrm{PEt}_{3}\right)_{2}\right]$ (Fig. 6) and for $\left[\mathrm{Zn}_{2} \mathrm{I}_{2}(\mu-\mathrm{I})_{2}\left\{\mathrm{P}\left(\mathrm{NMe}_{2}\right)_{3}\right\}_{2}\right][36,37]$. Curiously the reaction of $\mathrm{R}_{3} \mathrm{PI}_{2}\left(\mathrm{R}_{3}=\mathrm{Ph}_{3}, \mathrm{Ph}_{2} \mathrm{Me}\right.$ or $\left.\mathrm{Ph}_{2} \mathrm{Et}\right)$ with zinc powder gave only equimolar mixtures of $\left[\mathrm{ZnI}_{2}\left(\mathrm{PR}_{3}\right)_{2}\right]$ and $\mathrm{ZnI}_{2}$ [37]. It would seem that the stoichiometry of the complexes obtained is not simply controlled by the cone angle of the phosphine and the ratio of reagents used, but is a result of both steric and electronic factors and possibly crystal packing or $\pi$-stacking effects [37]. In solution, ${ }^{31} \mathrm{P}\left\{{ }^{1} \mathrm{H}\right\}$ NMR spectroscopy suggests the complexes retain their identity and do not give mixtures of $1: 1$ and $1: 2$ complexes.

The corresponding reactions of the appropriate $\mathrm{R}_{3} \mathrm{AsI}_{2}$ with zinc powder produced $\left[\mathrm{ZnI}_{2}\left(\mathrm{AsMe}_{3}\right)_{2}\right]$ (equimolar mixture with $\mathrm{ZnI}_{2}$ ) and $\left[\mathrm{Zn}_{2} \mathrm{I}_{2}(\mu-\mathrm{I})_{2}\left(\mathrm{AsEt}_{3}\right)_{2}\right]$, respectively [38]; it seems unlikely that steric factors alone could account for the different structures formed here either.

The silyl-phosphine and -arsine, $\mathrm{E}\left(\mathrm{SiMe}_{3}\right)_{3}(\mathrm{E}=\mathrm{P}$ or As), react with $\mathrm{ZnI}_{2}$ to give $\left[\mathrm{Zn}_{2} \mathrm{I}_{2}(\mu-\mathrm{I})_{2}\left\{\mathrm{E}\left(\mathrm{SiMe}_{3}\right)_{3}\right\}_{2}\right][39,40]$. Meanwhile the reaction of $\mathrm{E}\left(\mathrm{SiMe}_{3}\right)_{3}\left(\mathrm{E}=\mathrm{P}\right.$ or As) with $\mathrm{ZnCl}_{2}$ in the presence of $\mathrm{Pn}^{n} \mathrm{Pr}_{3}$ leads to the cleavage of an E-Si link and formation of $\left[\mathrm{Zn}_{2} \mathrm{Cl}_{2}\left\{\mu-\mathrm{E}\left(\mathrm{SiMe}_{3}\right)_{2}\right\}_{2}\left(\mathrm{P}^{\mathrm{n}} \mathrm{Pr}_{3}\right)_{2}\right]$ [39]. Fenske and co-workers have described a range of zinc clusters with PR, AsR, SeR or TeR bridges and terminal phosphine groups (Table 2) [39-43].

Zinc phosphine complexes with various anions in place of halides have been prepared, including examples with $\mathrm{R}_{3} \mathrm{Si}^{-}, \mathrm{R}_{3} \mathrm{Sn}^{-}$, $\mathrm{RO}^{-}, \mathrm{R}_{2} \mathrm{NCS}_{2}^{-}, \mathrm{RS}^{-}, \mathrm{RSe}^{-}$and $\mathrm{RTe}^{-}$; structurally authenticated examples are listed in Table 2 [35,44-49]. Although most contain four-coordinate zinc, the dithiocarbamate complexes are fivecoordinate and $\left[\mathrm{Zn}\left(\mathrm{S}^{-}{ }^{t} \mathrm{Bu}_{3} \mathrm{C}_{6} \mathrm{H}_{2}\right)_{2}\left(\mathrm{PMePh}_{2}\right)\right]$ is three-coordinate. Applications of zinc phosphine complexes in coupling or polymerisation reactions of $\mathrm{CO}_{2}$ and ethylene oxide or cyclohexene oxide have been described [48-50].

Diffusion of a $\mathrm{CH}_{2} \mathrm{Cl}_{2}$ solution of $\mathrm{Ph}_{2} \mathrm{P}\left(\mathrm{CH}_{2}\right)_{2} \mathrm{PPh}_{2}$ into $\mathrm{H}_{2} \mathrm{O} / \mathrm{EtOH}$ solutions of $\mathrm{ZnX}_{2}$ results in diphosphine bridged polymers [51]. The $\left[\mathrm{ZnI}_{2}\left\{\mathrm{Ph}_{2} \mathrm{P}\left(\mathrm{CH}_{2}\right)_{2} \mathrm{PPh}_{2}\right\}\right]_{\mathrm{n}}$ contains helices with a pitch of four zinc atoms, whereas $\left[\mathrm{ZnX}_{2}\left\{\mathrm{Ph}_{2} \mathrm{P}\left(\mathrm{CH}_{2}\right)_{2} \mathrm{PPh}_{2}\right\}\right]_{n}(\mathrm{X}=\mathrm{Cl}$ or $\mathrm{Br})$ are linear chain polymers. In contrast, in $\left[\mathrm{ZnCl}_{2}\left\{\mathrm{Fe}\left(\mathrm{C}_{5} \mathrm{H}_{4} \mathrm{P}^{\mathrm{i}} \mathrm{Pr}_{2}\right)_{2}\right\}\right]$ the diphosphine chelates to the zinc [52] and $\left[\mathrm{ZnCl}_{2}\left\{\mathrm{Fe}\left(\mathrm{C}_{5} \mathrm{H}_{4} \mathrm{PCy}_{2}\right)_{2}\right\}\right]$ is likely to have a similar structure [53]. Chelation is also present in [ $\mathrm{ZnCl}_{2}$ (4,6-bis $\left(3-{ }^{i} \mathrm{Pr}_{2} \mathrm{PC}_{6} \mathrm{H}_{4}\right)$ dibenzofuran)] [54].

Phosphine donor groups are found with several bidentate anionic ligands. $\left[\mathrm{Zn}\left(\mathrm{CH}_{2} \mathrm{CH}_{2} \mathrm{CH}_{2} \mathrm{PPh}_{2}\right)_{2}\right]$ contains a distorted

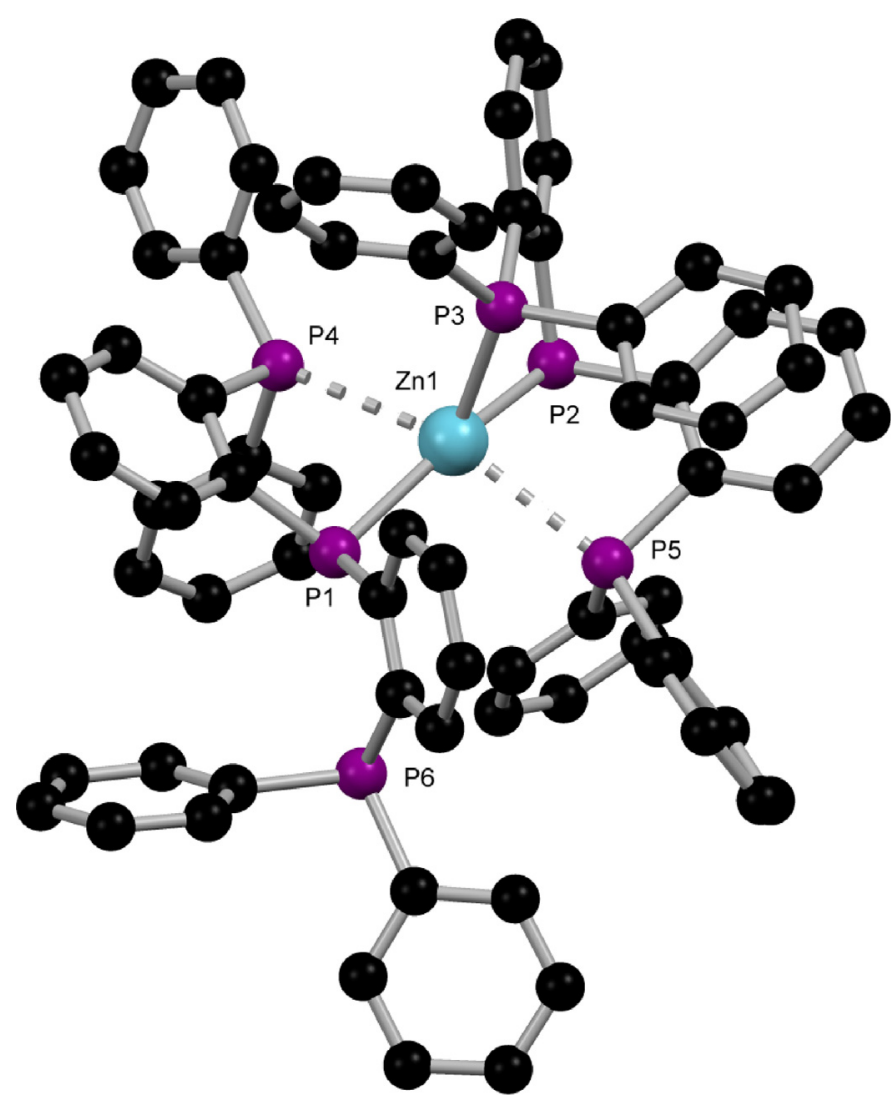

Fig. 7. View of the structure of $\left[\mathrm{Zn}\left\{\mathrm{P}\left(2-\mathrm{C}_{6} \mathrm{H}_{4} \mathrm{PPh}_{2}\right)_{2}\right\}_{2}\right]$ redrawn from Ref. [60].

tetrahedral geometry with chelating 3-diphenylphosphinopropyl groups [55]. Electrochemical oxidation of a zinc anode in MeCN solutions of various phosphinothiols $\left(o-\mathrm{C}_{6} \mathrm{H}_{4}\left(\mathrm{PPh}_{2}\right) \mathrm{SH}\right.$, $o-\mathrm{C}_{6} \mathrm{H}_{3}\left(\mathrm{Me}_{3} \mathrm{Si}\right)\left(\mathrm{PPh}_{2}\right) \mathrm{SH}$ or $\left.\mathrm{PhP}\left(o-\mathrm{C}_{6} \mathrm{H}_{4} \mathrm{SH}\right)_{2}\right)$ gave the $\mathrm{Zn}(\mathrm{II})$ complexes of the phosphinothiolate anions [56].

Other hybrid ligand complexes include $\left[\mathrm{ZnL}\left(\mathrm{O}_{3} \mathrm{SCF}_{3}\right)_{2}\right](\mathrm{L}=2,6$ $\left.\left(\mathrm{Ph}_{2} \mathrm{PCH}_{2} \mathrm{CH}_{2} \mathrm{SCH}_{2}\right)_{2} \mathrm{C}_{5} \mathrm{H}_{3} \mathrm{~N}\right)$ [57], and various ferrocene based pyridylphosphines [58]. The complexes of the latter group all contain tetrahedral zinc and include chelate monomers, bridged dimers and chain polymers. In $\left[\mathrm{Zn}_{2} \mathrm{~L}_{2}^{\prime} \mathrm{Cl}_{3}\right]\left[\mathrm{ClO}_{4}\right]$ $\left(\mathrm{L}^{\prime}=\mathrm{Ph}_{2} \mathrm{PCH}_{2} \mathrm{CH}_{2} \mathrm{CH}_{2}\right.$-1,4,7-triazacyclononane) one zinc centre is $5-\left(\mathrm{N}_{3} \mathrm{PCl}\right)$ the second six-coordinate $\left(\mathrm{N}_{3} \mathrm{PCl}_{2}\right)$ [59]. The tridentate ligand $\mathrm{HP}\left(2-\mathrm{C}_{6} \mathrm{H}_{4} \mathrm{PPh}_{2}\right)_{2}$ deprotonates on reaction with $\mathrm{ZnEt}_{2}$ or $\mathrm{Zn}\left\{\mathrm{N}\left(\mathrm{SiMe}_{3}\right)_{2}\right\}_{2}$ to form $\left[\mathrm{Zn}\left\{\mathrm{P}\left(2-\mathrm{C}_{6} \mathrm{H}_{4} \mathrm{PPh}_{2}\right)_{2}\right\} \mathrm{R}\right](\mathrm{R}=\mathrm{Et}$ or $\left.\mathrm{N}\left(\mathrm{SiMe}_{3}\right)_{2}\right)$ [60]. The latter slowly decomposes in solution, forming $\left[\mathrm{Zn}\left\{\mathrm{P}\left(2-\mathrm{C}_{6} \mathrm{H}_{4} \mathrm{PPh}_{2}\right)_{2}\right\}_{2}\right]$ whose structure (Fig. 7) shows zinc coordinated to two phosphido and one phosphino groups, with very long contacts $(\sim 2.8 \AA)$ to two further phosphino donors. Solution ${ }^{31} \mathrm{P}\left\{{ }^{1} \mathrm{H}\right\}$ NMR data suggest fast exchange between the phosphino groups at ambient temperatures.

\subsection{Cadmium}

The common types of cadmium phosphine complexes, $\left[\mathrm{CdX}{ }_{2}\left(\mathrm{PR}_{3}\right)_{2}\right]$ and $\left[\mathrm{CdX}\left(\mathrm{PR}_{3}\right)(\mu-\mathrm{X})_{2} \mathrm{CdX}\left(\mathrm{PR}_{3}\right)\right]$ were established seventy years ago, and only a small amount of new work has appeared. The crystal structures of distorted tetrahedral $\left[\mathrm{CdI}_{2}\left(\mathrm{PPh}_{3}\right)_{2}\right](\mathrm{Cd}-\mathrm{P}=2.631(2), 2.653(1) \AA)$ and $\left[\mathrm{CdI}_{2} \mathrm{~L}_{2}^{\prime \prime}\right]\left(\mathrm{L}^{\prime \prime}=1\right.$ phenyldibenzophosphole $(\mathrm{Cd}-\mathrm{P}=2.616(3), 2.603(3) \AA)$ have been determined [61]. Cadmium has two relatively sensitive NMR nuclei in ${ }^{111} \mathrm{Cd}(I=1 / 2,12.8 \%, \mathrm{Rc}=6.9)$ and ${ }^{113} \mathrm{Cd}(I=1 / 2,12.3 \%$, $\mathrm{Rc}=7.7$ ) and several studies of cadmium phosphines by combined ${ }^{113} \mathrm{Cd} /{ }^{31} \mathrm{P}\left\{{ }^{1} \mathrm{H}\right\}$ NMR spectroscopy have been reported. In 
Table 3

Illustrative ${ }^{31} \mathrm{P},{ }^{113} \mathrm{Cd}$ and ${ }^{199} \mathrm{Hg}$ NMR data for phosphine complexes.

\begin{tabular}{|c|c|c|c|c|c|c|}
\hline Complex & $\delta\left({ }^{31} \mathrm{P}\right)^{\mathrm{a}}$ & ${ }^{1} J\left({ }^{113} \mathrm{Cd}-{ }^{31} \mathrm{P}\right)(\mathrm{Hz})^{\mathrm{a}}$ & $\delta\left({ }^{31} \mathrm{P}\right)^{\mathrm{b}}$ & ${ }^{1} J\left({ }^{113} \mathrm{Cd}-{ }^{31} \mathrm{P}\right)(\mathrm{Hz})^{\mathrm{b}}$ & $\delta\left({ }^{113} \mathrm{Cd}\right)^{\mathrm{c}}$ & ${ }^{1} J\left({ }^{113} \mathrm{Cd}-{ }^{31} \mathrm{P}\right)(\mathrm{Hz})^{\mathrm{c}}$ \\
\hline$\left[\mathrm{CdCl}_{2}\left(\mathrm{PEt}_{3}\right)_{2}\right]$ & -0.8 & 1677 & $2.5,4.5$ & 1543,1504 & 632 & 1520 \\
\hline$\left[\mathrm{CdBr}_{2}\left(\mathrm{PEt}_{3}\right)_{2}\right]$ & -3.8 & 1547 & 0.5 & $1430^{\mathrm{d}}$ & 606 & 1471 \\
\hline$\left[\mathrm{CdI}_{2}\left(\mathrm{PEt}_{3}\right)_{2}\right]$ & -10.7 & 1367 & -6.8 & $1333^{\mathrm{d}}$ & 531 & 1327 \\
\hline$\left[\mathrm{Cd}_{2} \mathrm{Cl}_{4}\left(\mathrm{PEt}_{3}\right)_{2}\right]$ & & & 8.0 & 2504 & 412 & 2503 \\
\hline$\left[\mathrm{Cd}_{2} \mathrm{Br}_{4}\left(\mathrm{PEt}_{3}\right)_{2}\right]$ & & & 3.2 & 2355 & 341 & 2356 \\
\hline \multirow[t]{2}{*}[\mathrm{Cd}_{2}\mathrm{I}_{4}(\mathrm{PEt}_{3})_{2}]{} & & & 1.5 & $1768^{\mathrm{d}}$ & 319 & 1782 \\
\hline & $\delta\left({ }^{31} \mathrm{P}\right)^{\mathrm{a}}$ & ${ }^{1} J\left({ }^{199} \mathrm{Hg}^{31} \mathrm{P}\right)(\mathrm{Hz})^{\mathrm{a}}$ & $\delta\left({ }^{31} \mathrm{P}\right)^{\mathrm{b}}$ & $\delta\left({ }^{199} \mathrm{Hg}\right)^{\mathrm{e}}$ & ${ }^{1} J\left({ }^{199} \mathrm{Hg}^{31} \mathrm{P}\right)(\mathrm{Hz})^{\mathrm{e}}$ & \\
\hline$\left[\mathrm{HgCl}_{2}\left(\mathrm{P}^{n} \mathrm{Bu}_{3}\right)_{2}\right]$ & 30.0 & 5100 & $30.1,35.1$ & 1831 & 5100 & \\
\hline$\left[\mathrm{HgBr}_{2}\left(\mathrm{P}^{n} \mathrm{Bu}_{3}\right)_{2}\right]$ & 23.0 & 4750 & & 1796 & 4750 & \\
\hline$\left[\mathrm{HgI}_{2}\left(\mathrm{P}^{n} \mathrm{Bu}_{3}\right)_{2}\right]$ & 12.1 & 4100 & & 1541 & 4090 & \\
\hline$\left[\mathrm{Hg}_{2} \mathrm{Cl}_{4}\left(\mathrm{P}^{n} \mathrm{Bu}_{3}\right)_{2}\right]$ & 34.2 & 7500 & $37.2,38.2$ & 1486 & 7500 & \\
\hline$\left[\mathrm{Hg}_{2} \mathrm{Br}_{4}\left(\mathrm{P}^{n} \mathrm{Bu}_{3}\right)_{2}\right]$ & 27.6 & 6650 & $34.4,36.3$ & 1241 & 6650 & \\
\hline$\left[\mathrm{Hg}_{2} \mathrm{I}_{4}\left(\mathrm{P}^{n} \mathrm{Bu}_{3}\right)_{2}\right]$ & 6.3 & 5150 & $19.4,22.2$ & 515 & 5130 & \\
\hline
\end{tabular}

a $\mathrm{CH}_{2} \mathrm{Cl}_{2}$ solution relative to $\mathrm{H}_{3} \mathrm{PO}_{4}$.

b CP MAS.

c $\mathrm{CP}$ MAS relative to $\mathrm{Cd}\left(\mathrm{ClO}_{4}\right)_{2} \cdot 6 \mathrm{H}_{2} \mathrm{O}$.

d Averaged values ${ }^{1} J\left({ }^{111} \mathrm{Cd} /{ }^{113} \mathrm{Cd}-{ }^{31} \mathrm{P}\right)$.

e $\mathrm{CH}_{2} \mathrm{Cl}_{2}$ solution relative to 0.1 molar $\mathrm{Hg}\left(\mathrm{ClO}_{4}\right)_{2}$ in $\mathrm{HClO}_{4}$.

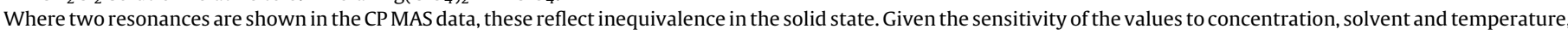
the data quoted sometimes can differ significantly from those obtained by other workers. Data taken from $[61,62,69,70]$.

solution at room temperature the resonances are often broad and $111 / 113 \mathrm{Cd}-{ }^{31} \mathrm{P}$ couplings poorly resolved, indicative of dynamic exchange processes. The lines sharpen and couplings become clearer on cooling the solutions as the rate of exchange slows. Whilst full details of the NMR data are best obtained by consulting the original reports, there are some well-established trends clearly evident for most systems [62]. In the ${ }^{31} \mathrm{P}\left\{{ }^{1} \mathrm{H}\right\}$ NMR spectra, for comparable complexes, the magnitude of the coordination shift $(\Delta)$ and the ${ }^{1} J\left({ }^{111 / 113} \mathrm{Cd}-{ }^{31} \mathrm{P}\right)$ decrease $\mathrm{Cl}>\mathrm{Br}>\mathrm{I}$ (see Table 3 ). The ${ }^{113} \mathrm{Cd}$ chemical shifts are sensitive to temperature and changes in halide, moving to low frequency with increase in size and decrease in electronegativity; they are somewhat less sensitive to changes in the phosphine. Although the spectra are often more complicated due to lower symmetry, the same trends are observed in the CP MAS NMR spectra of the solid complexes. A recent study [61] examined $\left[\mathrm{CdX}_{2}\left(\mathrm{PR}_{3}\right)_{2}\right]\left(\mathrm{X}=\mathrm{Cl}, \mathrm{Br}, \mathrm{I} ; \mathrm{R}=\mathrm{Et},{ }^{n} \mathrm{Bu}\right.$, $\mathrm{Ph}, 1$-phenyldibenzophosphole, 1-phenyl-3,4-dimethylphosphole) and $\left[\mathrm{Cd}_{2} \mathrm{X}_{4}\left(\mathrm{PEt}_{3}\right)_{2}\right]$ by both solution and solid (CP MAS) NMR spectroscopy, and concluded that the dimer was in equilibrium with $\mathrm{CdX}_{2}$ and $\left[\mathrm{CdX}_{2}\left(\mathrm{PEt}_{3}\right)_{2}\right]$ in solution. Compounds of stoichiometry $\left[\mathrm{Cd}_{2} \mathrm{X}_{4}\left(\mathrm{P}^{n} \mathrm{Bu}_{3}\right)_{3}\right](\mathrm{X}=\mathrm{Cl}, \mathrm{Br}$ or $\mathrm{I})$ were also isolated. Solution NMR studies suggested these were completely dissociated into $\left[\mathrm{Cd}_{2} \mathrm{X}_{4}\left(\mathrm{P}^{n} \mathrm{Bu}_{3}\right)_{2}\right]$ and $\left[\mathrm{CdX}_{2}\left(\mathrm{P}^{n} \mathrm{Bu}_{3}\right)_{2}\right]$, but in the solid state they appear to be single discrete species $\left[\left({ }^{n} \mathrm{Bu}_{3} \mathrm{P}\right)_{2} \mathrm{Cd}(\mu\right.$ $\left.\mathrm{X})_{2} \mathrm{CdX}_{2}\left(\mathrm{P}^{n} \mathrm{Bu}_{3}\right)\right]$ with four- and five-coordinate cadmium.

The complexes $\left[\mathrm{Cd}_{2} \mathrm{Br}_{2}(\mu-\mathrm{Br})_{2}\left\{\mathrm{P}\left(\mathrm{SiMe}_{3}\right)_{3}\right\}_{2}\right]$ and $\left[\mathrm{Cd}_{2} \mathrm{I}_{2}(\mu-\right.$ $\left.\mathrm{I})_{2}\left\{\mathrm{E}\left(\mathrm{SiMe}_{3}\right)_{3}\right\}_{2}\right] \quad(\mathrm{E}=\mathrm{P}$, As) have been isolated [39,40], and under other conditions various clusters form including $\left[\mathrm{Cd}_{2} \mathrm{Br}_{2}\left\{\mathrm{P}\left(\mathrm{SiMe}_{3}\right)_{2}\right\}_{2}\left(\mathrm{P}^{n} \mathrm{Pr}_{3}\right)_{2}\right], \quad\left[\mathrm{Cd}_{10} \mathrm{Br}_{12}\left\{\mathrm{PSiMe}_{3}\right)_{4}\left\{\mathrm{P}\left(\mathrm{SiMe}_{3}\right)_{3}\right\}_{4}\right]$ and $\left[\mathrm{Cd}_{10} \mathrm{I}_{12}\left\{\mathrm{AsSiMe}_{3}\right)_{4}\left\{\mathrm{As}\left(\mathrm{SiMe}_{3}\right)_{3}\right\}_{4}\right]$. Other clusters are the adamantanoid $\left[(\mu-\mathrm{SR})_{6} \mathrm{Cd}_{4}\left(\mathrm{PPh}_{3}\right)_{2}\left(\mathrm{ClO}_{4}\right)_{2}\right], \quad[(\mu-$ $\left.\mathrm{SeR})_{6} \mathrm{Cd}_{4}\left(\mathrm{PPh}_{3}\right)_{2}\left(\mathrm{ClO}_{4}\right)_{2}\right]$ [63] and $\left[\mathrm{Cd}_{10} \mathrm{E}_{4}\left(\mathrm{E}^{\prime} \mathrm{Ph}\right)_{12}\left(\mathrm{PR}_{3}\right)_{4}\right](\mathrm{E}$, $\mathrm{E}^{\prime}=\mathrm{Te}$, Se, sometimes S) [64]; in all of these species the phosphines occupy terminal vertices. Mixed ligand complexes, generally similar to their zinc analogues discussed above, include five-coordinate $\left[\mathrm{Cd}\left(\mathrm{Et}_{2} \mathrm{NCS}_{2}\right)_{2}\left(\mathrm{PEt}_{3}\right)\right] \quad(\mathrm{Cd}-\mathrm{P}=2.573(3) \AA) \quad[44], \quad\left[\mathrm{Cd}_{2}(\mu-\mathrm{O}-2,6-\right.$ $\left.\left.\mathrm{F}_{2} \mathrm{C}_{6} \mathrm{H}_{3}\right)_{2}\left(\mathrm{O}-2,6-\mathrm{F}_{2} \mathrm{C}_{6} \mathrm{H}_{3}\right)_{2}\left(\mathrm{PCy}_{3}\right)_{2}\right](\mathrm{Cd}-\mathrm{P}=2.543(1) \AA)[48]$ and $\left[\mathrm{Cd}\left(\mathrm{O}-2,6-\mathrm{F}_{2} \mathrm{C}_{6} \mathrm{H}_{3}\right)_{2}\left(\mathrm{PCy}_{3}\right)_{2}\right](\mathrm{Cd}-\mathrm{P}=2.648(2) \AA)[65]$.

A chain polymer with diphosphine bridges is present in $\left[\mathrm{CdI}_{2}\left\{\mathrm{Ph}_{2} \mathrm{P}\left(\mathrm{CH}_{2}\right)_{2} \mathrm{PPh}_{2}\right\}\right]_{n}$ [51]. The silicon-bridged diphosphine $\mathrm{Me}_{2} \mathrm{Si}\left(\mathrm{CH}_{2} \mathrm{PPh}_{2}\right)_{2}$ forms $1: 1$ adducts [CdX $\left.\left.2 \mathrm{Me}_{2} \mathrm{Si}\left(\mathrm{CH}_{2} \mathrm{PPh}_{2}\right)_{2}\right\}\right]$ $\left(\mathrm{X}=\mathrm{Cl}, \mathrm{Br}, \mathrm{I}, \mathrm{SCN}\right.$ or $\left.\mathrm{NO}_{3}\right)$ which were identified as distortedtetrahedral monomers by multinuclear NMR spectroscopy $\left({ }^{1} \mathrm{H}\right.$, $\left.{ }^{31} \mathrm{P}\left\{{ }^{1} \mathrm{H}\right\},{ }^{113} \mathrm{Cd}\right)$, and confirmed for the chloro-complex by an X-ray structure $(\mathrm{Cd}-\mathrm{P}=2.580(2), 2.553(2) \AA)$ [66]. The $\left[\mathrm{Cd}\left\{\mathrm{Me}_{2} \mathrm{Si}\left(\mathrm{CH}_{2} \mathrm{PPh}_{2}\right)_{2}\right\}_{2}\right]\left[\mathrm{ClO}_{4}\right]_{2}$ complex was also isolated. Ferrocenyl diphosphine complexes $\left[\mathrm{CdCl}_{2}\left\{\mathrm{Fe}\left(\mathrm{C}_{5} \mathrm{H}_{4} \mathrm{P}^{\mathrm{i}} \mathrm{Pr}_{2}\right)_{2}\right\}\right]$ and $\left[\mathrm{CdCl}_{2}\left\{\mathrm{Fe}\left(\mathrm{C}_{5} \mathrm{H}_{4} \mathrm{PCy}\right)_{2}\right\}\right]$, analogous to those of zinc, have been prepared [52,53], and the structure of $\left[\mathrm{CdCl}_{2}\left\{\mathrm{Fe}\left(\mathrm{C}_{5} \mathrm{H}_{4} \mathrm{PPh}_{2}\right)_{2}\right\}\right]$ determined $(\mathrm{Cd}-\mathrm{P}=2.453(2), 2.466(2) \AA)$ [67]. The pyridinephosphine $\left[\mathrm{Fe}\left(\mathrm{C}_{5} \mathrm{H}_{4} \mathrm{PPh}_{2}\right)\left(\mathrm{C}_{5} \mathrm{H}_{4}-2 \mathrm{py}\right)\right]$ forms $\mathrm{P}, \mathrm{N}$ chelate complexes with $\mathrm{CdBr}_{2}$ and $\mathrm{CdI}_{2}$, but whilst $\left[\mathrm{Fe}\left(\mathrm{C}_{5} \mathrm{H}_{4} \mathrm{PPh}_{2}\right)\left(\mathrm{C}_{5} \mathrm{H}_{4} \mathrm{CH}_{2}-2 \mathrm{py}\right)\right]$ also chelates to $\mathrm{CdI}_{2}$, it forms a ligand-bridged polymer with $\mathrm{CdBr}_{2}$ [58]. An unusual distorted pentagonal bipyramidal $\left(\mathrm{P}_{2} \mathrm{~S}_{2} \mathrm{NO}_{2}\right)$ geometry with axial phosphines $(\mathrm{Cd}-\mathrm{P}=2.590(1) \AA)$ is present in $[\mathrm{Cd}\{2,6$ $\left.\left(\mathrm{Ph}_{2} \mathrm{PCH}_{2} \mathrm{CH}_{2} \mathrm{SCH}_{2}\right)_{2} \mathrm{C}_{5} \mathrm{H}_{3} \mathrm{~N}\right\}\left(\mathrm{O}_{3} \mathrm{SCF}_{3}\right)_{2}$ ] [57].

\subsection{Mercury}

Although the basic types of mercury(II) phosphine and arsine complexes were established at much the same time as those of the cadmium analogues [29], mercury has remained an active area of research with significant amounts of new work reported in the last twenty years. The well-known $\left[\mathrm{HgX}_{2}\left(\mathrm{PR}_{3}\right)_{2}\right]$ type have the expected distorted tetrahedral geometries, and have been much studied by X-ray crystallography and ${ }^{31} \mathrm{P}\left\{{ }^{1} \mathrm{H}\right\}$ and ${ }^{199} \mathrm{Hg}$ NMR $(I=1 / 2,17 \%, \mathrm{Rc}=5.7)$ spectroscopy, with the Hg-P bond lengths, $<\mathrm{P}-\mathrm{Hg}-\mathrm{P}$ angles and ${ }^{1} \mathrm{~J}\left({ }^{31} \mathrm{P}-{ }^{199} \mathrm{Hg}\right)$ coupling constants all showing a significant dependence upon the halide and the phosphine present. Solution NMR studies have also revealed that a mixture of complexes is often present in solution, and more recently, solution and CP MAS NMR studies have been used to correlate the solid and solution structures. There is a large amount of ${ }^{31} \mathrm{P}\left\{{ }^{1} \mathrm{H}\right\}$ and ${ }^{199} \mathrm{Hg}$ NMR data available $[20,62,69,70]$. The ${ }^{199} \mathrm{Hg}$ chemical shifts cover a wide range and are sensitive to solvent and temperature, as well as to chemical exchange effects, hence comparing data between different studies obtained under different experimental conditions needs to be done with some care. Table 3 illustrates the usually rather systematic trends observed on changing the chemical speciation.

Reported X-ray structures are listed in Table 4. Recent interest has focused on the 1:1 complexes for which isomers of the $\left[\mathrm{Hg}_{2} \mathrm{X}_{2}(\mu-\mathrm{X})_{2}\left(\mathrm{PR}_{3}\right)_{2}\right]$ type have been found, and on some other stoichiometries, as well as the subtle relationships between $1: 1$ and 2:1 complexes, especially with phosphines with large cone angles. The large amount of structural data available has also shown that intermolecular effects such as long halide bridges or $\pi$-stacking play a role in determining the structures adopted $[71,72]$. The complexes of $\mathrm{P}^{n} \mathrm{Pr}_{3}$ illustrate the complexities observed [72,73]. The 
Table 4

Recent X-ray structural data on mercury(II) complexes.

\begin{tabular}{|c|c|c|c|}
\hline Complex & structure & $\mathrm{Hg}-\mathrm{P}(\AA)$ & Refs. \\
\hline$\left[\mathrm{HgCl}_{2}\left(\mathrm{PPh}_{3}\right)_{2}\right]$ & Tetrahedral & $2.503(6), 2.532(4)$ & [100] \\
\hline$\left[\mathrm{HgBr}_{2}\left(\mathrm{PPh}_{3}\right)_{2}\right]$ & Tetrahedral & $2.550(4), 2.491(5)$ & [101] \\
\hline$\left[\mathrm{HgCl}\left(\mathrm{PPh}_{3}\right)(\mu-\mathrm{Cl})_{2} \mathrm{HgCl}\left(\mathrm{PPh}_{3}\right)\right]$ & Sym. dimer & $2.3991(8)$ & [71] \\
\hline$\left[\mathrm{HgBr}\left(\mathrm{PPh}_{3}\right)(\mu-\mathrm{Br})_{2} \mathrm{HgBr}\left(\mathrm{PPh}_{3}\right)\right]$ & Sym. dimer & $2.40(2), 2.44(2)$ & [69] \\
\hline$\left[\mathrm{HgI}\left(\mathrm{PPh}_{3}\right)(\mu-\mathrm{I})_{2} \mathrm{HgI}\left(\mathrm{PPh}_{3}\right)\right]$ & Sym. dimer & $2.4724(10)$ & [71] \\
\hline$\left[\mathrm{HgCl}\left(\mathrm{PCy}_{3}\right)(\mu-\mathrm{Cl})_{2} \mathrm{HgCl}\left(\mathrm{PC}_{3}\right)\right]$ & Sym. dimer & $2.410(1)$ & [102] \\
\hline$\left[\mathrm{HgCl}\left(\mathrm{P}^{n} \mathrm{Pr}_{3}\right)(\mu-\mathrm{Cl})_{2} \mathrm{HgCl}\left(\mathrm{P}^{n} \mathrm{Pr}_{3}\right)\right]$ & Sym. dimer & $2.358(4)$ & [72] \\
\hline$\left[\mathrm{HgBr}\left(\mathrm{P}^{n} \mathrm{Pr}_{3}\right)(\mu-\mathrm{Br})_{2} \mathrm{HgBr}\left(\mathrm{P}^{n} \operatorname{Pr}_{3}\right)\right]$ & Sym. dimer & $2.408(4)$ & [72] \\
\hline$\left[\mathrm{HgI}_{2}\left(\mathrm{P}^{n} \mathrm{Pr}_{3}\right)\right]_{\mathrm{n}}$ & Polymer & $2.406(7)$ & [72] \\
\hline$\left[\mathrm{HgI}_{2}(\mu-\mathrm{I})_{2} \mathrm{Hg}\left(\mathrm{P}^{n} \mathrm{Pr}_{3}\right)_{2}\right]$ & Asym. dimer & $2.457(14), 2.421(15)$ & {$[72,73]$} \\
\hline$\left[\mathrm{Hg}\left(\mathrm{P}^{n} \mathrm{Pr}_{3}\right)_{2}(\mu-\mathrm{I})_{2} \mathrm{CdI}_{2}\right]$ & Asym. dimer & $2.424(4), 2.410(4)$ & [68] \\
\hline$\left[\mathrm{HgI}_{2}(\mu-\mathrm{I})_{2} \mathrm{Hg}\left(\mathrm{P}^{n} \mathrm{Bu}_{3}\right)_{2}\right]$ & Asym. dimer & $2.391(22)-2.410(17)$ & [69] \\
\hline$\left[\left(\mathrm{HgI}_{2}\right)_{3}\left(\mathrm{PMe}_{3}\right)_{2}\right]$ & See text & $2.413(6)$ & [74] \\
\hline$\left[\mathrm{HgI}_{2}\left\{\mathrm{Ph}_{2} \mathrm{P}\left(\mathrm{CH}_{2}\right)_{2} \mathrm{PPh}_{2}\right\}\right]_{n}$ & Chain polymer & $2.5037(14), 2.5064(13)$ & [51] \\
\hline$\left[\mathrm{HgBr}_{2}(1 \text {-Ph-dibenzophosphole })_{2}\right]$ & Tetrahedral & $2.490(3), 2.513(3)$ & [69] \\
\hline$\left.\left[\mathrm{Hg}_{2} \mathrm{I}_{4(} \text { 1-Ph-Me } \mathrm{Me}_{2} \text { dibenzophosphole }\right)_{2}\right]$ & Sym. dimer & $2.437(7), 2.470(7)$ & [69] \\
\hline$\left[\mathrm{HgBr}_{2}\left\{\mathrm{PPh}(2-\mathrm{py})_{2}\right\}_{2}\right]$ & Tetrahedral $\left(\mathrm{P}_{2} \mathrm{Br}_{2}\right)$ & $2.512(2), 2.494(2)$ & [75] \\
\hline$\left[\mathrm{Hg}\left(\mathrm{PPh}_{3}\right)_{2}(\mu-\mathrm{SCN}) \mathrm{Cd}(\mathrm{SCN})_{3}\right]$ & Polymer & $2.501(1), 2.483(1)$ & [103] \\
\hline$\left[\mathrm{Hg}\left\{\mathrm{OP}(\mathrm{O}) \mathrm{CF}_{3}(\mathrm{OH})\right\}_{2}\left(\mathrm{PMe}_{3}\right)_{3}\right]$ & Polymer & $2.449(2)-2.481(2)$ & [104] \\
\hline$\left[\mathrm{Hg}\left\{\mathrm{P}\left(2,4,6-(\mathrm{MeO})_{3} \mathrm{C}_{6} \mathrm{H}_{2}\right)_{3}\right\}_{2}\right]\left[\mathrm{Hg}_{2} \mathrm{Cl}_{6}\right]$ & 2-Coordinate & $2.389(5)$ & [76] \\
\hline$\left[\mathrm{Hg}_{2} \mathrm{Cl}_{4}\left\{\mathrm{P}\left(2,6-(\mathrm{MeO})_{2} \mathrm{C}_{6} \mathrm{H}_{3}\right)_{3}\right\}_{2}\right]$ & Sym. dimer & $2.436(3)$ & [77] \\
\hline$\left[\mathrm{H}_{\mathrm{g} 2} \mathrm{Br}_{4}\left\{\mathrm{P}\left(2,6-(\mathrm{MeO})_{2} \mathrm{C}_{6} \mathrm{H}_{3}\right)_{3}\right\}_{2}\right]$ & Sym. dimer & $2.4490(12)$ & [77] \\
\hline$\left[\mathrm{HgI}_{2}\left\{\mathrm{P}\left(2,6-(\mathrm{MeO})_{2} \mathrm{C}_{6} \mathrm{H}_{3}\right)_{3}\right\}\right]$ & 3-Coord. monomer & $2.4656(16)$ & [77] \\
\hline$\left[\mathrm{HgBr}_{2}\left\{\mathrm{P}\left(2,4,6-(\mathrm{MeO})_{3} \mathrm{C}_{6} \mathrm{H}_{3}\right)_{3}\right\}\right]$ & 3-Coord. monomer & $2.420) 4)$ & [78] \\
\hline$\left[\mathrm{HgI}_{2}\left\{\mathrm{P}\left(2,4,6-(\mathrm{MeO})_{3} \mathrm{C}_{6} \mathrm{H}_{3}\right)_{3}\right\}\right]$ & 3-Coord. monomer & $2.437(2)$ & [78] \\
\hline$\left[\mathrm{Hg}_{2} \mathrm{I}_{4}\left\{\mathrm{P}\left(2,4,6-(\mathrm{MeO})_{3} \mathrm{C}_{6} \mathrm{H}_{3}\right)_{3}\right\}\right]$ & See text & $2.408(2)$ & [78] \\
\hline$\left[\mathrm{Hg}\left(\mathrm{PBz}_{3}\right)_{2}\right]\left[\mathrm{BF}_{4}\right]_{2}$ & Linear & $2.4032(5)$ & [79] \\
\hline$\left[\mathrm{Hg}\left(\mathrm{PBz}_{3}\right)_{2}\left(\mathrm{NO}_{3}\right)_{2}\right]$ & $4+2$ Coordinate & $2.429(1)-2.435(1)$ & [79] \\
\hline$\left[\mathrm{Hg}\left(\mathrm{PBz}_{3}\right)_{2} \mathrm{Cl}\left(\mathrm{NO}_{3}\right)\right]$ & Chain polymer & $2.371(3)$ & [79] \\
\hline$\left[\mathrm{Hg}\left(\mathrm{PBz}_{3}\right)_{2} \mathrm{Br}\left(\mathrm{NO}_{3}\right)\right]$ & Chain polymer & $2.373(2)$ & [79] \\
\hline$\left[\mathrm{Hg}\left(\mathrm{PBz}_{3}\right)_{2} \mathrm{I}\left(\mathrm{NO}_{3}\right)\right]$ & Chain polymer & $2.392(1)$ & [79] \\
\hline$\left[\mathrm{Hg}\left(\mathrm{PBz}_{3}\right)_{2}(\mathrm{SCN})\left(\mathrm{NO}_{3}\right)\right]$ & $4+2$ Coordinate dimer & $2.396(1)$ & [79] \\
\hline$\left[\mathrm{Hg}\left(\mathrm{PPh}_{3}\right) \mathrm{Cl}\left(\mathrm{NO}_{3}\right)\right]$ & Polymer & $2.372(1)$ & [80] \\
\hline$\left[\mathrm{Hg}\left(\mathrm{PPh}_{3}\right) \mathrm{Br}\left(\mathrm{NO}_{3}\right)\right]$ & Polymer & $2.388(1)$ & [80] \\
\hline$\left[\mathrm{Hg}\left(\mathrm{PPh}_{3}\right) \mathrm{I}\left(\mathrm{NO}_{3}\right)\right]$ & Polymer & $2.402(2)$ & [80] \\
\hline$\left[\mathrm{Hg}\left\{\mathrm{Ph}_{2} \mathrm{P}\left(\mathrm{CH}_{2}\right)_{n} \mathrm{PPh}_{2}\right)_{2}\right]\left[\mathrm{O}_{3} \mathrm{SCF}_{3}\right]_{2}$ & Tetrahedral & $2.512(4), 2.613(4)$ & [82] \\
\hline$\left.\left[\mathrm{Hg}\left\{\mathrm{P}\left(\mathrm{CF}_{3}\right)_{2}\right\}_{2}\left\{\mathrm{Ph}_{2} \mathrm{P}\left(\mathrm{CH}_{2}\right)_{2} \mathrm{PPh}_{2}\right)\right\}\right]$ & Tetrahedral & $2.71(4), 2.571(4)^{\varphi}$ & [83] \\
\hline$\left[\mathrm{HgI}_{2}\left\{\mathrm{Ph}_{2} \mathrm{PCH}_{2} \mathrm{SiMe}_{2} \mathrm{CH}_{2} \mathrm{PPh}_{2}\right\}\right]$ & Tetrahedral & $2.515(2), 2.511(2)$ & [105] \\
\hline$\left[\mathrm{HgCl}_{2}\left\{\mathrm{Fe}\left(\mathrm{C}_{5} \mathrm{H}_{4} \mathrm{PPh}_{2}\right)_{2}\right\}\right]$ & Tetrahedral & $2.5102(17), 2.5162(16)$ & [84] \\
\hline$\left[\mathrm{HgI}_{2}\left\{\mathrm{Fe}\left(\mathrm{C}_{5} \mathrm{H}_{4} \mathrm{PPh}_{2}\right)_{2}\right\}\right]$ & Tetrahedral & $2.5585(15), 2.5718(14)$ & [85] \\
\hline$\left[\mathrm{HgBr}_{2}\left\{\mathrm{Fe}\left(\mathrm{C} \mathrm{H}_{4} \mathrm{PPh}_{2}\right)\left(\mathrm{C}_{5} \mathrm{H}_{4} \mathrm{CO}_{2} \mathrm{H}\right)\right\}_{2}\right]$ & Tetrahedral & $2.529(2)$ & [86] \\
\hline$\left[\mathrm{H}_{\mathrm{g} 2} \mathrm{Br}_{4}\left\{\mathrm{Fe}\left(\mathrm{C}_{5} \mathrm{H}_{4} \mathrm{PPh}_{2}\right)\left(\mathrm{C}_{5} \mathrm{H}_{4} \mathrm{CO}_{2} \mathrm{H}\right)\right\}_{2}\right]$ & Sym. dimer & $2.425(1)$ & [86] \\
\hline$\left[\mathrm{HgBr}_{2}\left\{\mathrm{Fe}\left(\mathrm{C}_{5} \mathrm{H}_{4} \mathrm{PPh}_{2}\right)\left(\mathrm{C}_{5} \mathrm{H}_{4}-2 \mathrm{py}\right)\right\}\right]$ & Chelate $\mathrm{PNX}_{2}$ & $2.4462(9)$ & [58] \\
\hline$\left[\mathrm{HgCl}_{2}\left\{\mathrm{Fe}\left(\mathrm{C}_{5} \mathrm{H}_{4} \mathrm{PPh}_{2}\right)\left(\mathrm{C}_{5} \mathrm{H}_{4}-2 \mathrm{py}\right)\right\}_{2}\right]$ & Tetrahedral $\mathrm{P}_{2} \mathrm{X}_{2}$ & $2.495(6)$ & [58] \\
\hline$\left[\mathrm{HgBr}_{2}\left\{\mathrm{Fe}\left(\mathrm{C}_{5} \mathrm{H}_{4} \mathrm{PPh}_{2}\right)\left(\mathrm{C}_{5} \mathrm{H}_{4}-2 \mathrm{py}\right)\right\}_{2}\right]$ & Tetrahedral $\mathrm{P}_{2} \mathrm{X}_{2}$ & $2.503(1), 2.497(1)$ & [58] \\
\hline$\left[\mathrm{HgBr}_{2}\left\{\mathrm{Fe}\left(\mathrm{C}_{5} \mathrm{H}_{4} \mathrm{PPh}_{2}\right)\left(\mathrm{C}_{5} \mathrm{H}_{4} \mathrm{CH}_{2}-2 \mathrm{py}\right)\right\}\right]$ & Chelate $\mathrm{PNX}_{2}$ & $2.443(2)$ & [58] \\
\hline$\left[\mathrm{HgBr}_{2}\left\{\mathrm{Fe}\left(\mathrm{C}_{5} \mathrm{H}_{4} \mathrm{PPh}_{2}\right)\left(\mathrm{C}_{5} \mathrm{H}_{4} \mathrm{CH}_{2}-2 \mathrm{py}\right)\right\}_{2}\right]$ & Tetrahedral $\mathrm{P}_{2} \mathrm{X}_{2}$ & $2.510(2), 2.512(2)$ & [58] \\
\hline$\left[\mathrm{Hg}_{2} \mathrm{Br}_{4}\left\{\mathrm{Fe}\left(\mathrm{C}_{5} \mathrm{H}_{4} \mathrm{PPh}_{2}\right)\left(\mathrm{C}_{5} \mathrm{H}_{4} \mathrm{CH}_{2}-2 \mathrm{py}\right)\right\}\right]_{n}$ & Polymer $\mathrm{PX}_{3}$ & $2.510(2), 2.512(2)$ & [58] \\
\hline$\left[\mathrm{HgBr}_{2}\left\{\mathrm{Fe}\left(\mathrm{C}_{5} \mathrm{H}_{4} \mathrm{PPh}_{2}\right)\left(\mathrm{C}_{5} \mathrm{H}_{4}-3 \mathrm{py}\right)\right\}\right]_{n}$ & Polymer $\mathrm{PNX}_{2}$ & $2.442(1)$ & [58] \\
\hline$\left[\mathrm{HgBr}_{2}\left(\mathrm{Ph}_{2} \mathrm{PCH}_{2} \mathrm{CH}_{2} \mathrm{SEt}\right)\right]$ & $3+1$ Coordinate & $2.415(1)$ & [106] \\
\hline$\left[\mathrm{Hg}_{3} \mathrm{Cl}_{2}(\mu-\mathrm{Cl})_{2}\left\{\mathrm{PhP}\left(\mathrm{CH}_{2} \mathrm{PPh}_{2}\right)_{2}\right\}_{2}\right]^{2+}$ & See text & $2.442(9)-2.461(8)$ & [87] \\
\hline$\left[\mathrm{Hg}_{2} \mathrm{Cl}_{4}\left\{\mathrm{C}_{4} \mathrm{H}_{4}\left(\mathrm{PPh}_{2}\right)_{4}\right\}\right]$ & See text & $2.470(2)-2.629(2)$ & [88] \\
\hline$\left[\mathrm{Hg}_{2} \mathrm{Br}_{4}\left\{\mathrm{C}_{4} \mathrm{H}_{4}\left(\mathrm{PPh}_{2}\right)_{4}\right\}\right]$ & See text & $2.532(2)-2.597(1)$ & [88] \\
\hline$\left[\mathrm{Hg}_{2}(\mathrm{CN})_{4}\left\{\mathrm{C}_{4} \mathrm{H}_{4}\left(\mathrm{PPh}_{2}\right)_{4}\right\}\right]$ & See text & $2.517(1), 2.543(1)$ & [88] \\
\hline$\left[\mathrm{Hg}_{2}\left(\mathrm{NO}_{3}\right)_{2}\left\{\mathrm{C}_{4} \mathrm{H}_{4}\left(\mathrm{PPh}_{2}\right)_{4}\right\}\left(\kappa^{1}-\mathrm{Ph}_{2} \mathrm{PCH}_{2} \mathrm{PPh}_{2}\right)_{2}\right]\left[\mathrm{AsF}_{6}\right]_{2}$ & See text & $2.409(3)-2.555(3)$ & [88] \\
\hline$\left[\mathrm{HgI}\left\{\mathrm{N}\left(\mathrm{CH}_{2} \mathrm{CH}_{2} \mathrm{PPh}_{2}\right)_{3}\right\}\right] \mathrm{I}$ & Tetrahedral $\left(\mathrm{P}_{3} \mathrm{I}\right)$ & $2.521(5)-2.555(7)$ & [89] \\
\hline$\left[\mathrm{Hg}(\mathrm{Me})\left\{\mathrm{N}\left(\mathrm{CH}_{2} \mathrm{CH}_{2} \mathrm{PPh}_{2}\right)_{3}\right\}\right]\left[\mathrm{CF}_{3} \mathrm{SO}_{3}\right]$ & Tetrahedral & $2.600(8)-2.808(7)$ & {$[90,92]$} \\
\hline$\left[\mathrm{Hg}\left(\mathrm{C}_{6} \mathrm{~F}_{5}\right)\left\{\mathrm{N}\left(\mathrm{CH}_{2} \mathrm{CH}_{2} \mathrm{PPh}_{2}\right)_{3}\right\}\right]\left[\mathrm{CF}_{3} \mathrm{SO}_{3}\right]$ & Tetrahedral & $2.584(13)-2.612(10)$ & [91] \\
\hline$\left[\mathrm{Hg}\left(S-p-\mathrm{C}_{6} \mathrm{H}_{4} \mathrm{Me}\right)\left\{N\left(\mathrm{CH}_{2} \mathrm{CH}_{2} \mathrm{PPh}_{2}\right)_{3}\right\}\right]\left[\mathrm{CF}_{3} \mathrm{SO}_{3}\right]$ & Tetrahedral & $2.539(7)-2.633(8)$ & [92] \\
\hline$\left[\mathrm{Hg}(\mathrm{Me})\left\{\mathrm{P}\left(\mathrm{CH}_{2} \mathrm{CH}_{2} \mathrm{PPh}_{2}\right)_{3}\right\}\right]\left[\mathrm{BF}_{4}\right]$ & $2+2$ & $2.446(3), 2.879(3), 3.078(3)$ & [94] \\
\hline
\end{tabular}

$\left[\mathrm{HgX}\left(\mathrm{P}^{n} \mathrm{Pr}_{3}\right)(\mu-\mathrm{X})_{2} \mathrm{HgX}\left(\mathrm{P}^{n} \mathrm{Pr}_{3}\right)\right](\mathrm{X}=\mathrm{Cl}$ or $\mathrm{Br})$ have the typical symmetrical dimer structure, but the iodide has two forms; one is also dimeric but with both phosphines coordinated to one mercury (Fig. 8) whereas the second form has a trigonal planar $\left(\mathrm{I}_{2} \mathrm{P}\right)$ core, with very long $\mathrm{Hg}-\mathrm{I}$ contacts (3.570(5), 3.627(5) $\AA$ ) to neighbouring molecules, creating a trigonal bipyramidal chain (Fig. 9) [72]. Curiously, the ${ }^{31} \mathrm{P}\left\{{ }^{1} \mathrm{H}\right\}$ NMR spectrum of $\left[\mathrm{HgI}_{2}(\mu-\mathrm{I})_{2} \mathrm{Hg}\left(\mathrm{P}^{n} \mathrm{Pr}_{3}\right)_{2}\right]$ suggests the symmetrical isomer is the major form in solution. Unsymmetrical dimers of this type have been previously observed as minor species in solution for $\mathrm{P}^{n} \mathrm{Bu}_{3}$ complexes [70], and subsequently $\left[\mathrm{HgI}_{2}(\mu-\mathrm{I})_{2} \mathrm{Hg}\left(\mathrm{P}^{n} \mathrm{Bu}_{3}\right)_{2}\right]$ was structurally characterised [69]. Some Cd/Hg heterobimetallics $\left[\mathrm{CdI}_{2}(\mu-\mathrm{I})_{2} \mathrm{Hg}\left(\mathrm{PR}_{3}\right)_{2}\right.$ ] have been obtained and $\left[\mathrm{CdI}_{2}(\mu-\mathrm{I})_{2} \mathrm{Hg}\left(\mathrm{P}^{n} \mathrm{Pr}_{3}\right)_{2}\right]$ has been structurally characterised [68]. The structure of the 2:3 complex $\left[\left(\mathrm{HgI}_{2}\right)_{3}\left(\mathrm{PMe}_{3}\right)_{2}\right]$ reveals it is best described as containing linear $\left[\mathrm{Hg}\left(\mathrm{PMe}_{3}\right)_{2}\right]^{2+}$ cations weakly interacting with four $\left[\mathrm{HgI}_{3}\right]^{-}$ions to give a chain structure, further cross-linked by long $\mathrm{Hg}-\mathrm{I}-\mathrm{Hg}$ contacts [74].

Pyridylphosphines, such as $\mathrm{P}\left(2-\mathrm{C}_{5} \mathrm{H}_{4} \mathrm{~N}\right)_{3}$ and $\mathrm{P}\left(2-\mathrm{C}_{5} \mathrm{H}_{4} \mathrm{~N}\right)_{2} \mathrm{Ph}$ form $2: 1$ adducts $\left[\mathrm{HgX}_{2} \mathrm{~L}_{2}\right](\mathrm{X}=\mathrm{Cl}$ or $\mathrm{Br})$, in which the ligand bonds to $\mathrm{Hg}$ only via the phosphorus atoms, although the pyridyl rings are involved in $\pi$-stacking interactions in the crystals [75].

The chemistry of mercury(II) halides with very bulky phosphines such as tris(2,6-dimethoxyphenyl)phosphine (DMPP) or 


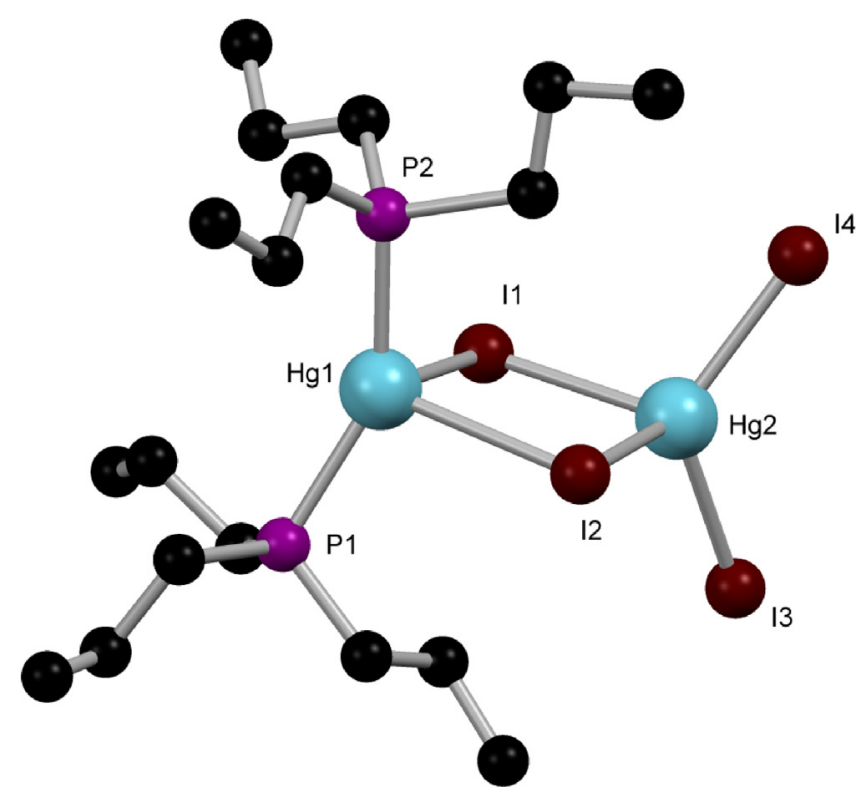

Fig. 8. View of the structure of $\left[\mathrm{HgI}_{2}(\mu-\mathrm{I})_{2} \mathrm{Hg}\left(\mathrm{P}^{n} \mathrm{Pr}_{3}\right)_{2}\right]$ redrawn from Ref. [72].

tris(2,4,6-trimethoxyphenyl)phosphine (TMPP) which have cone angles $\sim 184^{\circ}$ is very different to that with smaller phosphines [76-78]. Acetonitrile solutions of $\mathrm{HgX}_{2}$ and TMPP contain both $[\mathrm{HgX}(\mathrm{TMPP})]^{+}$and $\left[\mathrm{Hg}(\mathrm{TMPP})_{2}\right]^{2+}$ ions, and crystallisation results in solids containing these cations and $\left[\mathrm{Hg}_{2} \mathrm{X}_{6}\right]^{2-}$. The structure of $\left[\mathrm{Hg}(\mathrm{TMPP})_{2}\right]\left[\mathrm{Hg}_{2} \mathrm{Cl}_{6}\right]$ reveals a slightly bent $\mathrm{P}-\mathrm{Hg}-\mathrm{P}$ unit $\left(166.5^{\circ}\right)$ with contacts between the $\mathrm{Hg}$ and three methoxy oxygens $\sim 2.7-3.0 \AA$. From a 1:1 Hg:TMPP ratio in ethanol the products were trigonal planar monomers $\left[\mathrm{HgX}_{2}(\mathrm{TMPP})\right](\mathrm{X}=\mathrm{Br}$ or I), whilst a second product with a 2:1 Hg:TMPP composition is the oligomer [(TMPP) $\left.\mathrm{HgI}(\mu-\mathrm{I}) \mathrm{HgI}(\mu-\mathrm{I})_{2} \mathrm{HgI}(\mu-\mathrm{I}) \mathrm{HgI}(\mathrm{TMPP})\right]$. It was also possible to isolate $\left.\left[\mathrm{HgX}_{2} \text { (TMPP) }\right)_{2}\right]$ complexes, presumed to be tetrahedral monomers, although these lose one TMPP molecule very easily in solution. The rather weaker $\sigma$-donor phosphine DMPP forms only $1: 1$ complexes, which are halide bridged dimers $[\operatorname{HgX}(\mathrm{DMPP})(\mu$ $\left.\mathrm{X})_{2} \mathrm{HgX}(\mathrm{DMPP})\right]$ for $\mathrm{X}=\mathrm{Cl}$ or $\mathrm{Br}$, but the iodide is a trigonal planar monomer. Tribenzylphosphine also has a large cone angle $\left(165^{\circ}\right)$ resulting in different chemistry compared to small phosphines. Thus it gives $\left[\mathrm{Hg}\left(\mathrm{PBz}_{3}\right)_{2}\right]\left[\mathrm{BF}_{4}\right]_{2}$ (with a linear 2-coordinate $\mathrm{P}-\mathrm{Hg}-\mathrm{P}$ moiety) (Fig. 10), $\left[\mathrm{Hg}\left(\mathrm{PBz}_{3}\right)_{2}\left(\mathrm{NO}_{3}\right)_{2}\right]$ (a monomer which has a distorted $\mathrm{P}_{2} \mathrm{O}_{2}$ core with longer contacts to two more nitrate $\mathrm{O}$ giving $4+2$ coordination $),\left[\mathrm{Hg}\left(\mathrm{PBz}_{3}\right)_{2} \mathrm{X}\left(\mathrm{NO}_{3}\right)\right](\mathrm{X}=\mathrm{Cl}, \mathrm{Br}$

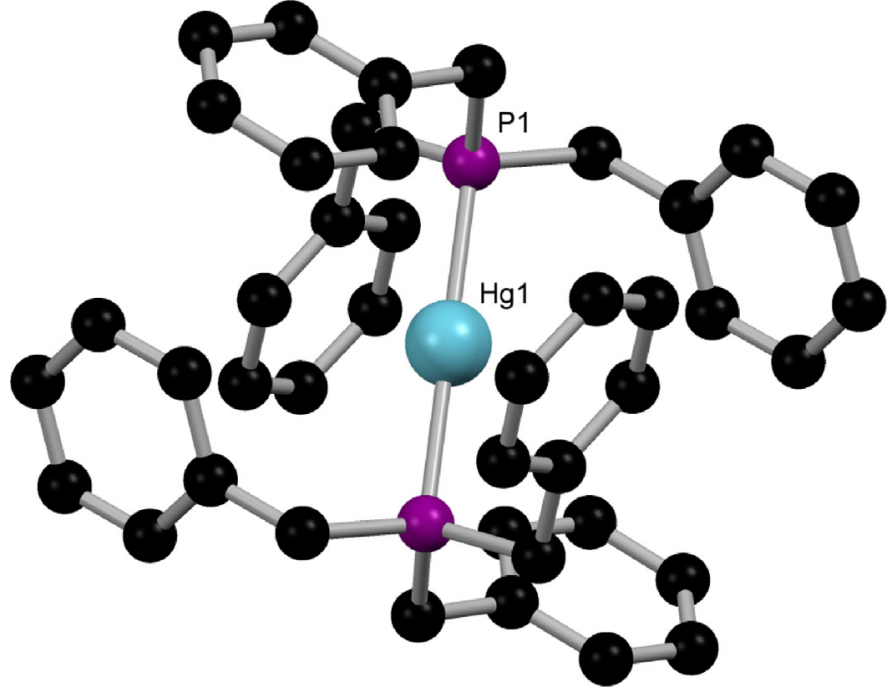

Fig. 10. View of the structure of the cation $\left[\mathrm{Hg}\left(\mathrm{PBz}_{3}\right)_{2}\right]^{2+}$ redrawn from Ref. [79].

or I: polymers with $4+2$ coordination and nitrato-bridges), and $\left[\mathrm{Hg}\left(\mathrm{PBZ}_{3}\right)_{2}(\mathrm{SCN})\left(\mathrm{NO}_{3}\right)\right]$ (a discrete dimer with $\mathrm{SCN}$ bridges and with $4+2$ coordination) [79].

Complexes of type $\left[\mathrm{Hg}\left(\mathrm{PPh}_{3}\right) \mathrm{X}\left(\mathrm{NO}_{3}\right)\right](\mathrm{X}=\mathrm{Cl}, \mathrm{Br}$ or I) contain slightly bent $\mathrm{X}-\mathrm{Hg}-\mathrm{P}$ units linked by nitrate groups into polymeric chains [80]. Although they are genuine mixed anion salts in the solid state, shown both by the crystallographic results and CP MAS NMR studies, in solution ${ }^{31} \mathrm{P}\left\{{ }^{1} \mathrm{H}\right\}$ NMR spectroscopy shows they partially rearrange into $\left[\mathrm{Hg}\left(\mathrm{PPh}_{3}\right)_{2}\left(\mathrm{NO}_{3}\right)_{2}\right]$ and $\mathrm{HgX}$.

No new studies of mercury(II) arsines have appeared, but $\left[\mathrm{HgX}_{2}\left\{\mathrm{Sb}\left(2,4,6-\mathrm{Me}_{3} \mathrm{C}_{6} \mathrm{H}_{2}\right)_{3}\right\}\right](\mathrm{X}=\mathrm{Cl}, \mathrm{Br}, \mathrm{I}$ or $\mathrm{SCN})$ have been described, although structural data are lacking [81].

The $\left[\mathrm{HgI}_{2}\left\{\mathrm{Ph}_{2} \mathrm{P}\left(\mathrm{CH}_{2}\right)_{2} \mathrm{PPh}_{2}\right\}\right]_{n}$ is a diphosphine-bridged chain polymer [51]. Binuclear complexes [MeHg$(\mu$ $\left.\mathrm{Ph}_{2} \mathrm{P}\left(\mathrm{CH}_{2}\right)_{n} \mathrm{PPh}_{2}\right) \mathrm{HgMe}_{[}\left[\mathrm{O}_{3} \mathrm{SCF}_{3}\right]_{2} \quad(n=1-3)$ form on mixing $[\mathrm{MeHg}(\mathrm{dmso})]\left[\mathrm{O}_{3} \mathrm{SCF}_{3}\right]$ and the diphosphine in a 2:1 ratio in $\mathrm{CH}_{2} \mathrm{Cl}_{2}$ [82]. An excess of diphosphine causes dismutation into $\mathrm{HgMe}_{2}$ and $\left[\mathrm{Hg}\left\{\mathrm{Ph}_{2} \mathrm{P}\left(\mathrm{CH}_{2}\right)_{n} \mathrm{PPh}_{2}\right)_{2}\right]\left[\mathrm{O}_{3} \mathrm{SCF}_{3}\right]_{2}$. The complex $\left[\mathrm{Hg}\left\{\mathrm{P}\left(\mathrm{CF}_{3}\right)_{2}\right\}_{2}\left\{\mathrm{Ph}_{2} \mathrm{P}\left(\mathrm{CH}_{2}\right)_{2} \mathrm{PPh}_{2}\right\}\right]$ is formed by treating the corresponding dicyano-mercury complex with $\mathrm{PH}\left(\mathrm{CF}_{3}\right)_{2}[83]$. The ligand $\mathrm{Ph}_{2} \mathrm{PCH}_{2} \mathrm{SiMe}_{2} \mathrm{CH}_{2} \mathrm{PPh}_{2}$ forms tetrahedral chelate complexes with $\mathrm{HgX}_{2}(\mathrm{X}=\mathrm{Cl}, \mathrm{Br}, \mathrm{I}, \mathrm{SCN})$ [105].

A range of ferrocenyl diphosphine complexes of $\mathrm{Hg}(\mathrm{II})$ has been reported which all appear to contain simple

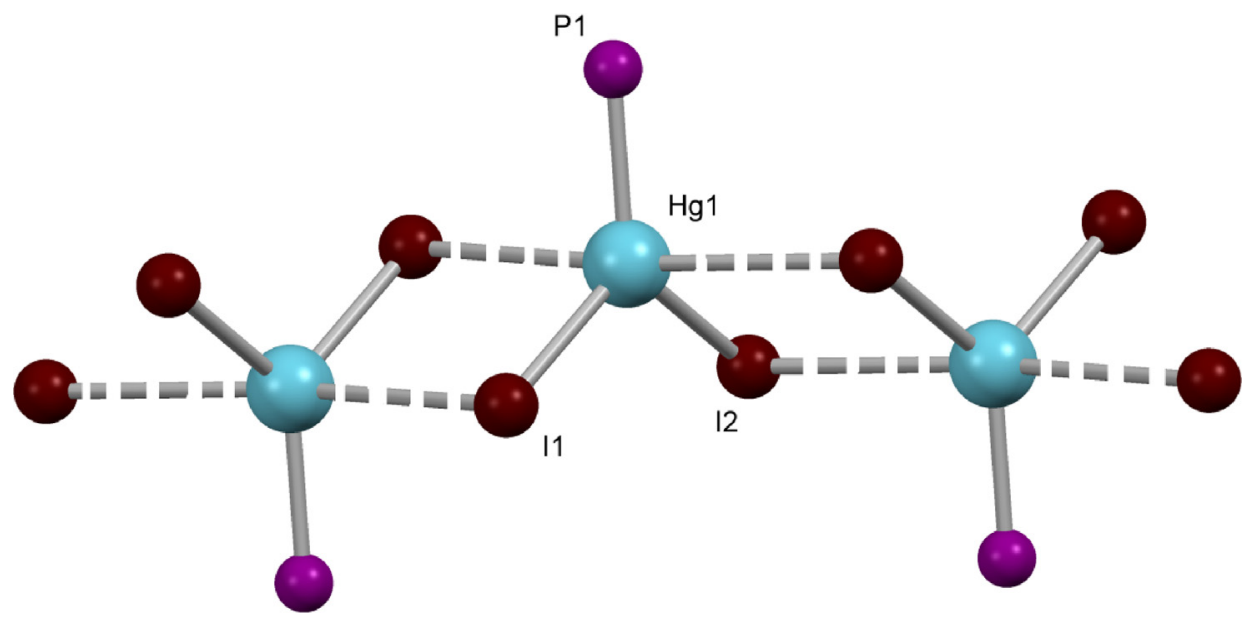

Fig. 9. View of the chain structure of $\left[\mathrm{HgI}_{2}\left(\mathrm{P}^{n} \mathrm{Pr}_{3}\right)\right]$ redrawn from Ref. [72]. Propyl groups have been omitted for clarity. 


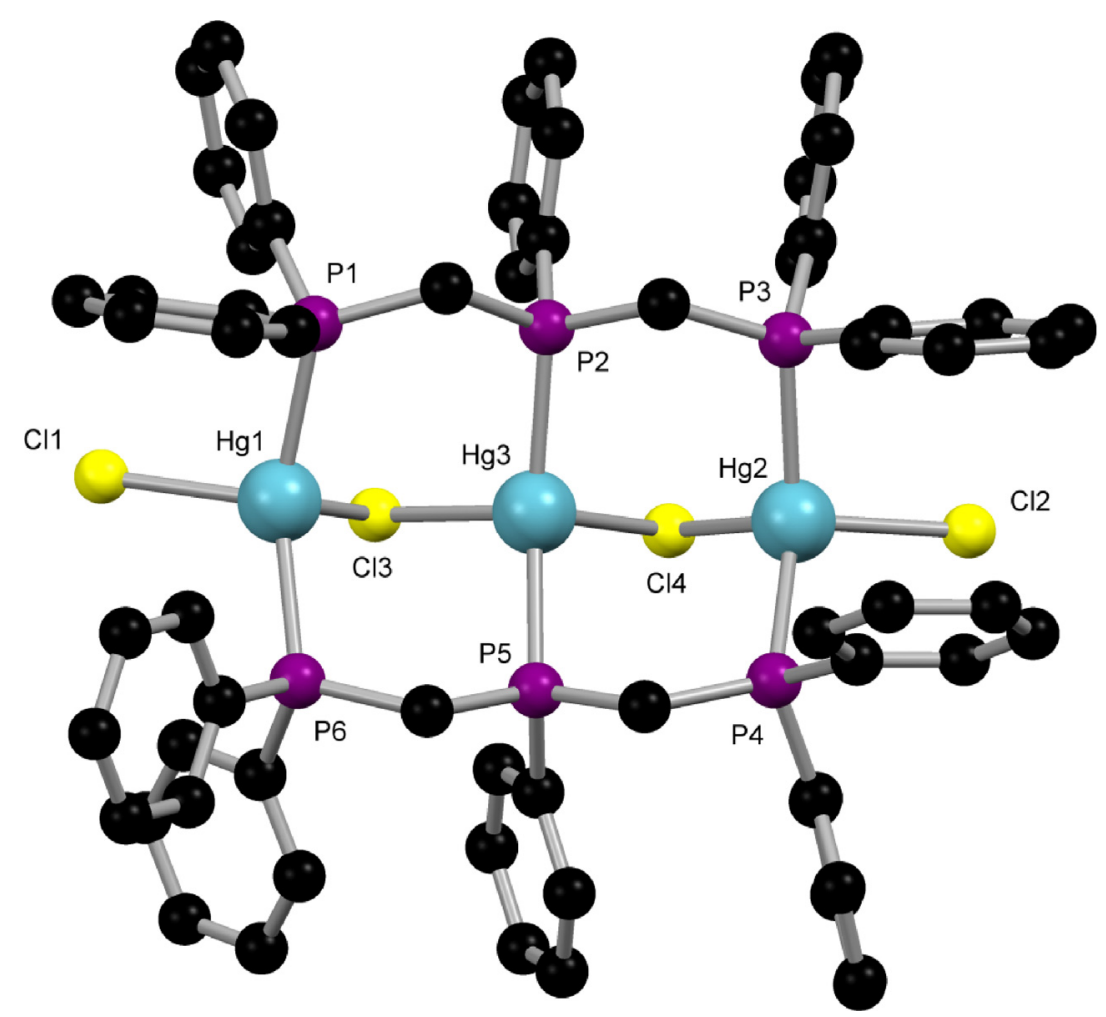

Fig. 11. View of the structure of the cation $\left[\mathrm{Hg}_{3} \mathrm{Cl}_{2}(\mu-\mathrm{Cl})_{2}\left\{\mathrm{PhP}\left(\mathrm{CH}_{2} \mathrm{PPh}_{2}\right)_{2}\right\}_{2}\right]^{2+}$ redrawn from Ref. [87].

tetrahedral monomers, including $\left[\mathrm{HgCl}_{2}\left\{\mathrm{Fe}\left(\mathrm{C}_{5} \mathrm{H}_{4} \mathrm{P}^{i} \mathrm{Pr}_{2}\right)_{2}\right\}\right]$ [52], [HgCl$\left.{ }_{2}\left\{\mathrm{Fe}\left(\mathrm{C}_{5} \mathrm{H}_{4} \mathrm{P}^{t} \mathrm{Bu}_{2}\right)_{2}\right\}\right], \quad\left[\mathrm{HgCl}_{2}\left\{\mathrm{Fe}\left(\mathrm{C}_{5} \mathrm{H}_{4} \mathrm{PC} \mathrm{P}_{2}\right)_{2}\right\}\right] \quad[53]$, $\left[\mathrm{HgCl}_{2}\left\{\mathrm{Fe}\left(\mathrm{C}_{5} \mathrm{H}_{4} \mathrm{PPh}_{2}\right)_{2}\right\}\right]$ [84], and [ $\left.\mathrm{HgI}_{2}\left\{\mathrm{Fe}\left(\mathrm{C}_{5} \mathrm{H}_{4} \mathrm{PPh}_{2}\right)_{2}\right\}\right]$ [85]. 1-(Diphenylphosphino)ferrocenecarboxylic acid (Hdpf) behaves as a neutral P-donor phosphine towards mercury(II) halides, forming $\left[\mathrm{HgX}_{2}(\mathrm{Hdpf})_{2}\right]$ and $\left[\mathrm{HgX}(\mathrm{Hdpf})(\mu-\mathrm{X})_{2} \mathrm{HgX}(\mathrm{Hdpf})\right](\mathrm{X}=\mathrm{Cl}, \mathrm{Br}$ or I) [86]. Mercury(II) halide complexes of several ferrocene based pyridylphosphines have been structurally characterised (Table 4) [58]; depending upon the ligand and the reaction conditions various coordination modes can be accessed-monodentate P-donor, $\mathrm{P}, \mathrm{N}$-chelate and $\mathrm{P}, \mathrm{N}$-bridging modes.

The triphosphine $\mathrm{Ph}_{2} \mathrm{PCH}_{2} \mathrm{P}(\mathrm{Ph}) \mathrm{CH}_{2} \mathrm{PPh}_{2}$ forms $1: 1$ complexes with $\mathrm{HgBr}_{2}$ and $\mathrm{HgI}_{2}$, which on the evidence of their ${ }^{31} \mathrm{P}\left\{{ }^{1} \mathrm{H}\right\}$ NMR spectra were assigned as tetrahedral chelate complexes with the central $\mathrm{PhP}$ group uncoordinated. In marked contrast, $\mathrm{HgCl}_{2}$ gave the trimercury cation $\left[\mathrm{Hg}_{3} \mathrm{Cl}_{2}(\mu-\mathrm{Cl})_{2}\left\{\mathrm{PhP}\left(\mathrm{CH}_{2} \mathrm{PPh}_{2}\right)_{2}\right\}_{2}\right] \mathrm{Cl}\left(\mathrm{HCO}_{3}\right)$ (Fig. 11) in which all three phosphorus donor atoms are coordinated to different mercury centres [87].

The tetraphosphine cis,trans,cis-tetrakis(diphenylphosphino) cyclobutane $\left(\mathrm{L}^{\mathrm{b}}\right)$ binds to mercury salts in a $\kappa^{2} \kappa^{\prime 2}$ mode in $\left[\mathrm{Hg}_{2} \mathrm{X}_{4} \mathrm{~L}^{\mathrm{b}}\right]\left(\mathrm{X}=\mathrm{Cl}, \mathrm{Br}, \mathrm{CN}, \mathrm{NO}_{3}\right)$ [88]. One nitrate group can be displaced from each mercury by phosphines to give a trans- $\left[\mathrm{Hg}_{2}\left(\mathrm{NO}_{3}\right)_{2} \mathrm{~L}^{\mathrm{b}}\left(\mathrm{PR}_{3}\right)_{2}\right]^{2+}$ cation $\left(\mathrm{R}=\mathrm{Ph}, \mathrm{Bz}\right.$ or $\mathrm{CH}_{2} \mathrm{CH}_{2} \mathrm{CN}$, $\mathrm{PR}_{3}=\kappa^{1}-\mathrm{Ph}_{2} \mathrm{PCH}_{2} \mathrm{PPh}_{2}$ ).

The potentially tetradentate $\mathrm{N}\left(\mathrm{CH}_{2} \mathrm{CH}_{2} \mathrm{PPh}_{2}\right)_{3}$ gives tetrahedral $\left[\mathrm{HgX}\left\{\mathrm{N}\left(\mathrm{CH}_{2} \mathrm{CH}_{2} \mathrm{PPh}_{2}\right)_{3}\right\}\right] \mathrm{X}(\mathrm{X}=\mathrm{Cl}, \mathrm{Br}$ or I $)$ in which the nitrogen is uncoordinated $\left(\mathrm{Hg} \ldots{ }^{\bullet} \mathrm{N}=3.1 \AA\right)$ [89]. This tripodal ligand has also given a series of organomercury(II) complexes $\left[\mathrm{HgR}\left\{\mathrm{N}\left(\mathrm{CH}_{2} \mathrm{CH}_{2} \mathrm{PPh}_{2}\right)_{3}\right\}\right]\left[\mathrm{O}_{3} \mathrm{SCF}_{3}\right]\left(\mathrm{R}=\mathrm{Me}, \mathrm{Et}, \mathrm{Ph}, \mathrm{C}_{6} \mathrm{~F}_{5}\right)$ which are distorted tetrahedral $\left(\mathrm{P}_{3} \mathrm{C}\right)$, which contrasts with the linear coordination favoured by organomercury salts [90-93]. The Hg- $\mathrm{C}$ bond is readily broken on reaction with thiols, acetic- or trifluoroacetic acids, to form $\left[\mathrm{HgY}\left\{\mathrm{N}\left(\mathrm{CH}_{2} \mathrm{CH}_{2} \mathrm{PPh}_{2}\right)_{3}\right\}\right]^{+}\left(\mathrm{Y}=\mathrm{RS}, \mathrm{MeCO}_{2}, \mathrm{CF}_{3} \mathrm{SO}_{3}\right)$ and $\mathrm{RH}$. The related tetraphosphine ligand $\mathrm{P}\left(\mathrm{CH}_{2} \mathrm{CH}_{2} \mathrm{PPh}_{2}\right)_{3}$ also forms $\left[\mathrm{HgR}\left\{\mathrm{P}\left(\mathrm{CH}_{2} \mathrm{CH}_{2} \mathrm{PPh}_{2}\right)_{3}\right\}\right] \mathrm{BF}_{4}(\mathrm{R}=\mathrm{Me}$ or $\mathrm{Ph})$; the structure of the former shows a near-linear coordination to the central phosphorus and the Me group, with weaker interactions with two of the pendant phosphines (Fig. 12) [94].

Although mercurous ions $[\mathrm{Hg}-\mathrm{Hg}]^{2+}$ usually disproportionate on treatment with phosphines, there are subvalent clusters $\left[\mathrm{Hg}_{3}\right]^{4+}$ stabilised by diphosphinomethane ligands. Treatment of $[\mathrm{Hg}-\mathrm{Hg}]\left[\mathrm{CF}_{3} \mathrm{SO}_{3}\right]_{2}$ with $\mathrm{Me}_{2} \mathrm{PCH}_{2} \mathrm{PMe}_{2}$, or reaction of $\left[\mathrm{Hg}(\mathrm{dmso})_{6}\right]\left[\mathrm{CF}_{3} \mathrm{SO}_{3}\right]_{2}$ with metallic mercury and $\mathrm{Me}_{2} \mathrm{PCH}_{2} \mathrm{PMe}_{2}$, produces the triangular cluster $\left[\mathrm{Hg}_{3}\left(\mathrm{Me}_{2} \mathrm{PCH}_{2} \mathrm{PMe}_{2}\right)_{4}\right]\left[\mathrm{CF}_{3} \mathrm{SO}_{3}\right]_{4}$ with the structure shown in Fig. 13. [95]. The related $\left[\mathrm{Hg}_{3}\left(\mathrm{Ph}_{2} \mathrm{PCH}_{2} \mathrm{PPh}_{2}\right)_{3}\right][\mathrm{Y}]_{4}\left(\mathrm{Y}=\mathrm{PF}_{6}, \mathrm{O}_{3} \mathrm{SCF}_{3}, 1 / 2 \mathrm{SiF}_{6}, 1 / 2 \mathrm{SO}_{4}\right)$ also contain an $\mathrm{Hg}_{3}$ triangle, but with only three diphosphinomethane ligands, one on each edge (Fig. 14) [96]. Halide ions react with the cluster to form poor yields of $\left[\mathrm{Hg}_{3}\left(\mathrm{Ph}_{2} \mathrm{PCH}_{2} \mathrm{PPh}_{2}\right)_{3} \mathrm{X}_{n}\right]^{(4-n)+}$ ( $n=1$ or 2 ) along with elemental $\mathrm{Hg}$ and $\mathrm{Hg}$ (II) complexes, but the thiocyanate species $\left[\mathrm{Hg}_{3}\left(\mathrm{Ph}_{2} \mathrm{PCH}_{2} \mathrm{PPh}_{2}\right)_{3}(\mathrm{SCN})_{2}\right]\left[\mathrm{CF}_{3} \mathrm{SO}_{3}\right]_{2}$ has been structurally characterised [97]. The diarsine analogue $\left[\mathrm{Hg}_{3}\left(\mathrm{Ph}_{2} \mathrm{AsCH}_{2} \mathrm{AsPh}_{2}\right)_{3}\right]\left[\mathrm{CF}_{3} \mathrm{SO}_{3}\right]_{4}$ [98] and the mixed metal $\left[\mathrm{AgHg}_{2}\left(\mathrm{Ph}_{2} \mathrm{PCH}_{2} \mathrm{PPh}_{2}\right)_{3}\right][\mathrm{Y}]_{3}[99]$ are also known.

\section{Group 13}

Boron and aluminium halides are textbook examples of Lewis acids, much studied and widely used industrially. Gallium(III) and indium(III) halides function similarly, although their uses are much less widespread, whilst the Lewis acidity of thallium(III) is very limited.

\subsection{Boron}

Phosphine-borane adducts were the subject of two comprehensive reviews in 2010 [10,11], and hence we restrict discussion to adducts of the boron halides $\mathrm{BX}_{3}(\mathrm{X}=\mathrm{F}, \mathrm{Cl}, \mathrm{Br}$ or I). The synthesis of $\mathrm{X}_{3} \mathrm{~B}-\mathrm{PR}_{3}$ adducts dates back over 100 years, with much experimental work carried out in the 1960-1980 period. In the process of adduct formation, the trigonal planar $\mathrm{BX}_{3}$ unit is converted into a pyramidal fragment (an endothermic process 


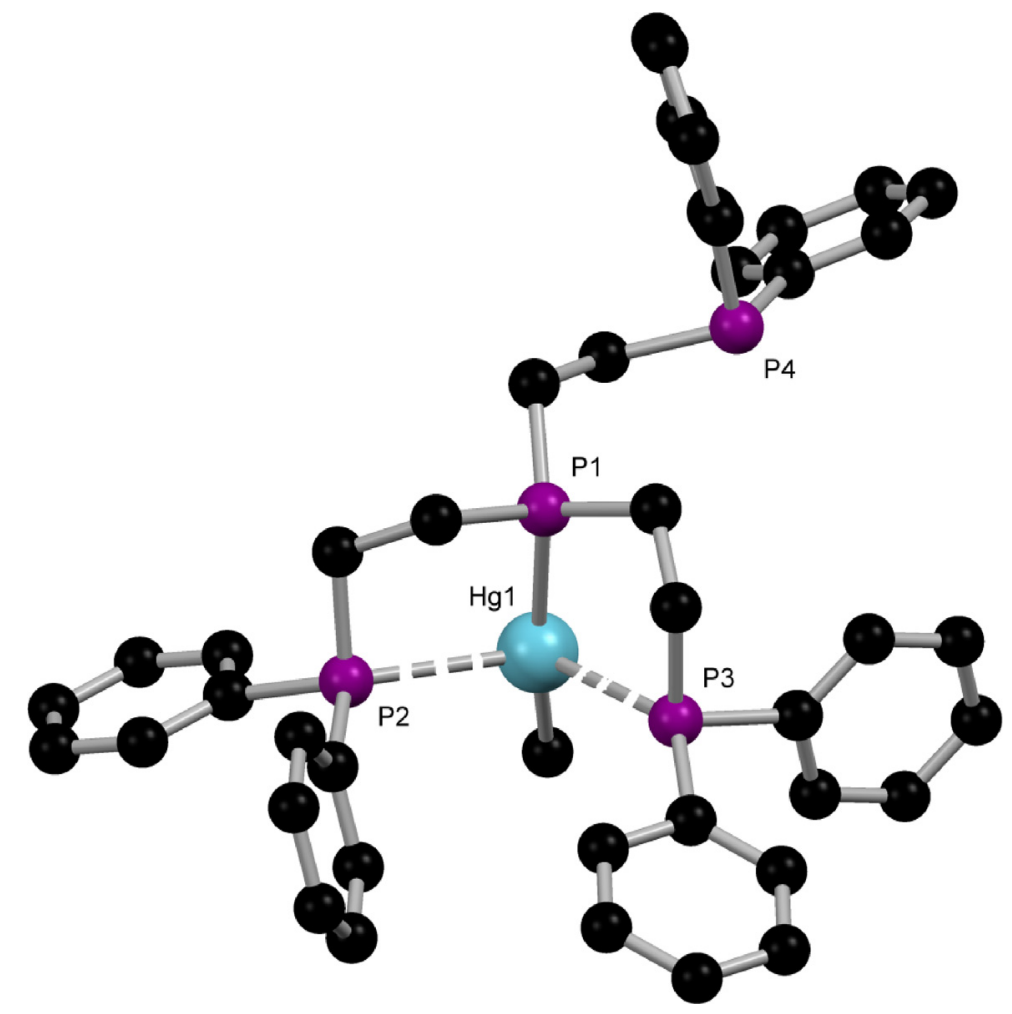

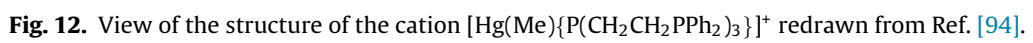

variously described as the deformation or reorganisation energy) and the Lewis acid-Lewis base bond forms (an exothermic process). Many textbooks state that Lewis acidity increases $\mathrm{BF}_{3}<\mathrm{BCl}_{3}<\mathrm{BBr}_{3}$ (often generalised without explanation, but traceable back to the stability of the $\mathrm{NH}_{3}$ adducts). This is counter-intuitive on electronegativity grounds, and was rationalised as being due to hyperconjugation $-\mathrm{p} \pi-\mathrm{p} \pi$ bonding between the empty boron $\mathrm{p}$ orbital and the filled p orbitals on the halogen, which was assumed to give the best overlap with the lighter halogens and hence make $\mathrm{BF}_{3}$ in particular a weaker acceptor towards the Lewis base. Since the $\pi$-bonding would be disrupted this would also make pyramidalisation more endothermic. In the last 15 years much

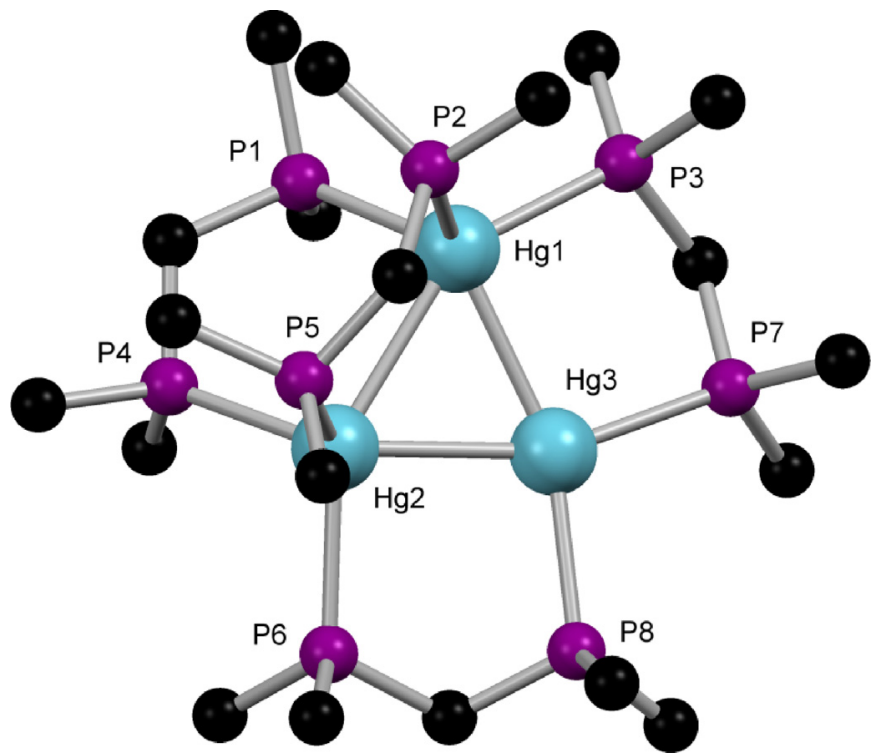

Fig. 13. View of the structure of the cation $\left[\mathrm{Hg}_{3}\left(\mathrm{Me}_{2} \mathrm{PCH}_{2} \mathrm{PMe}_{2}\right)_{4}\right]^{4+}$ redrawn from Ref. [95]. computational effort has been devoted to exploring the factors involved, the majority of the work dealing with amine adducts of $\mathrm{BX}_{3}$ or $\mathrm{BH}_{3}$. The detailed and somewhat subtle conclusions show considerable dependence on the level and model of calculations employed, and have often proved controversial in detail. These are well summarised in $[10,107]$ and some key contributions are in [108-112] and refs therein. Much of this work deals with

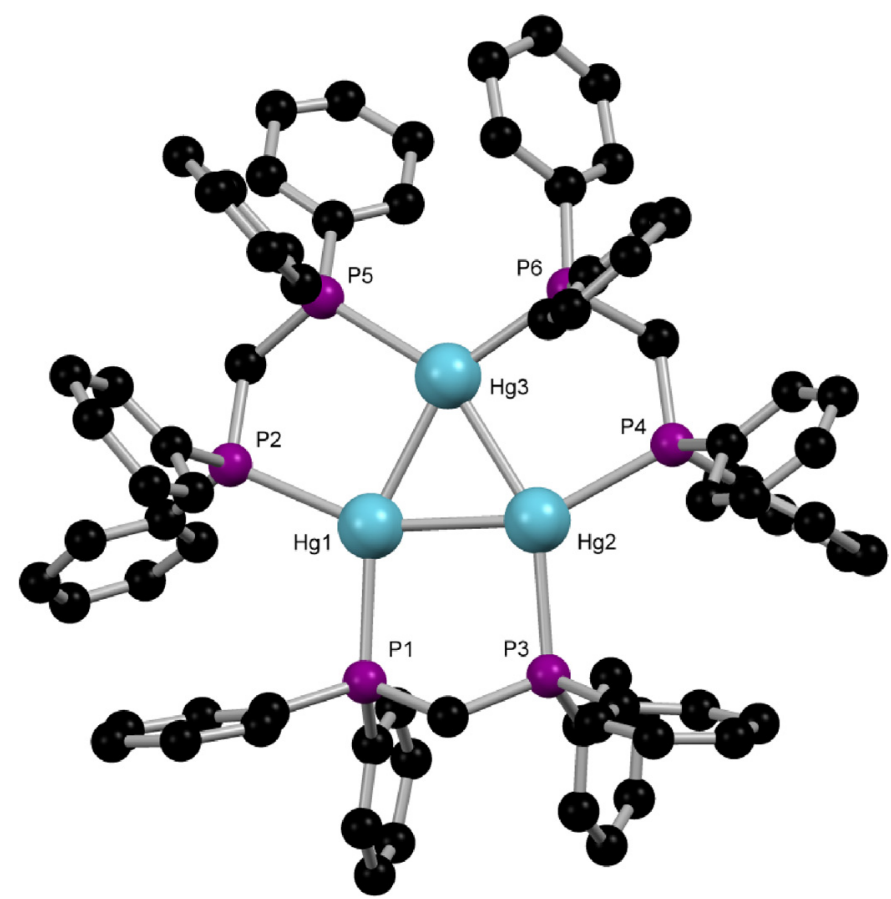

Fig. 14. View of the structure of the cation $\left[\mathrm{Hg}_{3}\left(\mathrm{Ph}_{2} \mathrm{PCH}_{2} \mathrm{PPh}_{2}\right)_{3}\right]^{4+}$ redrawn from Ref. [96]. 


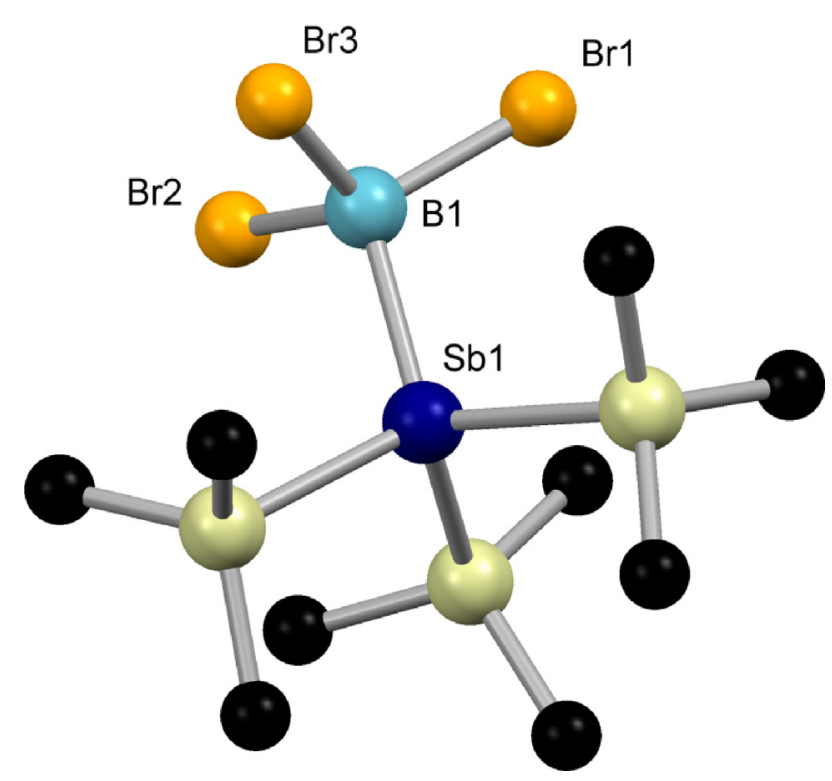

Fig. 15. View of the structure of $\left[\mathrm{BBr}_{3}\left\{\mathrm{Sb}\left(\mathrm{SiMe}_{3}\right)_{3}\right\}\right]$ redrawn from Ref. [127].

complexes that lie outside the present article, but some points relevant to phosphine/arsine adducts are:

- the Lewis acid-Lewis base interaction depends upon the particular pair involved, and discussing one component in isolation can be misleading (but surprisingly often done);

- $\mathrm{p} \pi-\mathrm{p} \pi$ hyperconjugation is not responsible for the observed trends, which arise via the $\sigma$-bonding interactions and orbital energies of $\mathrm{B}-\mathrm{X}$ and $\mathrm{B}-\mathrm{P}$ bonds;

- there is a subtle interplay between substituent size and electronegativity on the boron Lewis acidity;

- the bond dissociation energy is not a good measure of intrinsic donor-acceptor strength;

- the modelling usually deals with gas phase molecules and does not take into account solvation effects in solution, or packing and intermolecular interactions in the solids.

Boron halide adducts made in the gas phase or isolated in low temperature matrices have been reviewed, along with the relevant computational modelling [12].

Boron trihalides form 1:1 complexes with phosphine and arsine ligands, usually made by combination of the constituents in an inert solvent; hexane, benzene or toluene are the most commonly used, or sometimes in the absence of a solvent [113-126]. Table 5 lists structural data on typical examples. All examples are based upon a $\mathrm{C}_{3 \mathrm{v}}$ boron centre and the $\mathrm{d}(\mathrm{B}-\mathrm{P} / \mathrm{As})$ bond lengths for a common soft Lewis base decrease $\mathrm{Cl}>\mathrm{Br}>\mathrm{I}$. The only stibine examples for which structural data are available, $\left[\mathrm{BX}_{3}\left\{\mathrm{Sb}\left(\mathrm{SiMe}_{3}\right)_{3}\right\}\right](\mathrm{X}=\mathrm{Cl}, \mathrm{Br}$ or I) (Fig. 15) have B-Sb distances which are effectively the same (within experimental error), and data on other stibine adducts are required before any conclusions can be drawn.

The $\mathrm{PR}_{3}$ or $\mathrm{AsR}_{3}$ (where $\mathrm{R}$ is a simple alkyl or aryl group) complexes are stable molecular monomers that have attracted a good deal of spectroscopic study, especially by IR/Raman and multinuclear NMR $\left({ }^{1} \mathrm{H},{ }^{13} \mathrm{C},{ }^{11} \mathrm{~B},{ }^{31} \mathrm{P}\left\{{ }^{1} \mathrm{H}\right\}\right)$ methods-in general the data are unexceptional and the trends with changing $\mathrm{X}$ or $\mathrm{ER}_{3}$ are systematic $[113,124,128,129]$. Adducts with $\mathrm{PX}_{3}(\mathrm{X}=\mathrm{Cl}, \mathrm{Br}$ or I $)$ are also known [116,117]; these are less stable than $\mathrm{PR}_{3}$ complexes and have markedly longer $\mathrm{P}-\mathrm{B}$ bonds. Trivinylphosphine forms $\left[\mathrm{BX}_{3}\left\{\mathrm{P}\left(\mathrm{CH}=\mathrm{CH}_{2}\right)_{3}\right\}\right](\mathrm{X}=\mathrm{Cl}, \mathrm{Br}$ or $\mathrm{I})$ by direct reaction with $\mathrm{BX}_{3}$ at low temperatures, but the corresponding reaction of $\left[\mathrm{BF}_{3}\left(\mathrm{OEt}_{2}\right)\right]$ and $\mathrm{P}\left(\mathrm{CH}=\mathrm{CH}_{2}\right)_{3}$ gave polymeric materials [113]. The only structurally characterised $\mathrm{BF}_{3}-$ phosphine complex is $\left[\mathrm{BF}_{3}\left(\mathrm{PEt}_{3}\right)\right]$, which is stable at room temperature, but which has a long $\mathrm{B}-\mathrm{P}$ bond [113]. The secondary phosphine adducts $\left[\mathrm{BX}_{3}\left(\mathrm{PH}^{\mathrm{t}} \mathrm{Bu}_{2}\right)\right]$ $(\mathrm{X}=\mathrm{Cl}$ or $\mathrm{Br}$ ) are unstable in air and convert to the phosphine oxides $\left[\mathrm{BX}_{3}\left(\mathrm{OPH}^{\mathrm{t}} \mathrm{Bu}_{2}\right)\right]$, whilst $\mathrm{LiN}\left(\mathrm{SiMe}_{3}\right)_{2}$ caused deprotonation to form $\left[\left\{\mathrm{BX}_{2}\left(\mathrm{P}^{t} \mathrm{Bu}_{2}\right)\right\}_{2}\right][122]$. The silyl-phosphine and -arsine complexes $\left[\mathrm{BX}_{3}\left\{\mathrm{E}\left(\mathrm{SiMe}_{3}\right)_{3}\right\}\right](\mathrm{X}=\mathrm{Cl}, \mathrm{Br}$ or I; $\mathrm{E}=\mathrm{P}$ or $\mathrm{As})$ were made by combination of the constituents at low temperatures, but at higher temperatures $\mathrm{Si}-\mathrm{E}$ cleavage occurs to give $\left[\left\{\mathrm{BX}_{2}\left(\mathrm{E}\left(\mathrm{SiMe}_{3}\right)_{2}\right)\right\}_{2}\right]$ which can be made directly from the appropriate boron halide and $\mathrm{Li}\left[\mathrm{E}\left(\mathrm{SiMe}_{3}\right)_{2}\right][121,125,126]$.

\subsection{Aluminium}

There are considerable similarities between adducts formed by $\mathrm{AlX}_{3}(\mathrm{X}=\mathrm{Cl}, \mathrm{Br}$ or $\mathrm{I})$ and those of $\mathrm{BX}_{3}$ already discussed, but also some notable differences. Whilst tetrahedral four coordination is still the norm, five- or six-coordination is found in some aluminium complexes. Very few complexes (none with soft donors) are known for $\mathrm{AlF}_{3}$, which unlike the molecular $\mathrm{BF}_{3}$ is an inert fluorine-bridged polymer [130]. Monodentate $\mathrm{ER}_{3}$ adducts of $\mathrm{AlR}_{3}{ }_{3}\left(\mathrm{R}^{\prime}=\mathrm{alkyl}\right)$ have been thoroughly investigated, but detailed studies of complexes of $\mathrm{AlX}_{3}$ are surprisingly rare and complexes with bi- and polydentates have received very limited study. Anionic mixed-donor polydentates containing- $-\mathrm{PR}_{2}$ groups are better known. Stibine and even bismuthine complexes have been obtained with trialkylaluminiums. In addition to their inherent interest, several series of adducts have been explored as precursors for MOCVD (metal organic chemical vapour deposition) of the III-V materials $\mathrm{AlE}(\mathrm{E}=\mathrm{P}-\mathrm{Bi})$, which are direct band gap semi-conductors, whilst other aluminium-phosphine adducts have been isolated (sometimes serendipitously) from catalytic systems that have been activated with alkylaluminiums. There is also a large amount of chemistry on aluminium dimers with $\mathrm{ER}_{2}{ }^{-}$bridges which fall outside the scope of the present article; again the driving force for much of this work was reagents for the production of III-V semiconductors.

The various energy contributions to the formation of $\mathrm{X}_{3} \mathrm{Al}-\mathrm{ER}_{3}$ adducts follow closely from those discussed under boron above. The theoretical investigations have shown that whilst bond dissociation energies may be larger for aluminium than for the corresponding boron complex, this is a result of less energy needed to convert the planar $\mathrm{AlX}_{3}$ fragment to the pyramidal shape, rather than to inherently stronger donor-acceptor bonds [110]. An overview of the data indicates that despite some anomalies, the conclusion of decreasing Lewis acid strength is in the order $\mathrm{AlCl}_{3}>\mathrm{AlBr}_{3}>\mathrm{AlI}_{3}$, and also an order $\mathrm{AlCl}_{3}>\mathrm{AlBr}_{3}>\mathrm{GaCl}_{3}>\mathrm{GaBr}_{3}[131]$. Note these are the reverse of that observed for boron halides, although more structural data on series of boron halide phosphine/arsine adducts are desirable to support the conclusions. The calculations are performed for gas phase molecules and do not take into account other (usually small) energy terms such as solvation or lattice energies. However, such small terms can result in significant effects, which may complicate or even obscure the underlying trends. For example, it has been proposed that some apparently anomalous results in the experimental data from some solid aluminium adducts could be ascribed to solid state effects such as H-bonding or intermolecular packing [131]. Crystallographically characterised complexes are listed in Table 6.

During studies of low valent aluminium halides, Schnoeckel and co-workers obtained crystal structures of $\left[\mathrm{Al}_{4} \mathrm{I}_{4}\left(\mathrm{PEt}_{3}\right)_{4}\right]$ (formally $\mathrm{Al}(\mathrm{I})$ ) and $\left[\mathrm{Al}_{2} \mathrm{I}_{4}\left(\mathrm{PEt}_{3}\right)_{2}\right]$ (formally $\mathrm{Al}(\mathrm{II})$ ), as well as of the five-coordinate trans-trigonal bipyramidal $\left[\mathrm{AlI}_{3}\left(\mathrm{PEt}_{3}\right)_{2}\right]$ (Fig. 16) $[132,133]$. In $\left[\mathrm{Al}_{4} \mathrm{I}_{4}\left(\mathrm{PEt}_{3}\right)_{4}\right]$ there is a planar $\mathrm{Al}_{4}$ ring with $\mathrm{Al}-\mathrm{Al}=2.597(3) \AA$ (Fig. 17), whilst $\left[\mathrm{Al}_{2} \mathrm{I}_{4}\left(\mathrm{PEt}_{3}\right)_{2}\right]$ is a dimer with an unbridged $\mathrm{Al}-\mathrm{Al}$ bond (2.546(3) $\mathrm{A}$ ) (Fig. 18). The tetrahedral $\left[\mathrm{AlX}_{3}\left(\mathrm{ER}_{3}\right)\right]\left(\mathrm{ER}_{3}=\mathrm{PPh}_{3}, \mathrm{AsMe}_{3}\right.$ or AsEt 3$)[124,131]$ have ${ }^{27} \mathrm{Al} \mathrm{NMR}$ 
Table 5

X-ray structures of boron halide adducts.

\begin{tabular}{|c|c|c|c|}
\hline Complex & $\mathrm{B}-\mathrm{P} / \mathrm{As} / \mathrm{Sb}(\AA)$ & $\mathrm{B}-\mathrm{X}(\AA)$ & Ref. \\
\hline$\left[\mathrm{BF}_{3}\left(\mathrm{PEt}_{3}\right)\right]$ & $2.028(1)$ & $1.378(2)-1.384(2)$ & [113] \\
\hline$\left[\mathrm{BCl}_{3}\left(\mathrm{PMe}_{3}\right)\right]$ & $1.957(5)$ & $1.850(3)-1.866(5)$ & {$[114]$} \\
\hline$\left[\mathrm{BBr}_{3}\left(\mathrm{PMe}_{3}\right)\right]$ & $1.924(12)$ & $2.016(10)-2.025(6)$ & {$[114]$} \\
\hline$\left[\mathrm{BI}_{3}\left(\mathrm{PMe}_{3}\right)\right]$ & $1.918(15)$ & $2.272(16)-2.237(9)$ & [114] \\
\hline$\left[\mathrm{BBr}_{3}\left(\mathrm{P}^{n} \mathrm{Pr}_{3}\right)\right]$ & $1.95(1)$ & $2.009(3)$ & [116] \\
\hline$\left[\mathrm{BI}_{3}\left(\mathrm{PCy}_{3}\right)\right]$ & $1.972(3)$ & $2.236(3)-2.249(3)$ & [119] \\
\hline$\left[\mathrm{BCl}_{3}\left(\mathrm{PMe}_{2} \mathrm{Ph}\right)\right]$ & $1.963(2)$ & $1.836(2)-1.839(2)$ & [115] \\
\hline$\left[\mathrm{BBr}_{3}\left(\mathrm{PMePh}_{2}\right)\right]$ & $1.976(9)$ & $2.004(11)-2.046(12)$ & [120] \\
\hline$\left[\mathrm{BBr}_{3}\left(\mathrm{Ph}_{2} \mathrm{PH}\right)\right]$ & $1.965(4)$ & $1.997(4)-2.013(4)$ & {$[120]$} \\
\hline$\left[\mathrm{BCl}_{3}\left\{\mathrm{P}\left(\mathrm{CH}=\mathrm{CH}_{2}\right)_{3}\right\}\right]$ & $1.970(2)$ & $1.841(3)-1.847(2)$ & [113] \\
\hline$\left[\mathrm{BBr}_{3}\left\{\mathrm{P}\left(\mathrm{CH}=\mathrm{CH}_{2}\right)_{3}\right\}\right]$ & $1.952(2)$ & $2.004(2)-2.009(2)$ & [113] \\
\hline$\left[\mathrm{BI}_{3}\left\{\mathrm{P}\left(\mathrm{CH}=\mathrm{CH}_{2}\right)_{3}\right\}\right]$ & $1.944(4)$ & $2.226(5)-2.232(4)$ & [113] \\
\hline$\left[\mathrm{BBr}_{3}\left(\mathrm{PI}_{3}\right)\right]$ & $2.01(1)$ & $2.387(3)-2.392(1)$ & [116] \\
\hline$\left[\mathrm{BBr}_{3}\left(\mathrm{PBr}_{3}\right)\right]$ & $2.01(2)$ & $1.96(2)-1.99(1)$ & {$[116]$} \\
\hline$\left[\mathrm{BCl}_{3}\left(\mathrm{PPh}_{3}\right)\right]$ & $2.006(3)$ & $1.835(3)-1.852(2)$ & [115] \\
\hline$\left[\mathrm{BBr}_{3}\left(\mathrm{PPh}_{3}\right)\right]$ & $1.99(1)$ & $1.978(1)-2.013(9)$ & [118] \\
\hline$\left[\mathrm{BCl}_{3}\left\{\mathrm{P}\left(\mathrm{SiMe}_{3}\right)_{3}\right\}\right]$ & $2.022(9)$ & $1.841(9)-1.857(9)$ & [121] \\
\hline$\left[\mathrm{BBr}_{3}\left\{\mathrm{P}\left(\mathrm{SiMe}_{3}\right)_{3}\right\}\right]$ & $1.998(2)$ & $1.995(20)-2.035(20)$ & [121] \\
\hline$\left[\mathrm{BBr}_{3}\left(\mathrm{PH}^{t} \mathrm{Bu}_{2}\right)\right]$ & $2.01(1), 2.00(1)$ & $2.01(2), 2.02(2)$ & {$[122]$} \\
\hline$\left[\left(\mathrm{BCl}_{3}\right)_{2}\left(\mu-\mathrm{Ph}_{2} \mathrm{PCH}_{2} \mathrm{PPh}_{2}\right)\right]$ & $1.995(3), 1.980(3)$ & $1.835(3)-1,850(3)$ & [115] \\
\hline$\left[\mathrm{BCl}_{3}\left(\mathrm{AsMe}_{3}\right)\right]$ & $2.065(6)$ & $1.828(7)-1.830(4)$ & [123] \\
\hline$\left[\mathrm{BBr}_{3}\left(\mathrm{AsMe}_{3}\right)\right]$ & $2.04(1)$ & $1.99(1)-2.04(1)$ & [123] \\
\hline$\left[\mathrm{BI}_{3}\left(\mathrm{AsMe}_{3}\right)\right]$ & $2.03(1)$ & $2.22(1)-2.23(1)$ & [123] \\
\hline$\left[\mathrm{BPh}_{3}\left(\mathrm{AsMe}_{3}\right)\right]$ & $2.148(3)$ & - & [124] \\
\hline$\left[\mathrm{B}\left(\mathrm{C}_{6} \mathrm{~F}_{5}\right)_{3}\left(\mathrm{AsEt}_{3}\right)\right]$ & $2.1905(18)$ & - & [124] \\
\hline$\left[\mathrm{BCl}_{3}\left\{\mathrm{As}\left(\mathrm{SiMe}_{3}\right)_{3}\right\}\right]$ & $2.118(6)$ & $1.822(7)-1.838(7)$ & {$[125]$} \\
\hline$\left[\mathrm{BBr}_{3}\left\{\mathrm{As}\left(\mathrm{SiMe}_{3}\right)_{3}\right\}\right]$ & $2.108(11)$ & $1.996(10)-2.034(10)$ & [126] \\
\hline$\left[\mathrm{BI}_{3}\left\{\mathrm{As}\left(\mathrm{SiMe}_{3}\right)_{3}\right\}\right]$ & $2.104(15)$ & $2.216(14)-2.262(14)$ & [125] \\
\hline$\left[\mathrm{BCl}_{3}\left\{\mathrm{Sb}\left(\mathrm{SiMe}_{3}\right)_{3}\right\}\right]$ & $2.259(21)$ & $1.842(21)-1.864(21)$ & [127] \\
\hline$\left[\mathrm{BBr}_{3}\left\{\mathrm{Sb}\left(\mathrm{SiMe}_{3}\right)_{3}\right\}\right]$ & $2.268(17)$ & $2.008(17)-2.032(17)$ & [127] \\
\hline$\left[\mathrm{BI}_{3}\left\{\mathrm{Sb}\left(\mathrm{SiMe}_{3}\right)_{3}\right\}\right]$ & $2.257(8)$ & $2.231(8)-2.259(8)$ & [127] \\
\hline
\end{tabular}

chemical shifts in the range $\delta \sim 100-120$, typical of four-coordinate aluminium [124]. Analogous to the boron halide complexes, $\left[\mathrm{AlX}_{3}\left(\mathrm{PH}^{t} \mathrm{Bu}_{2}\right)\right]$ are dioxygen sensitive and readily convert into $\left[\mathrm{AlBr}_{3}\left(\mathrm{OPH}^{t} \mathrm{Bu}_{2}\right)\right]$ on exposure to air [122]. The very sterically hindered $\mathrm{P}(\text { mesityl })_{3}$ forms unstable adducts $\left[\mathrm{AlX}_{3}\left\{\mathrm{P}\left(\right.\right.\right.$ mesityl $\left.\left._{3}\right\}\right]$ $(\mathrm{X}=\mathrm{Cl}$ or $\mathrm{Br}$ ), with very long $\mathrm{Al}-\mathrm{P}$ bonds (see Table 6 ) and the solution NMR data show extensive dissociation [134]. In solution they

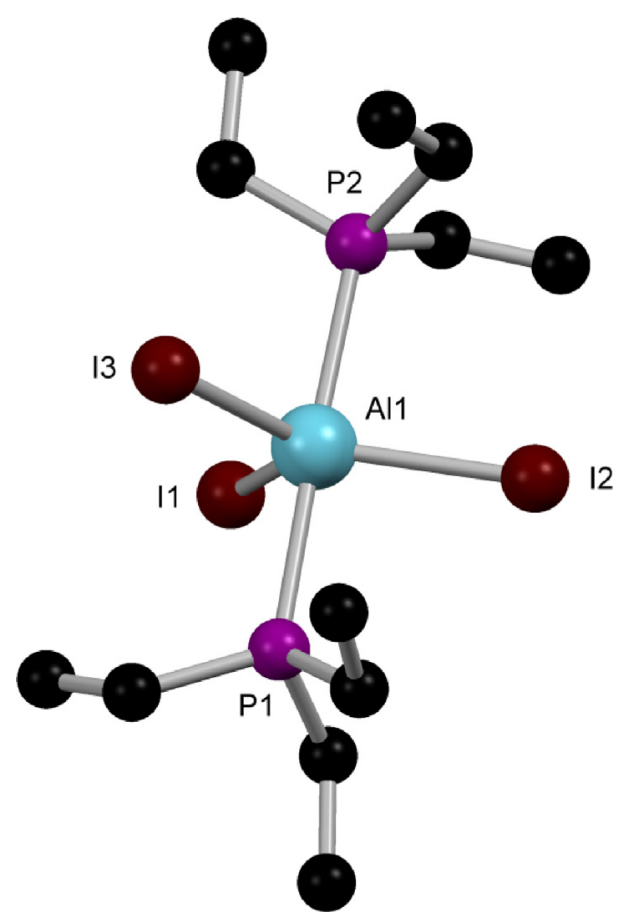

Fig. 16. View of the structure of $\left[\mathrm{AlI}_{3}\left(\mathrm{PEt}_{3}\right)_{2}\right]$ redrawn from Ref. [132]. behave as frustrated Lewis pairs, activating $\mathrm{CO}_{2}$ towards reduction to $\mathrm{MeOH}$ by ammonia borane. Reaction of $\mathrm{P}\left(\mathrm{SiMe}_{3}\right)_{3}$ with $\mathrm{AlX}_{3}(\mathrm{X}=\mathrm{Cl}$ or $\mathrm{Br})$ in toluene produces $\left[\mathrm{AlX}_{3}\left\{\mathrm{P}\left(\mathrm{SiMe}_{3}\right)_{3}\right\}\right]$ with no dehalosilylation (- $\mathrm{Me}_{3} \mathrm{SiCl}$ ) observed [135].

Alane $\left(\mathrm{AlH}_{3}\right)$ forms very unstable complexes with small phosphines which lose phosphine and form $\left[\left\{\mathrm{AlH}_{3}\right\}_{n}\right]$, but with bulky phosphines or diphosphines more stable four- or five-coordinate adducts form [136,137]. Treatment of $\mathrm{LiAlH}_{4}$ in diethyl ether

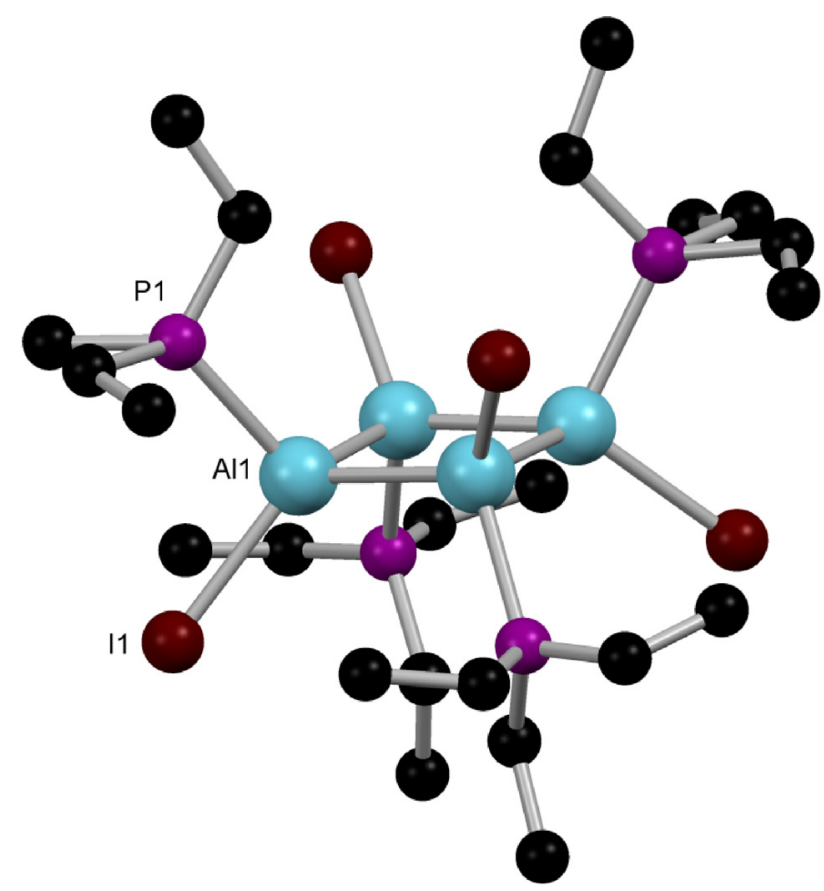

Fig. 17. View of the structure of $\left[\mathrm{Al}_{4} \mathrm{I}_{4}\left(\mathrm{PEt}_{3}\right)_{4}\right]$ redrawn from Ref. [132]. 
Table 6

X-ray structures of aluminium complexes.

\begin{tabular}{|c|c|c|c|c|}
\hline Complex & $\mathrm{Al}-\mathrm{P} / \mathrm{As} / \mathrm{Sb}(\AA)$ & $\mathrm{Al}-\mathrm{X}(\AA ̊)$ & Comments & Ref. \\
\hline$\left[\mathrm{AlBr}_{3}\left(\mathrm{PPh}_{3}\right)\right]$ & $2.4296(15)$ & $2.1079(13)-2.1224(13)$ & & [131] \\
\hline$\left[\mathrm{AlCl}_{3}\left(\mathrm{AsPh}_{3}\right)\right]$ & $2.5191(9)$ & $2.0711(11)-2.1446(11)$ & & [124] \\
\hline$\left[\mathrm{AlI}_{3}\left(\mathrm{AsPh}_{3}\right)\right]$ & $2.5140(10)$ & $2.4985(3)$ & & [124] \\
\hline$\left[\mathrm{AlBr}_{3}\left(\mathrm{PH}^{t} \mathrm{Bu}_{2}\right)\right]$ & $2.439(3), 2.441(2)$ & $2.283(2), 2.286(2)$ & & [122] \\
\hline$\left[\mathrm{AlI}_{3}\left(\mathrm{PEt}_{3}\right)_{2}\right]$ & $2.520(4), 2.528(4)$ & $2.598(3)-2.618(3)$ & Tbp trans axial P & [132] \\
\hline$\left[\mathrm{AlCl}_{3}\left\{\mathrm{P}(\text { mesityl })_{3}\right\}\right]$ & $2.5238(11)$ & $2.1237(13)-2.1345(12)$ & & [134] \\
\hline$\left[\mathrm{AlBr}_{3}\left\{\mathrm{P}(\text { mesityl })_{3}\right\}\right]$ & $2.5360(10)$ & $2.2924(9)-2.2986(9)$ & & [134] \\
\hline$\left[\mathrm{Al}_{2} \mathrm{I}_{4}\left(\mathrm{PEt}_{3}\right)_{2}\right]$ & $2.440(2)$ & $2.561(2), 2.562(2)$ & Al-Al bond & [133] \\
\hline$\left[\mathrm{Al}_{4} \mathrm{I}_{4}\left(\mathrm{PEt}_{3}\right)_{4}\right]$ & $2.403(2)$ & $2.630(2)$ & planar $\mathrm{Al}_{4}$ ring & [132] \\
\hline$\left[\mathrm{AlCl}_{3}\left\{\mathrm{P}\left(\mathrm{SiMe}_{3}\right)_{3}\right\}\right]$ & $2.392(4)$ & $2.112(4)-2.125(4)$ & & [135] \\
\hline$\left[\mathrm{AlBr}_{3}\left\{\mathrm{P}\left(\mathrm{SiMe}_{3}\right)_{3}\right\}\right]$ & $2.391(6)$ & $2.287(5)-2.290(5)$ & & [135] \\
\hline$\left[\mathrm{AlH}_{3}\left(\mathrm{PCy}_{3}\right)\right]$ & $2.467(1)$ & - & & [136] \\
\hline$\left[\mathrm{AlH} 3\left(\mathrm{P}^{t} \mathrm{Bu}_{3}\right)\right]$ & $2.471(3)$ & - & & [137] \\
\hline$\left[\left(\mathrm{AlH}_{3}\right)_{2}\left\{\mu-\mathrm{Cy}_{2} \mathrm{P}\left(\mathrm{CH}_{2}\right)_{2} \mathrm{PCy}_{2}\right\}\right]$ & $2.406(2)$ & - & 4-Coord Al & [136] \\
\hline$\left[\left(\mathrm{AlH}_{3}\right)_{2}\left\{\mu-{ }^{i} \mathrm{Pr}_{2} \mathrm{P}\left(\mathrm{CH}_{2}\right)_{2} \mathrm{P}^{i} \mathrm{Pr}_{2}\right\}\right]$ & $2.451(3)$ & - & 4-Coord Al & [137] \\
\hline$\left[\left\{\left(\mathrm{AlH}_{3}\right)_{2}\left\{\mu-{ }^{i} \operatorname{Pr} 2 \mathrm{P}(\mathrm{CH} 2) 2 \mathrm{P}^{i} \mathrm{Pr}_{2}\right\}\right\} \mathrm{n}\right]$ & $2.708(3), 2.755(3)$ & - & Polymer 5-coord Al & [136] \\
\hline$\left[\left\{\left(\mathrm{NMe}_{3}\right)\left(\mathrm{AlH}_{3}\right)\right\}_{2}\left\{\mu-\mathrm{Me}_{2} \mathrm{P}\left(\mathrm{CH}_{2}\right)_{2} \mathrm{PMe}_{2}\right)\right]$ & $2.688(5)$ & - & Dimer 5-coord Al & [138] \\
\hline$\left[\mathrm{AlMe}_{3}\left(\mathrm{PPh}_{3}\right)\right]$ & $2.535(1)$ or $2.5329(6)$ & - & & {$[140,141]$} \\
\hline$\left[\mathrm{AlMe}_{3}\left\{\mathrm{P}(\text { o-tolyl })_{3}\right\}\right]$ & $2.584(2)$ & - & & {$[140]$} \\
\hline$\left[\mathrm{AlEt}_{3}\left(\mathrm{PPh}_{3}\right)\right]$ & $2.5413(4)$ & - & & [141] \\
\hline$\left[\mathrm{Al}^{t} \mathrm{Bu}_{3}\left(\mathrm{PnPr}_{3}\right)\right]$ & $2.5943(3)$ & & & [142] \\
\hline$\left[\mathrm{Al}^{\mathrm{t}} \mathrm{Bu}_{3}\left(\mathrm{P}^{\mathrm{i}} \mathrm{Pr}_{3}\right)\right]$ & $2.667(2)$ & - & & [147] \\
\hline$\left[\left(\mathrm{AlMe}_{3}\right)_{2}\left(\mu-\mathrm{Ph}_{2} \mathrm{PCH}_{2} \mathrm{PPh}_{2}\right)\right]$ & $2.521(2), 2.585(2)$ & - & & [143] \\
\hline$\left[\left(\mathrm{Al}^{i} \mathrm{Bu}_{3}\right)_{2}\left(\mu-\mathrm{Ph}_{2} \mathrm{PCH}_{2} \mathrm{CH}_{2} \mathrm{PPh}_{2}\right)\right]$ & $2.560(2)$ & - & & [144] \\
\hline$\left[\left(\mathrm{AlCl}_{2} \mathrm{Me}\right)\left(\mathrm{AlCl}_{3}\right)\left(\mu-\mathrm{Ph}_{2} \mathrm{PCH}_{2} \mathrm{PPh}_{2}\right)\right]$ & $2.451(2), 2.497(2)$ & $2.020(4)-2.138(2)$ & & [145] \\
\hline$\left[\left(\mathrm{AlMe}_{3}\right)_{2}\left(\mu-\mathrm{Ph}_{2} \mathrm{PCH}_{2} \mathrm{CH}_{2} \mathrm{PPh}_{2}\right)\right]$ & $2.544(4)$ & & & [146] \\
\hline$\left[\left(\mathrm{AlMe}_{3}\right)_{3}\left\{\boldsymbol{\mu}-\left(\mathrm{Ph}_{2} \mathrm{PCH}_{2} \mathrm{CH}_{2}\right)_{2} \mathrm{PPh}\right\}\right]$ & $2.535(7), 2.531(7), 2.504(6)$ & - & & [146] \\
\hline$\left[\left(\mathrm{AlMe}_{3}\right)_{2}\left\{\mu-\mathrm{Ph}_{2} \mathrm{P}\left(\mathrm{CH}_{2}\right)_{3} \mathrm{PPh}_{2}\right)\right]$ & $2.5008(5)$ & - & & [141] \\
\hline$\left[\left(\mathrm{AlEt}_{3}\right)_{2}\left\{\mu-\mathrm{Ph}_{2} \mathrm{P}\left(\mathrm{CH}_{2}\right)_{3} \mathrm{PPh}_{2}\right)\right]$ & $2.5300(5)$ & - & & [141] \\
\hline$\left[\left(\mathrm{AlMe}_{3}\right)_{2}\left\{\mu-\left({ }^{t} \mathrm{BuHPCH}{ }_{2} \mathrm{CH}_{2} \mathrm{P}^{t} \mathrm{BuH}\right\}\right]\right.$ & $2.533(3)$ & - & & [159] \\
\hline$\left[\mathrm{Al}^{t} \mathrm{Bu}_{3}\left(\mathrm{As}^{\mathrm{i}} \mathrm{Pr}_{3}\right)\right]$ & $2.839(1)$ & - & & [147] \\
\hline$\left[\mathrm{AlEt}_{3}\left\{\mathrm{P}\left(\mathrm{SiMe}_{3}\right)_{3}\right\}\right]$ & $2.555(2)$ & - & & [147] \\
\hline$\left[\mathrm{AlEt}_{3}\left\{\mathrm{As}\left(\mathrm{SiMe}_{3}\right)_{3}\right\}\right]$ & $2.654(2)$ & - & & {$[147]$} \\
\hline$\left[\mathrm{Al}\left(\mathrm{Me}_{3} \mathrm{CCH}_{2}\right)_{3}\left\{\mathrm{As}\left(\mathrm{SiMe}_{3}\right)_{3}\right\}\right]$ & $2.72(2)$ & - & & [148] \\
\hline$\left[\mathrm{Al}\left(\mathrm{Me}_{3} \mathrm{CCH}_{2}\right)_{3}\left\{\mathrm{P}\left(\mathrm{SiMe}_{3}\right)_{3}\right\}\right]$ & $2.681(4)$ & - & & [148] \\
\hline$\left[\mathrm{Al}\left(\mathrm{Me}_{3} \mathrm{SiCH}_{2}\right)_{3}\left\{\mathrm{As}\left(\mathrm{SiMe}_{3}\right)_{3}\right\}\right]$ & $2.671(3)$ & - & & [148] \\
\hline$\left[\mathrm{Al}\left(\mathrm{Me}_{3} \mathrm{SiCH}_{2}\right)_{2} \mathrm{Br}\left\{\mathrm{P}\left(\mathrm{SiMe}_{3}\right)_{3}\right\}\right]$ & $2.498(7)$ & $2.351(5)$ & & [148] \\
\hline$\left[\mathrm{Al}^{t} \mathrm{Bu}_{3}\left(\mathrm{SbEt}_{3}\right)\right]$ & $2.8447(7)$ & - & & [150] \\
\hline$\left[\mathrm{Al}^{t} \mathrm{Bu}_{3}\left(\mathrm{Sb}^{i} \mathrm{Pr}_{3}\right)\right]$ & $2.9267(4)$ & - & & [150] \\
\hline$\left[\mathrm{AlMe}_{3}\left(\mathrm{Sb}^{t} \mathrm{Bu}_{3}\right)\right]$ & $2.8344(10)$ & - & & [150] \\
\hline$\left[\mathrm{AlEt}_{3}\left(\mathrm{Sb}^{t} \mathrm{Bu}_{3}\right)\right]$ & $2.8730(9)$ & - & & [150] \\
\hline$\left[\mathrm{Al}^{t} \mathrm{Bu}_{3}\left(\mathrm{SbMe}_{3}\right)\right]$ & $2.8431(6)$ & - & & [151] \\
\hline$\left[\mathrm{Al}^{t} \mathrm{Bu}_{3}\left(\mathrm{Sb}^{i} \mathrm{Bu}_{3}\right)\right]$ & $2.903(2)$ & - & & [155] \\
\hline$\left[\mathrm{AlEt}_{3}\left\{\mathrm{Sb}\left(\mathrm{SiMe}_{3}\right)_{3}\right\}\right]$ & $2.841(1)$ & & & [152] \\
\hline$\left[\mathrm{AlEt}_{3}\left\{\mathrm{Bi}\left(\mathrm{SiMe}_{3}\right)_{3}\right\}\right]$ & $2.921(2)$ & - & & [153] \\
\hline$\left[\mathrm{Al}^{t} \mathrm{Bu}_{3}\left(\mathrm{Bi}^{\mathrm{i}} \mathrm{Pr}_{3}\right)\right]$ & $3.088(1)$ & - & & [153] \\
\hline$\left[\mathrm{Al}^{t} \mathrm{Bu}_{3}\left(\mathrm{BiEt}_{3}\right)\right]$ & $2.940(1)$ & - & & [154] \\
\hline$\left[\mathrm{Al}^{t} \mathrm{Bu}_{2} \mathrm{Cl}\left\{\mathrm{Sb}\left(\mathrm{SiMe}_{3}\right)_{3}\right\}\right]$ & $2.82(1), 2.798(1)$ & $2.182(1), 2.183(1)$ & & [152] \\
\hline$\left[\mathrm{AlEtCl}_{2}\left\{\mathrm{P}\left(\mathrm{SiMe}_{3}\right)_{3}\right\}\right]$ & $2.435(3)$ & $2.144(4), 2.156(4)$ & & [156] \\
\hline$\left[\mathrm{Al}^{i} \mathrm{Bu}_{2} \mathrm{Cl}\left\{\mathrm{P}\left(\mathrm{SiMe}_{3}\right)_{3}\right\}\right]$ & $2.504(3)$ & $2.1794(4)$ & & [156] \\
\hline$\left[\mathrm{Al}^{i} \mathrm{Bu}_{2} \mathrm{Cl}\left\{\mathrm{As}\left(\mathrm{SiMe}_{3}\right)_{3}\right\}\right]$ & $2.573(1)$ & $2.176(2)$ & & [157] \\
\hline$\left[\mathrm{AlMe}_{2}(\mathrm{SePh})\left(\mathrm{PPh}_{3}\right)\right]$ & $2.517(1)$ & - & & [160] \\
\hline$\left[\mathrm{Al}\left(\mathrm{Ph}_{2} \mathrm{PCH}_{2} \mathrm{C}_{6} \mathrm{H}_{4}\right)_{3}\right]$ & $2.676(2), 2.782(2)$ & - & & {$[161,162]$} \\
\hline$\left[\mathrm{AlMe}_{2}\left(2,6-{ }^{t} \mathrm{Bu}_{2}-4-\mathrm{MeC}_{6} \mathrm{H}_{2} \mathrm{O}\right)\left(\mathrm{PMe}_{3}\right)\right]$ & $2.499(3)$ & - & & {$[163]$} \\
\hline$\left[\mathrm{AlMe}_{2}\left\{\mathrm{Ph}_{2} \mathrm{PC}_{6} \mathrm{H}_{2}(\mathrm{Me})\left({ }^{t} \mathrm{Bu}\right) \mathrm{O}\right\}\right]$ & $2.469(1)$ & - & 4-Coord $\mathrm{POC}_{2}$ & [164] \\
\hline$\left[\right.$ AlMe $\left.\left\{\mathrm{Ph}_{2} \mathrm{PC}_{6} \mathrm{H}_{2}(\mathrm{Me})(\mathrm{tBu}) \mathrm{O}\right\}_{2}\right]$ & $2.705(1), 2.607(1)$ & - & 5-Coord $\mathrm{P}_{2} \mathrm{O}_{2} \mathrm{C}$ & [164] \\
\hline$\left[\mathrm{Al}\left\{\mathrm{Ph}_{2} \mathrm{PC}_{6} \mathrm{H}_{2}(\mathrm{Me})(\mathrm{tBu}) \mathrm{O}\right\}_{3}\right]$ & $2.647(2), 2.714(2), 2.659(3)$ & - & 6-Coord $\mathrm{P}_{3} \mathrm{O}_{3}$ & [164] \\
\hline$\left[\mathrm{AlCl}_{2}(\mathrm{II})\right]^{\mathrm{a}}$ & $2.542(7)$ & - & TBP axial $\mathrm{P}$ & {$[166]$} \\
\hline$\left[\mathrm{Al}\left(\mathrm{CH}_{2} \mathrm{Ph}\right)_{2}(\mathrm{II})\right]^{\mathrm{a}}$ & $2.453(3)$ & - & 4-Coord $\mathrm{C}_{2} \mathrm{PN}$ & [166] \\
\hline$\left[\mathrm{AlCl}_{2}(\mathrm{II})\left(\mathrm{AlCl}_{3}\right)\right]^{\mathrm{a}}$ & $2.403(2), 2.412(2)$ & - & 4-Coord $\mathrm{PNCl}_{2}+4$-Coord $\mathrm{PCl}_{3}$ & [166] \\
\hline$\left[\mathrm{AlCl}_{2}(\mathbf{I})\right]^{\mathrm{a}}$ & $2.4884(8)$ & $2.1721(12)$ & 5-Coord $\mathrm{P}_{2} \mathrm{NCl}_{2}$ & [167] \\
\hline$\left[\mathrm{AlH}\left\{\operatorname{syn}-\left(\mathrm{P}_{2} \mathrm{~N}_{2}\right)\right\}\right]^{\mathrm{b}}$ & $2.517(3), 2.523(3)$ & & 5-Coord $\mathrm{P}_{2} \mathrm{~N}_{2} \mathrm{H}$ & [169] \\
\hline
\end{tabular}

a Anionic PNP pincer ligands.

b Macrocyclic bis(amidophosphine) ligand. 


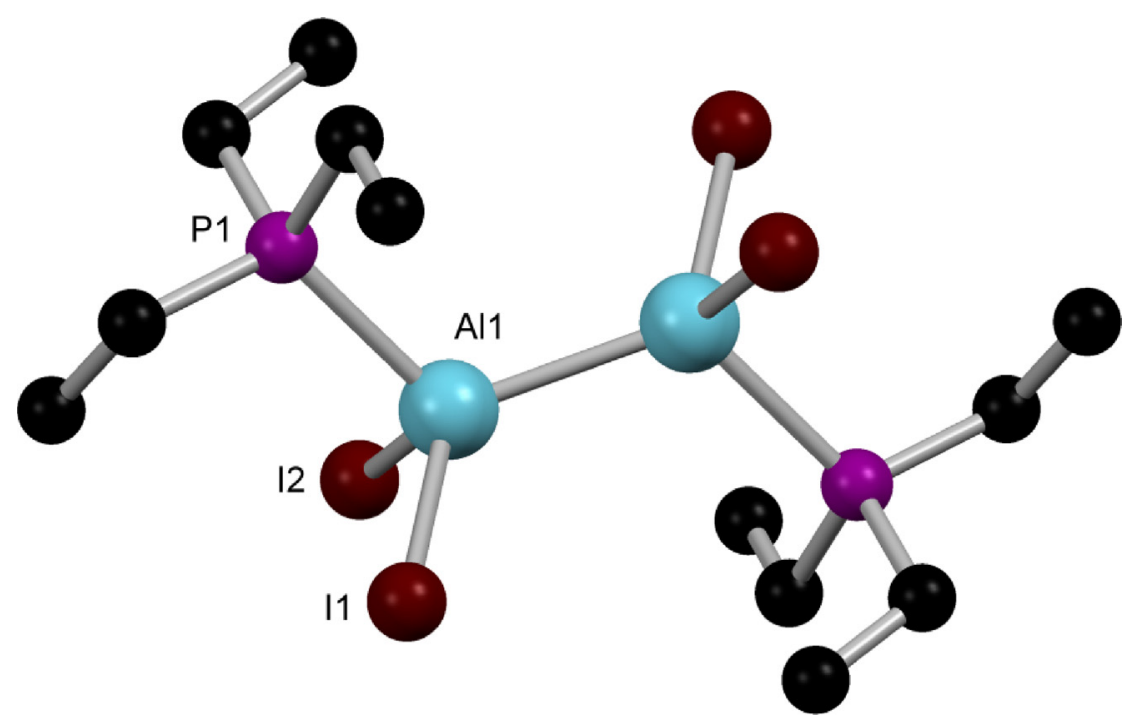

Fig. 18. View of the structure of $\left[\mathrm{Al}_{2} \mathrm{I}_{4}\left(\mathrm{PEt}_{3}\right)_{2}\right]$ redrawn from Ref. [133].

with the phosphine hydrochlorides, or addition of $\mathrm{HCl} / \mathrm{Et}_{2} \mathrm{O}$ to mixtures of $\mathrm{LiAlH}_{4}$ and the phosphine, produced $\left[\mathrm{AlH}_{3}\left(\mathrm{PCy}_{3}\right)\right]$, $\left[\mathrm{AlH}_{3}\left(\mathrm{P}^{t} \mathrm{Bu}_{3}\right)\right]$, and $\left[\left(\mathrm{AlH}_{3}\right)_{2}\left\{\mu-\mathrm{Cy}_{2} \mathrm{P}\left(\mathrm{CH}_{2}\right)_{2} \mathrm{PCy}\right\}\right]$ containing four-coordinate aluminium. These complexes are thermally stable to $>150^{\circ} \mathrm{C}$, their stability being attributed to steric hindrance preventing the formation of hydride bridges which is the decomposition route for small phosphine adducts. Attempts to form adducts with $\mathrm{Ph}_{2} \mathrm{P}\left(\mathrm{CH}_{2}\right)_{n} \mathrm{PPh}_{2} \quad(n=1$ or 2$)$ or $\mathrm{PhP}\left(\mathrm{CH}_{2} \mathrm{CH}_{2} \mathrm{PPh}_{2}\right)_{2}$ were unsuccessful, but with ${ }^{i} \mathrm{Pr}_{2} \mathrm{P}\left(\mathrm{CH}_{2}\right)_{2} \mathrm{P}^{i} \mathrm{Pr}_{2}$ both the four-coordinate dimer $\left[\left(\mathrm{AlH}_{3}\right)_{2}\left\{\mu-{ }^{i} \mathrm{Pr}_{2} \mathrm{P}\left(\mathrm{CH}_{2}\right)_{2} \mathrm{P}^{i} \mathrm{Pr}_{2}\right\}\right]$ (Fig. 19) and the five-coordinate polymer $\left[\left\{\left(\mathrm{AlH}_{3}\right)_{2}\{\mu\right.\right.$ $\left.\left.{ }^{i} \mathrm{Pr}_{2} \mathrm{P}\left(\mathrm{CH}_{2}\right)_{2} \mathrm{P}^{i} \mathrm{Pr}_{2}\right\}\right\}_{n}$ ] were isolated and structurally characterised. The Al-P bonds are long, in some cases marginally longer than the $\mathrm{Ga}-\mathrm{P}$ distance in gallium analogues (see below), and the ${ }^{31} \mathrm{P}\left\{{ }^{1} \mathrm{H}\right\}$ NMR coordination shifts are small, both suggesting weak Al-P bonds.

A large number of adducts of aluminium alkyls with phosphine ligands have been prepared; all appear to contain four-coordinate aluminium and are usually very moisture- and air-sensitive, although much less pyrophoric than the parent aluminium alkyls. In contrast to the aluminium halide complexes, there are di-, tri- (Fig. 20) and tetra-phosphine adducts of aluminium alkyls, although again all contain only one phosphorus bound to each aluminium [139-147]. Usually the $\mathrm{AlR}_{3}{ }_{3}$ and the phosphine are simply mixed in an inert solvent, but other routes have been described, for example, $\mathrm{Al}^{i} \mathrm{Bu}_{2} \mathrm{H}$ and the diphosphine dioxide, $\mathrm{Ph}_{2} \mathrm{P}(\mathrm{O}) \mathrm{CH}_{2} \mathrm{CH}_{2} \mathrm{P}(\mathrm{O}) \mathrm{Ph}_{2}$, produced $\left[\left(\mathrm{Al}^{i} \mathrm{Bu}_{3}\right)_{2}(\mu\right.$ $\left.\mathrm{Ph}_{2} \mathrm{PCH}_{2} \mathrm{CH}_{2} \mathrm{PPh}_{2}\right)$ ] by a sequence of reduction and rearrangement reactions [144]. $\left[\mathrm{Al}_{2} \mathrm{Me}_{3} \mathrm{Cl}_{3}\right]$ and $\mathrm{Ph}_{2} \mathrm{PCH}_{2} \mathrm{PPh}_{2}$ gave the scrambled product $\left[\left(\mathrm{AlCl}_{2} \mathrm{Me}\right)\left(\mathrm{AlCl}_{3}\right)\left(\mu-\mathrm{Ph}_{2} \mathrm{PCH}_{2} \mathrm{PPh}_{2}\right)\right][145]$.

For $\left[\mathrm{AlMe}_{3}\left(\mathrm{PR}_{3}\right)\right]\left(\mathrm{R}_{3}=\mathrm{Me}_{3}, \mathrm{Et}_{3}, \mathrm{Ph}_{3}, \mathrm{Me}_{2} \mathrm{Ph}, \mathrm{MePh}_{2}\right.$, $\left(\mathrm{CH}_{2} \mathrm{CH}_{2} \mathrm{CN}\right)_{3}, \mathrm{Cy}_{3},{ }^{t} \mathrm{Bu}_{3}$, (o-tolyl $)_{3}$ etc.) the coordination shifts $(\Delta)$ in the ${ }^{31} \mathrm{P}$ NMR spectra are positive for small phosphines, but become negative as the steric bulk increases, attributed to an increase in the repulsive interaction of the phosphine substituents with the methyl groups on the small aluminium centre [139]. The corresponding Al-Me ${ }^{13} \mathrm{C}\left\{{ }^{1} \mathrm{H}\right\}$ NMR shifts move to high frequency with increasing steric bulk of the phosphine. Consideration of the

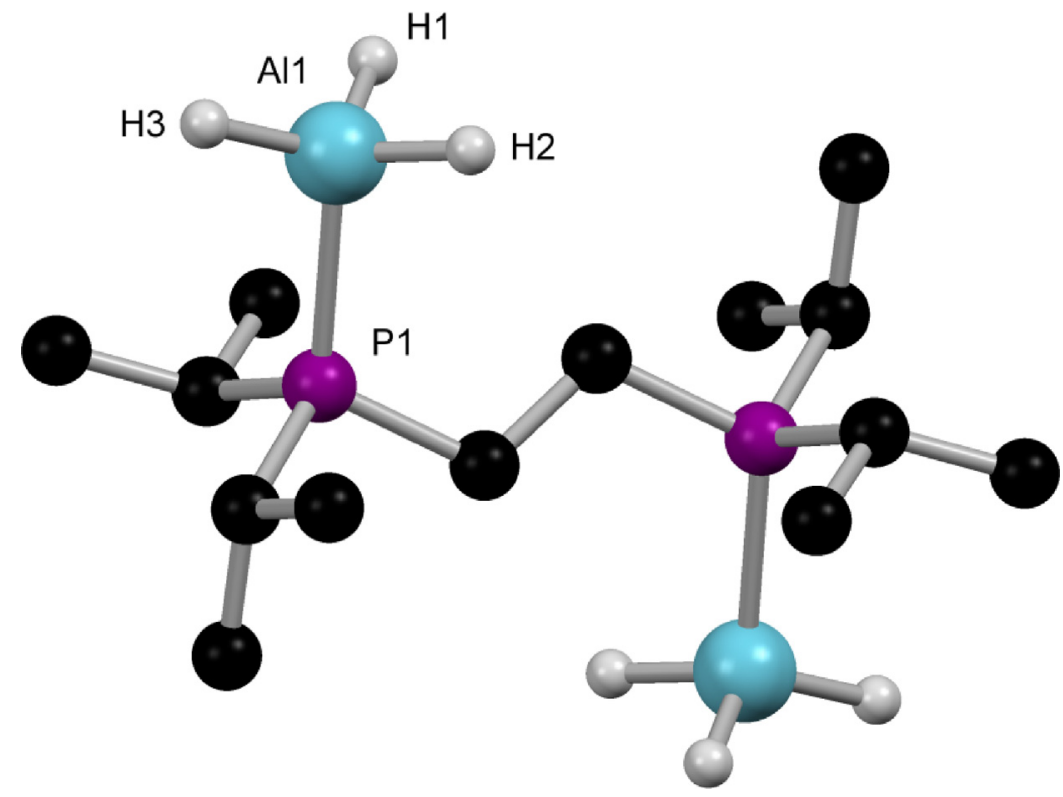

Fig. 19. View of the structure of $\left[\left(\mathrm{AlH}_{3}\right)_{2}\left\{\mu-{ }^{i} \mathrm{Pr}_{2} \mathrm{P}\left(\mathrm{CH}_{2}\right)_{2} \mathrm{P}^{i} \mathrm{Pr}_{2}\right\}\right]$ redrawn from Ref. [137]. 


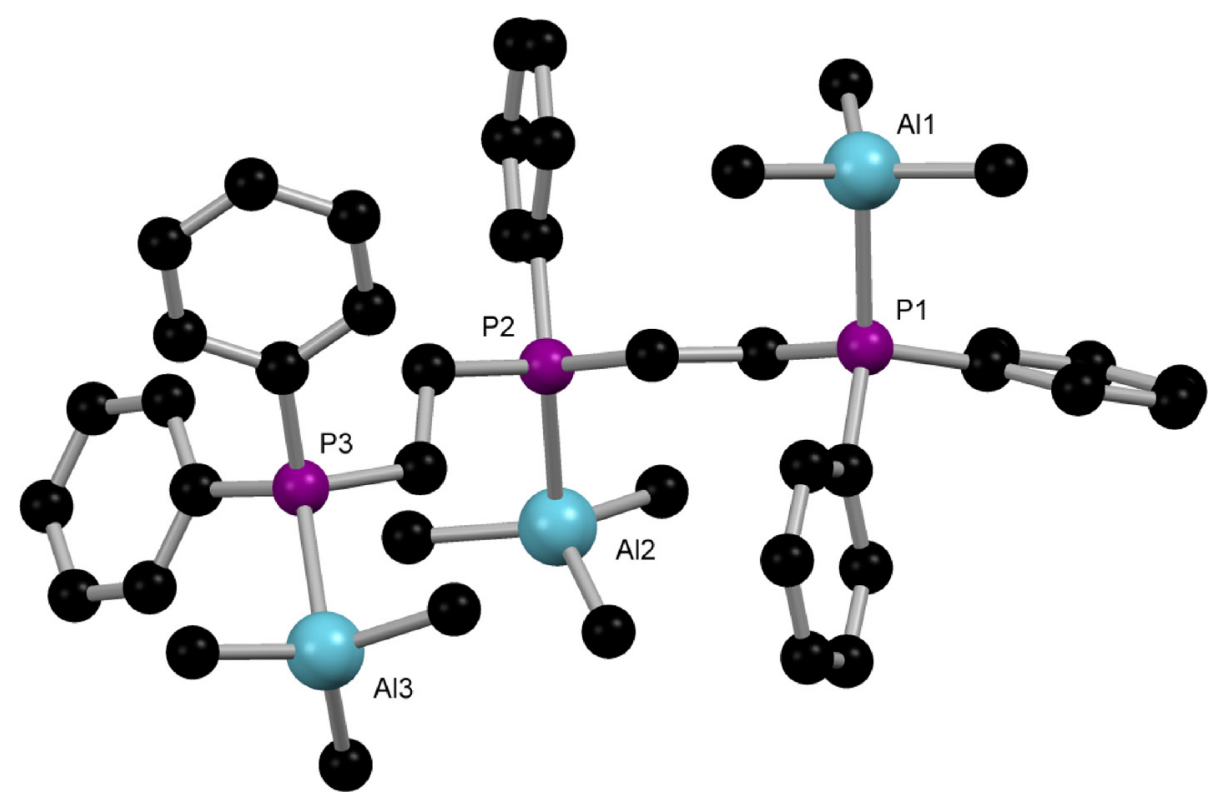

Fig. 20. View of the structure of $\left[\left(\mathrm{AlMe}_{3}\right)_{3}\left\{\mu-\left(\mathrm{Ph}_{2} \mathrm{PCH}_{2} \mathrm{CH}_{2}\right)_{2} \mathrm{PPh}\right\}\right]$ redrawn from Ref. [146].

structural data in Table 6 shows that longer Al-P and Al-As bonds are found in complexes with bulky $\mathrm{R}$ groups either on $\mathrm{Al}$ or $\mathrm{P} / \mathrm{As}$ (or both), showing clearly that steric effects are very important, although the detailed trends are irregular; the various contributing factors are discussed at length in [147].

Unusually for the elements covered in the article, a range of stibine and bismuthine complexes has been characterized. The work has been reviewed by Schulz [149] who also discusses related $\mathrm{R}_{2} \mathrm{Sb}^{-}$compounds and the use of such complexes in MOCVD of AlSb nanomaterials. Early work established that $\mathrm{SbR}_{3}$ ligands are chlorinated by $\mathrm{AlCl}_{3}$ [7] and there seem to be no reports of

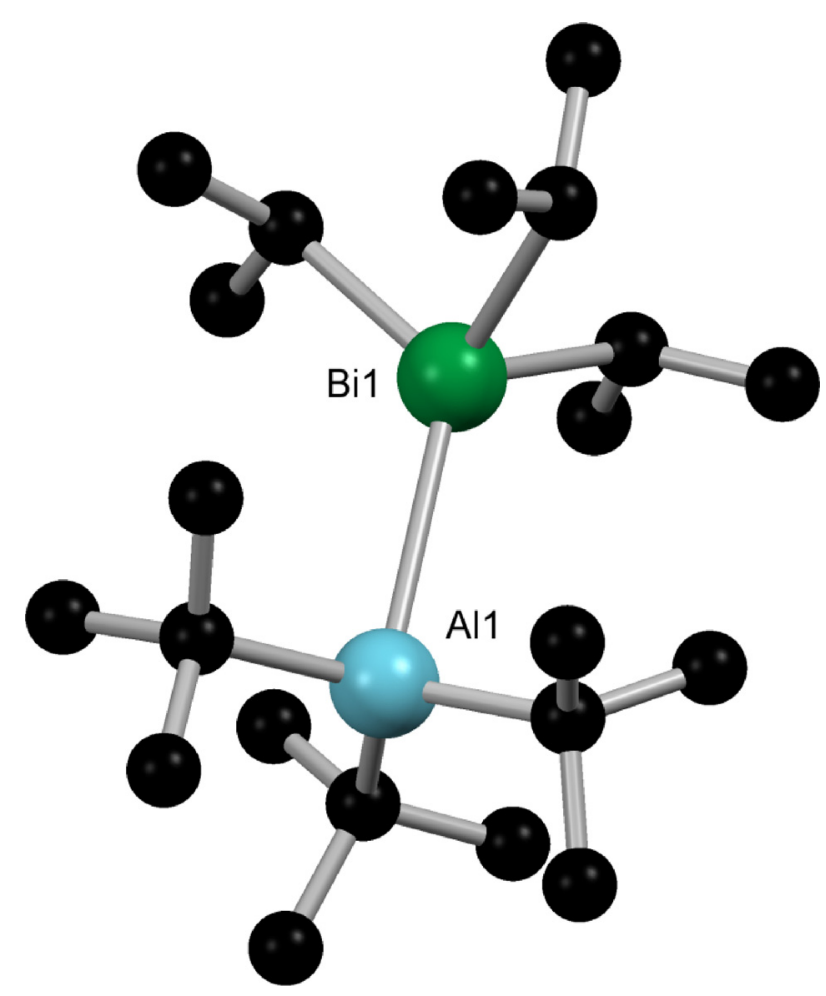

Fig. 21. View of the structure of $\left[\mathrm{Al}^{t} \mathrm{Bu}_{3}\left(\mathrm{Bi}^{i} \mathrm{Pr}_{3}\right)\right]$ redrawn from Ref. [153]. successful isolation of halide adducts. Mixing equimolar amounts of $\mathrm{AlR}_{3}{ }_{3}\left(\mathrm{R}^{\prime}=\mathrm{Me}, \mathrm{Et},{ }^{t} \mathrm{Bu}\right)$ with $\mathrm{SbR}_{3}\left(\mathrm{R}=\mathrm{Et},{ }^{n} \mathrm{Pr},{ }^{i} \mathrm{Pr},{ }^{s} \mathrm{Bu}\right.$ or $\left.{ }^{t} \mathrm{Bu}\right)$ produced colourless solid or liquid adducts $\left[\mathrm{AlR}_{3}{ }_{3}\left(\mathrm{SbR}_{3}\right)\right]$ which are very air-sensitive, and ${ }^{1} \mathrm{H}$ NMR studies in the presence of added stibine show fast exchange even at low temperatures [150]. Adducts of the silylstibine $\mathrm{Sb}\left(\mathrm{SiMe}_{3}\right)_{3}$ of type $\left[\mathrm{AlR}_{3}^{\prime}\left\{\mathrm{Sb}\left(\mathrm{SiMe}_{3}\right)_{3}\right\}\right]\left(\mathrm{R}^{\prime}=\mathrm{Me}, \mathrm{Et}\right.$, ${ }^{t} \mathrm{Bu}$ ) are formed in quantitative yields by combining the components in the absence of a solvent [152]. The bismuthine adducts $\left[\mathrm{AlR}_{3}^{\prime}\left(\mathrm{BiR}_{3}\right)\right]\left(\mathrm{R}^{\prime}=\mathrm{Me}, \mathrm{Et},{ }^{t} \mathrm{Bu} ; \mathrm{R}={ }^{i} \mathrm{Pr}\right.$ or $\left.\mathrm{SiMe}_{3}\right)$ (Fig. 21) were formed similarly and are extremely sensitive compounds; only the $\mathrm{Al}^{t} \mathrm{Bu}_{3}$ adducts persist in solution, whereas the others appear to completely dissociate into their constituents [153]. Chlorodialkylaluminiums, $\mathrm{R}_{2}{ }_{2} \mathrm{AlCl}\left(\mathrm{R}^{\prime}=\mathrm{Et},{ }^{i} \mathrm{Bu}\right)$ also form simple $1: 1$ adducts with $\mathrm{P}\left(\mathrm{SiMe}_{3}\right)_{3}, \mathrm{As}\left(\mathrm{SiMe}_{3}\right)_{3}$ and $\mathrm{Sb}\left(\mathrm{SiMe}_{3}\right)_{3}[152,156-158]$, but under other conditions dehalosilylation occurs giving four-membered ring systems $\left[\left\{\mathrm{R}_{2} \mathrm{AlE}\left(\mathrm{SiMe}_{2}\right)\right\}_{2}\right]$ (Fig. 22).

A variety of complexes with $\mathrm{R}_{2} \mathrm{P}(\mathrm{Y})-\mathrm{Al}$ linkages have been reported where $\mathrm{Y}$ is a charged ligand fragment containing anionic $\mathrm{C}-, \mathrm{N}-$ or O-donor groups. These include the complex of tris(o-(diphenylphosphinomethyl)phenyl)aluminium, $\left[\mathrm{Al}\left(\mathrm{Ph}_{2} \mathrm{PCH}_{2} \mathrm{C}_{6} \mathrm{H}_{4}\right)_{3}\right]$, made from $\mathrm{AlCl}_{3}$ and the aryllithium, which is a five-coordinate trigonal bipyramid $\left(\mathrm{P}_{2} \mathrm{C}_{3}\right)$ with axial $\mathrm{P}$ (Fig. 23) [161,162]. 2-Diphenylphosphinophenols form mono-, bis- and tris-adducts on reaction with $\mathrm{AlMe}_{3}$ in which the ligands chelate $(\mathrm{P}, \mathrm{O})$ as monoanions to the aluminium, with<smiles>[R][Si]([R])([R])[Si](C)(C)P([Si]([R])([R])[R])([Si](C)(C)C)([Si](C)(C)C)[Si](C)(C)C</smiles>

Fig. 22. Sketch of the dimer $\left[\left\{\mathrm{R}_{2} \mathrm{AlP}\left(\mathrm{SiMe}_{2}\right)\right\}_{2}\right]\left(\mathrm{R}=\mathrm{Et},{ }^{i} \mathrm{Bu}\right)$. 


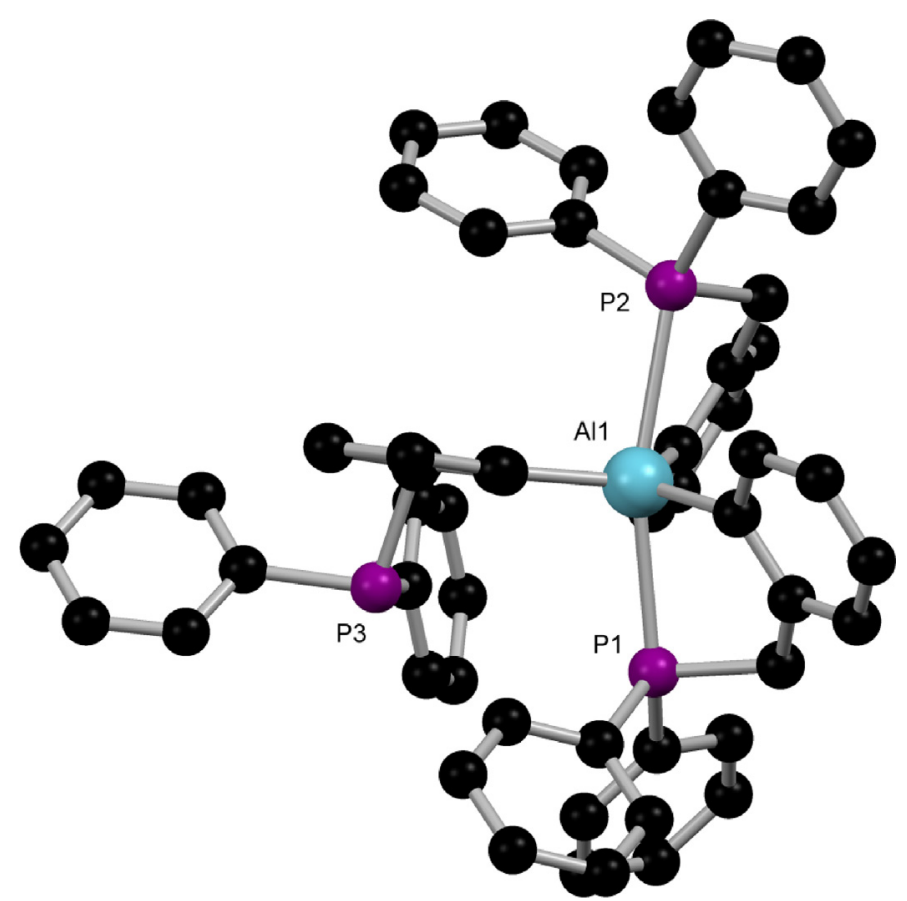

Fig. 23. View of the structure of $\left[\mathrm{Al}\left(\mathrm{Ph}_{2} \mathrm{PCH}_{2} \mathrm{C}_{6} \mathrm{H}_{4}\right)_{3}\right]$ redrawn from Ref. [161].

the Al-P distances increasing with increased coordination number as expected [164]. Chelating C,P coordination is found in closo-2-[(dimethylphosphino)methyl]-1,2-dicarbaborane (HCB) complexes, $\left[\mathrm{AlCl}_{2}(\mathbf{C B})\right]$ and $\left[\mathrm{AlCl}(\mathbf{C B})_{2}\right]$ [165]. Four- and fivecoordinate complexes with anionic amido-diphosphine (PNP) ligands (I) and (II) have been prepared [166,167].

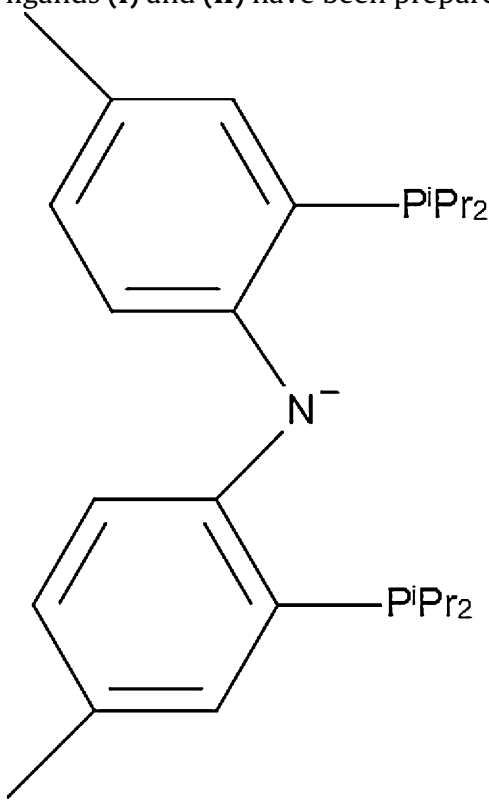

(I)

The macrocyclic bis(amidophosphine) ligand $\left(\mathbf{P}_{\mathbf{2}} \mathbf{N}_{\mathbf{2}}\right)$ is found in two forms syn and anti; the former produces five-coordinate $\left[\mathrm{AlCl}\left\{\right.\right.$ syn- $\left.\left.\left(\mathbf{P}_{\mathbf{2}} \mathbf{N}_{\mathbf{2}}\right)\right\}\right]\left(\mathrm{ClP}_{2} \mathrm{~N}_{2}\right.$ coordination), whereas the latter gives four-coordinate $\left[\mathrm{AlCl}\left\{\right.\right.$ anti- $\left.\left.\left(\mathbf{P}_{\mathbf{2}} \mathbf{N}_{\mathbf{2}}\right)\right\}\right]\left(\mathrm{ClPN}_{2}\right.$ coordination) [168]. In solution, NMR studies show anti $\rightarrow$ syn conversion via pyramidal inversion at phosphorus. $\mathrm{LiAlH}_{4}$ reduces $\left[\mathrm{AlCl}\left\{\right.\right.$ syn- $\left.\left.\left(\mathbf{P}_{\mathbf{2}} \mathbf{N}_{\mathbf{2}}\right)\right\}\right]$ to $\left[\mathrm{AlH}\left\{\right.\right.$ syn- $\left.\left.\left(\mathbf{P}_{\mathbf{2}} \mathbf{N}_{\mathbf{2}}\right)\right\}\right]$ [169].

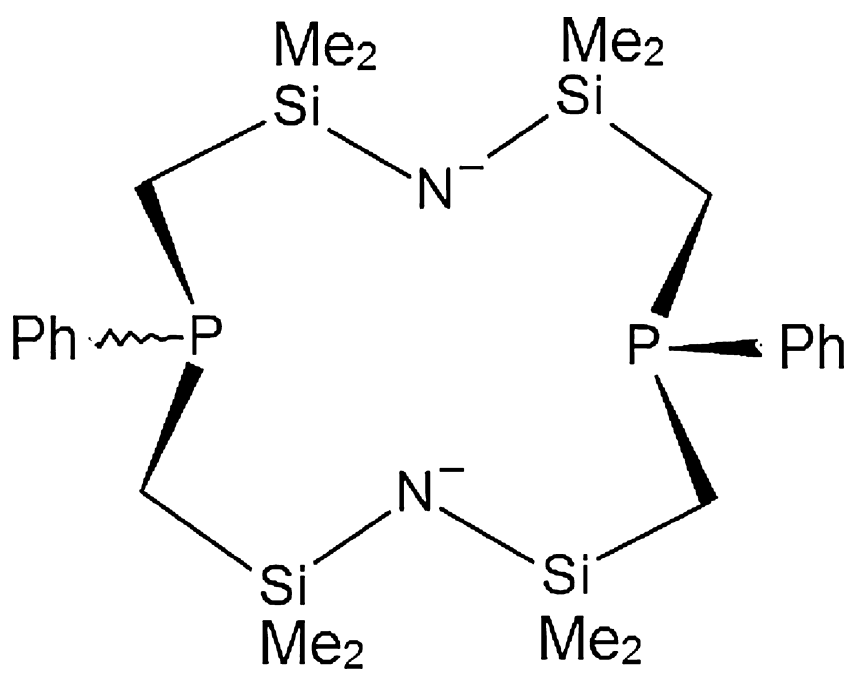

$\left(\mathbf{P}_{2} \mathbf{N}_{2}\right)$

\subsection{Gallium}

Currently the complexes of gallium(I) and gallium(II) are limited to a few examples, but a large number of gallium(III) complexes have been reported. Gallium(III) halide and alkyl complexes show close resemblance to those of aluminium, but significant differences to the indium complexes. Gallium(III) has essentially the same covalent radius as aluminium(III) (1.25 $)$, although the

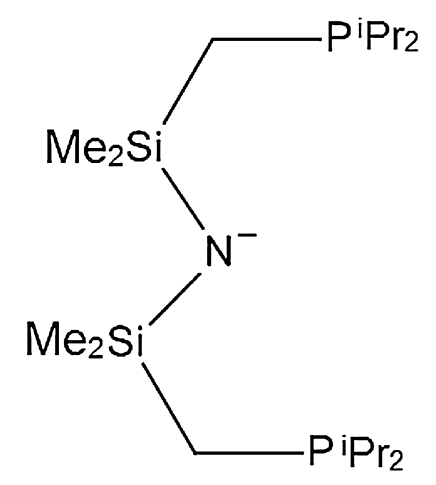

gallium compounds are often rather weaker Lewis acids. There is limited modelling work on the Lewis acidity of gallium systems, but it is likely that the key factors identified for boron and aluminium are also operating in the gallium systems. As discussed for aluminium (Section 6.2), other factors sometimes complicate interpretation of Lewis acidity based upon bond length data, but it seems 
Table 7

X-ray structures of gallium complexes.

\begin{tabular}{|c|c|c|c|c|}
\hline Complex & $\mathrm{Ga}-\mathrm{P} / \mathrm{As} / \mathrm{Sb}(\AA)$ & $\mathrm{Ga}-\mathrm{X}(\AA)^{\mathrm{a}}$ & Comments & Ref. \\
\hline$\left[\mathrm{Ga}_{8} \mathrm{I}_{8}\left(\mathrm{PEt}_{3}\right)_{6}\right]$ & $2.412(3)-2.428(2)$ & $2.6483(9)-2.6757(10)$ & $\mathrm{Ga}_{8}$ ring & [183] \\
\hline$\left[\mathrm{Ga}\left(\mathrm{PPh}_{3}\right)_{3}\right]\left[\mathrm{Al}\left(\left\{\mathrm{OC}\left(\mathrm{CF}_{3}\right)_{3}\right\}_{4}\right]\right.$ & $2.65-2.76$ & & & {$[184]$} \\
\hline$\left[\mathrm{Ga}\left(\mathrm{P}^{\mathrm{t}} \mathrm{Bu}_{3}\right)_{2}\right]\left[\mathrm{Al}\left(\left\{\mathrm{OC}\left(\mathrm{CF}_{3}\right)_{3}\right\}_{4}\right]\right.$ & $2.774(3)$ & & & {$[185]$} \\
\hline$\left[\mathrm{Ga}_{2} \mathrm{Cl}_{4}\left(\mathrm{PEt}_{3}\right)_{2}\right]$ & $2.4269(5)$ & $2.2248(6), 2.2336(6)$ & Ga-Ga dimer & {$[172]$} \\
\hline$\left[\mathrm{Ga}_{2} \mathrm{Br}_{4}\left(\mathrm{PEt}_{3}\right)_{2}\right]$ & $2.400(2)$ & $2.427(1), 2.368(1)$ & Ga-Ga dimer & {$[172]$} \\
\hline$\left[\mathrm{Ga}_{2} \mathrm{I}_{4}\left(\mathrm{PEt}_{3}\right)_{2}\right]$ & $2.413(3)$ & $2.584(1), 2.591(1)$ & Ga-Ga dimer & {$[186]$} \\
\hline$\left[\mathrm{Ga}_{2} \mathrm{I}_{4}\left(\mathrm{PPh}_{3}\right)_{2}\right]$ & $2.445(3)$ & $2.555(1), 2.582(1)$ & Ga-Ga dimer & {$[186]$} \\
\hline$\left[\mathrm{Ga}_{2} \mathrm{I}_{4}\left(\mathrm{PHCy}_{2}\right)_{2}\right]$ & $2.424(2)$ & $2.5924(10), 2.5755(11)$ & Ga-Ga dimer & [188] \\
\hline$\left[\mathrm{Ga}_{2} \mathrm{I}_{4}\left(\mathrm{PH}^{\mathrm{t}} \mathrm{Bu}_{2}\right)_{2}\right]$ & $2.4459(10)$ & $2.5935(6), 2.5745(6)$ & Ga-Ga dimer & [188] \\
\hline$\left[\mathrm{Ga}_{2} \mathrm{Br}_{4}\left(\mathrm{PHCy}_{2}\right)_{2}\right]$ & $2.4164(7)$ & $2.3807(5), 2.3612(5)$ & Ga-Ga dimer & [189] \\
\hline$\left[\mathrm{Ga}_{2} \mathrm{I}_{4}\left(\mathrm{AsEt}_{3}\right)_{2}\right]$ & $2.485(5)$ & $2.564(4), 2.568(4)$ & Ga-Ga dimer & [187] \\
\hline$\left.\left[\mathrm{Ga}_{3} \mathrm{I}_{5}\left(\mathrm{PEt}_{3}\right)_{3}\right)\right]$ & $2.404(3), 2.427(3)$ & $2.601(1)-2.627(1)$ & & {$[186]$} \\
\hline$\left[\mathrm{GaCl}_{3}\left(\mathrm{PPh}_{3}\right)\right]$ & $2.3717(16)$ & $2.1677(15)-2.1696(15)$ & & [171] \\
\hline$\left[\mathrm{GaBr}_{3}\left(\mathrm{PPh}_{3}\right)\right]$ & $2.3848(13), 2.3879(13)$ & $2.3048(7)-2.3225(7)$ & & {$[171]$} \\
\hline$\left[\mathrm{GaI}_{3}\left(\mathrm{PPh}_{3}\right)\right]$ & $2.413(4) / 2.416(5)$ & $2.518(2) / 2.5212(9)$ & two independent studies & {$[173,176]$} \\
\hline$\left[\mathrm{GaCl}_{3}\left(\mathrm{PEt}_{3}\right)\right]$ & $2.3531(5)$ & $2.1746(5)-2.1836(5)$ & & {$[172]$} \\
\hline$\left[\mathrm{GaCl}_{3}\left(\mathrm{PMe}_{3}\right)\right]$ & $2.353(2)$ & $2.171(1), 2.176(1)$ & & {$[174]$} \\
\hline$\left[\mathrm{GaBr}_{3}\left\{\mathrm{P}\left(p-\mathrm{C}_{6} \mathrm{H}_{4} \mathrm{OMe}\right)_{3}\right\}\right]$ & $2.500(2), 2.494(2)$ & $2.3166(6)-2.319(3)$ & & [177] \\
\hline$\left[\mathrm{GaI}_{3}\left\{\mathrm{P}\left(p-\mathrm{C}_{6} \mathrm{H}_{4} \mathrm{OMe}\right)_{3}\right\}\right]$ & $2.400(1)$ & $2.5257(5)-2.5408(6)$ & & [177] \\
\hline$\left[\mathrm{GaBr}_{3}\left(\mathrm{PH}^{t} \mathrm{Bu}_{2}\right)\right]$ & $2.411(2), 2.413(2)$ & $2.330(1), 2.332(1)$ & & {$[122]$} \\
\hline$\left[\mathrm{GaCl}_{3}\left\{\mathrm{P}\left(\mathrm{SiMe}_{3}\right)_{3}\right\}\right]$ & $2.379(5), 2.380(5)$ & $2.169(4), 2.176(4)$ & & {$[178]$} \\
\hline$\left[\mathrm{GaBr}_{3}\left\{\mathrm{P}\left(\mathrm{SiMe}_{3}\right)_{3}\right\}\right]$ & $2.362(4)$ & $2.315(2)$ & & {$[178]$} \\
\hline$\left[\mathrm{GaI}_{3}\left\{\mathrm{P}\left(\mathrm{SiMe}_{3}\right)_{3}\right\}\right]$ & $2.347(4)$ & $2.564(5)$ & & {$[178]$} \\
\hline$\left[\mathrm{GaCl}_{3}\left(\mathrm{AsPh}_{3}\right)\right]$ & $2.449(6), 2.540(6)$ & $2.146(2)-2.164(2)$ & & {$[124]$} \\
\hline$\left[\mathrm{GaI}_{3}\left(\mathrm{AsPh}_{3}\right)\right]$ & $2.490(1)$ & $2.509(1)$ & & {$[173,175]$} \\
\hline$\left[\mathrm{GaCl}_{3}\left(\mathrm{AsMe}_{3}\right)\right]$ & $2.4332(12)$ & $2.1709(15)-2.1768(12)$ & & {$[170]$} \\
\hline$\left[\mathrm{GaBr}_{3}\left(\mathrm{AsMe}_{3}\right)\right]$ & $2.438(2)$ & $2.3154(19)-2.3226(15)$ & & {$[170]$} \\
\hline$\left[\mathrm{GaI}_{3}\left(\mathrm{AsMe}_{3}\right)\right]$ & $2.4593(13)$ & $2.5300(11)-2.5344(9)$ & & {$[170]$} \\
\hline$\left[\mathrm{GaI}_{3}\left\{\mathrm{As}\left(p-\mathrm{MeOC}_{6} \mathrm{H}_{4}\right)_{3}\right\}\right]$ & $2.509(3)$ & $2.505(1)$ & & [187] \\
\hline$\left[\mathrm{GaCl}_{2}\left\{0-\mathrm{C}_{6} \mathrm{H}_{4}\left(\mathrm{PMe}_{2}\right)_{2}\right\}_{2}\right]\left[\mathrm{GaCl}_{4}\right]$ & $2.4794(6), 2.4806(5)$ & $2.3585(5)$ & & {$[170]$} \\
\hline$\left[\mathrm{GaBr}_{2}\left\{0-\mathrm{C}_{6} \mathrm{H}_{4}\left(\mathrm{PMe}_{2}\right)_{2}\right\}_{2}\right]\left[\mathrm{GaBr}_{4}\right]$ & $2.4875(12), 2.4751(12)$ & $2.5276(6)$ & & {$[170]$} \\
\hline$\left[\mathrm{GaI}_{2}\left\{0-\mathrm{C}_{6} \mathrm{H}_{4}\left(\mathrm{PMe}_{2}\right)_{2}\right\}_{2}\right]\left[\mathrm{GaI}_{4}\right]$ & $2.487(2), 2.499(2)$ & $2.7481(6)$ & & {$[170]$} \\
\hline$\left[\mathrm{GaCl}_{2}\left\{0-\mathrm{C}_{6} \mathrm{H}_{4}\left(\mathrm{PPh}_{2}\right)_{2}\right\}\right]\left[\mathrm{GaCl}_{4}\right]$ & $2.3787(12), 2.3865(11)$ & $2.1457(11), 2.1575(12)$ & & {$[170]$} \\
\hline$\left[\mathrm{GaI}_{2}\left\{0-\mathrm{C}_{6} \mathrm{H}_{4}\left(\mathrm{PPh}_{2}\right)_{2}\right\}\right]\left[\mathrm{GaI}_{4}\right]$ & $2.3976(13), 2.4085(12)$ & $2.4904(6), 2.4912(7)$ & & {$[179]$} \\
\hline$\left[\left\{\mathrm{GaBr}_{3}\right\}_{2}\left\{\mu-\mathrm{Et}_{2} \mathrm{P}\left(\mathrm{CH}_{2}\right)_{2} \mathrm{PEt}_{2}\right\}\right]$ & $2.3614(13)$ & $2.3033(8)-2.3246(8)$ & & {$[170]$} \\
\hline$\left[\left\{\mathrm{GaI}_{3}\right\}_{2}\left\{\mu-\mathrm{Et}_{2} \mathrm{P}\left(\mathrm{CH}_{2}\right)_{2} \mathrm{PEt}_{2}\right\}\right]$ & $2.3769(15)$ & $2.5200(10)-2.5418(9)$ & & {$[170]$} \\
\hline$\left[\left\{\mathrm{GaCl}_{3}\right\}_{2}\left\{\mu-\mathrm{Ph}_{2} \mathrm{P}\left(\mathrm{CH}_{2}\right)_{2} \mathrm{PPh}_{2}\right\}\right]$ & $2.3854(8)$ & $2.1608(8)-2.1648(8)$ & & {$[180]$} \\
\hline$\left[\left\{\mathrm{GaI}_{3}\right\}_{2}\left\{\mu-\mathrm{Ph}_{2} \mathrm{P}\left(\mathrm{CH}_{2}\right)_{2} \mathrm{PPh}_{2}\right\}\right]$ & $2.404(9), 2.410(9)$ & $2.481(5)-2.533(5)$ & & {$[176]$} \\
\hline$\left[\left\{\mathrm{GaBr}_{3}\right\}_{2}\left\{\mu-\mathrm{Ph}_{2} \mathrm{PCH}=\mathrm{CHPPh}_{2}\right\}\right]$ & $2.400(2)$ & $2.2936(6)-2.3125(5)$ & & [181] \\
\hline$\left[\left\{\mathrm{GaI}_{3}\right)_{2}\left\{\mu-\mathrm{o}-\mathrm{C}_{6} \mathrm{H}_{4}\left(\mathrm{CH}_{2} \mathrm{PPh}_{2}\right\}_{2}\right]\right.$ & $2.3872(9), 2.3891(9)$ & $2.5143(4)-2.5516(5)$ & & {$[171]$} \\
\hline$\left[\mathrm{GaCl}_{2}\left\{0-\mathrm{C}_{6} \mathrm{H}_{4}\left(\mathrm{AsMe}_{2}\right\}_{2}\right]\left[\mathrm{GaCl}_{4}\right]\right.$ & $2.442(1)$ & $2.123(3)$ & & {$[170]$} \\
\hline$\left[\mathrm{GaI}_{2}\left\{0-\mathrm{C}_{6} \mathrm{H}_{4}\left(\mathrm{AsMe}_{2}\right\}_{2}\right]\left[\mathrm{GaI}_{4}\right]\right.$ & $2.450(2)$ & $2.491(3)$ & & {$[170]$} \\
\hline$\left[\left\{\mathrm{GaI}_{3}\right\}_{2}\left\{\mu-\mathrm{Ph}_{2} \mathrm{As}\left(\mathrm{CH}_{2}\right)_{2} \mathrm{AsPh}_{2}\right\}\right]$ & $2.4875(10), 2.4885(10)$ & $2.4944(9)-2.5329(9)$ & & {$[170]$} \\
\hline$\left[\mathrm{GaI}_{2}\left\{\mathrm{PhP}\left(o-\mathrm{C}_{6} \mathrm{H}_{4}\left(\mathrm{PPh}_{2}\right)_{2}\right\}\right]\left[\mathrm{GaI}_{4}\right]\right.$ & $2.435(2), 2.420(2)$ & $2.5473(8), 2.5818(8)$ & & {$[179]$} \\
\hline$\left[\left(\mathrm{GaI}_{3}\right)_{3}\left\{\mathrm{MeC}\left(\mathrm{CH}_{2} \mathrm{AsMe}_{2}\right)_{3}\right\}\right]$ & $2.470(2)-2.488(2)$ & $2.511(2)-2.533(2)$ & & {$[182]$} \\
\hline$\left[\left(\mathrm{GaCl}_{3}\right)\left(\mathrm{GaCl}_{2}\right)\left\{\mathrm{MeC}\left(\mathrm{CH}_{2} \mathrm{AsMe}_{2}\right)_{3}\right\}\right]\left[\mathrm{GaCl}_{4}\right]$ & $2.435(1)-2.465(2)$ & $2.160(1)-2.164(3)$ & & {$[182]$} \\
\hline$\left[\mathrm{GaCl}_{2}\left\{\mathrm{MeC}\left(\mathrm{CH}_{2} \mathrm{AsMe}_{2}\right)_{3}\right\}\right]\left[\mathrm{GaCl}_{4}\right]$ & 2.4361(1), 2.444(1) & $2.210(7), 2.207(2)$ & & [182] \\
\hline$\left[\mathrm{GaH}_{3}\left(\mathrm{PMe}_{3}\right)\right]$ & $2.3857(6)$ & & Also electron diffraction & [207] \\
\hline$\left[\mathrm{GaH}_{3}\left(\mathrm{PCy}_{3}\right)\right]$ & $2.460(2)$ & & & [208] \\
\hline$\left[\mathrm{GaH}_{3}\left(\mathrm{P}^{t} \mathrm{Bu}_{3}\right)\right]$ & $2.446(2), 2.471(3)$ & & & [137] \\
\hline$\left[\left(\mathrm{GaH}_{3}\right)_{2}\left\{\mu-\mathrm{Me}_{2} \mathrm{P}\left(\mathrm{CH}_{2}\right)_{2} \mathrm{PMe}_{2}\right\}\right]$ & $2.399(4) / 2.403(1)$ & & Two independent studies & {$[208,209]$} \\
\hline$\left[\mathrm{GaH}_{2} \mathrm{Cl}\left(\mathrm{PCy}_{3}\right)\right]$ & $2.403(4)$ & $2.107(8)$ & & [210] \\
\hline$\left[\mathrm{GaH}_{2} \mathrm{Cl}\left(\mathrm{PMe}_{3}\right)\right]$ & $2.3797(18)$ & $2.237(2)$ & & [211] \\
\hline$\left[\mathrm{GaHCl}_{2}\left(\mathrm{PCy}_{3}\right)\right]$ & $2.432(1)$ & $2.182(2), 2.200(2)$ & & {$[212]$} \\
\hline$\left[\mathrm{GaHCl}_{2}\left(\mathrm{PPh}_{3}\right)\right]$ & $2.4038(6)$ & $2.1982(7), 2.1913(7)$ & & {$[212]$} \\
\hline$\left[\left(\mathrm{GaHCl}_{2}\right)_{2}\left(\mu-\mathrm{Ph}_{2} \mathrm{P}\left(\mathrm{CH}_{2}\right)_{2} \mathrm{PPh}_{2}\right)\right]$ & $2.426(1)$ & $2.1841(9), 2.2056(8)$ & & {$[212]$} \\
\hline$\left[\mathrm{GaMe}_{3}\left(\mathrm{PMe}_{3}\right)\right]$ & $2.455(4)$ & & & [199] \\
\hline$\left[\mathrm{GaMe}_{3}\left(\mathrm{PPh}_{3}\right)\right]$ & $2.5375(3)$ & & & {$[141]$} \\
\hline$\left[\mathrm{GaEt}_{3}\left(\mathrm{PPh}_{3}\right)\right]$ & $2.5375(5)$ & & & {$[141]$} \\
\hline$\left[\mathrm{GaPh}_{3}\left\{\mathrm{P}\left(\mathrm{SiMe}_{3}\right)_{3}\right\}\right]$ & $2.539(6)$ & & & [198] \\
\hline$\left[\mathrm{Ga}\left(\mathrm{C}_{6} \mathrm{~F}_{5}\right)_{3}\left(\mathrm{PH}_{2} \mathrm{Ph}\right)\right]$ & $2.477(1)$ & & & [203] \\
\hline$\left[\left(\mathrm{GaEt}_{3}\right)_{2}\left(\mu-\mathrm{Ph}_{2} \mathrm{P}\left(\mathrm{CH}_{2}\right)_{3} \mathrm{PPh}_{2}\right)\right]$ & $2.5238(5)$ & & & {$[141]$} \\
\hline$\left[\left(\mathrm{GaMe}_{3}\right)_{2}\left(\mu-\mathrm{Ph}_{2} \mathrm{P}\left(\mathrm{CH}_{2}\right)_{3} \mathrm{PPh}_{2}\right)\right]$ & $2.5069(4)$ & & & [141] \\
\hline$\left[\left(\mathrm{GaMe}_{3}\right)_{2}\left(\mu-\mathrm{Ph}_{2} \mathrm{P}\left(\mathrm{CH}_{2}\right)_{2} \mathrm{PPh}_{2}\right)\right]$ & $2.546(4)$ & & & {$[146]$} \\
\hline$\left[\left(\mathrm{GaMe}_{3}\right)_{2}\left(\mu-\mathrm{Ph}_{2} \mathrm{P}\left(\mathrm{CH}_{2}\right)_{5} \mathrm{PPh}_{2}\right)\right]$ & $2.496(2)$ & & & {$[141]$} \\
\hline$\left[\mathrm{GaEt}_{3}\left\{\mathrm{P}\left(\mathrm{SiMe}_{3}\right)_{3}\right\}\right]$ & $2.582(1)$ & & & [194] \\
\hline$\left[\mathrm{GaiButBu}_{2}\left\{\mathrm{P}^{i} \mathrm{Pr}_{3}\right)\right]$ & $2.720(1)$ & & & {$[194]$} \\
\hline$\left[\mathrm{Ga}\left(\mathrm{Me}_{3} \mathrm{CCH}_{2}\right)_{3}\left(\mathrm{PHPh}_{2}\right)\right]$ & $2.683(5)$ & & & [196] \\
\hline$\left[\mathrm{GaPh}_{3}\left\{\mathrm{As}\left(\mathrm{SiMe}_{3}\right)_{3}\right\}\right]$ & $2.571(1)$ & & & [201] \\
\hline$\left[\mathrm{Ga}^{t} \mathrm{Bu}_{3}\left(\mathrm{As}^{i} \mathrm{Pr}_{3}\right)\right]$ & $2.905(1)$ & & & {$[194]$} \\
\hline$\left[\mathrm{GaEt}_{3}\left\{\mathrm{As}\left(\mathrm{SiMe}_{3}\right)_{3}\right\}\right]$ & $2.684(2)$ & & & [194] \\
\hline$\left[\mathrm{GaEt}_{3}\left\{\mathrm{Sb}\left(\mathrm{SiMe}_{3}\right)_{3}\right\}\right]$ & $2.854(1)$ & & & {$[194]$} \\
\hline$\left[\mathrm{Ga}^{t} \mathrm{Bu}_{3}\left(\mathrm{SbMe}_{3}\right)\right]$ & $2.8435(3)$ & & & [191] \\
\hline$\left[\mathrm{GatBu}_{3}\left(\mathrm{SbEt}_{3}\right)\right]$ & $2.8479(5)$ & & & [192] \\
\hline$\left[\mathrm{Ga}^{t} \mathrm{Bu}_{3}\left(\mathrm{SbEt}_{2}{ }^{t} \mathrm{Bu}\right)\right]$ & $2.9243(5)$ & & & [193] \\
\hline$\left[\mathrm{GatBu}_{3}\left(\mathrm{Sb}^{i} \mathrm{Pr}_{3}\right)\right]$ & $2.9618(1)$ & & & [192] \\
\hline
\end{tabular}


Table 7 (Continued)

\begin{tabular}{|c|c|c|c|c|}
\hline Complex & $\mathrm{Ga}-\mathrm{P} / \mathrm{As} / \mathrm{Sb}(\AA)$ & $\mathrm{Ga}-\mathrm{X}(\AA)^{\mathrm{a}}$ & Comments & Ref. \\
\hline$\left[\mathrm{GaEt}_{3}\left\{\mathrm{Sb}\left(\mathrm{SiMe}_{3}\right)_{3}\right\}\right]$ & $2.846(5)$ & & & [190] \\
\hline$\left[\mathrm{Ga}^{t} \mathrm{Bu}_{3}\left(\mathrm{BiEt}_{3}\right)\right]$ & $2.966(1)$ & & & [154] \\
\hline$\left[\mathrm{GaMe}_{3}\left\{\mathrm{Bi}\left(\mathrm{SiMe}_{3}\right)_{3}\right\}\right]$ & $2.966(1)$ & & & {$[200]$} \\
\hline$\left[\mathrm{Ga}^{t} \mathrm{Bu}_{3}\left(\mathrm{Bi}^{i} \mathrm{Pr}_{3}\right)\right]$ & $3.135(1)$ & & & {$[200]$} \\
\hline$\left[\mathrm{GaPh}_{2} \mathrm{Cl}\left\{\mathrm{P}\left(\mathrm{SiMe}_{3}\right)_{3}\right\}\right]$ & $2.459(2)$ & $2.240(3)$ & & [198] \\
\hline$\left[\mathrm{Ga}\left(\mathrm{Me}_{3} \mathrm{CCH}_{2}\right)_{2} \mathrm{Cl}\left\{\mathrm{P}\left(\mathrm{SiMe}_{3}\right)_{3}\right\}\right]$ & $2.534(2)$ & $2.258(2)$ & & [197] \\
\hline$\left[\mathrm{Ga}\left(\mathrm{Me}_{3} \mathrm{CCH}_{2}\right)_{2} \mathrm{Cl}\left\{\mathrm{As}\left(\mathrm{SiMe}_{3}\right)_{3}\right\}\right]$ & $2.626(1)$ & $2.242(1)$ & & [197] \\
\hline$\left[\mathrm{GaMe}_{2} \mathrm{Cl}\left(\mathrm{Ph}_{2} \mathrm{PCH}_{2} \mathrm{PPh}_{2}\right)\right]$ & $2.535(2)$ & $2.260(2)$ & & {$[202]$} \\
\hline$\left[\mathrm{GaPhI}_{2}\left(\mathrm{SbPh}_{3}\right)\right]$ & $2.6898(8)$ & $2.5583(6), 2.5471(6)$ & & {$[204]$} \\
\hline$\left[\mathrm{Ga}\left(\mathrm{Ph}_{2} \mathrm{PCH}_{2} \mathrm{C}_{6} \mathrm{H}_{4}\right)_{3}\right]$ & $2.553(2)$ & & & {$[162]$} \\
\hline$\left[\mathrm{GaMe}_{2}\left\{\mathrm{C}_{5} \mathrm{H}_{4}\left(\mathrm{CH}_{2}\right)_{2} \mathrm{P}^{t} \mathrm{Bu}_{2}\right\}\right]$ & $2.493(2)$ & & & [215] \\
\hline$\left[\mathrm{GaMe}_{2}(\mathbf{C B})\right]^{\mathrm{b}}$ & $2.438(1)$ & & $\mathrm{C}_{3} \mathrm{P}$ coord. & [165] \\
\hline$\left[\mathrm{GaCl}(\mathbf{C B})_{2}\right]^{\mathrm{b}}$ & $2.651(2), 2.221(3)$ & & $\mathrm{C}_{2} \mathrm{P}_{2} \mathrm{Cl}$ coord & [165] \\
\hline$\left[\mathrm{GaCl}_{2}(\mathrm{III})\right]^{\mathrm{c}}$ & $2.512(2), 2.524(2)$ & Not quoted & $\mathrm{Cl}_{2} \mathrm{P}_{2} \mathrm{~N}$ coord & [217] \\
\hline$\left[\mathrm{GaCl}_{2}(\mathrm{III}-\mathrm{H})\right]\left[\mathrm{GaCl}_{4}\right]^{\mathrm{d}}$ & $2.3819(6), 2.378(6)$ & Not quoted & $\mathrm{Cl}_{2} \mathrm{P}_{2}$ coord & {$[217]$} \\
\hline$\left[\mathrm{GaBr}_{2}(\mathbf{I I I}-\mathbf{H})\right]\left[\mathrm{GaBr}_{4}\right]^{\mathrm{d}}$ & $2.384(2), 2.376(2)$ & Not quoted & $\mathrm{Br}_{2} \mathrm{P}_{2}$ coord & [217] \\
\hline
\end{tabular}

a Values refer to pnictogen coordinated fragment; data for $\left[\mathrm{GaX}_{4}\right]^{-}$are not given.

b HCB is the carbaborane (see text).

c III is the PNP pincer anion.

d III-H is the neutral PNP pincer.

clear in the case of gallium(III) that, apart from a few anomalies, the data indicate an order with halide $\mathrm{Cl}>\mathrm{Br}>\mathrm{I}[131,170,171]$. Analogous to the aluminium system, $\mathrm{GaF}_{3}$ is an inert polymer and no soft donor complexes are known [130]. For neutral phosphines and arsines, the gallium(III) overwhelmingly favours distorted tetrahedral four-coordination, and six-coordination is achieved only in some chelating diphosphine adducts. Five- and six-coordination does occur in some complexes of anionic hybrid ligands. A fair number of $\left[\mathrm{GaX}_{3}\left(\mathrm{ER}_{3}\right)\right]$ complexes were obtained pre-1975, although $\mathrm{X}$-ray structural data were not obtained in that period. These compounds are covered in previous reviews $[7,8]$. There is a large amount of X-ray structural data and as can be seen from Table 7 , for a fixed complex type and coordination number, the Ga-P/As bond lengths increase with halide $\mathrm{Cl}<\mathrm{Br}<\mathrm{I}$. The three complexes $\left[\mathrm{GaX}_{3}\left\{\mathrm{P}\left(\mathrm{SiMe}_{3}\right)_{3}\right\}\right]$ are anomalous in that the $\mathrm{Ga}-\mathrm{P}$ bond lengths have the reverse trend with $X$ [178], but we note that two of the structures are solvates, and it is likely that this series is a further example of the subtle effects of other contributions such as molecule-solvate interactions, which can mask the underlying trends. Despite the moderate quadrupole moment, ${ }^{71} \mathrm{Ga}$ NMR data are usually readily obtained for $\mathrm{C}_{3 \mathrm{v}}\left[\mathrm{GaX}_{3}\left(\mathrm{ER}_{3}\right)\right]$ complexes, and the gallium chemical shifts exhibit systematic changes with $\mathrm{X}$ and $\mathrm{E}$ (Table 8 ), and in some cases ${ }^{71} \mathrm{Ga}-{ }^{31} \mathrm{P}$ and ${ }^{69} \mathrm{Ga}-{ }^{31} \mathrm{P}$ couplings are resolved. However, in other symmetries including distorted octahedral, the gallium resonances have not been observed, due to fast relaxation and the resulting extreme line broadening. The phosphine complexes usually exhibit low frequency ${ }^{31} \mathrm{P}$ coordination shifts, that are also larger (i.e. more negative) with halide co-ligands $\mathrm{Cl}<\mathrm{Br}<\mathrm{I}$. As is clear from Table 8 , the dominant contribution to the ${ }^{71} \mathrm{Ga}$ chemical shift is the halide present; changing from $\mathrm{PR}_{3}$ to $\mathrm{AsR}_{3}$ or changing the $\mathrm{R}$ group has minimal effect. Recently, solid state ${ }^{69 / 71} \mathrm{Ga}$ and ${ }^{31} \mathrm{P}\left\{{ }^{1} \mathrm{H}\right\}$ NMR data have been reported for several $\left[\mathrm{GaX}_{3}\left(\mathrm{PR}_{3}\right)\right]$ complexes [177], and this reference contains a thorough discussion of the various contributions to the observed chemical shifts. Solution NMR studies in chlorocarbon solvents also reveal that some $\left[\mathrm{GaX}_{3}\left(\mathrm{ER}_{3}\right)\right]$ undergo fast ligand exchange in solution in non-coordinating solvents at room temperature or above (depending upon the specific combination of $\mathrm{ER}_{3} / \mathrm{X}$ present), and cooling the solutions may be necessary to observe resonances and spin-spin couplings $[170,171,176]$. In solution in chlorocarbon solvents, ${ }^{31} \mathrm{P}\left\{{ }^{1} \mathrm{H}\right\}$ NMR spectroscopy also identified that hydrolysis formed $\left[\mathrm{PR}_{3} \mathrm{H}\right]\left[\mathrm{GaX}_{4}\right]$ complexes, and $\left[\left\{0-\mathrm{C}_{6} \mathrm{H}_{4}\left(\mathrm{PPh}_{2}\right)\left(\mathrm{PPh}_{2} \mathrm{H}\right\}\right]\left[\mathrm{GaBr}_{4}\right]\right.$ formed in this way has been identified crystallographically [205]. In contrast to some group 14 and 15 metals, gallium does not appear to promote oxidation of phosphines to the corresponding phosphine oxides [171].

Gallium(I) and gallium(II) complexes are very rare, but recent results have demonstrated possible entry routes into this area. The first example, $\left[\mathrm{Ga}_{8} \mathrm{I}_{8}\left(\mathrm{PEt}_{3}\right)_{6}\right]$ was obtained by condensing "GaI", $\mathrm{PEt}_{3}$ and toluene vapour at $77 \mathrm{~K}$ and cautiously allowing the

Table 8

Illustrative ${ }^{71} \mathrm{Ga},{ }^{115} \mathrm{In}$ and ${ }^{31} \mathrm{P}\left\{{ }^{1} \mathrm{H}\right\}$ NMR data for gallium and indium complexes $[170,171,179,227,228,232]$.

\begin{tabular}{|c|c|c|c|c|}
\hline Complex & $\delta\left({ }^{71} \mathrm{Ga} /{ }^{115} \mathrm{In}\right)^{\mathrm{a}}$ & $\delta\left({ }^{31} \mathrm{P}\right)^{\mathrm{b}}$ & $\Delta \mathrm{P}^{\mathrm{c}}$ & ${ }^{1} J\left({ }^{71} \mathrm{Ga}-{ }^{31} \mathrm{P}\right)(\mathrm{Hz})$ \\
\hline$\left[\mathrm{GaCl}_{3}\left(\mathrm{PPh}_{3}\right)\right]$ & 264 & -5.4 & +0.6 & 721 \\
\hline$\left[\mathrm{GaBr}_{3}\left(\mathrm{PPh}_{3}\right)\right]$ & 152 & -10.7 & -4.7 & 693 \\
\hline$\left[\mathrm{GaI}_{3}\left(\mathrm{PPh}_{3}\right)\right]$ & -151 & -29.7 & -23.7 & 466 \\
\hline$\left[\mathrm{GaCl}_{3}\left(\mathrm{AsPh}_{3}\right)\right]$ & 264 & & & \\
\hline$\left[\mathrm{GaBr}_{3}\left(\mathrm{AsPh}_{3}\right)\right]$ & 132 & & & \\
\hline$\left[\mathrm{GaI}_{3}\left(\mathrm{AsPh}_{3}\right)\right]$ & -203 & & & \\
\hline$\left[\mathrm{GaCl}_{3}\left(\mathrm{AsMe}_{3}\right)\right]$ & 264.5 & & & \\
\hline$\left[\mathrm{GaBr}_{3}\left(\mathrm{AsMe}_{3}\right)\right]$ & 147 & & & \\
\hline$\left[\mathrm{GaI}_{3}\left(\mathrm{AsMe}_{3}\right)\right]$ & -169.5 & & & \\
\hline$\left[\mathrm{InI}_{3}\left(\mathrm{PPh}_{3}\right)\right]$ & Not observed & -22 & -16 & \\
\hline$\left[\mathrm{InI}_{3}\left(\mathrm{PH}^{\mathrm{t}} \mathrm{Bu}_{2}\right)\right]$ & -228 & 3 & -20 & \\
\hline$\left[\mathrm{InI}_{3}\left\{o-\mathrm{C}_{6} \mathrm{H}_{4}\left(\mathrm{PPh}_{2}\right)_{2}\right\}\right]$ & Not observed & -31.7 & -19 & \\
\hline$\left[\operatorname{InI}_{2}\left\{o-\mathrm{C}_{6} \mathrm{H}_{4}\left(\mathrm{PPh}_{2}\right)_{2}\right\}\right]\left[\mathrm{InI}_{4}\right]$ & Not observed & -28 & -15 & \\
\hline$\left[\mathrm{InI}_{2}\left\{0-\mathrm{C}_{6} \mathrm{H}_{4}\left(\mathrm{PMe}_{2}\right)_{2}\right\}\right]\left[\mathrm{InI}_{4}\right]$ & Not observed & -42 & +13 & \\
\hline$\left[\mathrm{InCl}_{2}\left\{o-\mathrm{C}_{6} \mathrm{H}_{4}\left(\mathrm{PMe}_{2}\right)_{2}\right\}\right]\left[\mathrm{InCl}_{4}\right]$ & Not observed & -36.5 & +18.5 & \\
\hline
\end{tabular}

a Relative to $\left[\mathrm{Ga}\left(\mathrm{H}_{2} \mathrm{O}\right)_{6}\right]^{3+}$ or $\left[\operatorname{In}\left(\mathrm{H}_{2} \mathrm{O}\right)_{6}\right]^{3+}$ in water.

b Relative to $85 \% \mathrm{H}_{3} \mathrm{PO}_{4}$.

c Coordination shift. 


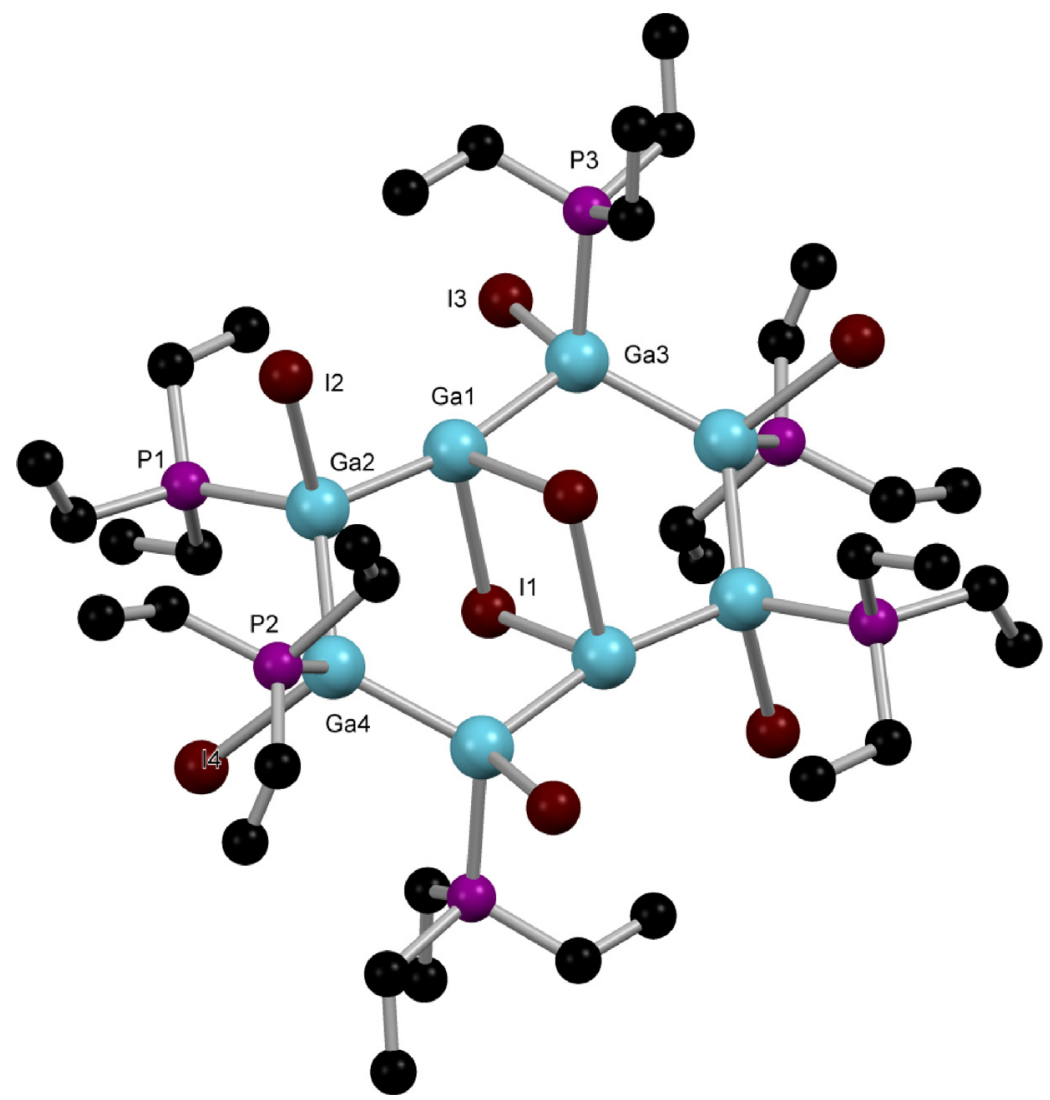

Fig. 24. View of the structure of $\left[\mathrm{Ga}_{8} \mathrm{I}_{8}\left(\mathrm{PEt}_{3}\right)_{6}\right]$ redrawn from Ref. [183].

mixture to thaw [183]. The structure reveals a planar $\mathrm{Ga}_{8}$ ring with two galliums bridged by iodides, and the remaining six gallium centres coordinated to terminal iodide and $\mathrm{PEt}_{3}$ groups (Fig. 24). The formation of a Ga 8 ring contrasts with the $\mathrm{Al}_{4}$ ring discussed above; although the reasons for the difference are not completely clear, both steric and electronic factors seem to be involved. More conventional syntheses from $\left[\mathrm{Ga}(\mathrm{PhMe})_{2}\right]\left[\mathrm{Al}\left\{\mathrm{OC}\left(\mathrm{CF}_{3}\right)_{3}\right\}_{4}\right]$ and $\mathrm{PPh}_{3}$ or $\mathrm{P}^{t} \mathrm{Bu}_{3}$ in $\mathrm{C}_{6} \mathrm{H}_{4} \mathrm{~F}_{2}$ gave $\left[\mathrm{Ga}\left(\mathrm{PPh}_{3}\right)_{3}\right]\left[\mathrm{Al}\left\{\mathrm{OC}\left(\mathrm{CF}_{3}\right)_{3}\right\}_{4}\right]$ and $\left[\mathrm{Ga}\left(\mathrm{P}^{t} \mathrm{Bu}_{3}\right)_{2}\right]\left[\mathrm{Al}\left\{\mathrm{OC}\left(\mathrm{CF}_{3}\right)_{3}\right\}_{4}\right]$, respectively $[184,185]$.

Several examples of formally $\mathrm{Ga}$ (II) compounds with unbridged $\mathrm{Ga}-\mathrm{Ga}$ bonds have been prepared. Ultrasonication of $\mathrm{Ga}$ and $\mathrm{I}_{2}$ in toluene, followed by addition of $\mathrm{PEt}_{3}$ at $-78{ }^{\circ} \mathrm{C}$ gave two compounds, $\left[\mathrm{Ga}_{2} \mathrm{I}_{4}\left(\mathrm{PEt}_{3}\right)_{2}\right]$ and $\left[\mathrm{Ga}_{3} \mathrm{I}_{5}\left(\mathrm{PEt}_{3}\right)_{3}\right]$, the latter shown by an X-ray study to be $\left[\mathrm{I}_{2}\left(\mathrm{PEt}_{3}\right) \mathrm{Ga}-\mathrm{GaI}\left(\mathrm{PEt}_{3}\right)-\mathrm{GaI}_{2}\left(\mathrm{PEt}_{3}\right)_{2}\right]$ (Fig. 25) [186]. Treatment of $\mathrm{Ga}\left[\mathrm{GaX}_{4}\right](\mathrm{X}=\mathrm{Cl}$ or $\mathrm{Br})$ with $\mathrm{PEt}_{3}$ produces $\left[\mathrm{Ga}_{2} \mathrm{X}_{4}\left(\mathrm{PEt}_{3}\right)_{2}\right]$ [172], whilst $\left[\mathrm{Ga}_{2} \mathrm{I}_{4}\left(\mathrm{AsEt}_{3}\right)_{2}\right]$ is formed from gallium metal and $\mathrm{Et}_{3} \mathrm{AsI}_{2}$ (Fig. 26) [187]. Curiously, gallium powder and $\left(p-\mathrm{MeOC}_{6} \mathrm{H}_{4}\right)_{3} \mathrm{AsI}_{2}$ produced the "normal" $\mathrm{Ga}$ (III) complex $\left[\mathrm{GaI}_{3}\left\{\mathrm{As}\left(p-\mathrm{MeOC}_{6} \mathrm{H}_{4}\right)_{3}\right\}\right.$ ] [187]. The Ga-Ga bonds are very similar along the series of dimers $(\sim 2.43 \AA)$, although slightly longer (2.45(1), 2.46(1) $\AA$ ) in the Ga $\mathrm{Ga}_{3}$ complex. Reaction of "Gal", prepared by ultrasonication, with secondary phosphines also gave $\left[\mathrm{Ga}_{2} \mathrm{I}_{4}\left(\mathrm{PHR}_{2}\right)_{2}\right]\left(\mathrm{R}={ }^{t} \mathrm{Bu}\right.$ or $\left.\mathrm{Cy}\right)[188]$.

For typical $\mathrm{Ga}$ (III) complexes, consideration of the structural data in Table 7 shows that for $\left[\mathrm{GaX}_{3}\left(\mathrm{ER}_{3}\right)\right](\mathrm{E}=\mathrm{P}$ or As) the Ga-E distances increase along the series $\mathrm{Cl}<\mathrm{Br}<\mathrm{I}$ as noted previously. However, there seem to be only small effects on the Ga-E bond lengths from changing the $\mathrm{R}$ group, although in analogous complexes with $\mathrm{R}=$ aryl the Ga-E seem to be marginally longer than $\mathrm{R}=$ alkyl [170-177], which is in keeping both with the Ga-X bonds being dominant compared to Ga-E, and also with the absence of significant steric effects in these complexes. There appear to be no established examples of gallium(III) halide complexes of stibines; early studies suggested fragmentation of the stibine or X/R exchange [7]. The nearest approach seems to be $\left[\mathrm{GaPhI}_{2}\left(\mathrm{SbPh}_{3}\right)\right]$ [204] made from "GaI" and $\mathrm{SbPh}_{3}$, in a reaction clearly involving $\mathrm{Sb}-\mathrm{C}$ bond cleavage.

Flexible bidentate diphosphino- and diarsino-alkanes also generate distorted tetrahedral complexes with the ligands bridging two gallium centres as in $\left[\mathrm{X}_{3} \mathrm{Ga}(\mu-\mathrm{L}-\mathrm{L}) \mathrm{GaX}_{3}\right]$ $\left(\mathrm{L}-\mathrm{L}=\mathrm{Ph}_{2} \mathrm{P}\left(\mathrm{CH}_{2}\right)_{2} \mathrm{PPh}_{2}, \quad \mathrm{Ph}_{2} \mathrm{As}\left(\mathrm{CH}_{2}\right)_{2} \mathrm{AsPh}_{2}, \quad \mathrm{Et}_{2} \mathrm{P}\left(\mathrm{CH}_{2}\right)_{2} \mathrm{PEt}_{2}\right)$ (Fig. 27); it is notable that even with $\mathrm{Et}_{2} \mathrm{P}\left(\mathrm{CH}_{2}\right)_{2} \mathrm{PEt}_{2}$, a strong $\sigma$-donor which usually bonds as a chelate to $3 \mathrm{~d}$ transition metals,

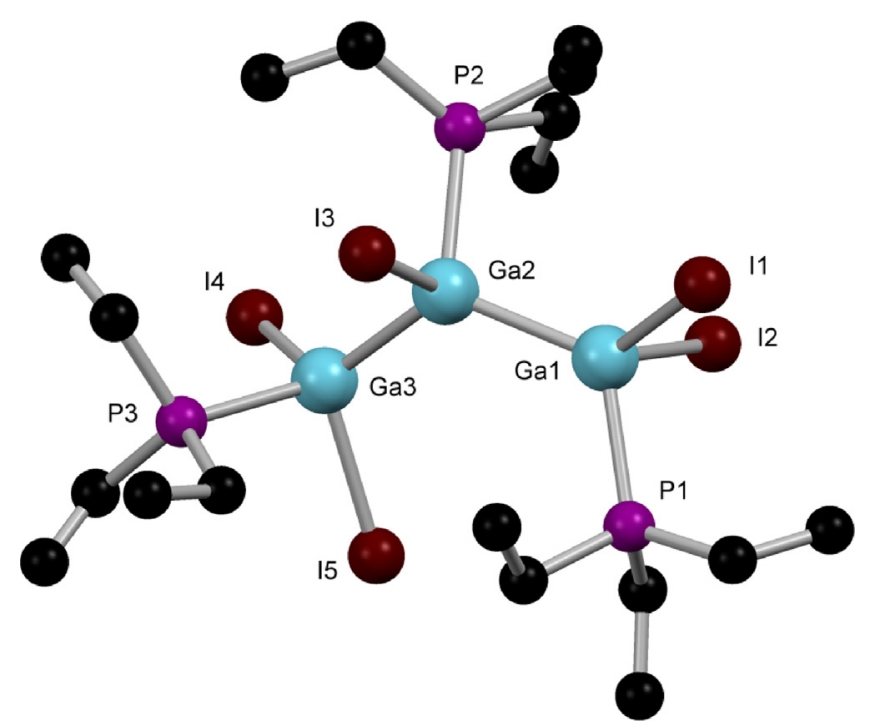

Fig. 25. View of the structure of $\left[\mathrm{I}_{2}\left(\mathrm{PEt}_{3}\right) \mathrm{Ga}-\mathrm{GaI}\left(\mathrm{PEt}_{3}\right)-\mathrm{GaI}_{2}\left(\mathrm{PEt}_{3}\right)_{2}\right]$ redrawn from Ref. [186]. 


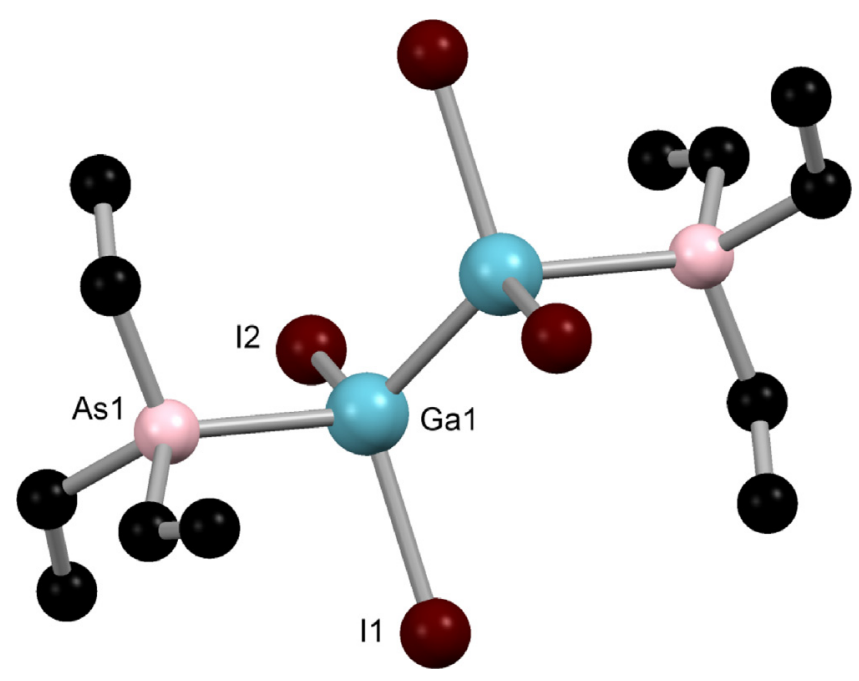

Fig. 26. View of the structure of $\left[\mathrm{Ga}_{2} \mathrm{I}_{4}\left(\mathrm{AsEt}_{3}\right)_{2}\right]$ redrawn from Ref. [187].

there is no evidence for the formation of $\left[\mathrm{GaX}_{2}\left\{\mathrm{Et}_{2} \mathrm{P}\left(\mathrm{CH}_{2}\right)_{2} \mathrm{PEt}_{2}\right\}\right]^{+}$ $[170,176,180]$. The diphosphinoalkene cis- $\mathrm{Ph}_{2} \mathrm{PCH}=\mathrm{CHPPh}_{2}$ reacts with $\mathrm{GaX}_{3}(\mathrm{X}=\mathrm{Br}$ or $\mathrm{I})$ in hot toluene to form $\left[\mathrm{X}_{3} \mathrm{Ga}(\mu\right.$-trans$\left.\left.\mathrm{Ph}_{2} \mathrm{PCH}=\mathrm{CHPPh}_{2}\right) \mathrm{GaX}_{3}\right]$, the cis $\rightarrow$ trans isomerisation being confirmed spectroscopically and by the X-ray structure of the bromide complex [181].

The semi-rigid, but sterically bulky, o-xylyl diphosphine, $o-\mathrm{C}_{6} \mathrm{H}_{4}\left(\mathrm{CH}_{2} \mathrm{PPh}_{2}\right)_{2}$ also forms only bridged dimers $\left[\mathrm{X}_{3} \mathrm{Ga}\{0\right.$ $\left.\mathrm{C}_{6} \mathrm{H}_{4}\left(\mathrm{CH}_{2} \mathrm{PPh}_{2}\right)_{2}\right\} \mathrm{GaX}_{3}$ ], the structure for $\mathrm{X}=\mathrm{I}$ showing the $\mathrm{GaI}_{3}$ groups disposed "anti", presumably to minimise steric interactions [171]. However, the rigid o-phenylene diphosphines $o-\mathrm{C}_{6} \mathrm{H}_{4}\left(\mathrm{PR}_{2}\right)_{2}$ chelate to gallium, displacing one $\mathrm{X}^{-}$in the process. The bulky $o-\mathrm{C}_{6} \mathrm{H}_{4}\left(\mathrm{PPh}_{2}\right)_{2}$ forms distorted tetrahedral cations in $\left[\mathrm{GaX}_{2}\{0-\right.$ $\left.\left.\mathrm{C}_{6} \mathrm{H}_{4}\left(\mathrm{PPh}_{2}\right)_{2}\right\}\right]\left[\mathrm{GaX}_{4}\right]$ (Fig. 28) which are the only forms isolated in the solid state, although in situ ${ }^{31} \mathrm{P}\left\{{ }^{1} \mathrm{H}\right\}$ NMR studies suggested other species may be present in solution in the presence of excess diphosphine. The structures of the cations are distorted by the small chelate bite of the diphosphine $\left(<\mathrm{P}-\mathrm{Ga}-\mathrm{P} \sim 85^{\circ}\right)$, and the distortions cause loss of the ${ }^{71} \mathrm{Ga}$ NMR resonances of the cations as a consequence of the increased electric field gradients and resulting fast relaxation $[170,179]$. The rigid, but smaller and stronger $\sigma$-donor diphosphine, $o-\mathrm{C}_{6} \mathrm{H}_{4}\left(\mathrm{PMe}_{2}\right)_{2}$, produced the only known examples of six-coordination in gallium phosphines, in trans-[GaX $\left.\mathrm{Ga}_{2}\left\{\mathrm{C}_{6} \mathrm{H}_{4}\left(\mathrm{PMe}_{2}\right)_{2}\right\}_{2}\right]\left[\mathrm{GaX}_{4}\right]$ (Fig. 29) [170]. The Ga-P and $\mathrm{Ga}-\mathrm{X}$ distances are longer than in the tetrahedral $\mathrm{GaX}_{3} \mathrm{P}$ species due to the increased coordination number, but the Ga-P distances differ only slightly with changes in X. The distortions from cubic symmetry are again sufficient to cause loss of the ${ }^{71} \mathrm{Ga}$ NMR resonances of the cations, and the coordination shifts in the ${ }^{31} \mathrm{P}\left\{{ }^{1} \mathrm{H}\right\}$ NMR spectra are erratic. The reaction of $\mathrm{GaCl}_{3}$ with $0-\mathrm{C}_{6} \mathrm{H}_{4}\left(\mathrm{PMe}_{2}\right)_{2}$ in a 2:1 molar ratio produced an insoluble complex which was probably $\left[\mathrm{GaX}_{2}\left\{0-\mathrm{C}_{6} \mathrm{H}_{4}\left(\mathrm{PMe}_{2}\right)_{2}\right\}\right]\left[\mathrm{GaX}_{4}\right]$ containing a distorted tetrahedral cation, although $\mathrm{X}$-ray structural authentication is lacking [170]. The original report of complexes of the diarsine, $o-\mathrm{C}_{6} \mathrm{H}_{4}\left(\mathrm{AsMe}_{2}\right)_{2}$, also suggested these contained distorted octahedral cations based upon microanalysis, MWt and conductance measurements [206]. However, re-investigation isolated $\left[\mathrm{GaX}_{2}\left\{o-\mathrm{C}_{6} \mathrm{H}_{4}\left(\mathrm{AsMe}_{2}\right)_{2}\right\}\right]\left[\mathrm{GaX}_{4}\right](\mathrm{X}=\mathrm{Cl}, \mathrm{Br}$ or I) [170], all shown to be distorted tetrahedral cations by spectroscopy and their $\mathrm{X}$-ray structures. If an excess of the ligand is used in the syntheses, other complexes are formed, probably $\left[\mathrm{GaX}_{2}\left\{0-\mathrm{C}_{6} \mathrm{H}_{4}\left(\mathrm{AsMe}_{2}\right)_{2}\right\}\right] \mathrm{X}$, although these were not obtained in a pure state; but no evidence for six-coordinate cations was found [170].

The bulky and rather rigid triphosphine $\mathrm{PhP}\left(o-\mathrm{C}_{6} \mathrm{H}_{4} \mathrm{PPh}_{2}\right)_{2}$ gave $\left[\mathrm{GaI}_{2}\left\{\mathrm{PhP}\left(0-\mathrm{C}_{6} \mathrm{H}_{4} \mathrm{PPh}_{2}\right)_{2}\right\}\right]\left[\mathrm{GaI}_{4}\right]$ on reaction with $\mathrm{GaI}_{3}$ in toluene, its X-ray structure (Fig. 30 ) showing a distorted tetrahedral cation with the terminal $-\mathrm{PPh}_{2}$ groups coordinated to the gallium. The central PhP is $\sim 2.9 \AA$ from gallium and incorrectly orientated for a third Ga-P bond to be present [179]. Unexpectedly the tripodal triarsine, $\mathrm{MeC}\left(\mathrm{CH}_{2} \mathrm{AsMe}_{2}\right)_{3}$, produced three different types of complexes with gallium(III) halides, although all contain distorted tetrahedral gallium centres [182]. In [ $\left.\left(\mathrm{GaI}_{3}\right)_{3}\left\{\mathrm{MeC}\left(\mathrm{CH}_{2} \mathrm{AsMe}_{2}\right)_{3}\right\}\right]$ (Fig. 31(a)) each arsenic is coordinated to a different gallium, whilst in $\left[\mathrm{GaCl}_{2}\left\{\mathrm{MeC}\left(\mathrm{CH}_{2} \mathrm{AsMe}_{2}\right)_{3}\right\}\right]\left[\mathrm{GaCl}_{4}\right]$ (Fig. 31(b)), a GaAs${ }_{2} \mathrm{Cl}_{2}$ cation is present, with the third arsine donor atom uncoordinated. In $\left[\left(\mathrm{GaCl}_{3}\right)\left(\mathrm{GaCl}_{2}\right)\left\{\mathrm{MeC}\left(\mathrm{CH}_{2} \mathrm{AsMe}_{2}\right)_{3}\right\}\right]\left[\mathrm{GaCl}_{4}\right]$ (Fig. 31(c)) the third arsenic donor is coordinated to a neutral $\mathrm{GaCl}_{3}$ group [182].

Gallane adducts $\left[\mathrm{GaH}_{3}\left(\mathrm{PR}_{3}\right)\right] \quad\left(\mathrm{R}=\mathrm{Me}, \mathrm{Cy}\right.$ or $\left.{ }^{t} \mathrm{Bu}\right)$ and $\left[\left(\mathrm{GaH}_{3}\right)_{2}(\mu-\mathrm{L}-\mathrm{L})\right] \quad\left(\mathrm{L}-\mathrm{L}=\mathrm{Me}_{2} \mathrm{P}\left(\mathrm{CH}_{2}\right)_{2} \mathrm{PMe}_{2},{ }^{i} \mathrm{Pr}_{2} \mathrm{P}\left(\mathrm{CH}_{2}\right)_{2} \mathrm{P}^{\mathrm{i}} \mathrm{Pr}_{2}\right.$ or $\left.\mathrm{Ph}_{2} \mathrm{P}\left(\mathrm{CH}_{2}\right)_{2} \mathrm{PPh}_{2}\right)$ are formed from $\mathrm{LiGaH}_{4}, \mathrm{HCl}$ and the phosphine in diethyl ether or by displacement of the amine from $\left[\mathrm{GaH}_{3}\left(\mathrm{NMe}_{3}\right)\right][137,207-209]$. The complexes are more thermally stable than the alane analogues, and $\left[\mathrm{GaH}_{3}\left(\mathrm{PR}_{3}\right)\right]$ can be sublimed

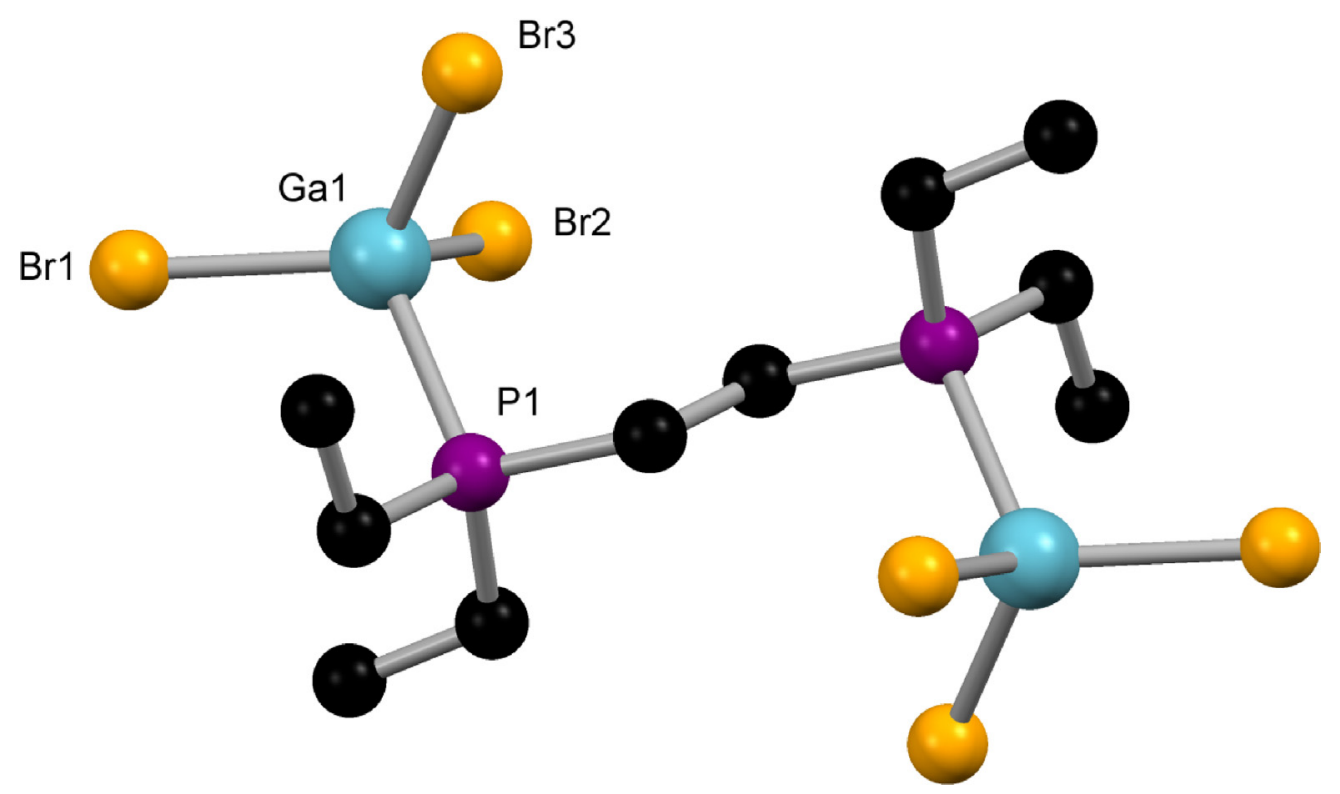

Fig. 27. View of the structure of $\left[\left(\mathrm{GaBr}_{3}\right)_{2}\left\{\mu-\mathrm{Et}_{2} \mathrm{P}\left(\mathrm{CH}_{2}\right)_{2} \mathrm{PEt}_{2}\right\}\right]$ redrawn from Ref. [170]. 


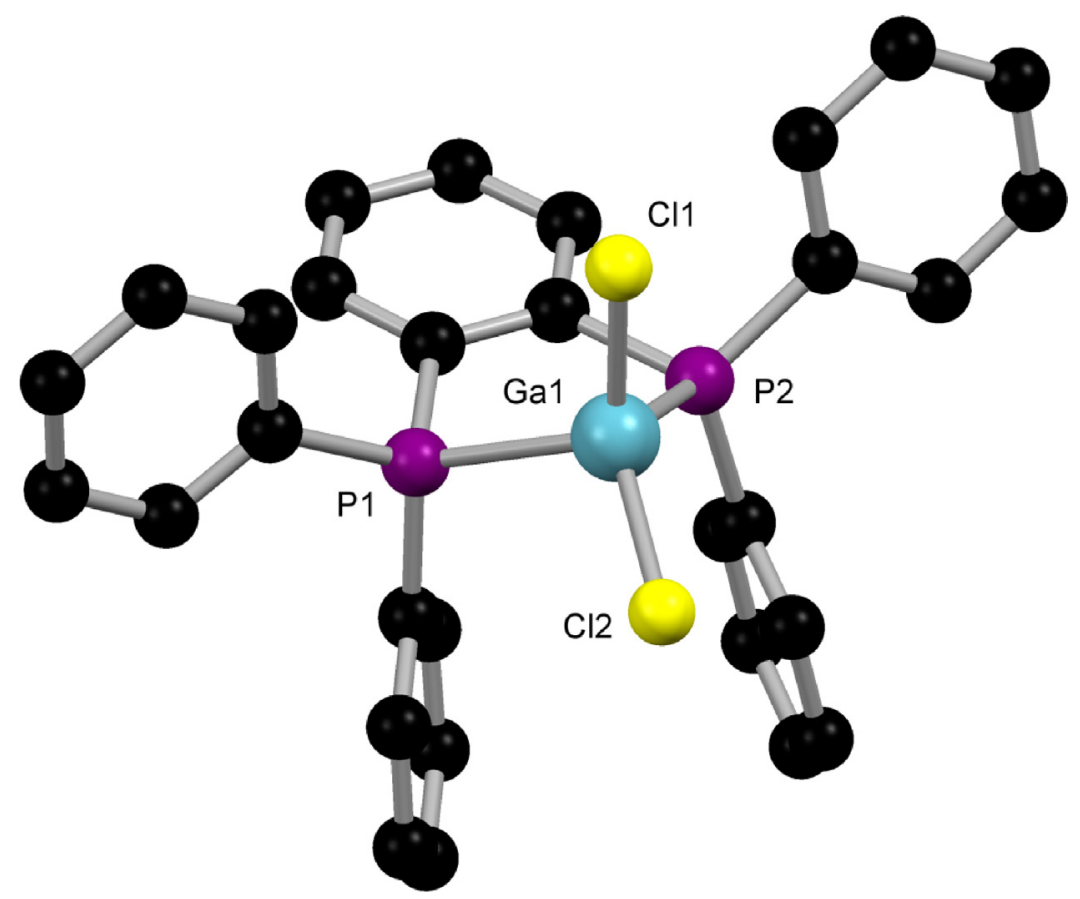

Fig. 28. View of the structure of the cation $\left[\mathrm{GaCl}_{2}\left\{0-\mathrm{C}_{6} \mathrm{H}_{4}\left(\mathrm{PPh}_{2}\right)_{2}\right\}\right]^{+}$redrawn from Ref. [170].

in vacuo. Comparison of Ga-P bond lengths between $\left[\mathrm{GaH}_{3}\left(\mathrm{PR}_{3}\right)\right]$ and $\left[\mathrm{GaCl}_{3}\left(\mathrm{PR}_{3}\right)\right]$ show the latter to be somewhat shorter, consistent with higher Lewis acidity of the gallium chloride. The complex $\left[\mathrm{GaH}_{3}\left(\mathrm{PMe}_{3}\right)\right]$ has been studied as single crystals by $\mathrm{X}$-ray diffraction, by electron diffraction in the gas phase, and by various quantum mechanical calculations [207]. Given the two experimental techniques are measuring slightly different parameters and there is a significant temperature difference in the data collections, agreement is very good, with Ga-P differing only by $\sim 0.06 \AA$, but this does again emphasise the care that should be taken in interpreting very small differences in bond lengths (in different complexes) as significant differences in Lewis acidity.

Complexes of halogallanes are also known [172,210-212]. Synthesis routes involve redistribution between $\left[\mathrm{GaCl}_{3}\left(\mathrm{PCy}_{3}\right)\right]$ and $\left[\mathrm{GaH}_{3}\left(\mathrm{PCy}_{3}\right)\right]$, which gives $\left[\mathrm{GaHCl}_{2}\left(\mathrm{PCy}_{3}\right)\right]$ and $\left[\mathrm{GaH}_{2} \mathrm{Cl}\left(\mathrm{PCy}_{3}\right)\right]$, and reaction of $\left[\mathrm{GaH}_{3}\left(\mathrm{PR}_{3}\right)\right]$ or $\mathrm{Li}\left[\mathrm{GaH}_{4}\right]$ and $\mathrm{PR}_{3}$ with $\mathrm{HCl}$. Direct substitution of the phosphine into $\left[\mathrm{Ga}_{2} \mathrm{H}_{2} \mathrm{Cl}_{4}\right]$ is also possible [172,212].

Trialkylgallium adducts are known for all $\mathrm{ER}_{3}(\mathrm{E}=\mathrm{P}, \mathrm{As}, \mathrm{Sb}$ or $\mathrm{Bi}$ ) donor types and as expected generally resemble the aluminium analogues. The syntheses are straightforward involving combining the $\mathrm{GaR}_{3}$ with $\mathrm{ER}_{3}$ either in a hydrocarbon or without a solvent $[141,194,196,198,199,201]$. Triarylgallium examples are fewer but generally similar [198,203]. Di- and poly-phosphine adducts are also known $[141,146,202]$ and all contain only a single phosphorus donor bonded to each gallium centre. The adducts with involatile phosphines have been used to store the $\mathrm{GaR}_{3}$, and since the $\mathrm{GaR}_{3}$ is liberated on heating in vacuo, this also affords a means of purifying the $\mathrm{GaR}_{3}$ for electronic materials applications [141,146]. A considerable number of compounds have been characterised by X-ray diffraction (Table 7), all contain four-coordinate distorted tetrahedral gallium. The Ga-P/As bond lengths are generally longer than in the gallium(III) halide adducts, and large R-groups on the Ga or the E-donor tend to lengthen the Ga-E bond. A range of examples with $\mathrm{SbR}_{3}$ and $\mathrm{BiR}_{3}$ is also known (Fig. 32), although these are particularly sensitive compounds, and contain much weaker Ga-E bonds than the lighter Group 15 analogues [149,154,192-195,200]. Phosphine and arsine adducts of $\mathrm{R}_{2} \mathrm{GaCl}\left(\mathrm{R}=\mathrm{Me}, \mathrm{Me}_{3} \mathrm{CCH}_{2}, \mathrm{Ph}\right)$ have been prepared $[197,198,202]$. There are also examples of

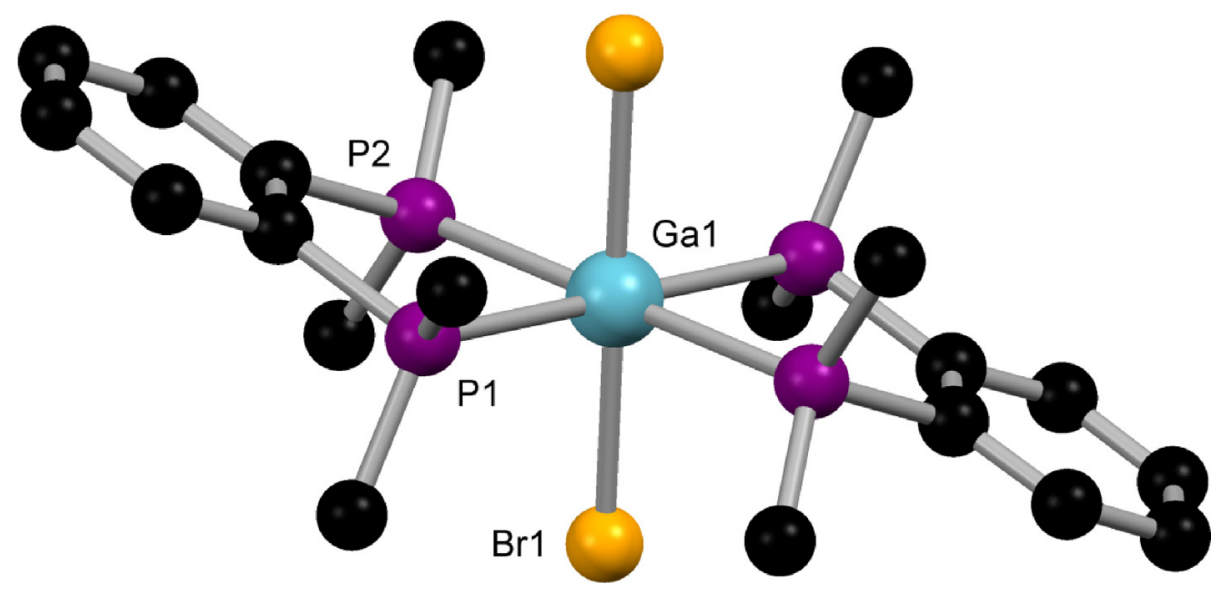

Fig. 29. View of the structure of the cation $\left[\mathrm{GaBr}_{2}\left\{0-\mathrm{C}_{6} \mathrm{H}_{4}\left(\mathrm{PMe}_{2}\right)_{2}\right\}_{2}\right]^{+}$redrawn from ref. [170]. 
alkyl or aryl ligands containing phosphine pendant groups. In the (diphenylphosphinomethyl)phenyl complex $\left[\mathrm{Ga}\left(\mathrm{Ph}_{2} \mathrm{PCH}_{2} \mathrm{C}_{6} \mathrm{H}_{4}\right)_{3}\right]$, the geometry is distorted tetrahedral $\left(\mathrm{C}_{3} \mathrm{P}\right)$, in contrast to the aluminium and indium complexes which are five-coordinate [162]. Chelating 3-(di-t-butylphosphino)propyl groups are present in $\left[\mathrm{Me}_{2} \mathrm{Ga}\left\{\left(\mathrm{CH}_{2}\right)_{3} \mathrm{P}^{t} \mathrm{Bu}_{2}\right\}\right]$ and $\left[\mathrm{Cl}_{2} \mathrm{Ga}\left\{\left(\mathrm{CH}_{2}\right)_{3} \mathrm{P}^{t} \mathrm{Bu}_{2}\right\}\right]$ [213], but $-\mathrm{CH}_{2} \mathrm{PPh}_{2}$ groups in $\left[\left\{\mathrm{R}_{2} \mathrm{Ga}\left(\mathrm{CH}_{2} \mathrm{PPh}_{2}\right)\right\}_{2}\right]\left(\mathrm{R}=\mathrm{CH}_{2} \mathrm{CMe}_{3}, \mathrm{CH}_{2} \mathrm{SiMe}_{3}\right)$ bridge the galliums giving dimeric species with $\mathrm{C}_{3} \mathrm{P}$ coordination [214]. Chelation is present in [GaMe $\left.2\left\{\mathrm{C}_{5} \mathrm{H}_{4}\left(\mathrm{CH}_{2}\right)_{2} \mathrm{P}^{t} \mathrm{Bu}_{2}\right\}\right]$ and $\left[\mathrm{GaCl}_{2}\left\{\mathrm{C}_{5} \mathrm{H}_{4}\left(\mathrm{CH}_{2}\right)_{2} \mathrm{P}^{t} \mathrm{Bu}_{2}\right\}\right]$, which have $\eta^{1}$-coordination in the cyclopentadienyl ring, and hence distorted tetrahedral gallium [215]. Chelating C,P-coordination is found in closo2-[(dimethylphosphino)methyl]-1,2-dicarbaborane (HCB) complexes $\left[\mathrm{GaCl}_{2}(\mathbf{C B})\right]$ and $\left[\mathrm{GaCl}(\mathbf{C B})_{2}\right]$ and the corresponding methylgallium complexes [165].

Gallium(III) complexes with various anionic chelating ligands incorporating alkoxide, thiolate or amido donor groups in addition to neutral Group 15 donor atoms have also received attention recently. These include $\mathrm{Ph}_{2} \mathrm{ECH}_{2} \mathrm{CHRO}^{-}$and $\mathrm{Ph}_{2} \mathrm{ECH}_{2} \mathrm{CHRS}^{-}$ $(\mathrm{E}=\mathrm{P}, \mathrm{Sb}$ or $\mathrm{Bi})$, which form adducts with $\mathrm{Ga}^{i} \mathrm{Pr}_{2}$ groups [216]; their characterisation relies mainly on NMR spectroscopy and so structural data for the heavier examples would be valuable. The amino-diphosphine (PNP) pincer (III-H) bonds as a tridentate monoanion with deprotonated $\mathrm{N}\left(\mathrm{NP}_{2}\right.$ coordination) in trigonal bipyramidal $\left[\mathrm{GaCl}_{2}(\mathrm{III})\right]$, but as a bidentate $\left(\mathrm{P}_{2}\right)$ donor in its neutral form in $\left[\mathrm{GaX}_{2}\right.$ (III-H)] $\left[\mathrm{GaX}_{4}\right](\mathrm{X}=\mathrm{Cl}$ or $\mathrm{Br})$ (Fig. 33) [217]. It also reacts with "Gal" to form $\left[\mathrm{Ga}_{2} \mathrm{I}_{2}(\mathrm{III})_{2}\right]$ in which a $\mathrm{Ga}-\mathrm{Ga}$ bond is present with each gallium coordinated to a terminal iodide and (III) as a NP chelate [217]. The macrocyclic diamidodiphosphine $\left(\mathbf{P}_{\mathbf{2}} \mathbf{N}_{\mathbf{2}}\right)$ (see Section 6.2) forms syn and anti isomers of $\left[\mathrm{GaCl}\left(\mathbf{P}_{\mathbf{2}} \mathbf{N}_{\mathbf{2}}\right)\right]$ and syn- $\left[\mathrm{GaH}\left(\mathbf{P}_{\mathbf{2}} \mathbf{N}_{\mathbf{2}}\right)\right]$, analogous to the aluminium complexes discussed above. However, for gallium, reduction of syn- $\left[\mathrm{GaCl}\left(\mathbf{P}_{\mathbf{2}} \mathbf{N}_{\mathbf{2}}\right)\right]$ with $\mathrm{KC}_{8}$ produces the dimer $\left[\left\{\mathrm{Ga}\left(\mathbf{P}_{\mathbf{2}} \mathbf{N}_{\mathbf{2}}\right)\right\}_{2}\right]$, which has an unsupported Ga-Ga bond $[168,169]$. A variety of coordination modes are present in the gallium(III) complexes of $o-\mathrm{C}_{6} \mathrm{H}_{4} \mathrm{PPh}_{2}(\mathrm{SH}), o-\mathrm{C}_{6} \mathrm{H}_{4} \mathrm{AsPh}_{2}(\mathrm{SH})$ and $\mathrm{PhP}\left(o-\mathrm{C}_{6} \mathrm{H}_{4} \mathrm{SH}\right)_{2}$, with the majority containing deprotonated thiolates [218,219].

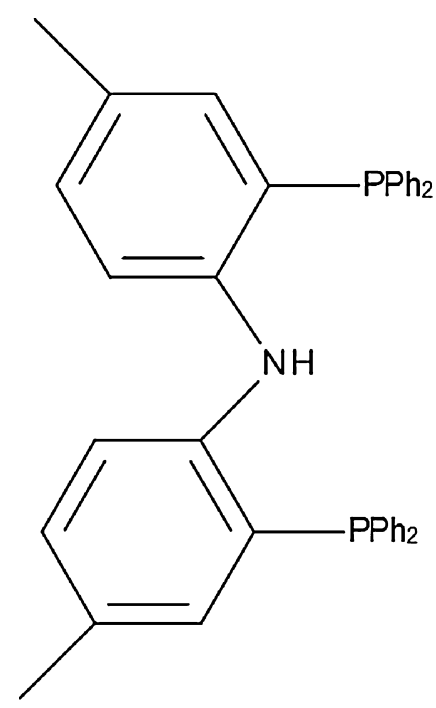

\section{(III-H)}

\subsection{Indium}

The main similarity between the pnictogen complexes of indium and its lighter analogues, aluminium and gallium, is that the vast majority of the complexes contain the M(III) oxidation state, with

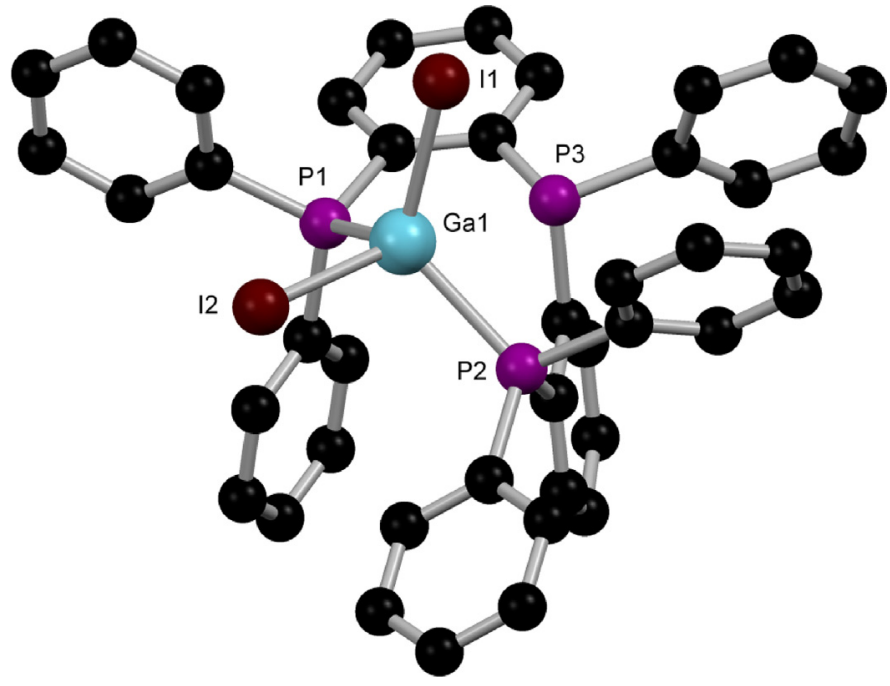

Fig. 30. View of the structure of the cation $\left[\mathrm{GaI}_{2}\left\{\mathrm{PhP}\left(o-\mathrm{C}_{6} \mathrm{H}_{4} \mathrm{PPh}_{2}\right)_{2}\right\}\right]^{+}$redrawn from Ref. [179].

very few examples of $\operatorname{In}(\mathrm{I})$ and $\mathrm{In}(\mathrm{II})$ complexes. For In(III), complexes with halide and alkyl co-ligands are common, in addition to some mixed donor bi- and multi-dentate ligands. The major differences are that $\operatorname{In}(\mathrm{III})$ has a significantly larger covalent radius of $1.50 \AA$, compared to $1.25 \AA$ for $\mathrm{Al}$ and $\mathrm{Ga}$, and that four-coordination is no longer dominant, with many examples of five- and sixcoordinate complexes established. Indium is also a weaker Lewis acid. Studies described in previous reviews $[7,8]$ showed that with monodentate phosphines, complexes of the type $\left[\operatorname{InX} X_{3}\left(\mathrm{PR}_{3}\right)_{2}\right]$ were readily formed, and there were some reports of $\left[\operatorname{InX} X_{3}\left(\mathrm{PR}_{3}\right)\right]$ and $\left[\operatorname{InX}_{3}\left(\mathrm{PR}_{3}\right)_{3}\right]$. There were also some complexes with diphosphines and diarsines whose structures were unclear, and in contrast to the lighter Group 13 metals, complexes of oxoanions such as $\left[\mathrm{In}\left(\mathrm{PR}_{3}\right)_{4}\right]\left[\mathrm{ClO}_{4}\right]_{3}$. These earlier studies are only mentioned in passing in the present article, although some key X-ray structures are included in Table 9 for completeness.

The chemistry of indium in oxidation states <III has been rapidly developing in the last twenty years [220], although very little of the work has involved heavy group 15 ligands. The reaction of $\mathrm{Ag}\left[\mathrm{Al}\left\{\mathrm{OC}\left(\mathrm{CF}_{3}\right)_{3}\right\}_{4}\right]$ with indium metal in $\mathrm{C}_{6} \mathrm{H}_{5} \mathrm{~F}$, followed by addition of $\mathrm{PPh}_{3}$, gave yellow crystals of composition $\left[\mathrm{In}\left(\mathrm{PPh}_{3}\right)_{3}\right]\left[\mathrm{Al}\left\{\mathrm{OC}\left(\mathrm{CF}_{3}\right)_{3}\right\}_{4}\right] \cdot 0.5 \mathrm{PPh}_{3} \cdot 3.5 \mathrm{C}_{6} \mathrm{H}_{5} \mathrm{~F}$ [185]. This contains two trigonal pyramidal $\left[\mathrm{In}\left(\mathrm{PPh}_{3}\right)_{3}\right]^{+}$units, with the seventh $\mathrm{PPh}_{3}$ uncoordinated in the lattice (Fig. 34). The bulkier $\mathrm{P}^{\mathrm{t}} \mathrm{Bu}_{3}$ formed the $\mathrm{V}$-shaped $\left[\operatorname{In}\left(\mathrm{P}^{t} \mathrm{Bu}_{3}\right\}_{2}\right]^{+}$with $<\mathrm{P}-\mathrm{In}-\mathrm{P}=117^{\circ}[185]$. The reactions of $\mathrm{In}_{2} \mathrm{X}_{4}\left(\mathrm{X}=\mathrm{Br}\right.$ or I) with $\mathrm{PEt}_{3}$ in benzene gave yellow $\left[\mathrm{In}_{2} \mathrm{X}_{4}\left(\mathrm{PEt}_{3}\right)_{2}\right]$, suggested to contain In-In bonds on the basis of vibrational spectroscopic data [221]. The reaction of ${ }^{n} \mathrm{Pr}_{3} \mathrm{PI}_{2}$ with indium powder in diethyl ether formed $\left[\mathrm{In}_{2} \mathrm{I}_{4}\left(\mathrm{P}^{n} \mathrm{Pr}_{3}\right)_{2}\right]$ the structure of which confirmed the presence of an unbridged In-In bond with a length of 2.745(3) ̊ [222]; curiously, $\mathrm{Ph}_{3} \mathrm{PI}_{2}$ or ${ }^{i} \mathrm{Pr}_{3} \mathrm{PI}_{2}$ gave In(III) complexes in similar reactions.

For indium(III) halides there are well established examples of four-coordinate $\left[\operatorname{InX}_{3}\left(\mathrm{PR}_{3}\right)\right]$ [122,222,225,227-229] and five-coordinate trigonal bipyramidal trans-[ $\left[\operatorname{InX}_{3}\left(\mathrm{PR}_{3}\right)_{2}\right]$ $[223,224,226,227,229]$ complexes (Fig. 35), and Table 9 contains details of those structurally characterised. There are also reports of some six-coordinate $\left[\mathrm{InX}_{3}\left(\mathrm{PR}_{3}\right)_{3}\right]$ species, but these lose $\mathrm{PR}_{3}$ very easily and there seem to be no crystallographically authenticated cases with monodentate phosphines, although six-coordination is well established with diphosphines.

The complexes are usually prepared by reaction of $\operatorname{In} X_{3}(X=C l$, $\mathrm{Br}, \mathrm{I}$ ) (there are no examples with $\mathrm{X}=\mathrm{F}[130]$ ) with the phosphine 
(a)
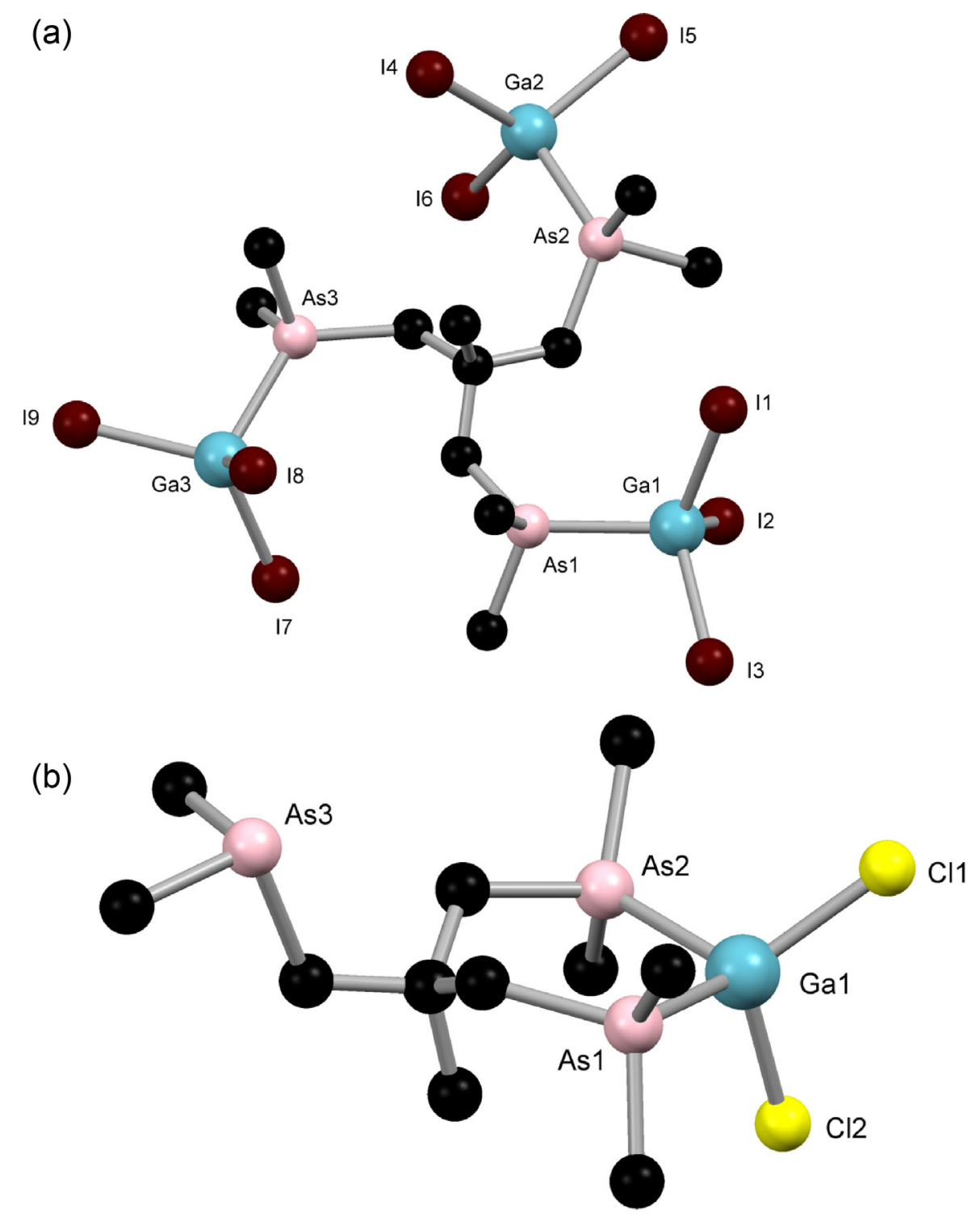

(c)

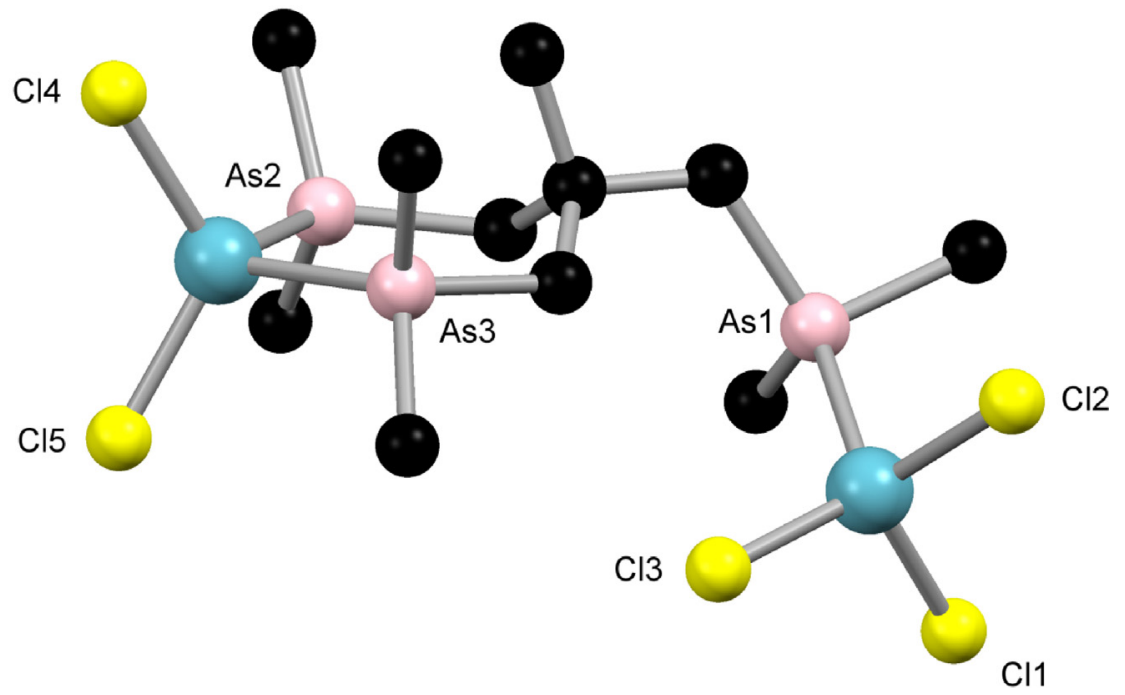

Fig. 31. (a) View of the structure of $\left[\left(\mathrm{GaI}_{3}\right)_{3}\left\{\mathrm{MeC}\left(\mathrm{CH}_{2} \mathrm{AsMe}_{2}\right)_{3}\right\}\right]$ redrawn from Ref. [182]. (b) View of the structure of the cation $\left[\mathrm{GaCl}_{2}\left\{\mathrm{MeC}\left(\mathrm{CH}_{2} \mathrm{AsMe}_{2}\right)_{3}\right\}\right]^{+}$redrawn from Ref. [182]. (c) View of the structure of the cation $\left[\left(\mathrm{GaCl}_{3}\right)\left(\mathrm{GaCl}_{2}\right)\left\{\mathrm{MeC}\left(\mathrm{CH}_{2} \mathrm{AsMe}_{2}\right)_{3}\right\}\right]^{+}$redrawn from Ref. [182]. 
Table 9

X-ray structures of indium complexes.

\begin{tabular}{|c|c|c|c|c|}
\hline Complex & In-P/As/Sb $(\AA)$ & $\operatorname{In}-X(\AA)^{\mathrm{a}}$ & Comments & Ref. \\
\hline$\left[\mathrm{In}\left(\mathrm{PPh}_{3}\right)_{3}\right]^{+}$ & $2.965(1)-3.031(1)$ & & Trig pyramidal & [185] \\
\hline$\left[\mathrm{In}\left(\mathrm{P}^{t} \mathrm{Bu}_{3}\right\}_{2}\right]\left[\mathrm{Al}\left\{\mathrm{OC}\left(\mathrm{CF}_{3}\right)_{3}\right\}_{4}\right]$ & $3.035(1), 3.055(1)$ & & v-Shaped & [185] \\
\hline$\left[\mathrm{In}_{2} \mathrm{I}_{4}\left(\mathrm{P}^{\mathrm{n}} \mathrm{Pr}_{3}\right)_{2}\right]$ & $2.585(6)$ & $2.755(3)$ & In-In dimer & [222] \\
\hline$\left[\mathrm{InCl}_{3}\left(\mathrm{PPh}_{3}\right)_{2}\right]$ & $2.6965(5), 2.7290(6)$ & $2.3907(6)-2.4055(5)$ & Tbp & [229] \\
\hline$\left[\mathrm{InBr}_{3}\left(\mathrm{PPh}_{3}\right)_{2}\right]$ & $2.742(8), 2.712(9)$ & $2.5529(4)-2.5556(4)$ & Tbp & [229] \\
\hline$\left[\mathrm{InI}_{3}\left(\mathrm{PPh}_{3}\right)_{2}\right]$ & $2.86(1), 2.99(1)$ & $2.707(1)$ & Tbp & [227] \\
\hline$\left[\mathrm{InI}_{3}\left(\mathrm{PPh}_{3}\right)\right]$ & $2.603(7)$ & $2.677(1)$ & $\mathrm{Td}$ & [227] \\
\hline$\left[\mathrm{InI}_{3}\left(\mathrm{PPh}_{3}\right)\right]$ & $2.616(9)$ & $2.691(2)$ & In co-cryst. sample & [227] \\
\hline$\left[\mathrm{InBr}_{3}\left(\mathrm{PMe}_{2} \mathrm{Ph}\right)_{2}\right]$ & $2.614(3), 2.622(3)$ & $2.565(2)-2.277(2)$ & Tbp & {$[224]$} \\
\hline$\left[\mathrm{InI}_{3}\left(\mathrm{PMePh}_{2}\right)_{2}\right]$ & $2.712(3), 2.719(3)$ & $2.7362(14)-2.776(2)$ & Tbp & {$[224]$} \\
\hline$\left[\mathrm{InI}_{3}\left(\mathrm{P}^{i} \mathrm{Pr}_{3}\right)\right]$ & $2.569(7)$ & $2.689(3)-2.705(3)$ & $\mathrm{Td}$ & [222] \\
\hline$\left[\mathrm{InI}_{3}\left\{\mathrm{P}\left(\mathrm{SiMe}_{3}\right)_{3}\right\}\right]$ & $2.537(3)$ & $2.6914(16)-2.7252(15)$ & $\mathrm{Td}$ & [225] \\
\hline$\left[\mathrm{InCl}_{3}\left(\mathrm{PMe}_{3}\right)_{2}\right]$ & $2.575(3), 2.576(3)$ & $2.453(4)-2.503(3)$ & Tbp & [226] \\
\hline$\left[\mathrm{InI}_{3}\left(\mathrm{AsPh}_{3}\right)_{2}\right]$ & $2.926(2), 2.990(2)$ & $2.7024(5)$ & Tbp & [173] \\
\hline$\left[\mathrm{InI}_{3}\left(\mathrm{AsPh}_{3}\right)\right]$ & $2.7238(13)$ & $2.6811(6)$ & $\mathrm{Td}$ & [173] \\
\hline$\left[\mathrm{InBr}_{3}\left(\mathrm{PH}^{t} \mathrm{Bu}_{2}\right)\right]$ & $2.577(2), 2.578(2)$ & $2.504(1), 2.508(1)$ & $\mathrm{Td}$ & {$[122]$} \\
\hline$\left[\operatorname{InI}_{3}\left(\mathrm{PHPh}_{2}\right)\right]$ & $2.605(9)$ & $2.672(5)-2.676(5)$ & $\mathrm{Td}$ & [228] \\
\hline$\left[\mathrm{InI}_{3}\left(\mathrm{PH}^{t} \mathrm{Bu}_{2}\right)\right]$ & $2.586(6)$ & $2.668(2)-2.679(2)$ & $\mathrm{Td}$ & [228] \\
\hline$\left[\mathrm{InCl}_{3}(\mathrm{TMPP})\right]^{\mathrm{b}}$ & $2.5281(5)$ & $2.3870(6)-2.4056(6)$ & $\mathrm{Td}$ & [229] \\
\hline$\left[\operatorname{InBr}_{3}(\mathrm{TMPP})\right]^{\mathrm{b}}$ & $2.5495(6)$ & $2.5407(3)-2.5245(3)$ & $\mathrm{Td}$ & [229] \\
\hline$\left[\mathrm{InCl}_{3}\left\{\mathrm{P}\left(o-\mathrm{MeOC}_{6} \mathrm{H}_{4}\right)_{3}\right\}\right]$ & $2.5431(5)$ & $2.3768(4)-2.3827(4)$ & $\mathrm{Td}$ & [229] \\
\hline$\left[\operatorname{InBr}_{3}\left\{\mathrm{P}\left(o-\mathrm{MeOC}_{6} \mathrm{H}_{4}\right)_{3}\right\}\right]$ & $2.5589(16)$ & $2.5118(10)-2.5231(10)$ & $\mathrm{Td}$ & [229] \\
\hline$\left[\mathrm{InCl}_{3}\left\{\mathrm{P}\left(p-\mathrm{MeOC}_{6} \mathrm{H}_{4}\right)_{3}\right\}_{2}\right]$ & $2.862(2)$ & $2.3929(18)-2.405(2)$ & Tbp & [229] \\
\hline$\left[\mathrm{InBr}_{3}\left\{\mathrm{P}\left(p-\mathrm{MeOC}_{6} \mathrm{H}_{4}\right)_{3}\right\}\right]$ & $2.5724(10)$ & $2.4835(4)$ & $\mathrm{Td}$ & [229] \\
\hline$\left[\mathrm{InI} 3\left\{\mathrm{P}\left(p-\mathrm{MeOC}_{6} \mathrm{H}_{4}\right)_{3}\right\}\right]$ & $2.5698(18)$ & $2.6841(8)-2.6912(8)$ & $\mathrm{Td}$ & [229] \\
\hline$\left[\left\{\mathrm{InI}_{3}\left\{\boldsymbol{\mu}-\mathrm{Ph}_{2} \mathrm{P}\left(\mathrm{CH}_{2}\right)_{2} \mathrm{PPh}_{2}\right\}\right\}_{\mathrm{n}}\right]$ & $2.758(6), 2.769(7)$ & $2.729(2), 2.766(3)$ & 5-Coord & [227] \\
\hline$\left[\left(\mathrm{InI}_{3}\right)_{2}\left\{\mu-\mathrm{Ph}_{2} \mathrm{P}\left(\mathrm{CH}_{2}\right)_{2} \mathrm{PPh}_{2}\right\}\right]$ & $2.6035(12), 2.5966(12)$ & $2.6523(6)-2.7032(6)$ & 4-Coord & [231] \\
\hline$\left[\left(\mathrm{InI}_{3}\right)_{2}\left\{\mathrm{Ph}_{2} \mathrm{P}\left(\mathrm{CH}_{2}\right)_{2} \mathrm{PPh}_{2}\right\}_{3}\right]$ & $2.798(3), 2.819(2)$ & $2.713(2)-2.742(2)$ & See text & [226] \\
\hline$\left[\mathrm{InI}_{3}\left\{c i s-\mathrm{Ph}_{2} \mathrm{PCH}=\mathrm{CHPPh}\right\}\right.$ & $2.5871(12)$ & $2.7003(5)-2.7420(6)$ & 4 -Coord $\kappa^{1}$ ligand & [181] \\
\hline$\left[\mathrm{InBr}_{2}\left(\text { cis- }-\mathrm{Ph}_{2} \mathrm{PCH}=\mathrm{CHPPh}_{2}\right)_{2}\right]\left[\mathrm{InBr}_{4}\right]$ & $2.696(3), 2.772(4)$ & $2.5985(1)$ & 6 -Coord & {$[181]$} \\
\hline$\left[\mathrm{InCl}_{2}\left\{0-\mathrm{C}_{6} \mathrm{H}_{4}\left(\mathrm{PPh}_{2}\right)_{2}\right\}_{2}\right]\left[\mathrm{InCl}_{4}\right]$ & $2.6648(6), 2.7133(6)$ & $2.4822(5)$ & 6-Coord & [179] \\
\hline$\left[\mathrm{InI}_{3}\left\{\mathrm{o}-\mathrm{C}_{6} \mathrm{H}_{4}\left(\mathrm{PPh}_{2}\right)_{2}\right\}\right]$ & $2.7140(9), 2.7569(9)$ & $2.7134(4)-2.7875(5)$ & 5-Coord & [179] \\
\hline$\left[\operatorname{InI}_{2}\left\{0-\mathrm{C}_{6} \mathrm{H}_{4}\left(\mathrm{PPh}_{2}\right)_{2}\right\}\right]\left[\mathrm{InI}_{4}\right]$ & $2.5862(11), 2.5635(11)$ & $2.6497(5), 2.6644(6)$ & 4-Coord & [179] \\
\hline$\left[\mathrm{InBr}_{2}\left\{\mathrm{o}-\mathrm{C}_{6} \mathrm{H}_{4}\left(\mathrm{PMe}_{2}\right)_{2}\right\}\right]\left[\mathrm{InBr}_{4}\right]$ & $2.552(3)$ & $2.4563(16), 2.4737(16)$ & 4-Coord & [232] \\
\hline$\left[\mathrm{InI}_{2}\left\{0-\mathrm{C}_{6} \mathrm{H}_{4}\left(\mathrm{PMe}_{2}\right)_{2}\right\}\right]\left[\mathrm{InI}_{4}\right]$ & $2.5579(9)$ & $2.6574(6), 2.6666(6)$ & 4-Coord & [232] \\
\hline$\left[\mathrm{InBr}_{2}\left\{0-\mathrm{C}_{6} \mathrm{H}_{4}\left(\mathrm{PMe}_{2}\right)_{2}\right\}_{2}\right]\left[\mathrm{InBr}_{4}\right]$ & $2.631(4), 2.641(4)$ & $2.641(4)$ & 6-Coord & [232] \\
\hline$\left[\mathrm{In}_{2} \mathrm{Cl}_{6}\left\{0-\mathrm{C}_{6} \mathrm{H}_{4}\left(\mathrm{PMe}_{2}\right)_{2}\right\}_{2}\right]$ & $2.6278(16), 2.6575(15)$ & $2.4389(15)-2.5721(14)$ & 6-Coord dimer & [232] \\
\hline$\left[\mathrm{In}_{2} \mathrm{Cl}_{5}\left\{\mathrm{o}-\mathrm{C}_{6} \mathrm{H}_{4}\left(\mathrm{PMe}_{2}\right)_{2}\right\}_{2}\right]\left[\mathrm{InCl}_{4}\right]$ & $2.601(5)-2.2 .614(6)$ & $2.398(6)-2.627(5)$ & Polymer see text & [232] \\
\hline \multicolumn{5}{|c|}{$\left[\operatorname{InI}_{2}\left\{0-\mathrm{C}_{6} \mathrm{H}_{4}\left(\mathrm{PMe}_{2}\right)_{2}\right\}_{2}\right]\left[\operatorname{InI}_{4}\left\{\mathrm{o}-\mathrm{C}_{6} \mathrm{H}_{4}\left(\mathrm{PMe}_{2}\right)_{2}\right\}\right]$} \\
\hline Cation & $2.6384(16)$ & $2.9124(6)$ & 6-Coord & \\
\hline Anion & $2.6886(18)$ & $2.7923(7), 2.9362(5)$ & 6-Coord & [232] \\
\hline$\left[\mathrm{In}_{2} \mathrm{Cl}_{6}\left\{o-\mathrm{C}_{6} \mathrm{H}_{4}\left(\mathrm{AsMe}_{2}\right)_{2}\right\}_{2}\right]$ & $2.7134(12), 2.7582(12)$ & $2.423(2)-2.648(2)$ & 6-Coord dimer & [232] \\
\hline$\left[\mathrm{InI}_{2}\left\{0-\mathrm{C}_{6} \mathrm{H}_{4}\left(\mathrm{AsMe}_{2}\right)_{2}\right\}\right]\left[\mathrm{InI}_{4}\right]$ & $2.6400(5)$ & $2.6472(7), 2.6603(6)$ & 4-Coord & [232] \\
\hline$\left[\mathrm{In}_{2} \mathrm{Cl}_{5}\left\{o-\mathrm{C}_{6} \mathrm{H}_{4}\left(\mathrm{PMe}_{2}\right)_{2}\right\}_{2}\right]\left[\mathrm{InCl}_{4}\right]$ & $2.6891(1), 2.7039(10)$ & $2.3859(10)-2.6150(7)$ & Polymer see text & [232] \\
\hline$\left[\mathrm{InCl}_{2}\left\{\mathrm{PhP}\left(o-\mathrm{C}_{6} \mathrm{H}_{4} \mathrm{PPh}_{2}\right)_{2}\right\}\right]\left[\mathrm{InCl}_{4}\right]$ & $2.5956(8), 2.5799(7)$ & $2.3547(9), 2.4172(8)$ & 4-Coord & [233] \\
\hline$\left[\mathrm{InI}_{2}\left\{\mathrm{PhP}\left(0-\mathrm{C}_{6} \mathrm{H}_{4} \mathrm{PPh}_{2}\right)_{2}\right\}\right]\left[\mathrm{InI}_{4}\right]$ & $2.5982(10), 2.6239(10)$ & $2.7092(5), 2.7376(5)$ & 4-Coord & [179] \\
\hline$\left[\mathrm{InH}_{3}\left(\mathrm{PC}_{3}\right)\right]$ & $2.6474(6)$ & & $\mathrm{Td}$ & [234] \\
\hline$\left[\mathrm{InH}_{3}\left(\mathrm{PCy}_{3}\right)_{2}\right]$ & $2.9869(5)$ & & Tbp & {$[235]$} \\
\hline$\left[\operatorname{In}(\mathrm{SPh})_{3}\left(\mathrm{PCy}_{3}\right)\right]$ & $2.6109(8)$ & & $\mathrm{Td}$ & [235] \\
\hline$\left[\mathrm{In}(\mathrm{SePh})_{3}\left(\mathrm{PCy}_{3}\right)\right]$ & $2.6145(11)$ & & $\mathrm{Td}$ & [235] \\
\hline$\left[\mathrm{In}(\mathrm{TePh})_{3}\left(\mathrm{PCy}_{3}\right)\right]$ & $2.642(3)$ & & $\mathrm{Td}$ & [235] \\
\hline$\left[\mathrm{In}(\mathrm{SPh})_{3}\left(\mathrm{PPh}_{3}\right)\right]$ & $2.6458(12)$ & & $\mathrm{Td}$ & [243] \\
\hline$\left[\operatorname{InMe}_{3}\left(\mathrm{PPh}_{3}\right)\right]$ & $2.733(2)$ & & $\mathrm{Td}$ & [141] \\
\hline$\left[\mathrm{InEt}_{3}\left(\mathrm{PPh}_{3}\right)\right]$ & $2.7453(5)$ & & $\mathrm{Td}$ & [141] \\
\hline$\left[\mathrm{In}\left(\mathrm{Me}_{3} \mathrm{CCH}_{2}\right)_{3}\left\{\mathrm{P}\left(\mathrm{SiMe}_{3}\right)_{3}\right\}\right]$ & $2.806(4)-2.944(4)$ & & $\mathrm{Td}$ & [237] \\
\hline$\left[\mathrm{In}\left(\mathrm{Me}_{3} \mathrm{CCH}_{2}\right)_{3}\left\{\mathrm{As}\left(\mathrm{SiMe}_{3}\right)_{3}\right\}\right]$ & $2.888(2)-3.018(1)$ & & $\mathrm{Td}$ & [237] \\
\hline$\left[\mathrm{InMe}\left(\mathrm{Me}_{3} \mathrm{CCH}_{2}\right)_{2}\left\{\mathrm{P}\left(\mathrm{SiMe}_{3}\right)_{3}\right\}\right]$ & $2.796(3), 2.802(3)$ & & $\mathrm{Td}$ & [237] \\
\hline$\left[\mathrm{In}\left(\mathrm{Me}_{3} \mathrm{SiCH}_{2}\right)_{3}\left\{\mathrm{As}\left(\mathrm{SiMe}_{3}\right)_{3}\right\}\right]$ & $2.778(4)$ & & $\mathrm{Td}$ & [201] \\
\hline$\left[\mathrm{In}\left(\mathrm{Me}_{3} \mathrm{SiCH}_{2}\right)_{3}\left\{\mathrm{Sb}\left(\mathrm{SiMe}_{3}\right)_{3}\right\}\right]$ & $3.0078(6)$ & & $\mathrm{Td}$ & [190] \\
\hline$\left[\left(\mathrm{InMe}_{3}\right)_{2}\left(\mu-\mathrm{Ph}_{2} \mathrm{P}\left(\mathrm{CH}_{2}\right)_{2} \mathrm{PPh}_{2}\right)\right]$ & $2.755(4)$ & & Dimer & [146] \\
\hline$\left[\left(\mathrm{InMe}_{3}\right)_{2}\left(\mu-\mathrm{Ph}_{2} \mathrm{P}\left(\mathrm{CH}_{2}\right)_{3} \mathrm{PPh}_{2}\right)\right]$ & $2.7043(5)$ & & Dimer & [141] \\
\hline$\left[\left(\mathrm{InEt}_{3}\right)_{2}\left(\mu-\mathrm{Ph}_{2} \mathrm{P}\left(\mathrm{CH}_{2}\right)_{3} \mathrm{PPh}_{2}\right)\right]$ & $2.7468(6)$ & & Dimer & {$[141]$} \\
\hline$\left[\operatorname{In}\left(\sigma-\mathrm{C}_{5} \mathrm{H}_{5}\right)_{3} \mathrm{PPh}_{3}\right]$ & $2.6992(4)$ & & $\mathrm{Td}$ & [239] \\
\hline$\left[\mathrm{InI}_{2}\left(\mathrm{CH}_{2} \mathrm{C}_{6} \mathrm{H}_{9}\right) \mathrm{PPh}_{3}\right]$ & $2.6341(14)$ & $2.7189(8), 2.7252(8)$ & $\mathrm{Td}$ & [241] \\
\hline$\left[\mathrm{In}\left(\mathrm{Ph}_{2} \mathrm{PCH}_{2} \mathrm{C}_{6} \mathrm{H}_{4}\right)_{3}\right]$ & $2.845(1), 2.925(1)$ & & 5-Coord $\mathrm{C}_{3} \mathrm{P}_{2}$ & {$[162]$} \\
\hline$\left[\mathrm{InCl}_{2}\left\{{ }^{t} \mathrm{Bu}_{2} \mathrm{PCH}_{2} \mathrm{CH}_{2} \mathrm{C}_{6} \mathrm{H}_{4}\right\}\right]$ & $2.595(2)$ & & 4-Coord & [215] \\
\hline$\left[\mathrm{InCl}(\mathrm{CB})_{2}\right]^{\mathrm{C}}$ & $2.733(1)$ & & & [165] \\
\hline$\left[\mathrm{In}\left(\mathrm{Ph}_{2} \mathrm{PC}_{6} \mathrm{H}_{4} \mathrm{~S}\right)_{3}\right]$ & $2.728(2)-2.963(2)$ & & & [244] \\
\hline$\left[\mathrm{InBr}_{2}(\mathrm{III})\right]^{\mathrm{d}}$ & $2.6383(7), 2.6652(7)$ & & & [217] \\
\hline$\left[\mathrm{InI}_{2}(\mathrm{III})\right]^{\mathrm{d}}$ & $2.6994(8)-2.7451(8)$ & & & [217] \\
\hline
\end{tabular}

a Values refer to pnictogen coordinated fragment; data for $\left[\operatorname{In} \mathrm{X}_{4}\right]^{-}$are not given.

b TMPP is tris(2,4,6-trimethoxyphenyl)phosphine.

c HCB is the carbaborane (see text).

d III is the amidodiphosphine pincer. 


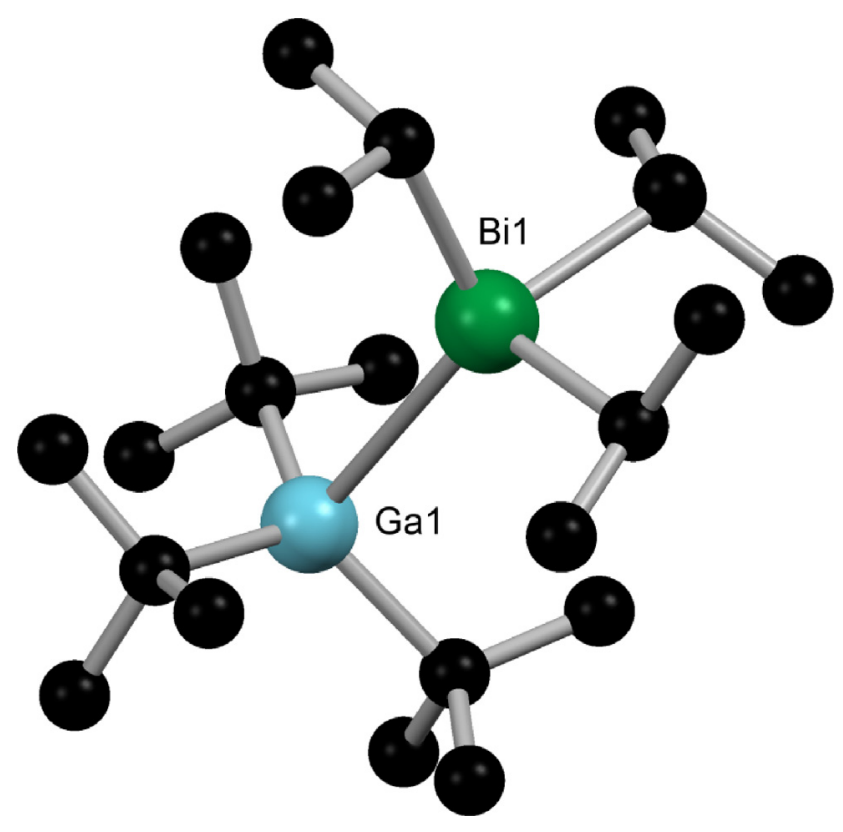

Fig. 32. View of the structure of $\left[\mathrm{Ga}^{t} \mathrm{Bu}_{3}\left(\mathrm{Bi}^{i} \mathrm{Pr}_{3}\right)\right]$ redrawn from Ref. [200].

in diethyl ether, benzene etc., although reaction of indium powder with $\mathrm{R}_{3} \mathrm{PI}_{2}$ [222] has also been used for some iodo-complexes. The complexes isolated depend upon the $\mathrm{PR}_{3} / \mathrm{X}$ combination present and rather less on the reaction conditions. For a common (or similar) ligand, the same trends in In-E bond lengths are found as the halide is changed as discussed previously for $\mathrm{Al}$ and $\mathrm{Ga}$ analogues. Comparisons between the four- and five-coordinate complexes can also be made, especially for $\mathrm{PPh}_{3}$ and $\mathrm{AsPh}_{3}$ where both forms have been found co-crystallised in the same samples $[173,222,227]$. Here the axial In-P(As) bonds in the five-coordinate trigonal bipyramidal complexes are much longer than those in the four-coordinate tetrahedral forms, and notably the differences are

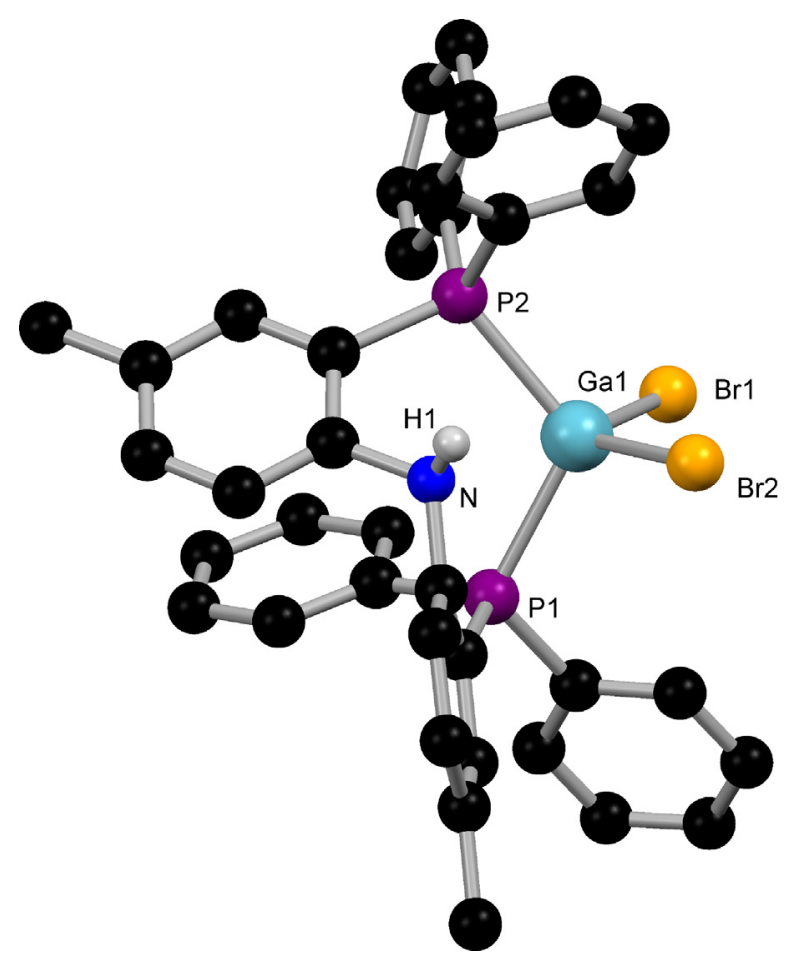

Fig. 33. View of the structure of the cation $\left[\mathrm{GaBr}_{2}(\mathbf{I I I}-\mathbf{H})\right]^{+}$redrawn from Ref. [217].

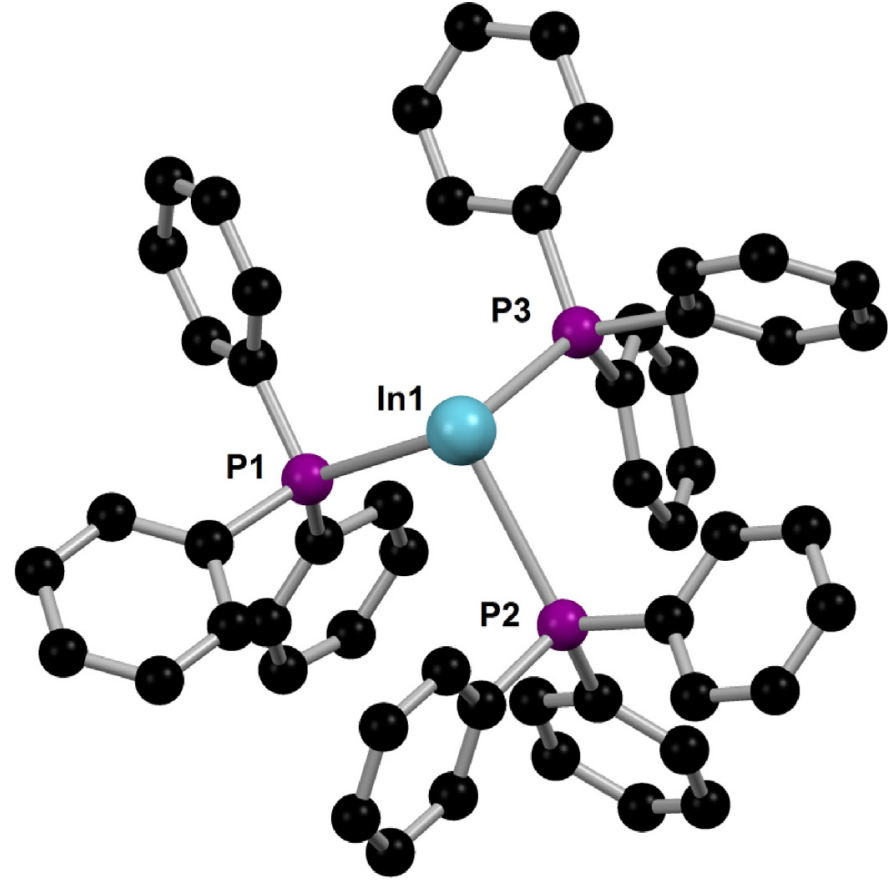

Fig. 34. View of the structure of the cation $\left[\operatorname{In}\left(\mathrm{PPh}_{3}\right)_{3}\right]^{+}$redrawn from Ref. [185].

greater than between In-X in the two structure types. This indicates that the axial $\mathrm{ER}_{3}$ groups are quite weakly bound in the former complexes, and correlates with the easy loss of $\mathrm{ER}_{3}$ in solution. Two caveats to this observation are, (a) the axial $\mathrm{P}($ As) $-\mathrm{In}-\mathrm{P}(\mathrm{As})$ are $3 \mathrm{c}-4 \mathrm{e}$ bonds compared with $2 \mathrm{c}-2 \mathrm{e}$ bonds for In-I in both forms, or for In-P(As) in the tetrahedral complex, and (b) the dimensions in $\left[\operatorname{InI}_{3}\left(\mathrm{PPh}_{3}\right)\right]$ in a crystal of the pure complex exhibit small but significant differences from those in the same molecule in the co-crystallised $\left[\operatorname{InI}_{3}\left(\mathrm{PPh}_{3}\right)\right] \cdot\left[\mathrm{InI}_{3}\left(\mathrm{PPh}_{3}\right)_{2}\right]$, which suggests crystal packing also affects the bond lengths in these systems. Solution spectroscopic studies have been carried out on some systems to probe the four- $\leftrightarrow$ five-coordinate interconversions using ${ }^{31} \mathrm{P}\left\{{ }^{1} \mathrm{H}\right\}$

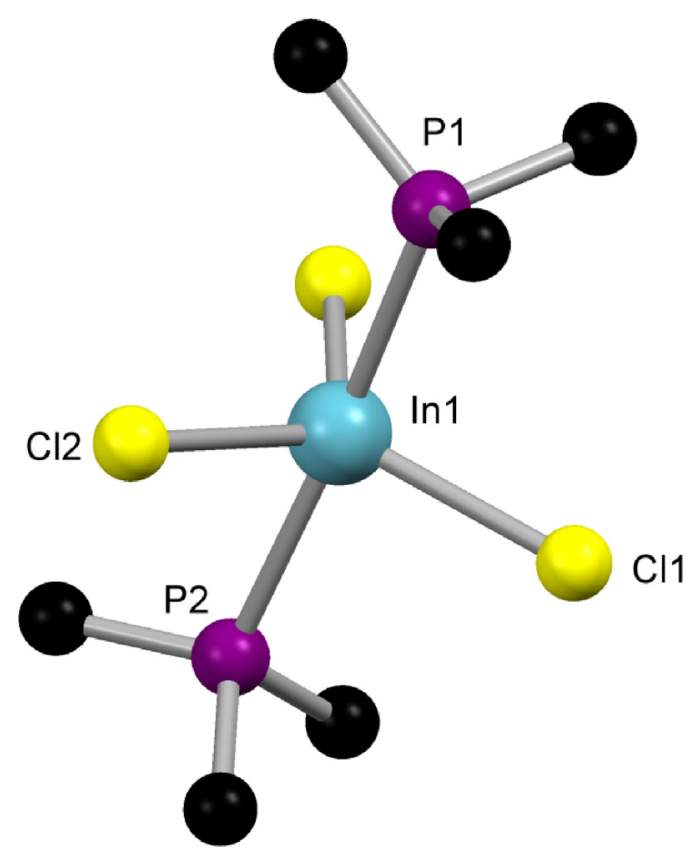

Fig. 35. View of the structure of $\left[\mathrm{InCl}_{3}\left(\mathrm{PMe}_{3}\right)_{2}\right]$ redrawn from Ref. [226]. 


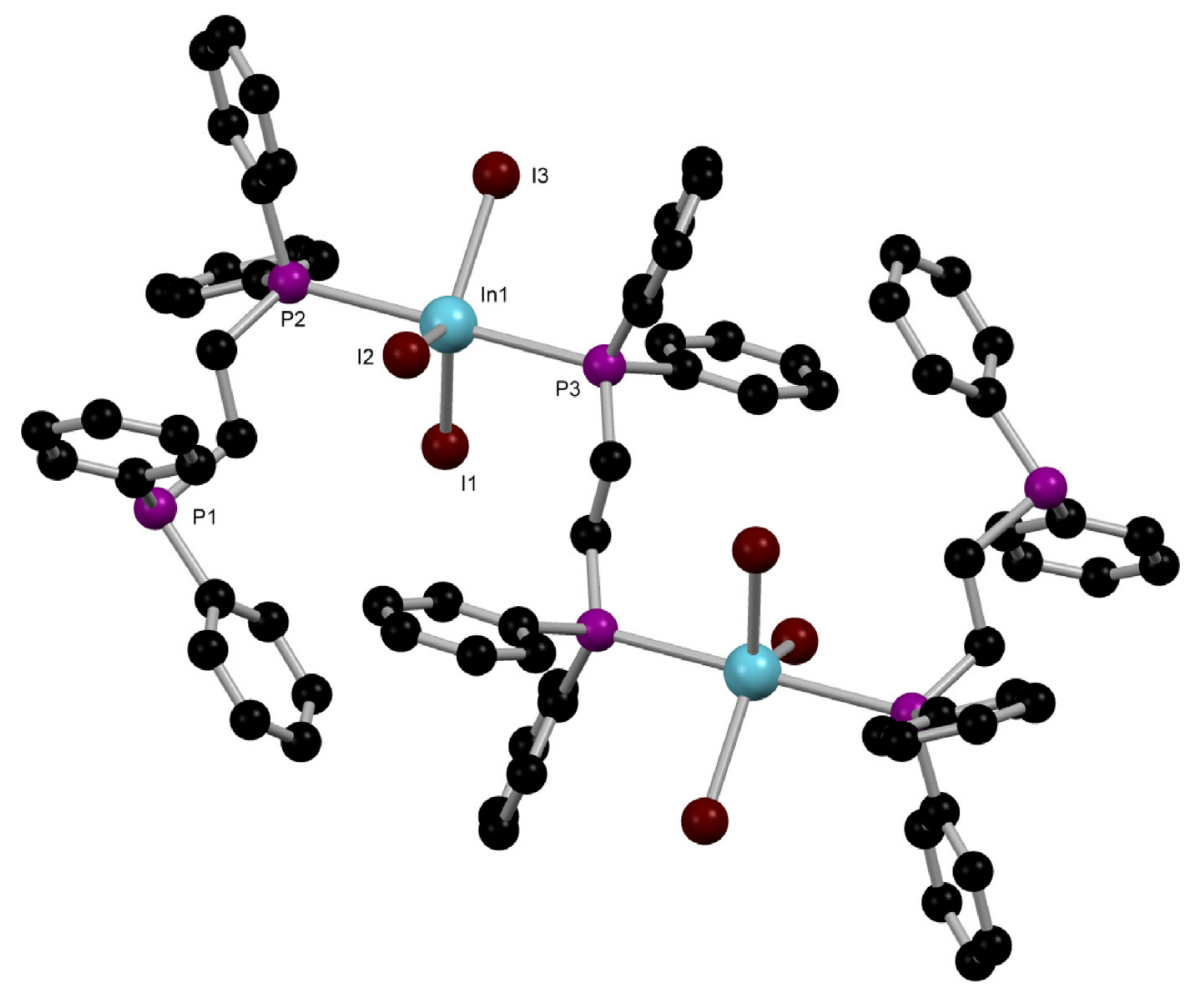

Fig. 36. View of the structure of $\left[\left(\mathrm{InI}_{3}\right)_{2}\left\{\mathrm{Ph}_{2} \mathrm{P}\left(\mathrm{CH}_{2}\right)_{2} \mathrm{PPh}_{2}\right\}_{3}\right]$ redrawn from Ref. [226].

and ${ }^{115}$ In NMR spectroscopy $[227,228]$. The substantial quadrupole moment of ${ }^{115}$ In results in very broad lines and thus it is correspondingly of limited use, but the data are consistent with fast phosphine ligand exchange in solution down to low temperatures in all the systems studied, and extensive dissociation of phosphine in the five-coordinate complexes. Notably, the ${ }^{31} \mathrm{P}\left\{{ }^{1} \mathrm{H}\right\}$ NMR coordination shifts are mostly negative for indium-coordinated phosphines (Table 8), although a theoretical explanation of this effect is lacking. ${ }^{115}$ In NMR spectra have also been obtained from a range of solid indium(III) halide-phosphine adducts [229,230]. As for $\mathrm{Al}$ and $\mathrm{Ga}$, there are no reports of successful isolation of indium halide-stibine complexes.

Indium(III) halide complexes with diphosphines show a greater variety of structures compared with those of the lighter elements. For example, complexes with $\mathrm{InI}_{3}: \mathrm{Ph}_{2} \mathrm{P}\left(\mathrm{CH}_{2}\right)_{2} \mathrm{PPh}_{2}$ ratios of $1: 1,2: 1$ and $2: 3$ have been isolated and their structures determined [226,227,231]. The $1: 1$ adduct is a chain polymer with five-coordinate indium and bridging diphosphines, whilst the 2:1 complex is a discrete dimer with four-coordinate In and a single bridging diphosphine. The $2: 3$ complex is also a dimer with a single diphosphine bridge between two five-coordinate indium centres, each further coordinated to $\kappa^{1}$-diphosphines (Fig. 36). However, in $\left[\operatorname{InI}_{3}\left\{c i s-\mathrm{Ph}_{2} \mathrm{PCH}=\mathrm{CHPPh}_{2}\right\}\right]$ a discrete distorted tetrahedral complex is present with the diphosphine bound as a monodentate ligand, whilst the $1: 1$ complex with $\mathrm{InBr}_{3}$ is ionic trans-[ $\left.\operatorname{InBr}_{2}\left(\text { cis- }-\mathrm{Ph}_{2} \mathrm{PCH}=\mathrm{CHPPh}_{2}\right)_{2}\right]\left[\mathrm{InBr}_{4}\right][181]$. In contrast to gallium(III), indium(III) does not cause cis $\rightarrow$ trans isomerisation of this diphosphine [181]. The stronger $\sigma$-donor $\mathrm{Et}_{2} \mathrm{P}\left(\mathrm{CH}_{2}\right)_{2} \mathrm{PEt}_{2}$ gave $1: 1$ complexes with $\operatorname{In} \mathrm{X}_{3}$, but since the vibrational spectra showed the absence of $\left[\operatorname{InX}_{4}\right]^{-}$ions, these are probably dimers $\left[\mathrm{In}_{2} \mathrm{X}_{6}\left\{\mathrm{Et}_{2} \mathrm{P}\left(\mathrm{CH}_{2}\right)_{2} \mathrm{PEt}_{2}\right\}_{2}\right][232]$.

The rigid $o$-phenylene diphosphines behave differently to the flexible diphosphinoalkanes. Indium trichloride and $o-\mathrm{C}_{6} \mathrm{H}_{4}\left(\mathrm{PPh}_{2}\right)_{2}$ produced the six-coordinate cation trans-[ $\operatorname{InCl}_{2}\{0-$ $\left.\left.\mathrm{C}_{6} \mathrm{H}_{4}\left(\mathrm{PPh}_{2}\right)_{2}\right\}_{2}\right]\left[\mathrm{InCl}_{4}\right]$ irrespective of the ratio of reagents used (although there is NMR evidence for other species in $\mathrm{CH}_{2} \mathrm{Cl}_{2}$ solution in the presence of excess diphosphine, these could not be isolated as solids [232]), but with $\operatorname{InX}_{3}(\mathrm{X}=\mathrm{Br}$ or I) in a 1:1 ratio, the products are neutral five-coordinate distorted square pyramidal [ $\left.\operatorname{InX}_{3}\left\{o-\mathrm{C}_{6} \mathrm{H}_{4}\left(\mathrm{PPh}_{2}\right\}_{2}\right\}\right]$. From reaction in a $2: 1$ ratio the products are $\left[\operatorname{InX}_{2}\left\{0-\mathrm{C}_{6} \mathrm{H}_{4}\left(\mathrm{PPh}_{2}\right)_{2}\right\}\right]\left[\mathrm{InX}_{4}\right]$ containing tetrahedral cations [179].

Even greater complexity is found in the $\operatorname{InX} \mathrm{X}_{3} / 0-\mathrm{C}_{6} \mathrm{H}_{4}\left(\mathrm{PMe}_{2}\right)_{2}$ systems [232]. A 2:1 InX $\mathrm{I}_{3}$ to $o-\mathrm{C}_{6} \mathrm{H}_{4}\left(\mathrm{PMe}_{2}\right)_{2}$ ratio affords [ $\operatorname{InX}_{2}\{0$ $\left.\left.\mathrm{C}_{6} \mathrm{H}_{4}\left(\mathrm{PMe}_{2}\right)_{2}\right\}\right]\left[\mathrm{InX}_{4}\right]$ for all three halides, and X-ray structures (Fig. 37) show very distorted tetrahedral cations, while multinuclear NMR $\left({ }^{31} \mathrm{P}\left\{{ }^{1} \mathrm{H}\right\},{ }^{115} \mathrm{In},{ }^{1} \mathrm{H}\right)$ and IR/Raman data show these are the only forms present in the bulk solid and in chlorocarbon solutions. However, from $1: 1$ or $1: 2$ reactant ratios the products are more diverse; for $\mathrm{X}=\mathrm{Cl}$ or $\mathrm{Br}$ the products have a 1:1 stoichiometry but for $\operatorname{InI}_{3}$ the ratio of In:diphosphine is 2:3. The crystal structure for the chloro-complex showed it to be a halide-bridged dimer $\left[\mathrm{In}_{2} \mathrm{Cl}_{6}\left\{0-\mathrm{C}_{6} \mathrm{H}_{4}\left(\mathrm{PMe}_{2}\right)_{2}\right\}_{2}\right]$ (Fig. 38), whereas the bromide is ionic trans-[ $\left.\operatorname{InBr}_{2}\left\{0-\mathrm{C}_{6} \mathrm{H}_{4}\left(\mathrm{PMe}_{2}\right)_{2}\right\}_{2}\right]\left[\mathrm{InBr}_{4}\right]$. The bromide complex retains this structure in $\mathrm{CH}_{2} \mathrm{Cl}_{2}$ solution on the basis of NMR spectroscopic evidence, but the chloride partially rearranges into a mixture of the neutral dimer, $\left[\operatorname{InCl}_{2}\{0\right.$ $\left.\left.\mathrm{C}_{6} \mathrm{H}_{4}\left(\mathrm{PMe}_{2}\right)_{2}\right\}_{2}\right]\left[\mathrm{InCl}_{4}\right]$ and possibly $\left[\mathrm{InCl}_{3}\left\{0-\mathrm{C}_{6} \mathrm{H}_{4}\left(\mathrm{PMe}_{2}\right)_{2}\right\}\right]$ (but not $\left.\left[\mathrm{InCl}_{2}\left\{\mathrm{o}-\mathrm{C}_{6} \mathrm{H}_{4}\left(\mathrm{PMe}_{2}\right)_{2}\right\}\right]^{+}\right)$. A small number of crystals deposited from a $\mathrm{CH}_{2} \mathrm{Cl}_{2}$ solution of $\left[\mathrm{In}_{2} \mathrm{Cl}_{6}\left\{0-\mathrm{C}_{6} \mathrm{H}_{4}\left(\mathrm{PMe}_{2}\right)_{2}\right\}_{2}\right]$ proved to be $\left[\mathrm{In}_{2} \mathrm{Cl}_{5}\left\{0-\mathrm{C}_{6} \mathrm{H}_{4}\left(\mathrm{PMe}_{2}\right)_{2}\right\}_{2}\right]\left[\mathrm{InCl}_{4}\right]$ which contains a polymeric cation with each indium carrying a chelating diphosphine, a terminal chlorine, and three bridging chlorines, with alternating single and double chlorine bridges along the chain (Fig. 39). The complex is formally derived by $\mathrm{InCl}_{3}$ removing a chloride from the dimer to give $\left[\mathrm{InCl}_{4}\right]^{-}$, whilst the indium retains six-coordination by forming an extra chlorine bridge. The 2:3 indium iodide complex is structurally trans-[ $\operatorname{InI}_{2}\{0-$ $\left.\left.\mathrm{C}_{6} \mathrm{H}_{4}\left(\mathrm{PMe}_{2}\right)_{2}\right\}_{2}\right]\left[\mathrm{InI}_{4}\left\{0-\mathrm{C}_{6} \mathrm{H}_{4}\left(\mathrm{PMe}_{2}\right)_{2}\right\}\right]$ containing the now familiar six-coordinate cation type, but also a extremely rare example of a six-coordinate indium haloanion. NMR studies show the anion is dissociated into $\left[\mathrm{InI}_{4}\right]^{-}$and free diphosphine in chlorocarbon 


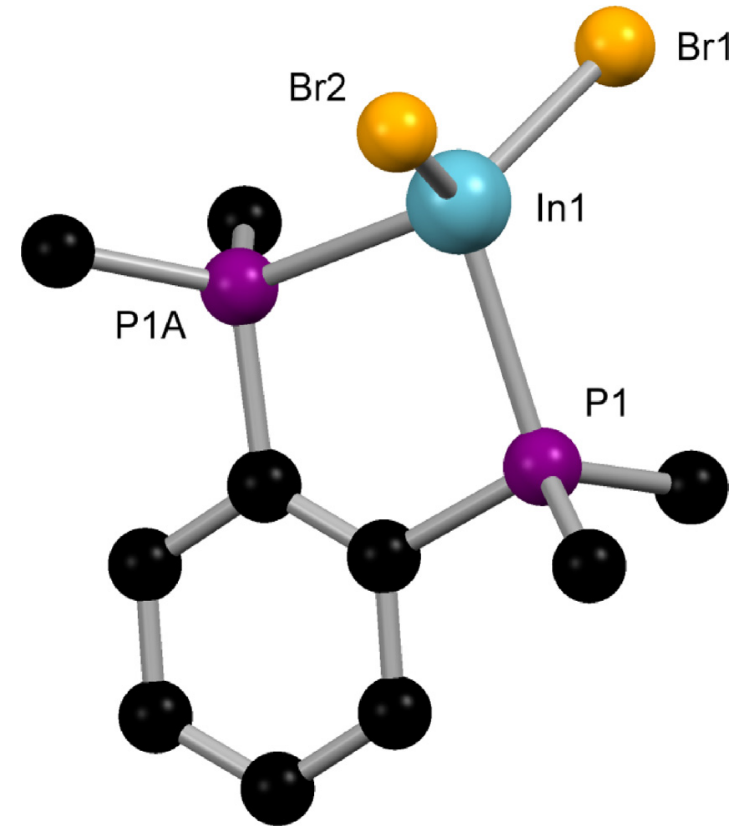

Fig. 37. View of the structure of the cation $\left[\operatorname{InBr}_{2}\left\{0-\mathrm{C}_{6} \mathrm{H}_{4}\left(\mathrm{PMe}_{2}\right)_{2}\right\}\right]^{+}$redrawn from ref. [232].

solution. The formation of the distorted octahedral anion is not explained by increased Lewis acidity of $\mathrm{InI}_{3}$, indeed the structural data suggest the iodide is the weakest Lewis acid of the three halides, but it may be due to a smaller reorganisation energy needed to convert the tetrahedral $\mathrm{InI}_{4}$ unit to the fragment needed in sixcoordination. The complexity of the $\mathrm{InX}_{3} / o-\mathrm{C}_{6} \mathrm{H}_{4}\left(\mathrm{PMe}_{2}\right)_{2}$ systems compared with the gallium systems is a result of the larger size of indium, making five- and six-coordination more favourable, but it also may reflect weaker binding of the neutral ligand, which leads to less discrimination allowing a range of structures. Indium halidephosphine complexes are moisture sensitive, with water displacing the phosphines, but in contrast to the gallium analogues, this does not generate protonated phosphines in the process [181,231,232].

The corresponding reactions [232] with the diarsine 0 $\mathrm{C}_{6} \mathrm{H}_{4}\left(\mathrm{AsMe}_{2}\right)_{2}$ were generally similar, although the $1: 1$ adducts are neutral dimers $\left[\operatorname{In}_{2} \mathrm{X}_{6}\left\{0-\mathrm{C}_{6} \mathrm{H}_{4}\left(\mathrm{AsMe}_{2}\right)_{2}\right\}_{2}\right]$ in the solid state for both $\mathrm{X}=\mathrm{Cl}$ and $\mathrm{Br}$; again partial rearrangement into [ $\operatorname{InX}_{2}\{0$ $\left.\left.\mathrm{C}_{6} \mathrm{H}_{4}\left(\mathrm{AsMe}_{2}\right)_{2}\right\}_{2}\right]^{+}$and $\left[\mathrm{InX}_{3}\left\{0-\mathrm{C}_{6} \mathrm{H}_{4}\left(\mathrm{AsMe}_{2}\right)_{2}\right\}\right]$ occurs in solution. The complexes formed from a 2:1 ratio of reagents are $\left[\operatorname{InX}_{2}\{0-\right.$ $\left.\left.\mathrm{C}_{6} \mathrm{H}_{4}\left(\mathrm{AsMe}_{2}\right)_{2}\right\}\right]\left[\mathrm{InX} \mathrm{X}_{4}\right]$ with distorted tetrahedral cations. The exact analogues, $\left[\mathrm{In}_{2} \mathrm{Cl}_{5}\left\{0-\mathrm{C}_{6} \mathrm{H}_{4}\left(\mathrm{AsMe}_{2}\right)_{2}\right\}_{2}\right]\left[\mathrm{InCl}_{4}\right]$ and trans-[ $\mathrm{InI}_{2}\{0-$ $\left.\left.\mathrm{C}_{6} \mathrm{H}_{4}\left(\mathrm{AsMe}_{2}\right)_{2}\right\}_{2}\right]\left[\mathrm{InI}_{4}\left\{0-\mathrm{C}_{6} \mathrm{H}_{4}\left(\mathrm{AsMe}_{2}\right)_{2}\right\}\right]$ of the diphosphine complexes described above were also characterised.

The triphosphine, $\mathrm{PhP}\left(0-\mathrm{C}_{6} \mathrm{H}_{4} \mathrm{PPh}_{2}\right)_{2}$, coordinates as a bidentate ligand via the terminal diphenylphosphino groups in $\left[\operatorname{InX}_{2}\{\mathrm{PhP}(o-\right.$ $\left.\left.\left.\mathrm{C}_{6} \mathrm{H}_{4} \mathrm{PPh}_{2}\right)_{2}\right\}\right]\left[\mathrm{InX} \mathrm{X}_{4}\right](\mathrm{X}=\mathrm{Cl}$ or I) $[179,233]$.

In contrast to the aluminium and gallium complexes, phosphine complexes of indium hydride cannot be made from $\mathrm{Li}\left[\mathrm{InH}_{4}\right]$, $\mathrm{HCl}$ and the appropriate phosphine [234,235]. Displacement of $\mathrm{NMe}_{3}$ from $\left[\mathrm{InH}_{3}\left(\mathrm{NMe}_{3}\right)\right]$ was successful in producing $\left[\mathrm{InH}_{3}\left(\mathrm{PR}_{3}\right)\right]$ with $\mathrm{PCy}_{3}, \mathrm{P}(\text { cyclopentyl })_{3}$ and $\mathrm{PPh}_{3}$, but failed with $\mathrm{PEt}_{3}, \mathrm{P}^{t} \mathrm{Bu}_{3}$ or $\mathrm{P}(\text { mesityl })_{3}$, which shows that steric protection is not the only requirement; electronic factors and possibly low solubility in the reaction solution may also be factors in successful isolation. Using excess $\mathrm{PC}_{3}$ in the reaction produced the five-coordinate [ $\left.\mathrm{InH}_{3}\left(\mathrm{PCy}_{3}\right)_{2}\right]$ [235], and with $\mathrm{Cy}_{2} \mathrm{P}\left(\mathrm{CH}_{2}\right)_{2} \mathrm{PCy}_{2}$ the product is polymeric $\left[\mathrm{InH}_{3}\left\{\mathrm{Cy}_{2} \mathrm{P}\left(\mathrm{CH}_{2}\right)_{2} \mathrm{PCy}_{2}\right\}\right]_{\mathrm{n}}$ [236]. [ $\left.\mathrm{InH}_{3}\left(\mathrm{PCy}_{3}\right)\right]$ reacts with $\mathrm{Ph}_{2} \mathrm{E}_{2}^{\prime}\left(\mathrm{E}^{\prime}=\mathrm{S}\right.$, Se or Te $)$ to form $\left[\mathrm{In}\left(\mathrm{E}^{\prime} \mathrm{Ph}\right)_{3}\left(\mathrm{PCy}_{3}\right)\right]$ and the structures of all three have been determined [235].

Pnictogen ligand adducts of trialkylindiums are generally similar to their lighter analogues, although less extensively studied [141,146,149,190,196,237-241]. All are distorted tetrahedral and even with tri-and tetra-dentate phosphines only one phosphine donor group coordinates to each indium centre [146].

The structure of $\left[\mathrm{In}\left(\mathrm{Me}_{3} \mathrm{SiCH}_{2}\right)_{3}\left\{\mathrm{As}\left(\mathrm{SiMe}_{3}\right)_{3}\right\}\right]$ appears unexceptional [201], but those of [ $\left.\operatorname{In}\left(\mathrm{Me}_{3} \mathrm{CCH}_{2}\right)_{3}\left\{\mathrm{E}\left(\mathrm{SiMe}_{3}\right)_{3}\right\}\right](\mathrm{E}=\mathrm{P}$ or As) each contain three independent molecules, with significant variations in the structural parameters between them ascribed to the effects of steric crowding [237]. The weaker In-C bonds also produce some differences from the chemistry of the lighter analogues. For example addition of $\left[\mathrm{InCl}\left(\mathrm{Me}_{3} \mathrm{CCH}_{2}\right)_{2}\right]$ to $\mathrm{Li}\left[\mathrm{P}\left(\mathrm{SiMe}_{3}\right)_{2}\right]$ produced [ $\left.\mathrm{InMe}\left(\mathrm{Me}_{3} \mathrm{CCH}_{2}\right)_{2}\left\{\mathrm{P}\left(\mathrm{SiMe}_{3}\right)_{3}\right\}\right]$ rather than the expected phosphido complex [237], and attempts to make the $\mathrm{PHPh}_{2}$ complex of $\left[\mathrm{In}\left(\mathrm{Me}_{3} \mathrm{CCH}_{2}\right)_{3}\right]$ resulted in formation of [ In $\left(\mathrm{Me}_{3} \mathrm{CCH}_{2}\right)_{2} \mathrm{PPh}_{2}$ ] and $\mathrm{Me}_{4} \mathrm{C}$ [196]. Few stibine adducts of $\mathrm{InR}_{3}$ have been described [149,190,238].

There are also examples of alkyls or aryls with pendant phosphino-groups. In the (diphenylphosphinomethyl) phenyl complex $\left[\operatorname{In}\left(\mathrm{Ph}_{2} \mathrm{PCH}_{2} \mathrm{C}_{6} \mathrm{H}_{4}\right)_{3}\right]$, the geometry is trigonal

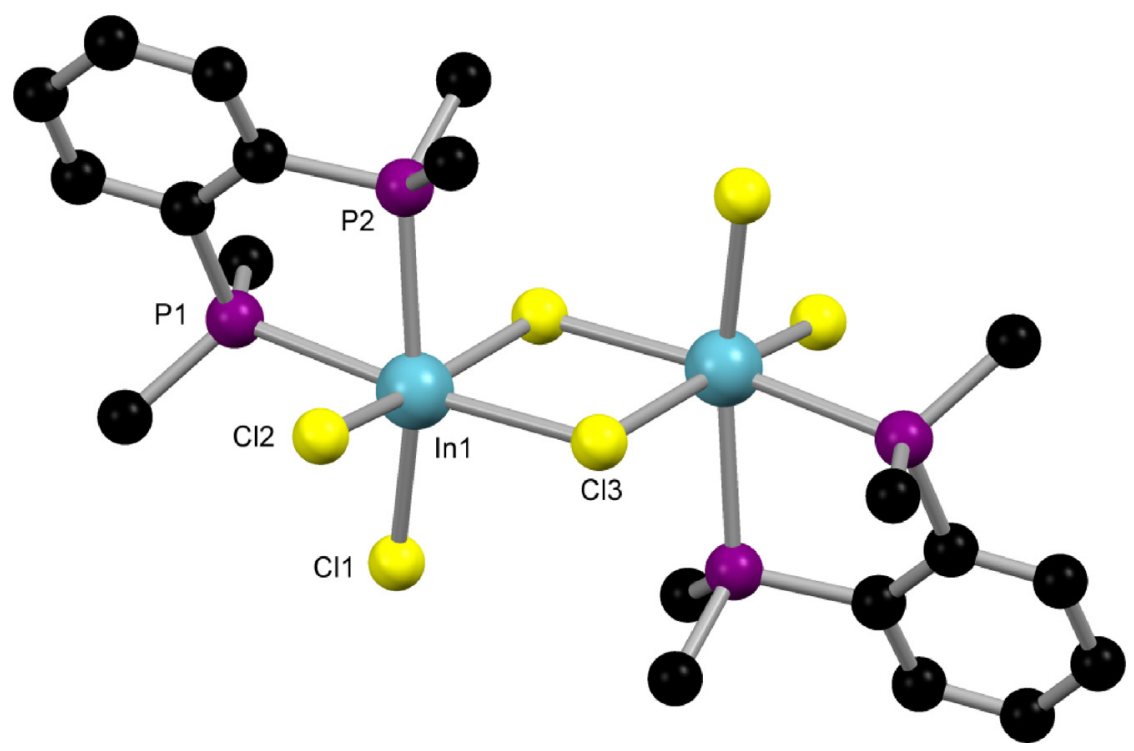

Fig. 38. View of the structure of $\left[\operatorname{In}_{2} \mathrm{Cl}_{4}(\mu-\mathrm{Cl})_{2}\left\{o-\mathrm{C}_{6} \mathrm{H}_{4}\left(\mathrm{PMe}_{2}\right)_{2}\right\}_{2}\right]$ redrawn from Ref. [232]. 


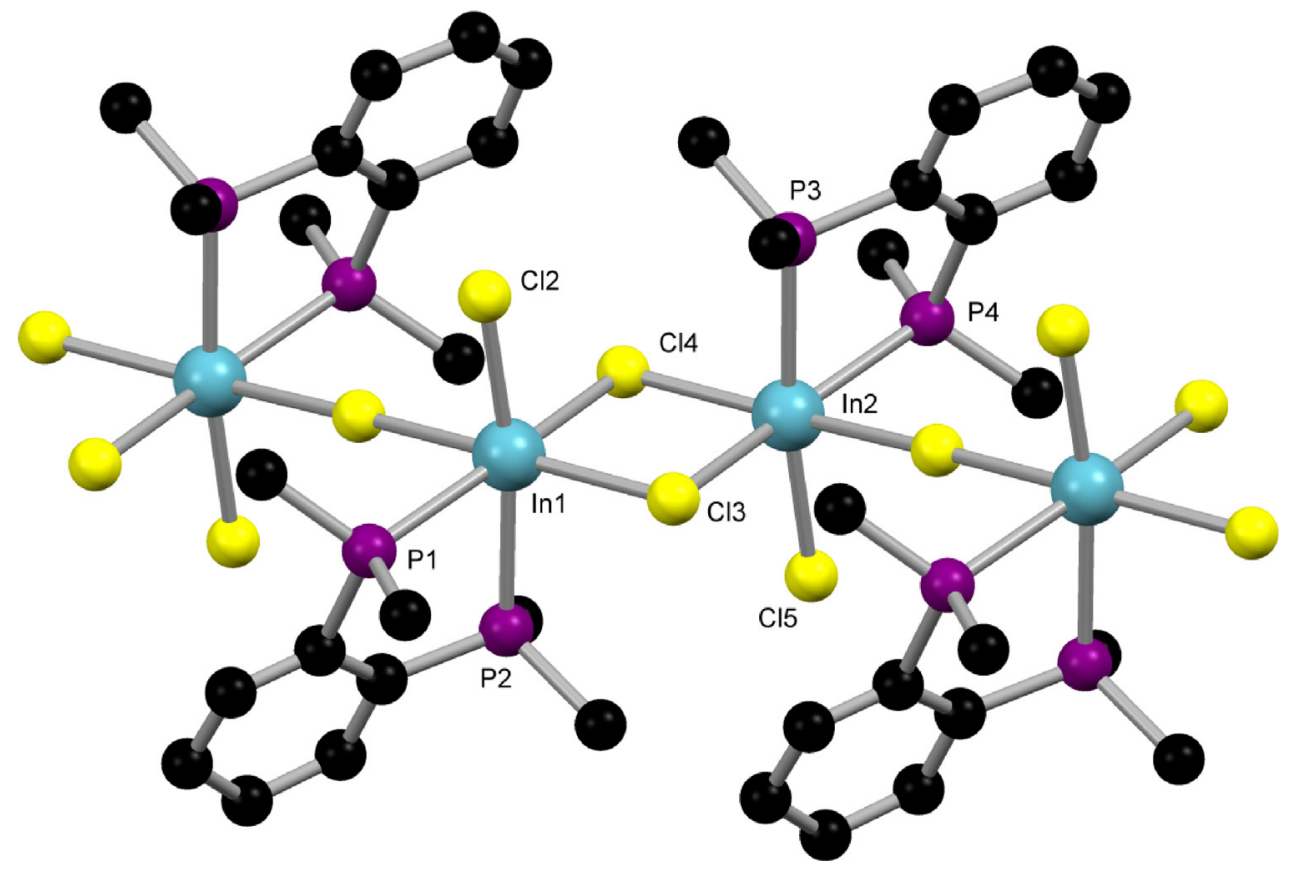

Fig. 39. View of the chain structure of the cation $\left[\mathrm{In}_{2} \mathrm{Cl}_{5}\left\{0-\mathrm{C}_{6} \mathrm{H}_{4}\left(\mathrm{PMe}_{2}\right)_{2}\right\}_{2}\right]^{+}$redrawn from Ref. [232].

bipyramidal $\left(\mathrm{C}_{3} \mathrm{P}_{2}\right)$, in contrast to the tetrahedral gallium complex (above) [162]. A chelating 3-(di-t-butylphosphino)propyl group is present in $\left[\mathrm{Me}_{2} \mathrm{In}\left\{\left(\mathrm{CH}_{2}\right)_{3} \mathrm{P}^{t} \mathrm{Bu}_{2}\right\}\right]$ [213], but $-\mathrm{CH}_{2} \mathrm{PPh}_{2}$ groups in $\left[\left\{\mathrm{R}_{2} \mathrm{In}\left(\mathrm{CH}_{2} \mathrm{PPh}_{2}\right)\right\}_{2}\right] \quad\left(\mathrm{R}=\mathrm{CH}_{2} \mathrm{CMe}_{3}, \mathrm{CH}_{2} \mathrm{SiMe}_{3}\right)$ bridge, giving dimeric species with six-membered rings In-C-P-In-C-P in a chair conformation [214]. Chelation is present in $\left[\mathrm{InY}_{2}\left\{\mathrm{C}_{5} \mathrm{H}_{4}\left(\mathrm{CH}_{2}\right)_{2} \mathrm{P}^{\mathrm{t}} \mathrm{Bu}_{2}\right\}\right](\mathrm{Y}=\mathrm{Me}$ or $\mathrm{Cl})$, which have $\eta^{1}$-coordination of the cyclopentadienyl ring, and hence distorted tetrahedral indium [215]. Chelating C,P-coordination is also found in the closo-2-[(dimethylphosphino)methyl]-1,2-dicarbaborane (HCB) complex [ $\left.\operatorname{InCl}(\mathbf{C B})_{2}\right]$ [165].

Indium(III) complexes with various anionic chelating ligands incorporating alkoxide, thiolate or amido donor groups in addition to neutral Group 15 donor ligands are generally similar to the gallium analogues. These include $\mathrm{Ph}_{2} \mathrm{ECH}_{2} \mathrm{CHRO}^{-}$and $\mathrm{Ph}_{2} \mathrm{ECH}_{2} \mathrm{CHRS}^{-}$ $(\mathrm{E}=\mathrm{P}, \mathrm{Sb}$ or $\mathrm{Bi})$, which form adducts with $\operatorname{In}^{i} \mathrm{Pr}_{2}$ groups [216], and $\mathrm{PPh}_{2}\left(\mathrm{o}-\mathrm{C}_{6} \mathrm{H}_{3} \mathrm{R}(\mathrm{OH})\right)$ and $\mathrm{PPh}\left(\mathrm{o}-\mathrm{C}_{6} \mathrm{H}_{3} \mathrm{R}(\mathrm{OH})\right)_{2}$ which coordinate in various modes via the phosphino groups and phenolate anions [242]. Adducts of tertiary phosphines with indium(III) thiolates are formed from $\left[\operatorname{In}\left(p-\mathrm{R}^{\prime} \mathrm{C}_{6} \mathrm{H}_{4} \mathrm{~S}\right)_{3}\right]$ and the phosphine, and are of type $\left[\operatorname{In}\left(p-\mathrm{R}^{\prime} \mathrm{C}_{6} \mathrm{H}_{4} \mathrm{~S}\right)_{3}\left(\mathrm{PR}_{3}\right)\right]\left(\mathrm{R}^{\prime}=\mathrm{H}, \mathrm{Me}, \mathrm{F} ; \mathrm{R}=\mathrm{Cy}, \mathrm{Et}, \mathrm{Ph}\right)$, being monomeric (distorted tetrahedral) in both solution and the solid state [243]. Similar complexes made from [ $\left.\mathrm{InH}_{3}\left(\mathrm{PCy}_{3}\right)\right]$ and $\mathrm{R}_{2} \mathrm{~S}_{2}$ were described above. Electrochemical dissolution of an indium anode in MeCN solutions of phosphinothiols including $o-\mathrm{Ph}_{2} \mathrm{PC}_{6} \mathrm{H}_{4} \mathrm{SH}$ and 2- $\mathrm{Ph}_{2} \mathrm{P}-6-\mathrm{Me}_{3} \mathrm{Si}_{-} \mathrm{C}_{6} \mathrm{H}_{3} \mathrm{SH}$, gave six-coordinate $\left(\mathrm{P}_{3} \mathrm{~S}_{3}\right.$-coordinated) complexes containing the corresponding phosphinothiolate [244]. The amino-diphosphine pincer (III-H) (see Section 6.3) bonds as a tridentate monoanion ( $\mathrm{NP}_{2}$-coordination) in trigonal bipyramidal [ $\mathrm{InX}_{2}$ (III)] $(\mathrm{X}=\mathrm{Cl}$ or $\mathrm{Br})$ [217]. Reduction of the macrocyclic diamidodiphosphine complex (structure of $\left(\mathbf{P}_{\mathbf{2}} \mathbf{N}_{\mathbf{2}}\right.$ ) given in Section 6.2) syn- $\left[\mathrm{InCl}\left(\mathbf{P}_{\mathbf{2}} \mathbf{N}_{\mathbf{2}}\right)\right]$ with $\mathrm{KC}_{8}$ produces the dimer $\left[\left\{\operatorname{In}\left(\mathbf{P}_{\mathbf{2}} \mathbf{N}_{\mathbf{2}}\right)\right\}_{2}\right]$, which has an In-In bond [169].

\subsection{Thallium}

In contrast to the lighter elements, thallium phosphines are very rare and no thallium examples with arsines or stibines are known. The only structurally authenticated neutral ligand example is $\left[\left(\mathrm{Me}_{3} \mathrm{SiCH}_{2}\right)_{3} \mathrm{Tl}\left\{\mathrm{P}\left(\mathrm{SiMe}_{3}\right)_{3}\right\}\right]$ (Fig. 40) obtained by direct combination of the silylphosphine and $\left[\left(\mathrm{Me}_{3} \mathrm{SiCH}_{2}\right)_{3} \mathrm{Tl}\right]$. The structure contains a distorted tetrahedral Tl(III) centre with Tl-P=1.922(3) $\AA$ [245]. All other examples contain phosphino groups in anionic ligands, which, due to the scarcity of Tl-P bonds, are briefly described here. The reaction of $\mathrm{TlCl}$ with $\mathrm{Li}\left[0-\mathrm{Ph}_{2} \mathrm{PCH}_{2} \mathrm{C}_{6} \mathrm{H}_{4}\right]$ results in disproportionation and formation of the $\mathrm{Tl}(\mathrm{III})$ complex $\left[\mathrm{Tl}\left(o-\mathrm{Ph}_{2} \mathrm{PCH}_{2} \mathrm{C}_{6} \mathrm{H}_{4}\right)_{3}\right]$ which has a tetrahedral thallium centre coordinated to three arene-C atoms, with a long bond to one of the

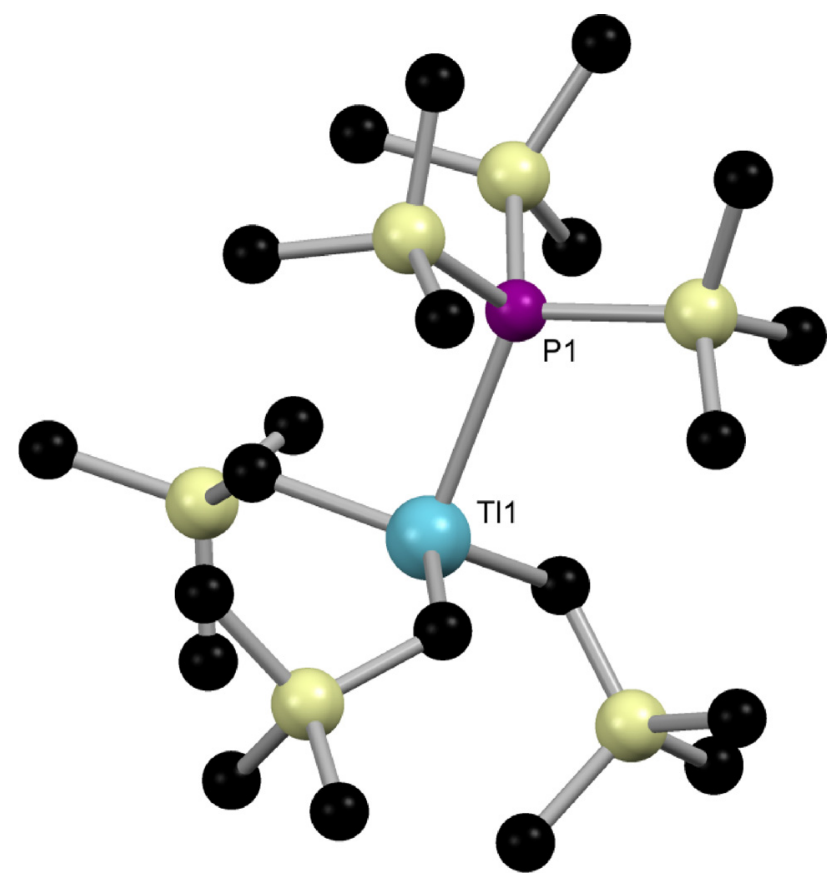

Fig. 40. View of the structure of $\left[\left(\mathrm{Me}_{3} \mathrm{SiCH}_{2}\right)_{3} \mathrm{Tl}\left\{\mathrm{P}\left(\mathrm{SiMe}_{3}\right)_{3}\right\}\right]$ redrawn from Ref. [245]. 
phosphine groups $(\mathrm{Tl}-\mathrm{P}=2.795(1) \AA)$ [162]. In contrast, the anionic PNP ligand (I) (structure in Section 6.2) forms the T-shaped $\mathrm{Tl}(\mathrm{I})$ complex on reaction with thallium(I) triflate [246]. The complex is unstable in air and has limited thermal stability, but is an effective ligand transfer reagent for the synthesis of some d-block metalPNP complexes. Peters and co-workers have prepared several $\mathrm{Tl}(\mathrm{I})$ complexes with phosphinoborates [247-252]. The diphosphinoborates include $\left[\mathrm{Tl}\left\{\mathrm{Ph}_{2} \mathrm{~B}\left(\mathrm{CH}_{2} \mathrm{PPh}_{2}\right)_{2}\right\}\right]$ (chain polymer with thallium coordinated $\eta^{6}$ to one $\mathrm{Ph}$ ring and one $-\mathrm{PPh}_{2}$ from each of two $\left[\mathrm{Ph}_{2} \mathrm{~B}\left(\mathrm{CH}_{2} \mathrm{PPh}_{2}\right)_{2}\right]^{-}$ligands $)$and $\left.\left[\mathrm{Tl}\left\{3-\mathrm{MeC}_{6} \mathrm{H}_{4}\right)_{2} \mathrm{~B}\left(\mathrm{CH}_{2} \mathrm{P}^{t} \mathrm{Bu}_{2}\right)_{2}\right\}\right]$ in which the ligand chelates via the two phosphine groups and forms an interaction best described as $\eta^{2}$ with the arene ring of another ligand. Triphosphinoborates, $\mathrm{PhB}\left(\mathrm{CH}_{2} \mathrm{P}^{\mathrm{i}} \mathrm{Pr}_{2}\right)_{3}$ and $\mathrm{PhB}\left(\mathrm{CH}_{2} \mathrm{PPh}_{2}\right)_{3}$, coordinate $\mathrm{K}^{3}$ to $\mathrm{Tl}(\mathrm{I})$, but whereas the former is monomeric, the latter exhibits weak Tl-Tl contacts in the solid state. The thallium salts, which are usually made by transmetallation from the lithium phosphinoborates, are useful reagents for the synthesis of transition metal complexes [252].

\section{Group 14}

Complexes of Sn(IV) with phosphine and arsine ligands are well known, and recent work has greatly extended knowledge of both $\mathrm{Ge}(\mathrm{IV})$ and $\mathrm{Ge}(\mathrm{II})$ complexes. Some tin(II) complexes have also been made. Silicon(IV) phosphines, although first reported in the 1960s, have been neglected until recently, and there are very little data indeed on lead compounds.

DFT calculations have explored the Lewis acid-Lewis base bonding in Group 14 elements, with the emphasis on silicon complexes with nitrogen donors, and comparisons with germanium analogues [253-256]. More recently $N$-heterocyclic carbene adducts have been similarly modelled $[257,258]$. DFT calculations on the latter have suggested that the carbenes will give more stable complexes than amines or phosphines with silicon or germanium, but limited specific effort has been devoted to phosphines, and even less to the heavier Group 15 donor ligands. However, the results on the $\mathrm{Si}-\mathrm{N}$ or $\mathrm{Si}-\mathrm{C}$ systems explored the major energy terms (these are gas phase calculations as usual, so solvation or lattice effects are not included): (a) the deformation energy needed to convert the tetrahedral $\mathrm{MX}_{4}$ unit to the four-coordinate fragment of either a trigonal bipyramid or an octahedron, which is endothermic and decreases $\mathrm{Si}>\mathrm{Ge}$ and with halide $\mathrm{F}>\mathrm{Cl}>\mathrm{Br}$, and $(\mathrm{b})$ the exothermic Lewis acid-Lewis base bond energy, which decreases with halide $\mathrm{F}>\mathrm{Cl}>\mathrm{Br}$ and from $\mathrm{Si}>\mathrm{Ge}$. The balance of these energies is that the germanium complexes are predicted to be more stable, since although the bond energies are lower than for analogous complexes of silicon, the deformation energy fall from $\mathrm{Si}$ to Ge is larger, so that overall the energy terms favour germanium. The poor Lewis acidity of silanes such as $\mathrm{SiH}_{4}$ or $\mathrm{SiH}_{3} \mathrm{Cl}$ is largely attributed to high deformation energies. One should note that the two large energy terms have opposing signs and in some cases nearly cancel out, so that smaller energy terms like intermolecular packing in the solid state may become significant. In the absence of detailed calculations, one can only extrapolate these results to heavier Group 15 donors, predicting that with germanium and especially silicon, the poorer match with the donor orbital size and energy will result in lower bond energies, less able to compensate for the large deformation energies, and this orbital mis-match will increase with As or Sb. Calculations suggest that $\mathrm{Ge}(\mathrm{II}), \mathrm{Sn}(\mathrm{II})$ and $\mathrm{Pb}(\mathrm{II})$ phosphines are viable complexes [258], in line with experimental data.

\subsection{Silicon}

Studies in the 1960 s reported $\left[\mathrm{SiX}_{4}\left(\mathrm{PMe}_{3}\right)_{2}\right](\mathrm{X}=\mathrm{Cl}$ or $\mathrm{Br})$, believed to be trans isomers from their vibrational spectra and from a low precision X-ray determination of the geometry of the chloride complex. The possible formation of $\mathrm{SiF}_{4}$ adducts at low temperatures was also reported [7]. Recent DFT calculations indicated that five-coordinate silicon(IV) halide phosphine complexes $\left[\mathrm{SiX}_{4}\left(\mathrm{PMe}_{3}\right)\right]$ are unlikely to be stable entities, that six-coordinate $\left[\mathrm{SiX}_{4}\left(\mathrm{PMe}_{3}\right)_{2}\right]$ would be of borderline stability, but that silicon(II) complexes [ $\mathrm{SiX}_{2}\left(\mathrm{PR}_{3}\right)$ ] should be formed exothermically [258].

Recent attempts to isolate phosphine or diphosphine complexes of $\mathrm{SiF}_{4}$ have been unsuccessful, and ${ }^{19} \mathrm{~F}$ and ${ }^{31} \mathrm{P}\left\{{ }^{1} \mathrm{H}\right\} \mathrm{NMR}$ studies showed no evidence for complex formation between the constituents in $\mathrm{CH}_{2} \mathrm{Cl}_{2}$ solution down to $190 \mathrm{~K}$ [259]. Old reports [260] of $\left[\mathrm{SiF}_{4}\left(\mathrm{PMe}_{3}\right)_{n}\right]$ ( $n=1$ or 2 ) formed at low temperatures in the absence of solvent, and identified by their Raman spectra, are not necessarily contradicted by the NMR results, since the experimental conditions are different, and these would merit reinvestigation. Very recently the trans- $\left[\mathrm{SiX}_{4}\left(\mathrm{PMe}_{3}\right)_{2}\right](\mathrm{X}=\mathrm{Cl}$ or $\mathrm{Br})$ have been re-examined [261]. They are readily isolated by combination of the constituents in $\mathrm{CH}_{2} \mathrm{Cl}_{2}$, and although very sensitive to water, are otherwise stable complexes. The structures show both to be trans isomers with little difference in the Si-P distances ( $\mathrm{X}=\mathrm{Cl}: 2.3483(3) \AA, \mathrm{X}=\mathrm{Br}: 2.359(2) \AA)$ and the NMR data show no significant dissociation in solution in $\mathrm{CH}_{2} \mathrm{Cl}_{2}$ at ambient temperatures. Attempts to prepare complexes with $\mathrm{PPh}_{3}$ were unsuccessful, probably due to the weaker donor power, and significantly, no reaction was observed with bulky but very strong $\sigma$-donor phosphines like $\mathrm{PBz}_{3}, \mathrm{PCy}_{3}$ or $\mathrm{P}^{t} \mathrm{Bu}_{3}$; the latter cases are consistent with the instability predicted for five-coordinate [SiX $\left.{ }_{4}\left(\mathrm{PR}_{3}\right)\right]$ [258], and these phosphines are too large to achieve six-coordination on the small silicon centre. No reaction occurred with $\mathrm{AsMe}_{3}$, which is only slightly larger than $\mathrm{PMe}_{3}$, suggesting that here the (assumed) weaker $\mathrm{Si}-\mathrm{As}$ bond does not compensate for the deformation energy [261]. A range of diphosphine complexes cis-[SiX $(\mathrm{L}-\mathrm{L})]\left(\mathrm{X}=\mathrm{Cl}\right.$ or $\mathrm{Br}$; $\mathrm{L}-\mathrm{L}=\mathrm{Me}_{2} \mathrm{P}\left(\mathrm{CH}_{2}\right)_{2} \mathrm{PMe}_{2}$, $\mathrm{Et}_{2} \mathrm{P}\left(\mathrm{CH}_{2}\right)_{2} \mathrm{PEt}_{2}$ or $\left.\mathrm{o}-\mathrm{C}_{6} \mathrm{H}_{4}\left(\mathrm{PMe}_{2}\right)_{2}\right)$ was isolated and all characterised by $\mathrm{X}$-ray structure determinations and multinuclear NMR spectroscopy, the latter showing no evidence for dissociation of the phosphines in solution [261]. The diphosphine $\mathrm{Me}_{2} \mathrm{PCH}_{2} \mathrm{PMe}_{2}$ gave trans-[ $\left.\mathrm{SiCl}_{4}\left(\kappa^{1}-\mathrm{Me}_{2} \mathrm{PCH}_{2} \mathrm{PMe}_{2}\right)_{2}\right]$ (Fig. 41), but no complexation occurred with $0-\mathrm{C}_{6} \mathrm{H}_{4}\left(\mathrm{AsMe}_{2}\right)_{2}$. The reaction of $\mathrm{SiHCl}_{3}$ with $\mathrm{PMe}_{3}, \mathrm{Me}_{2} \mathrm{P}\left(\mathrm{CH}_{2}\right)_{2} \mathrm{PMe}_{2}$ or $0-\mathrm{C}_{6} \mathrm{H}_{4}\left(\mathrm{PMe}_{2}\right)_{2}$ in the solution gave the $\mathrm{SiCl}_{4}$ complexes described above, resulting from disproportionation of the silane. However, with $\mathrm{Et}_{2} \mathrm{P}\left(\mathrm{CH}_{2}\right)_{2} \mathrm{PEt}_{2}$ the product was crystalline cis-[ $\left.\mathrm{SiHCl}_{3}\left(\mathrm{Et}_{2} \mathrm{P}\left(\mathrm{CH}_{2}\right)_{2} \mathrm{PEt}_{2}\right)\right]$ (Fig. 42). In solution at room temperature the diphosphine complex is extensively dissociated, showing the weaker Lewis acidity of the $\mathrm{SiHCl}_{3}$, but low temperature NMR studies were fully consistent with the solid state structure [261]. The reactions of $\mathrm{Si}_{2} \mathrm{Cl}_{6}$ with phosphines in $\mathrm{CH}_{2} \mathrm{Cl}_{2}$ solution resulted in formation of the corresponding $\mathrm{SiCl}_{4}$ complexes and yellow oligochlorosilanes, but reactions in hexane produced white powders which were identified as $\left[\mathrm{Cl}_{3} \mathrm{SiSiCl}_{3}\left(\mathrm{PMe}_{3}\right)_{2}\right]$ or $\left[\mathrm{Cl}_{3} \mathrm{SiSiCl}_{3}\left(\mathrm{Me}_{2} \mathrm{P}\left(\mathrm{CH}_{2}\right)_{2} \mathrm{PMe}_{2}\right)\right]$ by microanalysis and IR spectroscopy. These decompose immediately in chlorocarbon solvents, and more slowly in hexane, and therefore no solution measurements were possible [261]. The diamine analogue $\left[\mathrm{Cl}_{3} \mathrm{SiSiCl}_{3}\left(\mathrm{Me}_{2} \mathrm{~N}\left(\mathrm{CH}_{2}\right)_{2} \mathrm{NMe}_{2}\right)\right]$ is more stable and the X-ray structure has been determined [262]. Attempts to reduce silicon(IV) phosphines to $\mathrm{Si}(\mathrm{II})$ have so far failed [261].

\subsection{Germanium}

Work reported pre-1975 described a series of $\left[\mathrm{GeI}_{2}\left(\mathrm{PR}_{3}\right)\right]$ complexes and two examples with $\mathrm{GeCl}_{4}$, viz $\left[\mathrm{GeCl}_{4}\left(\mathrm{PMe}_{3}\right)_{n}\right](n=1$ or 2) [7].

In marked contrast to the failure of $\mathrm{SiF}_{4}$ to form phosphine complexes, gaseous $\mathrm{GeF}_{4}$, or more conveniently, $\left[\mathrm{GeF}_{4}(\mathrm{MeCN})_{2}\right]$ [263], react readily with $\mathrm{PPh}_{3}$ or $\mathrm{PMe}_{3}$ to form white solids, 


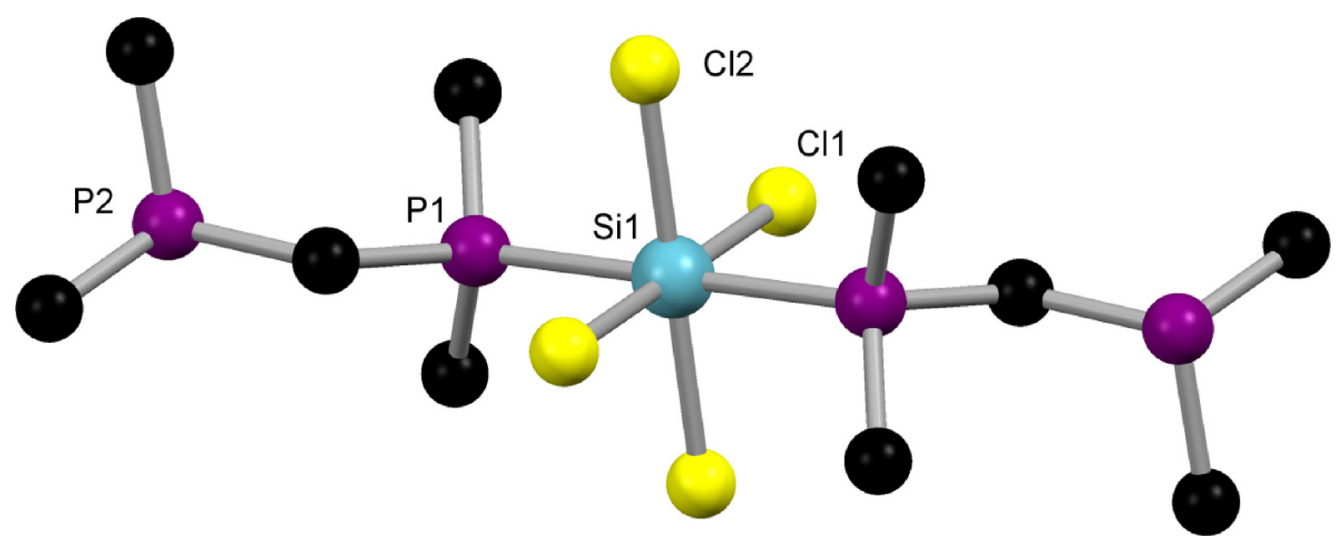

Fig. 41. View of the structure of $\left[\mathrm{SiCl}_{4}\left(\kappa^{1}-\mathrm{Me}_{2} \mathrm{PCH}_{2} \mathrm{PMe}_{2}\right)_{2}\right]$ redrawn from Ref. [261].

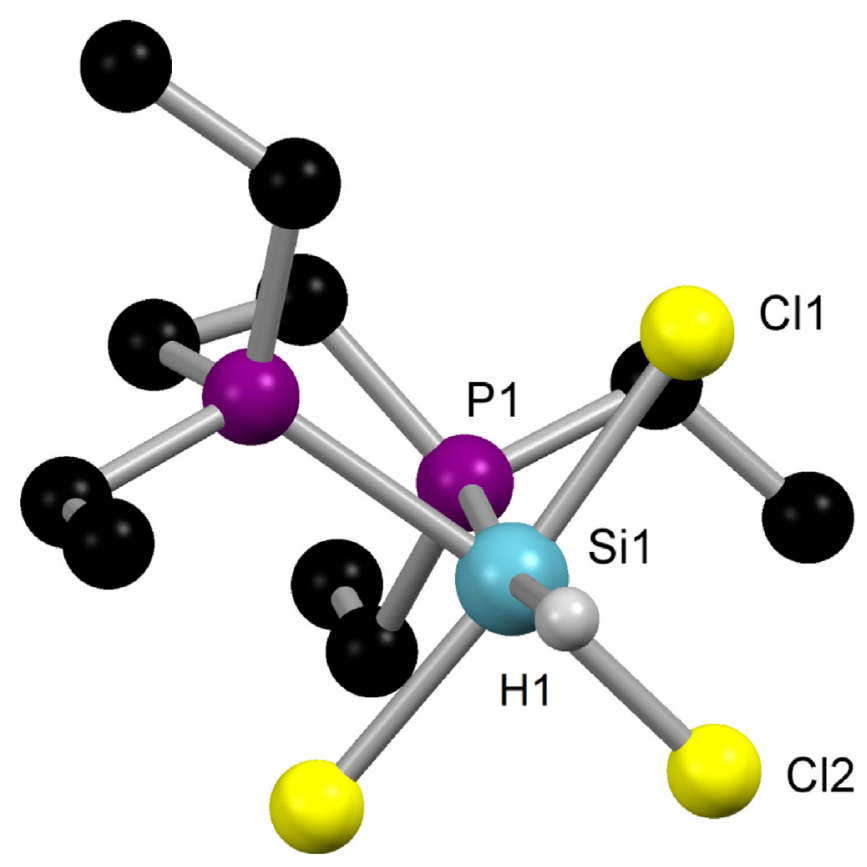

Fig. 42. View of the structure of $\left[\mathrm{SiHCl}_{3}\left\{\mathrm{Et}_{2} \mathrm{P}\left(\mathrm{CH}_{2}\right)_{2} \mathrm{PEt}_{2}\right\}\right]$ redrawn from Ref. [261]. trans- $\left[\mathrm{GeF}_{4}\left(\mathrm{PR}_{3}\right)_{2}\right]$, whilst the diphosphines $\mathrm{R}_{2} \mathrm{P}\left(\mathrm{CH}_{2}\right)_{2} \mathrm{PR}_{2}(\mathrm{R}=\mathrm{Me}$, Et, $\mathrm{Cy}$ or $\mathrm{Ph})$ and $\mathrm{o}-\mathrm{C}_{6} \mathrm{H}_{4}\left(\mathrm{PR}_{2}\right)_{2}(\mathrm{R}=\mathrm{Me}$ or $\mathrm{Ph})$ afford cis[ $\mathrm{GeF}_{4}$ (diphosphine)] [264]. Multinuclear NMR studies show that trans- $\left[\mathrm{GeF}_{4}\left(\mathrm{PPh}_{3}\right)_{2}\right]$ is extensively dissociated in $\mathrm{CH}_{2} \mathrm{Cl}_{2}$ solution, but the other complexes are stable in solution, although sensitive to both moisture and oxygen. The complexes of air-stable free phosphines $\left(\mathrm{PPh}_{3}\right.$ and $\left.\mathrm{Ph}_{2} \mathrm{P}\left(\mathrm{CH}_{2}\right)_{2} \mathrm{PPh}_{2}\right)$ are slowly converted to the corresponding phosphine oxide complexes when their $\mathrm{GeF}_{4}$ adducts are exposed to dry dioxygen in $\mathrm{CH}_{2} \mathrm{Cl}_{2}$ solution; use of ${ }^{18} \mathrm{O}_{2}$ confirmed dioxygen rather than hydrolysis is involved [264]. $\mathrm{X}$-Ray crystal structures of two examples $\left[\mathrm{GeF}_{4}\left\{\mathrm{Ph}_{2} \mathrm{P}\left(\mathrm{CH}_{2}\right)_{2} \mathrm{PPh}_{2}\right\}\right]$ (Fig. 43) and [ $\left.\mathrm{GeF}_{4}\left\{0-\mathrm{C}_{6} \mathrm{H}_{4}\left(\mathrm{PMe}_{2}\right)_{2}\right\}\right]$ (Table 10) were determined, and detailed NMR and vibrational spectroscopic data reported. The coordination shifts in the ${ }^{31} \mathrm{P}\left\{{ }^{1} \mathrm{H}\right\}$ NMR spectra are again erratic, with both high and low frequency shifts being observed in different systems. Attempts to characterise arsine complexes of $\mathrm{GeF}_{4}$ were unsuccessful; although white solids were isolated with $\mathrm{AsMe}_{3}$ or $o-\mathrm{C}_{6} \mathrm{H}_{4}\left(\mathrm{AsMe}_{2}\right)_{2}$, these were very unstable and not identified.

The reactions of $\mathrm{GeCl}_{4}$ and $\mathrm{GeBr}_{4}$ with phosphine ligands proved to be very sensitive to the reaction conditions, and a clear picture of the chemistry has emerged only recently. The reaction of $\mathrm{GeCl}_{4}$ with $\mathrm{PMe}_{3}$ in the absence of a solvent was reported [265] to give trans-[ $\left.\mathrm{GeCl}_{4}\left(\mathrm{PMe}_{3}\right)_{2}\right]$ identified by vibrational spectroscopy, which on reaction with excess $\mathrm{GeCl}_{4}$ gave five-coordinate

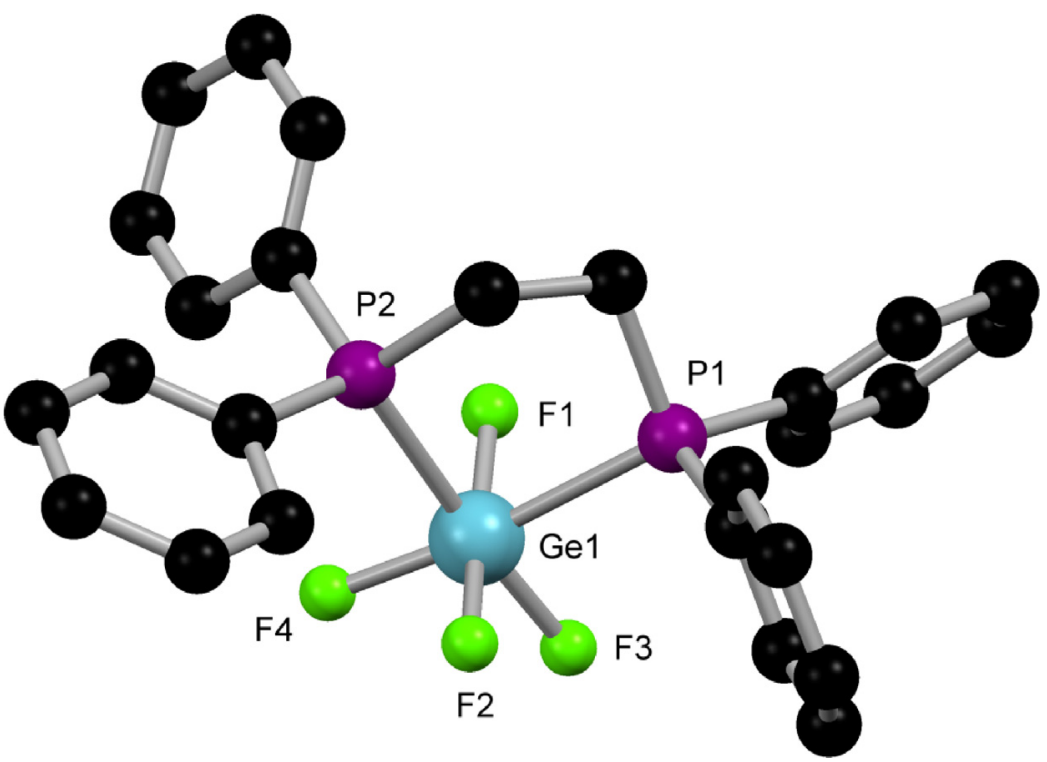

Fig. 43. View of the structure of $\left[\mathrm{GeF}_{4}\left\{\mathrm{Ph}_{2} \mathrm{P}\left(\mathrm{CH}_{2}\right)_{2} \mathrm{PPh}_{2}\right\}\right]$ redrawn from Ref. [264]. 
Table 10

$\mathrm{X}$-ray structures of germanium complexes.

\begin{tabular}{|c|c|c|c|c|}
\hline Complex & Ge-P/As $(\AA)$ & $\mathrm{Ge}-\mathrm{X}(\AA)$ & Comments & Ref. \\
\hline$\left[\mathrm{GeF}_{4}\left\{\mathrm{Ph}_{2} \mathrm{P}\left(\mathrm{CH}_{2}\right)_{2} \mathrm{PPh}_{2}\right\}\right]$ & $2.436(7), 2.4827(7)$ & $1.7692(14)-1.7987(14)$ & & [264] \\
\hline$\left[\mathrm{GeF}_{4}\left\{\mathrm{o}-\mathrm{C}_{6} \mathrm{H}_{4}\left(\mathrm{PMe}_{2}\right)_{2}\right\}\right]$ & $2.4273(12)$ & $1.765(2)-1.815(2)$ & & [264] \\
\hline trans-[$\left[\mathrm{GeCl}_{4}\left(\mathrm{AsMe}_{3}\right)_{2}\right]$ & $2.472(1)$ & $2.307(4)-2.341(4)$ & & [270] \\
\hline trans- $\left[\mathrm{GeCl}_{4}\left(\mathrm{AsEt}_{3}\right)_{2}\right]$ & $2.4904(9)$ & $2.3296(19), 2.3233(19)$ & & [264] \\
\hline$\left[\mathrm{GeI}_{2}\left(\mathrm{PPh}_{3}\right)\right]$ & $2.503(4), 2.510(5)$ & $2.634(2)-2.661(3)$ & Pyramidal & [273] \\
\hline$\left[\mathrm{GeCl}_{2}\left(\mathrm{PPh}_{3}\right)\right]$ & $2.5084(7)$ & $2.2598(2), 2.2687(8)$ & Pyramidal & {$[274]$} \\
\hline$\left[\mathrm{GeCl}_{2}\left\{\mathrm{Ph}_{2} \mathrm{P}\left(\mathrm{CH}_{2}\right)_{2} \mathrm{PPh}_{2}\right\}\right]$ & $2.514(2)$ & $2.236(2), 2.332(2)$ & $\kappa^{1}$-Diphosphine & [276] \\
\hline$\left[\mathrm{GeCl}_{2}\left\{0-\mathrm{C}_{6} \mathrm{H}_{4}\left(\mathrm{PPh}_{2}\right)_{2}\right\}\right]$ & $2.5153(13), 3.1958(15)$ & $2.2753(15), 2.3195(16)$ & Approx $\kappa^{1}$-diphosphine & [277] \\
\hline$\left[\mathrm{GeBr}_{2}\left\{0-\mathrm{C}_{6} \mathrm{H}_{4}\left(\mathrm{PPh}_{2}\right)_{2}\right\}\right]$ & $2.5259(13), 3.1167(14)$ & $2.4635(7), 2.5057(8)$ & Approx $\kappa^{1}$-diphosphine & [277] \\
\hline$\left[\mathrm{GeI}_{2}\left\{\mathrm{o}-\mathrm{C}_{6} \mathrm{H}_{4}\left(\mathrm{PPh}_{2}\right)_{2}\right\}\right]$ & $2.511(2), 3.068(2)$ & $2.6733(11), 2.7663(11)$ & Approx $\kappa^{1}$-diphosphine & [277] \\
\hline$\left[\mathrm{GeCl}_{2}\left\{0-\mathrm{C}_{6} \mathrm{H}_{4}\left(\mathrm{PMe}_{2}\right)_{2}\right\}\right]$ & $2.4709(11), 2.4390(11)$ & $2.4942(11), 2.7047(11)$ & Dimer & [277] \\
\hline$\left[\mathrm{GeBr}_{2}\left\{0-\mathrm{C}_{6} \mathrm{H}_{4}\left(\mathrm{PMe}_{2}\right)_{2}\right\}\right]$ & $2.4805(9), 2.4498(10)$ & $2.6251(6), 2.8259(6)$ & Dimer & [277] \\
\hline$\left[\mathrm{GeI}_{2}\left\{\mathrm{o}-\mathrm{C}_{6} \mathrm{H}_{4}\left(\mathrm{PMe}_{2}\right)_{2}\right\}\right]$ & $2.481(2), 2.456(2)$ & $2.8211(12), 3.0749(12)$ & Dimer & [277] \\
\hline$\left[\mathrm{GeCl}_{2}\left\{\mathrm{Me}_{2} \mathrm{P}\left(\mathrm{CH}_{2}\right)_{2} \mathrm{PMe}_{2}\right\}\right]$ & $2.4495(6)$ & $2.5858(7)$ & Monomer & [277] \\
\hline$\left[\mathrm{GeBr}_{2}\left\{\mathrm{Me}_{2} \mathrm{P}\left(\mathrm{CH}_{2}\right)_{2} \mathrm{PMe}_{2}\right\}\right]$ & $2.4628(7)$ & $2.7335(5)$ & Monomer & [277] \\
\hline$\left[\mathrm{GeI}_{2}\left\{\mathrm{Me}_{2} \mathrm{P}\left(\mathrm{CH}_{2}\right)_{2} \mathrm{PMe}_{2}\right\}\right]$ & $2.463(2), 2.514(2)$ & $2.8685(12), 3.0787(13)$ & Weak dimer & [277] \\
\hline$\left[\mathrm{GeCl}_{2}\left\{\mathrm{Et}_{2} \mathrm{P}\left(\mathrm{CH}_{2}\right)_{2} \mathrm{PEt}_{2}\right\}\right]$ & $2.4672(5)$ & $2.5803(5)$ & Monomer & [277] \\
\hline$\left[\mathrm{GeBr}_{2}\left\{\mathrm{Et}_{2} \mathrm{P}\left(\mathrm{CH}_{2}\right)_{2} \mathrm{PEt}_{2}\right\}\right]$ & $2.4694(5)$ & $2.7340(4)$ & Monomer & [277] \\
\hline$\left[\mathrm{GeI}_{2}\left\{0-\mathrm{C}_{6} \mathrm{H}_{4}\left(\mathrm{AsMe}_{2}\right)_{2}\right\}\right]$ & $2.6210(11), 2.613(11)$ & $2.8763(10), 3.0880(10)$ & Chain polymer & [277] \\
\hline$\left[\mathrm{GeCl}\left\{0-\mathrm{C}_{6} \mathrm{H}_{4}\left(\mathrm{AsMe}_{2}\right)_{2}\right\}\right]\left[\mathrm{GeCl}_{3}\right]$ & $2.5847(5)$ & $2.3734(13)$ & & {$[277]$} \\
\hline$\left[\mathrm{Ge}\left\{{ }^{t} \mathrm{Bu}_{2} \mathrm{PCH}_{2} \mathrm{C}\left(\mathrm{CF}_{3}\right)_{2} \mathrm{O}\right\}_{2}\right]$ & $2.7331(12), 2.7585(12)$ & & & [279] \\
\hline
\end{tabular}

$\left[\mathrm{GeCl}_{4}\left(\mathrm{PMe}_{3}\right)\right][266]$. However, reaction of $\mathrm{P}^{t} \mathrm{Bu}_{3}$ or $\mathrm{P}^{i} \mathrm{Pr}_{3}$ with $\mathrm{GeX}_{4}$ ( $\mathrm{X}=\mathrm{Cl}$ or $\mathrm{Br}$ ) [267-269] formed [ $\left.\mathrm{PR}_{3} \mathrm{X}\right]\left[\mathrm{GeX}_{3}\right]$ (which have the elemental composition "GeX $\left(\mathrm{PR}_{3}\right)$ "), and this was confirmed by a structure determination of $\left[\mathrm{P}^{i} \mathrm{Pr}_{3} \mathrm{Br}\right]\left[\mathrm{GeBr}_{3}\right]$. Subsequently Godfrey et al. [270] showed that the reaction of $\mathrm{GeCl}_{4}$ with a range of phosphines $\mathrm{PR}_{3}\left(\mathrm{R}=\mathrm{Me}, \mathrm{Et},{ }^{n} \mathrm{Bu}, \mathrm{Cy}, \mathrm{PhCH}_{2}, \mathrm{Me}_{2} \mathrm{~N}, 2,4,6-(\mathrm{MeO})_{3} \mathrm{C}_{6} \mathrm{H}_{2}\right)$ in diethyl ether solution gave $\left[\mathrm{PR}_{3} \mathrm{Cl}\right]\left[\mathrm{GeCl}_{3}\right]$, identified by microanalysis, comparison of the spectroscopic data with known $\left[\mathrm{PR}_{3} \mathrm{Cl}\right]^{+}$ salts and by a crystal structure of $\left[\mathrm{P}^{n} \mathrm{Bu}_{3} \mathrm{Cl}\right]\left[\mathrm{GeCl}_{3}\right]$. In some cases a second (minor) ${ }^{31} \mathrm{P}$ NMR resonance was observed, speculated as due to intermediate formation of a $\mathrm{Ge}(\mathrm{IV})$ complex. Re-examination of the $\mathrm{GeCl}_{4} / \mathrm{PMe}_{3}$ reaction [264] confirmed Beattie's original report [265] of the formation of trans-[ $\left[\mathrm{GeCl}_{4}\left(\mathrm{PMe}_{3}\right)_{2}\right]$ by co-condensation of $\mathrm{GeCl}_{4}$ with $\mathrm{PMe}_{3}$ at low temperatures, but showed that dissolution of the product in $\mathrm{CH}_{2} \mathrm{Cl}_{2}$ led to very rapid conversion into $\left[\mathrm{PMe}_{3} \mathrm{Cl}\right]\left[\mathrm{GeCl}_{3}\right]$. The reactions with $\mathrm{GeBr}_{4}$ appear to form $\left[\mathrm{PMe}_{3} \mathrm{Br}\right]\left[\mathrm{GeBr}_{3}\right]$ very rapidly and $\mathrm{Ge}(\mathrm{IV})$ intermediates are not detected. The reported $\left[\mathrm{GeBr}_{4}\left(\mathrm{PMe}_{3}\right)\right][266]$ has an identical IR spectrum to $\left[\mathrm{PMe}_{3} \mathrm{Br}\right]\left[\mathrm{GeBr}_{3}\right]$ and the latter is the correct formulation [264]. Diphosphines also react with $\mathrm{GeCl}_{4}$ to give $\left[\mathrm{GeCl}_{3}\right]^{-}$ salts [264]. Primary and secondary phosphines undergo similar, but more complicated chemistry with $\mathrm{GeCl}_{4}$ [271]. $\mathrm{PCyH}_{2}$ and $\mathrm{GeCl}_{4}$ in diethyl ether solution gave the crystallographically authenticated $\left[\mathrm{PCyH}_{3}\right]\left[\mathrm{GeCl}_{3}\right]$ and in situ spectroscopic studies identified $\left[\mathrm{GeCl}_{2}\left(\mathrm{PCyH}_{2}\right)\right]$ and $\left[\mathrm{GeCl}_{3}(\mathrm{PCyH})\right]$ as other products. Similarly $\mathrm{PPh}_{2} \mathrm{H}$ and $\mathrm{GeCl}_{4}$ gave $\left[\mathrm{PPh}_{2} \mathrm{H}_{2}\right]\left[\mathrm{GeCl}_{3}\right],\left[\mathrm{GeCl}_{2}\left(\mathrm{PPh}_{2} \mathrm{H}\right)\right]$ and $\mathrm{Ph}_{2} \mathrm{PCl}$ [271]. The germylphosphines such as $\left[\mathrm{GeCl}_{3}(\mathrm{PRH})\right]\left(\mathrm{R}=\mathrm{Ph},{ }^{t} \mathrm{Bu}\right.$, mesityl etc.) are produced quantitatively from $\mathrm{PRH}_{2}$ and excess $\mathrm{GeCl}_{4}[272]$.

Reactions of $\mathrm{AsMe}_{3}$ or $\mathrm{AsEt}_{3}$ with $\mathrm{GeCl}_{4}$ produced trans$\left[\mathrm{GeCl}_{4}\left(\mathrm{AsR}_{3}\right)_{2}\right]$, both confirmed by X-ray crystal structures $[264,270]$; in solution over several days these decompose forming $\mathrm{AsR}_{3} \mathrm{Cl}_{2}$ [264]. No reaction was found between $\mathrm{GeCl}_{4}$ and $o-\mathrm{C}_{6} \mathrm{H}_{4}\left(\mathrm{AsMe}_{2}\right)_{2}$ [264]. The successful isolation of $\left[\mathrm{GeCl}_{4}\left(\mathrm{AsR}_{3}\right)_{2}\right]$, in contrast to the transient formation of the corresponding phosphines, is readily attributed to the much greater reducing power of the phosphines. Comparison of the chemistry of $\mathrm{SiX}_{4}$ and $\mathrm{GeX}_{4}$ with tertiary phosphines and arsines reveals unexpectedly great differences. The failure to form $\mathrm{SiF}_{4}$ adducts compared to the stable $\left[\mathrm{GeF}_{4}\left(\mathrm{PR}_{3}\right)_{2}\right]$ is most probably traceable to the higher deformation energy of $\mathrm{SiF}_{4}$, but the formation of stable $\mathrm{SiCl}_{4}$ adducts, contrasted with the redox chemistry found with $\mathrm{GeCl}_{4}$, seems most likely to reflect the reduced stability of the group oxidation state in period 4 of the periodic table. This is clearly evident in the chemistry of

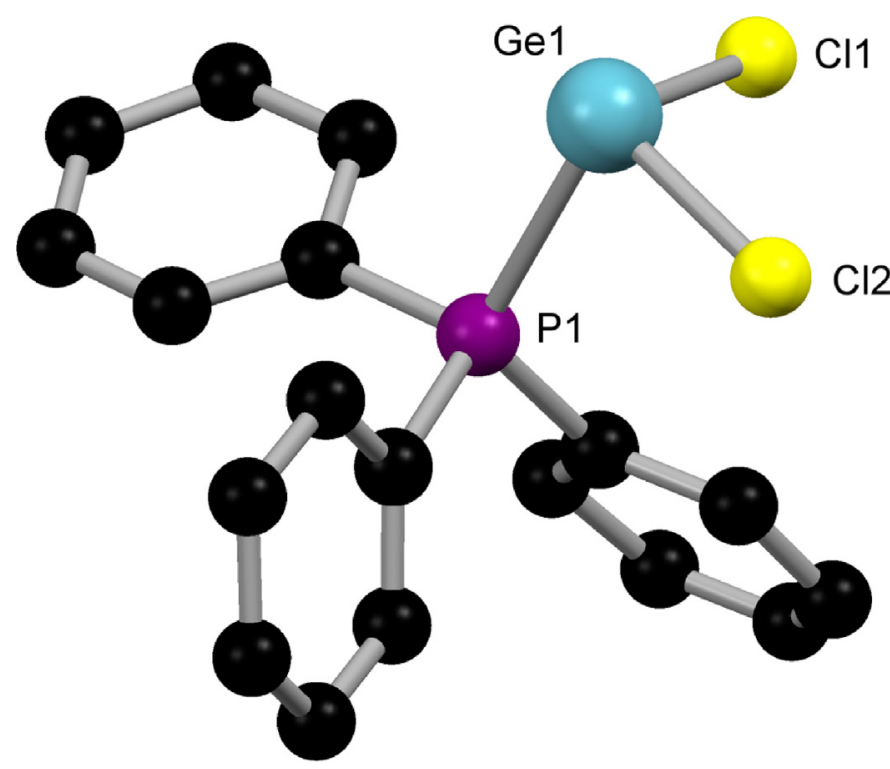

Fig. 44. View of the structure of $\left[\mathrm{GeCl}_{2}\left(\mathrm{PPh}_{3}\right)\right]$ redrawn from Ref. [274].

succeeding elements like $\mathrm{As}$, Se or $\mathrm{Br}$, and rationalised as a consequence of insertion of the first transition series on the ionisation potentials and orbital energies.

The first phosphine complexes of germanium(II), [GeX $\left.\left(\mathrm{PPh}_{3}\right)\right]$ $(\mathrm{X}=\mathrm{Cl}$ or $\mathrm{I})$ [273-275] were discrete pyramidal monomers with no short intermolecular contacts (Fig. 44), and recent DFT modeling of $\left[\mathrm{GeX}_{2}\left(\mathrm{PR}_{3}\right)\right]$ indicated these are stable complexes [258]. $\mathrm{GeCl}_{2}$ forms both $2: 1$ and $1: 1$ complexes with $\mathrm{Ph}_{2} \mathrm{P}\left(\mathrm{CH}_{2}\right)_{2} \mathrm{PPh}_{2}$; the former is probably $\left[\mathrm{Cl}_{2} \mathrm{Ge}\left\{\mu-\mathrm{Ph}_{2} \mathrm{P}\left(\mathrm{CH}_{2}\right)_{2} \mathrm{PPh}_{2}\right\} \mathrm{GeCl}_{2}\right]$, but the latter was shown by an X-ray structure determination to be $\left[\mathrm{GeCl}_{2}\left\{\mathrm{~K}^{1}-\right.\right.$ $\left.\left.\mathrm{Ph}_{2} \mathrm{P}\left(\mathrm{CH}_{2}\right)_{2} \mathrm{PPh}_{2}\right)\right]$ with a $\mathrm{Cl}_{2} \mathrm{P}$ core and the second phosphine group $\sim 3.3 \AA$ from the germanium [276].

The more rigid $o-\mathrm{C}_{6} \mathrm{H}_{4}\left(\mathrm{PPh}_{2}\right)_{2}$ forms similar $1: 1$ complexes with $\mathrm{GeX}_{2}$ (X = Cl, Br or I) (Fig. 45) [277] in which the Ge - P distances differ by $\sim 0.6 \AA$, with the second phosphine group interacting weakly $\left[3+1\right.$ coordination]. The ${ }^{31} \mathrm{P}$ NMR spectra of these three complexes show singlets with small high frequency coordination shifts even at low temperatures, which suggests that exchange between the two phosphine groups is fast in solution. In contrast, the very strong $\sigma$-donor $0-\mathrm{C}_{6} \mathrm{H}_{4}\left(\mathrm{PMe}_{2}\right)_{2}$ chelates to $\mathrm{GeX}_{2}(\mathrm{X}=\mathrm{Cl}, \mathrm{Br}$ or I) [277]; the 


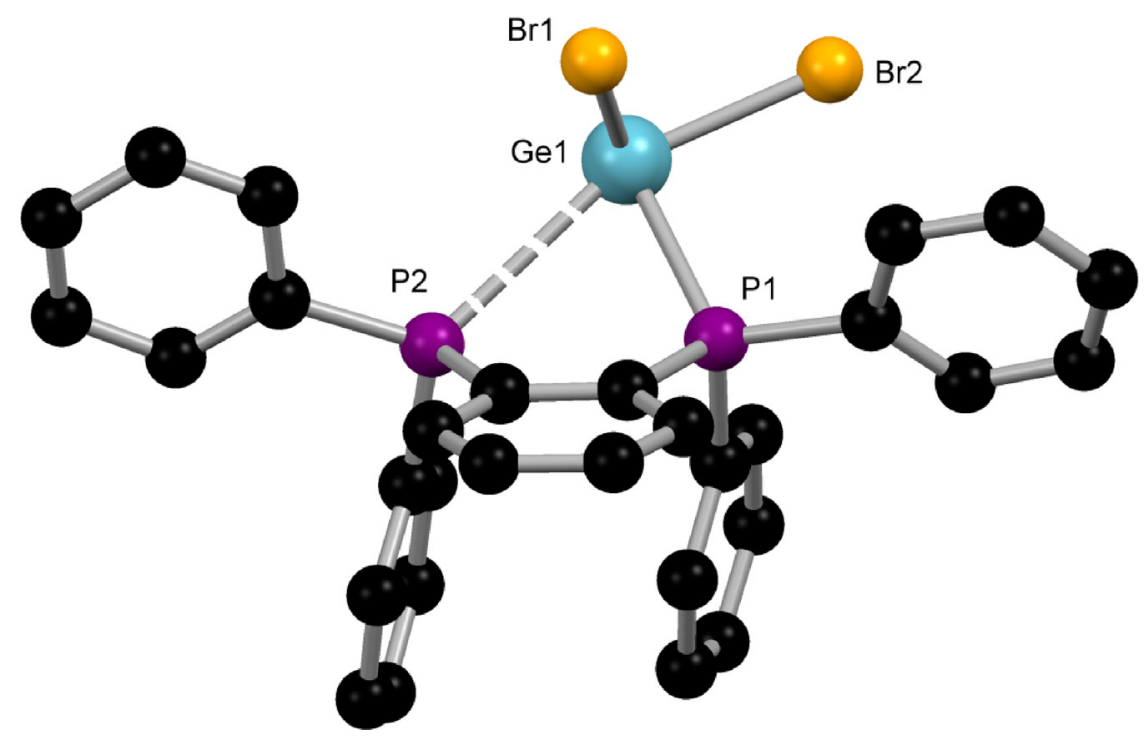

Fig. 45. View of the structure of $\left[\mathrm{GeBr}_{2}\left\{o-\mathrm{C}_{6} \mathrm{H}_{4}\left(\mathrm{PPh}_{2}\right)_{2}\right\}\right]$ redrawn from Ref. [277].

isomorphous chloride and bromide complexes are halide-bridged dimers (Fig. 46) with the germanium core of $\mathrm{P}_{2} \mathrm{X}_{2}$ and a long bond to a third $\mathrm{X}$, whereas the iodo-complex has a similar core, but with very long Ge.••I bridges, just within the van der Waals radii sum (Fig. 47).

The diphosphinoalkane complexes $\left[\mathrm{GeX}_{2}\left\{\mathrm{R}_{2} \mathrm{P}\left(\mathrm{CH}_{2}\right)_{2} \mathrm{PR}_{2}\right\}\right]$ ( $\mathrm{X}=\mathrm{Cl}$ or $\mathrm{Br}, \mathrm{R}=\mathrm{Me}$ or $\mathrm{Et}$ ) are different again, with discrete four-coordinate germanium centres, the structure approximating to a pseudo-trigonal bipyramid with a vacant equatorial vertex (Fig. 48) [277]. The core structure of $\left[\mathrm{GeI}_{2}\left\{\mathrm{Me}_{2} \mathrm{P}\left(\mathrm{CH}_{2}\right)_{2} \mathrm{PMe}_{2}\right\}\right]$ is similar but the geometry shows very long iodo-bridges forming dimer units. For all of these alkyl substituted diphosphine complexes, the ${ }^{31} \mathrm{P}\left\{{ }^{1} \mathrm{H}\right\}$ NMR spectra show singlets with large high frequency coordination shifts, which was interpreted as evidence for five-membered chelate rings present in solution [277].

The diarsine $0-\mathrm{C}_{6} \mathrm{H}_{4}\left(\mathrm{AsMe}_{2}\right)_{2}$ forms [ $\left.\mathrm{GeX}_{2}\left\{0-\mathrm{C}_{6} \mathrm{H}_{4}\left(\mathrm{AsMe}_{2}\right)_{2}\right\}\right]$ for $\mathrm{X}=\mathrm{Br}$ or $\mathrm{I}$, which have an $\mathrm{As}_{2} \mathrm{X}_{2}$ core similar to that in the alkyldiphosphine complexes, but are further weakly associated via halide bridges into a polymer chain [277]. Surprisingly
$\left[\mathrm{GeCl}_{2}\right.$ (dioxane)] and $0-\mathrm{C}_{6} \mathrm{H}_{4}\left(\mathrm{AsMe}_{2}\right)_{2}$ formed only [ $\mathrm{GeCl}\{0$ $\left.\left.\mathrm{C}_{6} \mathrm{H}_{4}\left(\mathrm{AsMe}_{2}\right)_{2}\right\}\right]\left[\mathrm{GeCl}_{3}\right]$ with pyramidal cations and anions forming a polymeric chain via longer chloride bridges [277]. The only examples of monodentate arsine complexes are $\left[\mathrm{GeCl}_{2}\left(\mathrm{AsR}_{3}\right)\right](\mathrm{R}=\mathrm{Ph}$ or ${ }^{t} \mathrm{Bu}$ ) which are probably pyramidal monomers, although the structures have not been determined [278].

The structural chemistry of $\mathrm{Ge}(\mathrm{II})$ phosphines is clearly complex. Whilst there is a preference for a pyramidal $\mathrm{PX}_{2}$ core with monodentate or weaker donor bidentates, strong chelating ligands produce four-coordinate $\mathrm{P}_{2} \mathrm{X}_{2}$ complexes which in some cases associate further through long halide bridges. For a given diphosphine, the Ge-P distances are essentially independent of halide or whether further long halide bridges are present. There are no reported examples of germanium stibine complexes.

The lithium salt of 2-di-t-butylphosphinomethylhexafluoropropane-2-ol $\left({ }^{t} \mathrm{Bu}_{2} \mathrm{PCH}_{2} \mathrm{C}\left(\mathrm{CF}_{3}\right)_{2} \mathrm{O}^{-} \mathrm{Li}^{+}\right.$, LLi) reacts with $\left[\mathrm{GeCl}_{2}\right.$ (dioxane)] to form $\left[\mathrm{GeL}_{2}\right]$ which contains two anionic $\mathrm{P}, \mathrm{O}-$ donor chelate ligands [279]. Ge(II) complexes of phosphine functionalised $\beta$-diketiminates have also been reported [280].

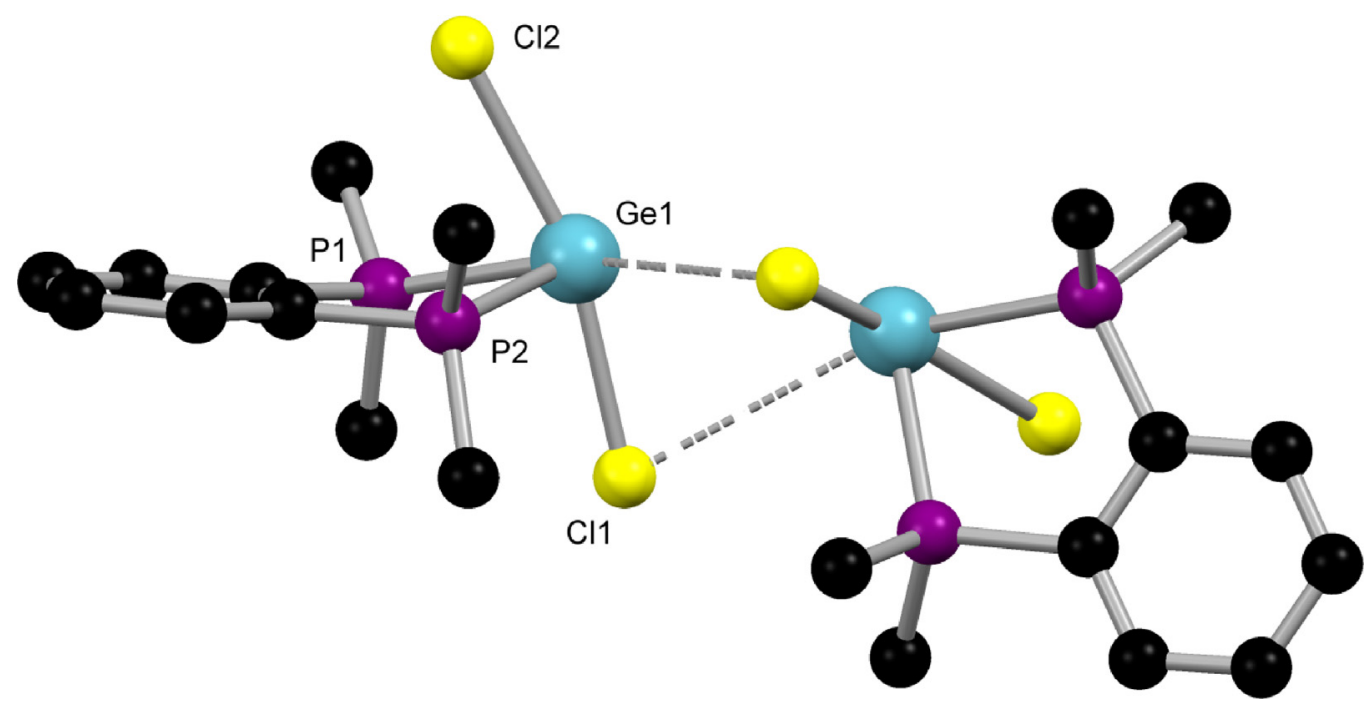

Fig. 46. View of the structure of $\left[\left\{\mathrm{GeCl}_{2}\left\{o-\mathrm{C}_{6} \mathrm{H}_{4}\left(\mathrm{PMe}_{2}\right)_{2}\right\}\right\}_{2}\right]$ redrawn from Ref. [277]. 


\subsection{Tin}

The tin(IV) coordination chemistry of halides and organometallic species is well developed, and the main types of pnictogen ligand complexes, based upon six-coordination, such as $\left[\mathrm{SnX}{ }_{4}\left(\mathrm{PR}_{3}\right)_{2}\right]$, $\left[\mathrm{SnR}_{2}^{\prime} \mathrm{X}_{2}\left(\mathrm{PR}_{3}\right)_{2}\right]$ or their diphosphine analogues, were discussed in previous articles $[7,8]$. Early work mostly used microanalysis and vibrational spectroscopy as the characterisation techniques and there were also several studies using ${ }^{119} \mathrm{Sn}$ Mössbauer spectroscopy. In recent work, multinuclear NMR and X-ray crystallography have taken over as the major characterisation techniques (Tables 11 and 12). In contrast, tin(II) chemistry of neutral Group 15 ligands has received surprisingly little effort, given the extensive work on tin chemistry in general.

$\mathrm{SnF}_{4}$ is an inert polymer, but by reacting $\left[\mathrm{SnF}_{4}(\mathrm{MeCN})_{2}\right]$ with the appropriate phosphine in $\mathrm{CH}_{2} \mathrm{Cl}_{2}$, trans- $\left[\mathrm{SnF}_{4}\left(\mathrm{PR}_{3}\right)_{2}\right](\mathrm{R}=\mathrm{Me}$ or $\mathrm{Cy}$ ) and cis-[SnF 4 (diphosphine)] (diphosphine $=\mathrm{R}_{2} \mathrm{P}\left(\mathrm{CH}_{2}\right)_{2} \mathrm{PR}_{2}$, $\mathrm{R}=\mathrm{Me}, \mathrm{Et}, \mathrm{Ph}$ or $\mathrm{Cy} ; \mathrm{o}-\mathrm{C}_{6} \mathrm{H}_{4}\left(\mathrm{PR}_{2}\right)_{2}, \mathrm{R}=\mathrm{Me}$ or $\left.\mathrm{Ph}\right)$ were obtained [281]. The products are white, moisture sensitive solids, which are also dioxygen sensitive in solution. Multinuclear NMR $\left({ }^{1} \mathrm{H},{ }^{19} \mathrm{~F},{ }^{31} \mathrm{P}\left\{{ }^{1} \mathrm{H}\right\},{ }^{119} \mathrm{Sn}\right)$ spectroscopy provided a large amount of data. For example, the ${ }^{119} \mathrm{Sn}$ spectra of the cis$\left[\mathrm{SnF}_{4}(\right.$ diphosphine $\left.)\right]$ complexes are 27 line multiplets $(t, t, t)$ from which the appropriate coupling constants are readily extracted. The cis- $\left[\mathrm{SnX}_{4}\left\{\mathrm{Et}_{2} \mathrm{P}\left(\mathrm{CH}_{2}\right)_{2} \mathrm{PEt}_{2}\right\}\right](\mathrm{X}=\mathrm{F}$ or $\mathrm{Cl})$ are isomorphous and the Sn-P distance is significantly shorter in the fluoride $(2.606(1) \AA)$ than in cis-[SnCl $\left.\left.4 \mathrm{Et}_{2} \mathrm{P}\left(\mathrm{CH}_{2}\right)_{2} \mathrm{PEt}_{2}\right\}\right](2.648(2) \AA)$. The longer distance in trans-[ $\left.\mathrm{SnF}_{4}\left(\mathrm{PCy}_{3}\right)_{2}\right](2.654(1) \AA)$ may well be largely down to the steric bulk of the phosphine. Attempts to isolate arsine complexes of $\mathrm{SnF}_{4}$ were unsuccessful [281].

The other three $\operatorname{tin}(\mathrm{IV})$ halides, $\mathrm{SnX}_{4}(\mathrm{X}=\mathrm{Cl}, \mathrm{Br}$ or I), are tetrahedral monomers, and synthesis of their phosphine or arsine complexes (there are no reports of stibines) mostly involve mixing the $\mathrm{SnX}_{4}$ with the ligand under anhydrous conditions and an inert atmosphere in $\mathrm{CH}_{2} \mathrm{Cl}_{2}$, hexane, benzene etc. $[281,282,285-287,292]$. For tertiary phosphines the six-coordinate trans-[ $\left.\mathrm{SnX}_{4}\left(\mathrm{PR}_{3}\right)_{2}\right]$ are the usual solid products, exemplified by those of $\mathrm{PMe}_{3}$ [284,287], $\mathrm{PEt}_{3}$ [282], $\mathrm{PPh}_{3}, \mathrm{PMe}_{2} \mathrm{Ph}$ and $\mathrm{P}^{n} \mathrm{Bu}_{3}$ [292], although NMR spectroscopy often identifies a mixture of cis and trans isomers in solution. $\mathrm{AsPh}_{3}$ complexes of both $1: 1$ and $2: 1$

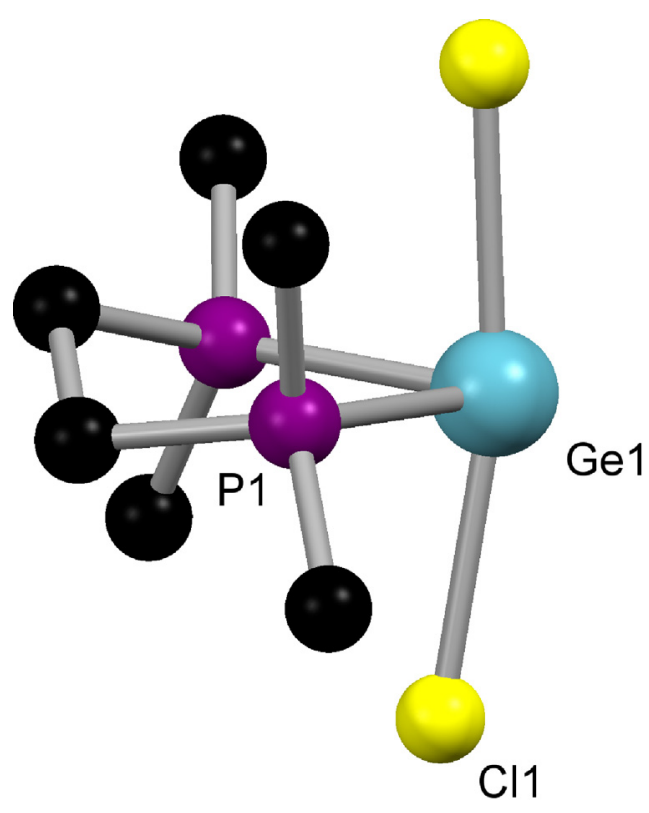

Fig. 48. View of the structure of $\left[\mathrm{GeCl}_{2}\left\{\mathrm{Me}_{2} \mathrm{P}\left(\mathrm{CH}_{2}\right)_{2} \mathrm{PMe}_{2}\right\}\right]$ redrawn from Ref. [277].

stoichiometry have been reported [285,294]. Interestingly, Mahon et al. [285] determined the X-ray structures of $\left[\mathrm{SnX}_{4}\left(\mathrm{AsPh}_{3}\right)_{2}\right.$ ], finding that the chloride was the expected six-coordinate trans isomer (Fig. 49), but the bromide was trigonal bipyramidal [ $\left.\mathrm{SnBr}_{4}\left(\mathrm{AsPh}_{3}\right)\right]$ with a second uncoordinated $\mathrm{AsPh}_{3}$ group in the lattice interacting with the axial $\mathrm{Br}$ group (Fig. 50).

A different synthetic approach involved the reaction of tin powder with $\mathrm{PR}_{3} \mathrm{X}_{2}\left(\mathrm{R}={ }^{n} \mathrm{Pr}, \mathrm{X}=\mathrm{I} ; \mathrm{R}_{3}=\mathrm{Ph}, \mathrm{Ph}_{2} \mathrm{Me}\right.$ or $\mathrm{PhMe}_{2}$, $\mathrm{X}=\mathrm{Br}$ or I) in diethyl ether [283,293], which produced the first structurally authenticated phosphine adduct of tin(IV) iodide, trans- $\left[\mathrm{SnI}_{4}\left(\mathrm{P}^{n} \mathrm{Pr}_{3}\right)_{2}\right]$, and cis/trans mixtures for $\left[\mathrm{SnBr}_{4}\left(\mathrm{PR}_{3}\right)_{2}\right]$ identified by a combination of vibrational and Mössbauer spectroscopy. The $\mathrm{PR}_{3} \mathrm{I}_{2}\left(\mathrm{R}_{3}=\mathrm{Ph}, \mathrm{Ph}_{2} \mathrm{Me}\right)$ reactions produced mixtures containing cis/trans-[ $\left.\mathrm{SnI}_{4}\left(\mathrm{PR}_{3}\right)_{2}\right]$ and $\left[\mathrm{PR}_{3} \mathrm{I}\right]\left[\mathrm{SnI}_{3}\right]$. The direct reaction of $\mathrm{SnX}_{4}(\mathrm{X}=\mathrm{Cl}$ or $\mathrm{Br})$ with $\mathrm{P}^{t} \mathrm{Bu}_{3}$ results in formation of

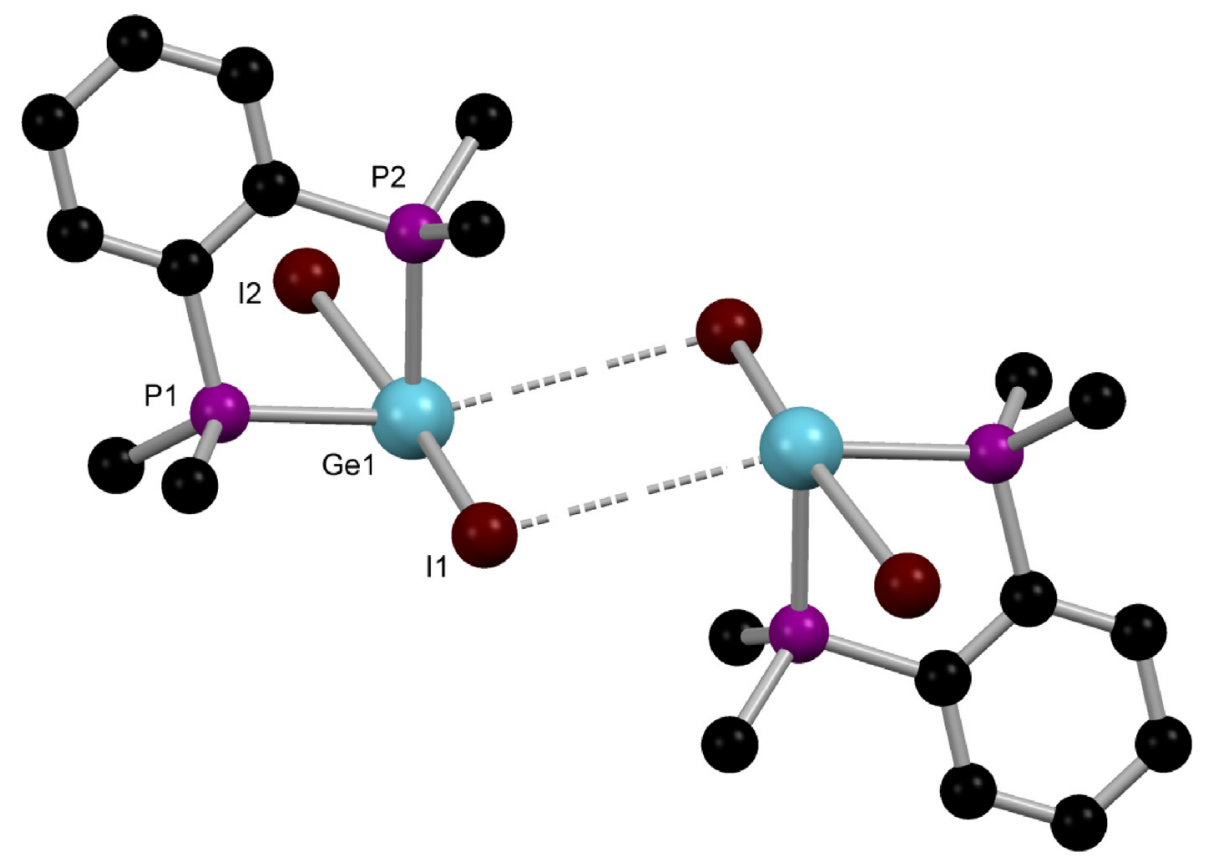

Fig. 47. View of the structure of $\left[\left\{\mathrm{GeI}_{2}\left\{0-\mathrm{C}_{6} \mathrm{H}_{4}\left(\mathrm{PMe}_{2}\right)_{2}\right\}\right\}_{2}\right]$ redrawn from Ref. [277]. 
Table 11

X-ray structures of tin complexes.

\begin{tabular}{|c|c|c|c|c|}
\hline Complex & Sn-P/As $(\AA)$ & Sn-X $(\AA)$ & Comments & Ref \\
\hline trans- $\left[\mathrm{SnF}_{4}\left(\mathrm{PCy}_{3}\right)_{2}\right]$ & $2.6538(11)$ & $1.959(2), 1.980(2)$ & & [281] \\
\hline trans $-\left[\mathrm{SnCl}_{4}\left(\mathrm{PEt}_{3}\right)_{2}\right]$ & $2.615(5)$ & $2.445(5), 2.455(5)$ & & [282] \\
\hline trans- $\left[\mathrm{SnI}_{4}\left(\mathrm{P}^{n} \mathrm{Pr}_{3}\right)_{2}\right]$ & $2.69(1)$ & $2.863(3), 2.872(3)$ & & [283] \\
\hline trans- $\left[\mathrm{SnCl}_{4}\left(\mathrm{PMe}_{3}\right)_{2}\right]$ & $2.5654(7)$ & $2.4762(6), 2.4565(7)$ & & [284] \\
\hline$\left[\mathrm{SnCl}_{3}\left(\mathrm{PMe}_{3}\right)_{2}\right]\left[\mathrm{AlCl}_{4}\right]$ & $2.5439(12), 2.5347(12)$ & $2.3490(12), 2.3955(10)$ & trans Tbp & [284] \\
\hline$\left[\mathrm{SnCl}_{2}\left(\mathrm{PMe}_{3}\right)_{2}\right]\left[\mathrm{AlCl}_{4}\right]_{2}$ & $2.5390(6), 2.5414(6)$ & $2.3367(5), 2.3575(5)$ & & [284] \\
\hline trans- $\left[\mathrm{SnCl}_{4}\left(\mathrm{AsPh}_{3}\right)_{2}\right]$ & $2.7623(3)$ & $2.4153(8), 2.4239(7)$ & & [285] \\
\hline$\left[\mathrm{SnBr}_{4}\left(\mathrm{AsPh}_{3}\right)\right] \cdot \mathrm{AsPh}_{3}$ & $2.780(1)$ & $2.484(2), 2.486(2)$ & Tbp axial As & [285] \\
\hline$\left[\mathrm{SnCl}_{4}\left\{\boldsymbol{\kappa}^{1}-\mathrm{Ph}_{2} \mathrm{PCH}_{2} \mathrm{PPh}_{2}\right\}_{2}\right]$ & $2.649(1)$ & $2.429(1), 2.450(1)$ & & [290] \\
\hline$\left[\mathrm{SnF}_{4}\left\{\mathrm{Et}_{2} \mathrm{P}\left(\mathrm{CH}_{2}\right)_{2} \mathrm{PEt}_{2}\right\}\right]$ & $2.6058(9)$ & $1.9532(16), 1.9863(17)$ & & [281] \\
\hline$\left[\mathrm{SnCl}_{4}\left\{\mathrm{Et}_{2} \mathrm{P}\left(\mathrm{CH}_{2}\right)_{2} \mathrm{PEt}_{2}\right\}\right]$ & $2.6481(17)$ & $2.4084(14), 2.4529(14)$ & & [281] \\
\hline$\left[\mathrm{SnCl}_{4}\left\{\mathrm{Ph}_{2} \mathrm{P}\left(\mathrm{CH}_{2}\right)_{2} \mathrm{PPh}_{2}\right\}\right]$ & $2.653(2), 2.679(2)$ & $2.402(2)-2.447(2)$ & & [286] \\
\hline$\left[\mathrm{SnCl}_{4}\left\{\right.\right.$ cis $\left.\left.-\mathrm{Ph}_{2} \mathrm{PCH}=\mathrm{CHPPh}_{2}\right\}\right]$ & $2.6537(5), 2.6780(5)$ & $2.3745(6)-2.4138(5)$ & & [291] \\
\hline$\left[\mathrm{SnI}_{4}\left\{0-\mathrm{C}_{6} \mathrm{H}_{4}\left(\mathrm{AsMe}_{2}\right)_{2}\right\}\right]$ & $2.716(2), 2.752(2)$ & $2.787(2)-2.859(2)$ & & [287] \\
\hline$\left[\mathrm{SnCl}_{5}\left\{\mathrm{Ph}_{2}(\mathrm{H}) \mathrm{P}\left(\mathrm{CH}_{2}\right)_{2} \mathrm{PPh}_{2}\right\}\right]$ & $2.7312(7), 2.7573(6)$ & $2.3986(6)-2.4369(6)$ & Zwitterion & [288] \\
\hline$\left[\mathrm{SnBr}_{4}\left\{\mathrm{Ph}_{2} \mathrm{As}\left(\mathrm{CH}_{2}\right)_{2} \mathrm{P}(\mathrm{O}) \mathrm{Ph}_{2}\right\}\right]$ & $2.7519(4)$ & $2.5392(4)-2.5856(4)$ & As, $\mathrm{O}$ coord & [288] \\
\hline$\left[\mathrm{SnBr}_{4}\left\{\mathrm{MeC}\left(\mathrm{CH}_{2} \mathrm{AsMe}_{2}\right)_{3}\right\}\right]$ & $2.6932(5), 2.7095(6)$ & $2.5479(5)-2.5807(5)$ & $\mathrm{\kappa}^{2}$ Coord triarsine & [281] \\
\hline$\left[\mathrm{SnCl}_{3}{ }^{n} \mathrm{Bu}\left(\mathrm{PPh}_{3}\right)\right]$ & $2.862(2)$ & $2.336(2)-2.404(2)$ & & [289] \\
\hline$\left[\mathrm{SnCl}_{3} \mathrm{Ph}\left\{0-\mathrm{C}_{6} \mathrm{H}_{4}(\mathrm{PPhMe})_{2}\right\}\right]$ & $2.659(1), 2.747(1)$ & $2.445(1)-2.503(1)$ & & [290] \\
\hline$\left[\mathrm{SnCl}_{3} \mathrm{Me}\left\{c i s-\mathrm{Ph}_{2} \mathrm{PCH}=\mathrm{CHPPh}_{2}\right\}\right]$ & $2.6686(5), 2.7578(5)$ & $2.4219(5)-2.5053(5)$ & & [291] \\
\hline$\left[\mathrm{SnCl}_{3}{ }^{n} \mathrm{Bu}\left\{\right.\right.$ cis- $\left.\left.\mathrm{Ph}_{2} \mathrm{PCH}=\mathrm{CHPPh}_{2}\right\}\right]$ & $2.6945(7), 2.7526(7)$ & $2.4360(8)-2.4817(7)$ & & [291] \\
\hline$\left[\mathrm{SnCl}_{3} \mathrm{Ph}\left\{\right.\right.$ cis- $\left.\left.\mathrm{Ph}_{2} \mathrm{PCH}=\mathrm{CHPPh}_{2}\right\}\right]$ & 2.7036(13), 2.7231(13) & $2.4233(12)-2.4962(13)$ & & [291] \\
\hline$\left[\mathrm{SnClMe}_{2}\left\{{ }^{\mathrm{t}} \mathrm{Bu}(\mathrm{Ph}) \mathrm{P}\left(\mathrm{CH}_{2}\right)_{3}\right\}\right]$ & $3.078(2)$ & $2.494(2)$ & Tbp $\mathrm{C}_{3} \mathrm{PCl}$ coord & [306] \\
\hline$\left[\mathrm{SnCl}_{2}\left\{\mathrm{Ph}_{2} \mathrm{P}\left(\mathrm{CH}_{2}\right)_{3}\right\}_{2}\right]$ & $3.086(2)$ & $2.134(6), 2.487(2)$ & 6-Coord $\mathrm{P}_{2} \mathrm{C}_{2} \mathrm{Cl}_{2}$ & [307] \\
\hline$\left[\left\{\mathrm{SnCl}_{3}\left\{\mathrm{Ph}_{2} \mathrm{P}\left(\mathrm{CH}_{2}\right)_{2}\right\}\right\}_{2}\right]$ & $2.605(2)$ & $2.389(2)-2.620(2)$ & Cl-bridged dimer & [307] \\
\hline$\left[\left\{\mathrm{SnCl}_{3}\left\{\mathrm{Ph}_{2} \mathrm{P}\left(\mathrm{CH}_{2}\right)_{3}\right\}\right\}_{2}\right]$ & $2.686(1)$ & $2.377(1)-2.826(1)$ & Cl-bridged dimer & [307] \\
\hline$\left[\left\{\mathrm{SnCl}_{2} \mathrm{Me}\left\{\mathrm{Ph}_{2} \mathrm{P}\left(\mathrm{CH}_{2}\right)_{3}\right\}\right\}_{2}\right]$ & $2.930(2)$ & $2.409(1), 2.516(2)$ & Cl-bridged dimer & [307] \\
\hline$\left[\mathrm{SnCl}_{2}\left\{\mathrm{o}-\mathrm{C}_{6} \mathrm{H}_{4}\left(\mathrm{PPh}_{2}\right)_{2}\right\}\right]$ & $2.8293(9)$ & $2.4541(9), 2.5536(11)$ & Dimer $\kappa^{1}$-Diphosphine & [309] \\
\hline$\left[\mathrm{SnCl}_{2}\left\{0-\mathrm{C}_{6} \mathrm{H}_{4}\left(\mathrm{PMe}_{2}\right)_{2}\right\}\right]$ & $2.692(4), 2.657(3)$ & $2.673(3), 2.743(4)$ & Dimer 5-coord & [309] \\
\hline$\left[\mathrm{SnCl}_{2}\left\{\mathrm{Me}_{2} \mathrm{P}\left(\mathrm{CH}_{2}\right)_{2} \mathrm{PMe}_{2}\right\}\right]$ & 2.6502(15), 2.6950(14) & $2.6896(14), 2.7292(14)$ & Dimer 5-coord & [309] \\
\hline$\left[\left(\mathrm{SnCl}_{2}\right)_{2}\left\{\mu-\mathrm{Ph}_{2} \mathrm{P}\left(\mathrm{CH}_{2}\right)_{2} \mathrm{PPh}_{2}\right\}\right]$ & $2.7675(5)$ & $2.4808(5), 2.5163(5)$ & Ligand bridged & [309] \\
\hline$\left[\mathrm{SnCl}\left\{0-\mathrm{C}_{6} \mathrm{H}_{4}\left(\mathrm{AsMe}_{2}\right)_{2}\right\}\right]\left[\mathrm{SnCl}_{3}\right]$ & $2.7986(7)$ & $2.5623(13)$ & Trigonal pyramid & [309] \\
\hline$\left[\mathrm{SnCl}\left\{\mathrm{Ph}_{2} \mathrm{PCH}_{2}\left(2-\mathrm{C}_{5} \mathrm{H}_{4} \mathrm{~N}\right)\right\}\right]\left[\mathrm{SnCl}_{3}\right]$ & $2.6962(5)$ & $2.4405(5)$ & $\mathrm{NPCl}$ coord. cation & [313] \\
\hline$\left[\mathrm{Sn}\left\{{ }^{t} \mathrm{Bu}_{2} \mathrm{PCH}_{2} \mathrm{C}\left(\mathrm{CF}_{3}\right)_{2} \mathrm{O}\right\}_{2}\right]$ & $2.8376(12), 2.8460(11)$ & & & [279] \\
\hline$\left[\mathrm{Sn}\left\{\mathrm{PhP}\left(\mathrm{CH}_{2}\right)_{2} \mathrm{~S}\right\}_{2}\right]$ & $2.614(5)$ & & & [314] \\
\hline
\end{tabular}

$\left[\mathrm{P}^{t} \mathrm{Bu}_{3} \mathrm{X}\right]\left[\mathrm{SnX} \mathrm{X}_{3}\right]$ analogous to the redox germanium chemistry discussed in Section 7.2 [267,269]. Cationic complexes form on treating trans-[ $\left.\mathrm{SnCl}_{4}\left(\mathrm{PMe}_{3}\right)_{2}\right]$ with $\mathrm{AlCl}_{3}$, one equivalent giving $\left[\mathrm{SnCl}_{3}\left(\mathrm{PMe}_{3}\right)_{2}\right]\left[\mathrm{AlCl}_{4}\right]$, and an excess, $\left[\mathrm{SnCl}_{2}\left(\mathrm{PMe}_{3}\right)_{2}\right]\left[\mathrm{AlCl}_{4}\right]_{2}$ [284]. The former is a trigonal bipyramid with axial phosphines (Fig. 51(a)), and the latter a very distorted octahedron with axial phosphines, two short $\mathrm{Sn}-\mathrm{Cl}$ bonds and two longer $\mathrm{Sn}-\mathrm{Cl}$ contacts to chloroaluminate anions (Fig. 51(b)). The stability of tin(IV) halide phosphines falls $\mathrm{F} \sim \mathrm{Cl}>\mathrm{Br}>\mathrm{I}$, although with arsines the order is partially reversed $\mathrm{F}<<\mathrm{Cl}>\mathrm{Br}>\mathrm{I}[261,287]$.

Most of the synthesis papers report vibrational spectrocopic data for $\mathrm{SnX}_{4}$ complexes [281,285,287,293,295]. Multinuclear

Table 12

Illustrative ${ }^{119} \mathrm{Sn}$ and ${ }^{31} \mathrm{P}\left\{{ }^{1} \mathrm{H}\right\}$ NMR data of tin complexes [281,287,291,296,297,309].

\begin{tabular}{|c|c|c|c|c|}
\hline Complex & $\delta\left({ }^{119} \mathrm{Sn}\right)^{\mathrm{a}}$ & $\delta\left({ }^{31} \mathrm{P}\right)^{\mathrm{b}}$ & $\Delta(\mathrm{P})^{\mathrm{c}}$ & ${ }^{1} J\left({ }^{119} \mathrm{Sn}-{ }^{31} \mathrm{P}\right)(\mathrm{Hz})^{\mathrm{d}}$ \\
\hline trans- $\left[\mathrm{SnF}_{4}\left(\mathrm{PMe}_{3}\right)_{2}\right]$ & -628 & -19.1 & +43 & 2975 \\
\hline trans- $\left[\mathrm{SnCl}_{4}\left(\mathrm{PMe}_{3}\right)_{2}\right]$ & -646 & 8.0 & +70 & 2768 \\
\hline trans- $\left[\mathrm{SnBr}_{4}\left(\mathrm{PMe}_{3}\right)_{2}\right]$ & N.o. & -3.0 & +59 & N.o. \\
\hline trans $-\left[\mathrm{SnI}_{4}\left(\mathrm{PMe}_{3}\right)_{2}\right]$ & N.o. & -3.7 & +58 & N.o. \\
\hline trans- $\left[\mathrm{SnCl}_{4}\left(\mathrm{PEt}_{3}\right)_{2}\right]$ & -572 & +20.9 & +42 & 2391 \\
\hline trans- $\left[\mathrm{SnBr}_{4}\left(\mathrm{PEt}_{3}\right)_{2}\right]$ & -910 & +2.4 & +23 & 1947 \\
\hline$\left[\mathrm{SnF}_{4}\left\{0-\mathrm{C}_{6} \mathrm{H}_{4}\left(\mathrm{PMe}_{2}\right)_{2}\right\}\right]$ & -657.6 & -37.8 & +17 & 1520 \\
\hline$\left[\mathrm{SnF}_{4}\left\{0-\mathrm{C}_{6} \mathrm{H}_{4}\left(\mathrm{PPh}_{2}\right)_{2}\right\}\right]$ & -665.9 & -18.9 & -6 & 1520 \\
\hline$\left[\mathrm{SnCl}_{4}\left\{0-\mathrm{C}_{6} \mathrm{H}_{4}\left(\mathrm{PPh}_{2}\right)_{2}\right\}\right]$ & -607.5 & -13.0 & $\sim 0$ & 890 \\
\hline$\left[\mathrm{SnBr}_{4}\left\{0-\mathrm{C}_{6} \mathrm{H}_{4}\left(\mathrm{PPh}_{2}\right)_{2}\right\}\right]$ & -1218 & -24.2 & -11 & 305 \\
\hline$\left[\mathrm{SnI}_{4}\left\{\mathrm{o}-\mathrm{C}_{6} \mathrm{H}_{4}\left(\mathrm{PPh}_{2}\right)_{2}\right\}\right]$ & N.o. & -52.5 & -39 & N.o. \\
\hline$\left[\mathrm{SnF}_{4}\left\{\mathrm{Et}_{2} \mathrm{P}\left(\mathrm{CH}_{2}\right)_{2} \mathrm{PEt}_{2}\right\}\right]$ & -649 & -11.6 & +6 & 1628 \\
\hline$\left[\mathrm{SnCl}_{4}\left\{\mathrm{Me}_{2} \mathrm{P}\left(\mathrm{CH}_{2}\right)_{2} \mathrm{PMe}_{2}\right\}\right]$ & -617 & -20.9 & +26 & 1080 \\
\hline$\left[\mathrm{SnBr}_{4}\left\{\mathrm{Me}_{2} \mathrm{P}\left(\mathrm{CH}_{2}\right)_{2} \mathrm{PMe}_{2}\right\}\right]$ & -1213 & -26.0 & +21 & 680 \\
\hline$\left[\mathrm{SnI}_{4}\left\{\mathrm{Me}_{2} \mathrm{P}\left(\mathrm{CH}_{2}\right)_{2} \mathrm{PMe}_{2}\right\}\right]$ & -2425 & -40 & +7 & $\sim 200$ \\
\hline$\left[\mathrm{SnCl}_{3} \mathrm{Me}\left\{\right.\right.$ cis $\left.\left.-\mathrm{Ph}_{2} \mathrm{PCH}=\mathrm{CHPPh}_{2}\right\}\right]$ & -462.5 & $-24.1,-31.3$ & $-2,-9$ & 2429 \\
\hline$\left[\mathrm{SnCl}_{3}{ }^{n} \mathrm{Bu}\left\{c i s-\mathrm{Ph}_{2} \mathrm{PCH}=\mathrm{CHPPh}_{2}\right\}\right]$ & -456.3 & $-23.1,-30.2$ & $-1,-8$ & 2344 \\
\hline$\left[\mathrm{SnCl}_{3} \mathrm{Ph}\left\{\right.\right.$ cis- $\left.\left.\mathrm{Ph}_{2} \mathrm{PCH}=\mathrm{CHPPh}_{2}\right\}\right]$ & -510.1 & $-25.0,-32.5$ & $-3,-10$ & 2327 \\
\hline$\left[\mathrm{SnCl}_{2}\left\{0-\mathrm{C}_{6} \mathrm{H}_{4}\left(\mathrm{PMe}_{2}\right)_{2}\right\}\right]$ & N.o. & -27.9 & +27 & 948 \\
\hline$\left[\mathrm{SnCl}_{2}\left\{\mathrm{Me}_{2} \mathrm{P}\left(\mathrm{CH}_{2}\right)_{2} \mathrm{PMe}_{2}\right\}\right]$ & N.o. & +8.4 & +55 & 2005 \\
\hline
\end{tabular}

a Relative to $\mathrm{SnMe}_{4}$.

b Relative to $\mathrm{H}_{3} \mathrm{PO}_{4}$.

c Coordination shift.

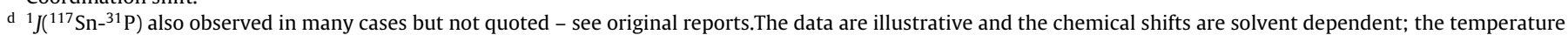
of data collection is also not indicated here. 


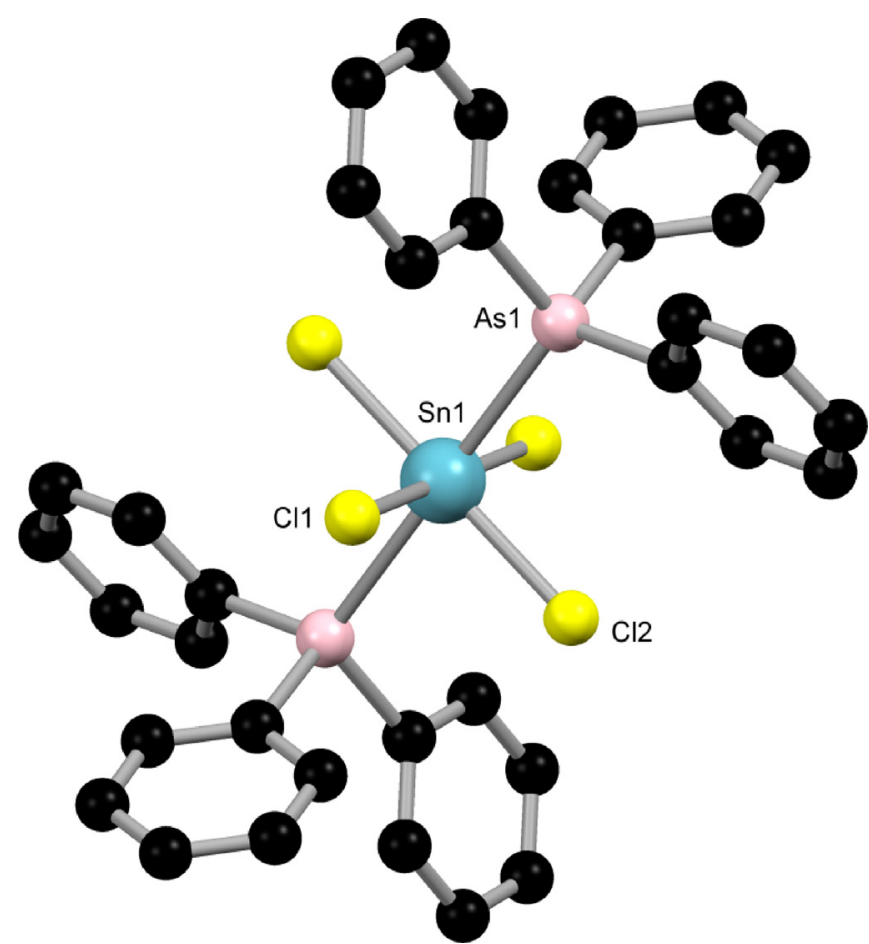

Fig. 49. View of the structure of $\left[\mathrm{SnCl}_{4}\left(\mathrm{AsPh}_{3}\right)_{2}\right]$ redrawn from Ref. [285].

NMR data has also been reported for many tin(IV) complexes and some typical examples are shown in Table 12 $[281,287,291,292,296-298]$. The ${ }^{119}$ Sn chemical shifts are surprisingly similar for corresponding complexes of $\mathrm{SnF}_{4}$ and $\mathrm{SnCl}_{4}$, but those of $\mathrm{SnBr}_{4}$ and $\mathrm{SnI}_{4}$ show much more deshielded resonances. Few values have been recorded for the iodo-complexes, even at low temperatures, due to the extensive dissociation in solution. The ${ }^{1} J\left({ }^{119} \mathrm{Sn}-{ }^{31} \mathrm{P}\right)$ coupling constants fall with halogen $\mathrm{F}>\mathrm{Cl}>\mathrm{Br}>\mathrm{I}$ and are greater for trans $\mathrm{P}-\mathrm{Sn}-\mathrm{P}$ than for cis $\mathrm{P}-\mathrm{Sn}-\mathrm{P}$ arrangements.

Diphosphine complexes are usually six-coordinate monomers with a cis-geometry (Table 11 ); typically the $\mathrm{Sn}-\mathrm{X}_{\text {transx }}$ bonds

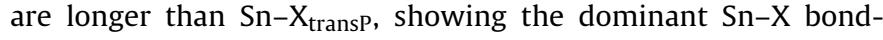
ing $[281,286,287,291]$. The diphosphine $\mathrm{Ph}_{2} \mathrm{PCH}_{2} \mathrm{PPh}_{2}$, which has a short backbone, gave trans- $\left[\mathrm{SnCl}_{4}\left\{\kappa^{1}-\mathrm{Ph}_{2} \mathrm{PCH}_{2} \mathrm{PPh}_{2}\right\}_{2}\right]$ [290]. Crystals were serendipitously obtained of the zwitterionic [ $\left.\mathrm{SnCl}_{5}\left\{\mathrm{Ph}_{2} \mathrm{P}\left(\mathrm{CH}_{2}\right)_{2} \mathrm{P}(\mathrm{H}) \mathrm{Ph}_{2}\right\}\right]$ [288] (Fig. 52). Diarsine complexes are rarer, although [ $\left.\mathrm{SnI}_{4}\left\{0-\mathrm{C}_{6} \mathrm{H}_{4}\left(\mathrm{AsMe}_{2}\right)_{2}\right\}\right]$ [287] is one of the very few structurally characterised $\mathrm{SnI}_{4}$ adducts with neutral ligands. Few attempts to make complexes of $\mathrm{SnX}_{4}$ with polydentate ligands have been reported, presumably because only two coordination sites on tin are available, and therefore higher ligand denticity offers nothing new. One example is $\left[\mathrm{SnBr}_{4}\left\{\mathrm{MeC}\left(\mathrm{CH}_{2} \mathrm{AsMe}_{2}\right)_{3}\right\}\right]$, where the tripodal triarsine is $\mathrm{K}^{2}$-coordinated to the tin (Fig. 53) [281].

The tin(IV) halide phosphine complexes are very moisture sensitive and also dioxygen sensitive in solution. Whilst dioxygen sensitivity is of course found for alkylphosphines themselves, tin(IV) halides promote the oxidation of air-stable phosphines and diphosphines, although the mechanism is unclear. For $\mathrm{SnF}_{4}$ the reaction is stoichiometric since the phosphine oxide or diphosphine dioxide formed coordinates very strongly to the tin, stopping further reaction, whereas with the extensively dissociated adducts of $\mathrm{SnI}_{4}$ the reaction can be made catalytic $[261,299,300]$. Use of ${ }^{18} \mathrm{O}_{2}$ resulted in incorporation of the ${ }^{18} \mathrm{O}$ label into the phosphine oxide, confirming the reaction proceeds via reaction with dioxygen rather than by a hydrolysis mechanism [299,300]. However, tertiary arsines are not oxidised under similar conditions, reflecting the much reduced stability of $A s(V)$ versus $P(V)$, a notable illustration of which is the crystallographic identification of $\left[\mathrm{SnBr}_{4}\left\{\mathrm{Ph}_{2} \mathrm{As}\left(\mathrm{CH}_{2}\right)_{2} \mathrm{P}(\mathrm{O}) \mathrm{Ph}_{2}\right\}\right]$ formed by adventitious oxidation of $\mathrm{Ph}_{2} \mathrm{As}\left(\mathrm{CH}_{2}\right)_{2} \mathrm{PPh}_{2}$ [288].

Organotin halides $\mathrm{SnR}_{4-n} \mathrm{X}_{n}(n=3-1)$ also form phosphine adducts [289-291,298,301-304]. The Lewis acidity falls with increase in the number of R groups, and is less than that of $\mathrm{SnX}_{4}$; as a result both five- and six-coordinate complexes are formed depending upon the phosphine and the organotin species involved. The $\mathrm{Ph}_{2} \mathrm{P}\left(\mathrm{CH}_{2}\right)_{n} \mathrm{PPh}_{2}(n=1$ or 2$)$ complexes of $\mathrm{SnPh}_{2} \mathrm{X}_{2}(\mathrm{X}=\mathrm{Cl}$ or $\mathrm{Br})$ are clearly five-coordinate in solution, with fast exchange occurring between the free and coordinated phosphine groups on the NMR time-scale [290]. However, using more rigid diphosphines such as cis- $\mathrm{Ph}_{2} \mathrm{PCH}=\mathrm{CHPPh}_{2}$, or the chiral $o-\mathrm{C}_{6} \mathrm{H}_{4}(\mathrm{PPhMe})_{2}$, produces complexes that are six-coordinate both in the solid state and in solution at low temperatures [290,291]. Disproportionation of $\mathrm{RSnX}_{3}$ into

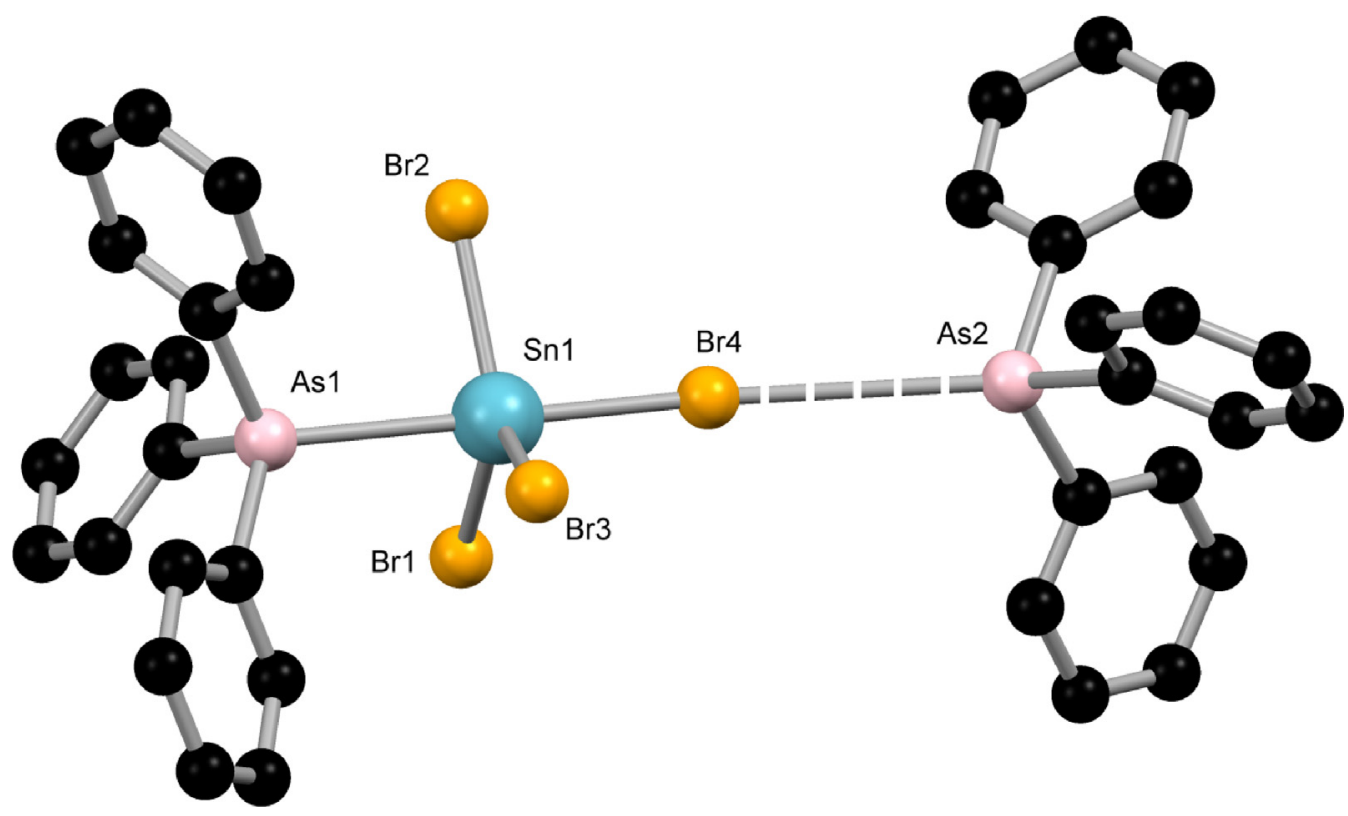

Fig. 50. View of the structure of $\left[\mathrm{SnBr}_{4}\left(\mathrm{AsPh}_{3}\right)\right] . \mathrm{AsPh}_{3}$ redrawn from Ref. [285]. 
(a)
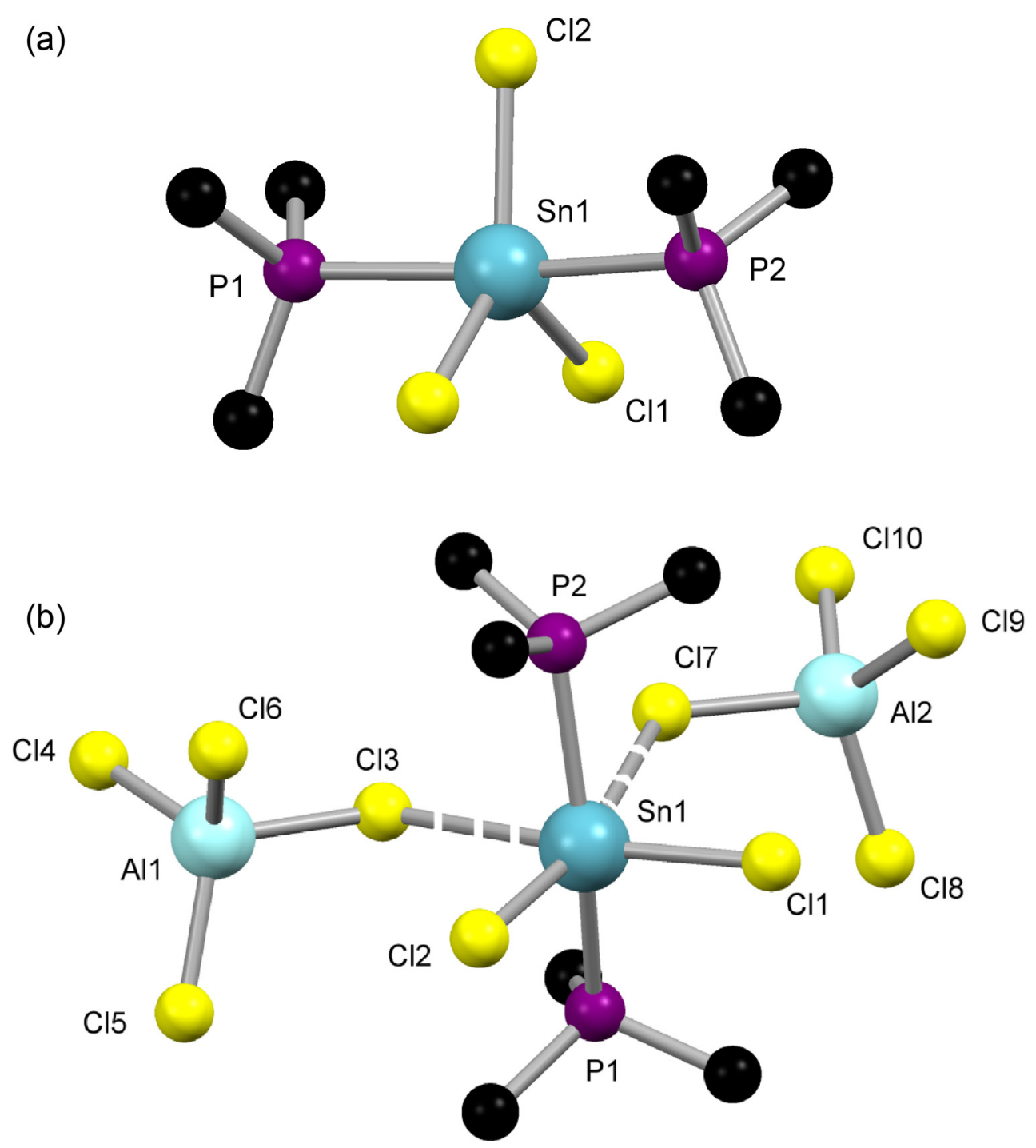

Fig. 51. (a) View of the structure of the cation $\left[\mathrm{SnCl}_{3}\left(\mathrm{PMe}_{3}\right)_{2}\right]^{+}$redrawn from Ref. [284]. (b) View of the structure of [SnCl $\left.2\left(\mathrm{PMe}_{3}\right)_{2}\right]\left[\mathrm{AlCl}_{4}\right]_{2}$ redrawn from Ref. [284].

$\mathrm{R}_{2} \mathrm{SnX}_{2}$ and $\mathrm{SnX}_{4}$ upon reaction with phosphines was also observed in some systems [290,291,302].

Intramolecular coordination of P-functionalised organotin halides produces more stable complexes [305-308]. For example, chloro(3-(t-butyl(phenyl)phosphino)propyl)dimethyltin is trigonal bipyramidal with apical Cl,P [306], [ $\left.\mathrm{SnCl}_{2}\left\{\mathrm{Ph}_{2} \mathrm{P}\left(\mathrm{CH}_{2}\right)_{3}\right\}_{2}\right]$ is a six-coordinate monomer and $\left[\left\{\mathrm{SnCl}_{2} \mathrm{R}^{\prime}\left\{\mathrm{Ph}_{2} \mathrm{P}\left(\mathrm{CH}_{2}\right)_{3}\right\}\right\}_{2}\right]\left(\mathrm{R}^{\prime}=\mathrm{Cl}\right.$ or $\mathrm{Me})$ and $\left[\left\{\mathrm{SnCl}_{3}\left\{\mathrm{Ph}_{2} \mathrm{P}\left(\mathrm{CH}_{2}\right)_{2}\right\}\right\}_{2}\right]$ are dimers (Fig. 54) [307].

Attempts to prepare phosphine complexes of $\mathrm{SnF}_{2}$ either by direct reaction with a suspension of $\mathrm{SnF}_{2}$ in $\mathrm{MeCN}$, or by displacement of dmso from [ $\mathrm{SnF}_{2}(\mathrm{dmso})$ ] failed [309]. In $\mathrm{CH}_{2} \mathrm{Cl}_{2}$ solution, mixtures of products slowly formed which were mainly phosphonium salts from reaction of the phosphine with the solvent. Similarly, $\mathrm{SnCl}_{2}$ and $\mathrm{PMe}_{3}$ in $\mathrm{CH}_{2} \mathrm{Cl}_{2}$ gave phosphonium cations of the types [ $\left.\mathrm{PMe}_{3} \mathrm{CH}_{2} \mathrm{Cl}\right]^{+}$and $\left[\mathrm{CH}_{2}\left(\mathrm{PMe}_{3}\right)_{2}\right]^{2+}$ [309]. [ $\left.\mathrm{SnX}_{2}\left(\mathrm{P}^{t} \mathrm{Bu}_{3}\right)\right]$ and $\left[\mathrm{SnX} \mathrm{X}_{2}\left\{\mathrm{P}\left(\mathrm{NMe}_{2}\right)_{3}\right\}\right](\mathrm{X}=\mathrm{Cl}$ or $\mathrm{Br})$ have been isolated, and were suggested to be pyramidal monomers from spectroscopic data [310]. Diphosphines similarly failed to complex with $\mathrm{SnF}_{2}$ (which may be due to the absence of an appropriate synthon that would avoid the use of the polymeric and unreactive fluoride itself). However, with $\mathrm{SnCl}_{2}$ a series of 1:1 complexes was isolated; although they differ in structural detail they are reminiscent of the $\mathrm{GeCl}_{2}$ adducts described in Section 7.2. The bulky $0-\mathrm{C}_{6} \mathrm{H}_{4}\left(\mathrm{PPh}_{2}\right)_{2}$ gave $\left[\mathrm{SnCl}_{2}\left\{0-\mathrm{C}_{6} \mathrm{H}_{4}\left(\mathrm{PPh}_{2}\right)_{2}\right\}\right]$ which contained a pyramidal $\mathrm{PCl}_{2}$ core with the second phosphino group $\sim 3.29 \AA$ from the tin, whereas $\left[\mathrm{SnCl}_{2}\left\{0-\mathrm{C}_{6} \mathrm{H}_{4}\left(\mathrm{PMe}_{2}\right)_{2}\right\}\right]$ and [ $\mathrm{SnCl}_{2}\left\{\mathrm{Me}_{2} \mathrm{P}\left(\mathrm{CH}_{2}\right)_{2} \mathrm{PMe}_{2}\right\}$ ] have a $\mathrm{P}_{2} \mathrm{Cl}_{2}$ core (Fig. 55); all are weakly associated into dimers via chlorine bridges [309]. The product of reacting $\mathrm{SnCl}_{2}$ and $\mathrm{Ph}_{2} \mathrm{P}\left(\mathrm{CH}_{2}\right)_{2} \mathrm{PPh}_{2}$, irrespective of the ratio of reactants used, is [( $\left.\left.\mathrm{SnCl}_{2}\right)_{2}\left\{\mu-\mathrm{Ph}_{2} \mathrm{P}\left(\mathrm{CH}_{2}\right)_{2} \mathrm{PPh}_{2}\right\}\right]$, again with a pyramidal $\mathrm{PCl}_{2}$ core linked into chains via chlorine bridges and with the diphosphine cross-linking the chains (Fig. 56) [309]. The only reported diarsine complex is different again and like the $\mathrm{Ge}(\mathrm{II})$ analogue is cationic, [ $\left.\mathrm{SnCl}\left\{0-\mathrm{C}_{6} \mathrm{H}_{4}\left(\mathrm{AsMe}_{2}\right)_{2}\right\}\right]\left[\mathrm{SnCl}_{3}\right]$. The diphosphine complexes have modest solubilities in chlorocarbon solvents, and the ${ }^{31} \mathrm{P}\left\{{ }^{1} \mathrm{H}\right\}$ NMR spectrum of $\left[\mathrm{SnCl}_{2}\left\{0-\mathrm{C}_{6} \mathrm{H}_{4}\left(\mathrm{PMe}_{2}\right)_{2}\right\}\right]$ showed clearly resolved ${ }^{117 / 119} \mathrm{Sn}$ satellites at ambient temperatures, while for $\left[\mathrm{SnCl}_{2}\left\{\mathrm{Me}_{2} \mathrm{P}\left(\mathrm{CH}_{2}\right)_{2} \mathrm{PMe}_{2}\right\}\right]$ some cooling of the solution was necessary to resolve the satellites. The other complexes seemed to be exchanging in solution even at low temperatures, and a combination of exchange processes and poor solubility prevented observation of ${ }^{119} \mathrm{Sn}$ resonances for any of these compounds [309].

In situ ${ }^{31} \mathrm{P}$ and ${ }^{119} \mathrm{Sn}$ NMR data have been collected from $\mathrm{MeNO}_{2}$ solutions of $\mathrm{Sn}\left(\mathrm{SbF}_{6}\right)_{2}$ and various polydentate phosphines including $\mathrm{Ph}_{2} \mathrm{P}\left(\mathrm{CH}_{2}\right)_{2} \mathrm{PPh}_{2}, \quad \mathrm{PhP}\left(\mathrm{CH}_{2} \mathrm{CH}_{2} \mathrm{PPh}_{2}\right)_{2}$, $\mathrm{MeC}\left(\mathrm{CH}_{2} \mathrm{PPh}_{2}\right)_{3},\left\{\mathrm{Ph}_{2} \mathrm{PCH}_{2} \mathrm{CH}_{2} \mathrm{P}(\mathrm{Ph}) \mathrm{CH}_{2}\right\}_{2}$ and $\mathrm{P}\left(\mathrm{CH}_{2} \mathrm{CH}_{2} \mathrm{PPh}_{2}\right)_{3}$ $[311,312]$. Although the data mostly indicated that three phosphine donors are bound to the tin, the interpretation is not unequivocal 


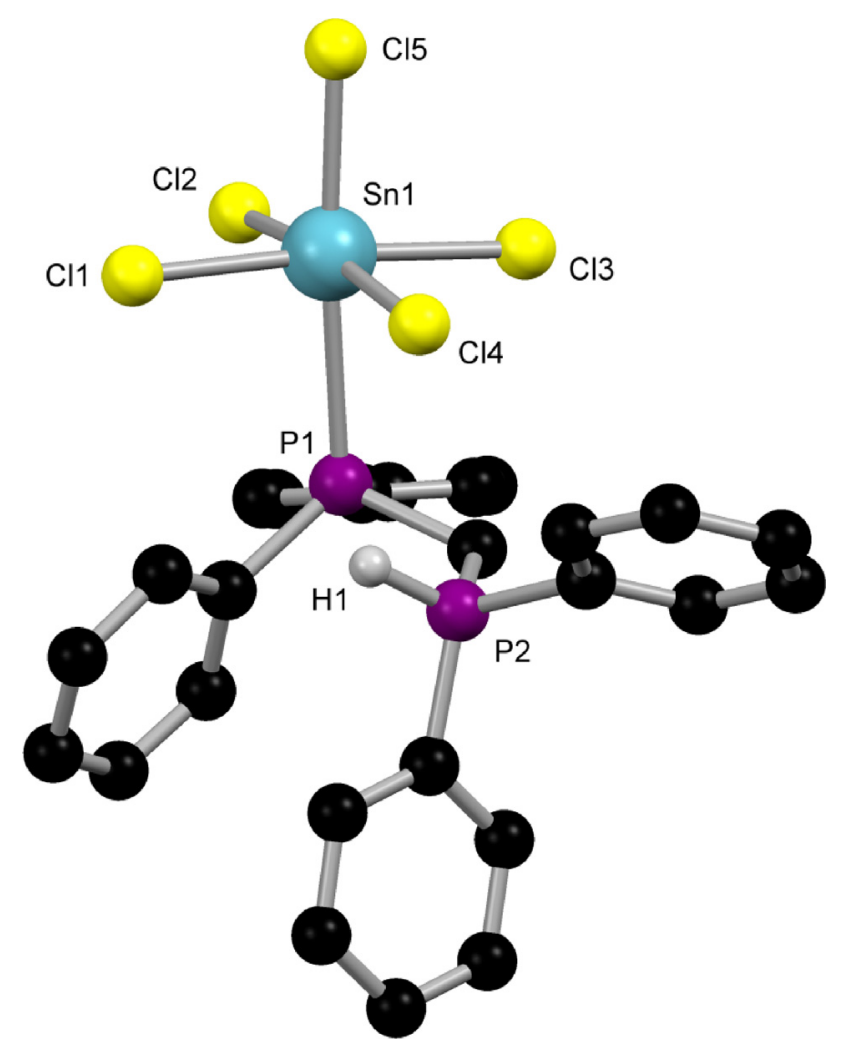

Fig. 52. View of the structure of the zwitterion [ $\left.\mathrm{SnCl}_{5}\left\{\mathrm{Ph}_{2} \mathrm{P}\left(\mathrm{CH}_{2}\right)_{2} \mathrm{P}(\mathrm{H}) \mathrm{Ph}_{2}\right\}\right]$ redrawn from Ref. [288].

and none of the complexes have been isolated. Curiously, the $\mathrm{P}, \mathrm{N}$-bidentate, $\mathrm{Ph}_{2} \mathrm{PCH}_{2}\left(2-\mathrm{C}_{5} \mathrm{H}_{4} \mathrm{~N}\right)$, fails to form a complex with $\mathrm{SnCl}_{2}$ directly, but the lithium salt $\left[\mathrm{Ph}_{2} \mathrm{PCH}\left(2-\mathrm{C}_{5} \mathrm{H}_{4} \mathrm{~N}\right)\right]^{-} \mathrm{Li}^{+}$reacts with $\mathrm{SnCl}_{2}$ in the to form $\left[\mathrm{SnCl}_{2}\left\{\mathrm{Ph}_{2} \mathrm{PCH}_{2}\left(2-\mathrm{C}_{5} \mathrm{H}_{4} \mathrm{~N}\right)\right\}\right]$, having become re-protonated in the reaction. Crystals obtained from this reaction were identified as $\left[\mathrm{SnCl}\left\{\mathrm{Ph}_{2} \mathrm{PCH}_{2}\left(2-\mathrm{C}_{5} \mathrm{H}_{4} \mathrm{~N}\right)\right\}\right]\left[\mathrm{SnCl}_{3}\right]$ with $\mathrm{P}, \mathrm{N}$-coordination, but in solution the phosphine donor easily dissociates, with complex temperature dependent equilibria present [313].

Phosphino-groups are also present in complexes of $\mathrm{Sn}$ (II) or $\mathrm{Sn}$ (IV) with anionic ligands, where the charge is on $\mathrm{O}, \mathrm{N}$ or S. Examples of $\mathrm{Sn}(\mathrm{II})$ include $\left.\left[\mathrm{Sn}_{\{}{ }^{t} \mathrm{Bu}_{2} \mathrm{PCH}_{2} \mathrm{C}\left(\mathrm{CF}_{3}\right)_{2} \mathrm{O}\right\}_{2}\right]$ which is similar to the germanium analogue discussed above [279], and
$\left[\mathrm{Sn}\left\{\mathrm{PhP}\left(\mathrm{CH}_{2}\right)_{2} \mathrm{~S}\right\}_{2}\right]$ which has a trigonal $\mathrm{S}_{2} \mathrm{P}$ core with longer thiolate bridges linking the molecules into spiral chains [314]. Other examples are $\mathrm{Sn}(\mathrm{II})$ and $\mathrm{Sn}(\mathrm{IV})$ complexes of phosphinothiolates [315-317] and the PNP pincer $\mathrm{N}\left\{\mathrm{C}_{6} \mathrm{H}_{4}(4-\mathrm{Me}) \mathrm{P}^{i} \mathrm{Pr}_{2}\right\}_{2}$ [318], and $\mathrm{Sn}(\mathrm{IV})$ complexes of bis(phenolate)phosphines [319].

\subsection{Lead}

Although the first lead dihalide complex of a diphosphine was reported in 1960 [7], there have been very few further attempts to make $\mathrm{Pb}-\mathrm{PR}_{3}$ bonds. The only recent examples are $\left[\left(\mathrm{PR}_{3}\right)_{2} \mathrm{~Pb}\left\{\mathrm{Cr}(\mathrm{CO})_{5}\right\}_{2}\right] \quad\left(\mathrm{R}=\mathrm{Me}\right.$, Et or $\left.{ }^{n} \mathrm{Bu}\right)$ which contain fourcoordinate lead [320]. All three were structurally characterised with the $\mathrm{Pb}-\mathrm{P}$ bond increasing in length from $2.76 \AA(\mathrm{R}=\mathrm{Me})$ to $2.85 \AA\left(\mathrm{R}={ }^{n} \mathrm{Bu}\right)$. In situ ${ }^{31} \mathrm{P}\left\{{ }^{1} \mathrm{H}\right\}$ and ${ }^{207} \mathrm{~Pb}$ NMR studies of $\mathrm{Pb}\left(\mathrm{SbF}_{6}\right)_{2}$ with various polydentate phosphines $\left(\mathrm{Ph}_{2} \mathrm{P}\left(\mathrm{CH}_{2}\right)_{2} \mathrm{PPh}_{2}\right.$, $\mathrm{PhP}\left(\mathrm{CH}_{2} \mathrm{CH}_{2} \mathrm{PPh}_{2}\right)_{2}, \mathrm{MeC}\left(\mathrm{CH}_{2} \mathrm{PPh}_{2}\right)_{3},\left(\mathrm{Ph}_{2} \mathrm{PCH}_{2} \mathrm{CH}_{2} \mathrm{P}(\mathrm{Ph}) \mathrm{CH}_{2}\right)_{2}$ and $\left.\mathrm{P}\left(\mathrm{CH}_{2} \mathrm{CH}_{2} \mathrm{PPh}_{2}\right)_{3}\right)$ and corresponding phosphine oxides in $\mathrm{MeNO}_{2}$ solution have been reported, although no complexes were isolated [311,312]. Usually ${ }^{31} \mathrm{P} \_{ }^{207} \mathrm{~Pb}$ couplings were resolved at ambient temperatures, and the data suggest that $1: 1$ ligand: $\mathrm{Pb}$ complexes are the major species present. Clearly, isolation of these complexes and structural characterisation in the solid state is desirable. Lead(II)phosphine bonds are also present in the phosphinothiolate complexes $\left[\mathrm{Pb}\left(2-\mathrm{Ph}_{2} \mathrm{PC}_{6} \mathrm{H}_{4} \mathrm{~S}\right)_{2}\right]$ and $\left[\mathrm{Pb}\left(2-\mathrm{Ph}_{2} \mathrm{P}-6-\mathrm{Me}_{3} \mathrm{Si}_{-} \mathrm{C}_{6} \mathrm{H}_{3} \mathrm{~S}\right)_{2}\right]$, obtained by anodic dissolution of lead in an $\mathrm{MeCN}$ solution of the ligand [321].

\section{Group 15}

Phosphine or arsine adducts of phosphorus(III) or arsenic(III) halides have been known for many years [7] but were relatively little studied until the 1990s. More recently, much effort has been devoted to elucidating the chemistry of phosphenium $\left[\mathrm{R}_{2} \mathrm{P}\right]^{+}$and arsenium $\left[\mathrm{R}_{2} \mathrm{As}\right]^{+}$cations and their heavier Group 15 analogues. These are six-electron unsaturated species containing a lone pair but also a vacant acceptor orbital, and are stabilised by donor ligands such as phosphines, forming $\left[\mathrm{R}_{3} \mathrm{P} \rightarrow \mathrm{PR}_{2}\right]^{+},\left[\mathrm{R}_{3} \mathrm{P} \rightarrow \mathrm{AsR}_{2}\right]^{+}$, $\left[\left(R_{3} P\right)_{2} \rightarrow P_{2}\right]^{+}$etc. species. The most generally used syntheses combine the organophosphorus or organoarsenic halide with the phosphine or arsine donor and a halide abstractor such as $\mathrm{AlCl}_{3}$ or $\mathrm{GaCl}_{3}$, although the latter is sometimes replaced by a large weakly coordinating anion such as $\left[\mathrm{PF}_{6}\right]^{-}$. Although they formally fall within the scope of the present article, these complexes also

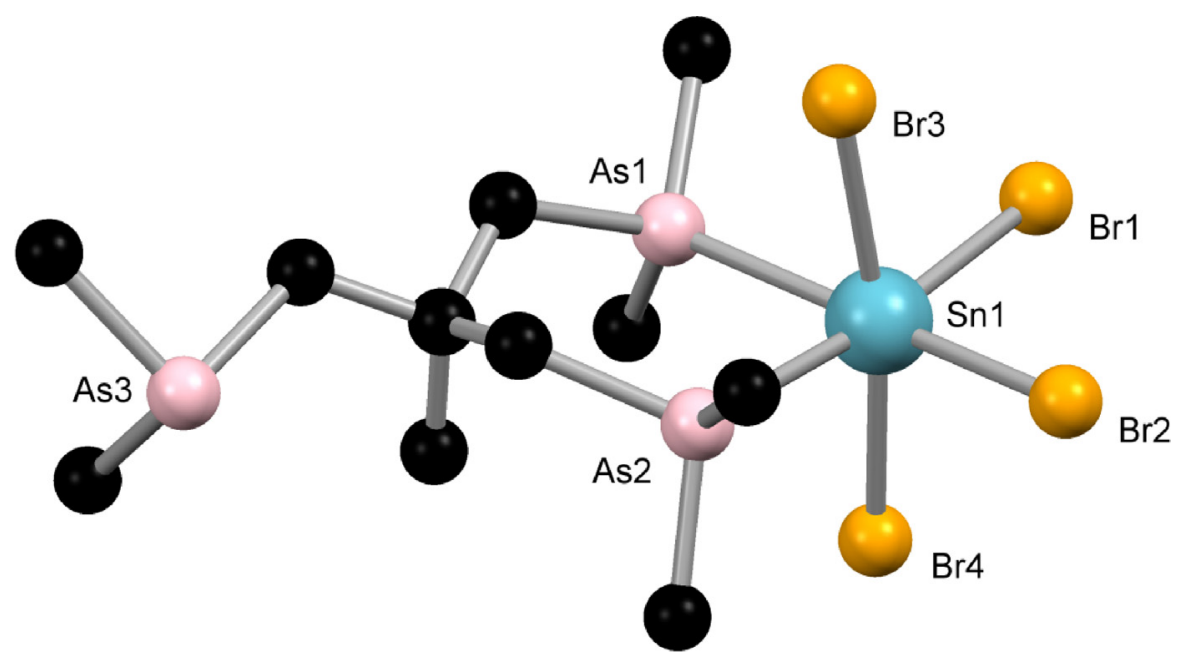

Fig. 53. View of the structure of $\left[\mathrm{SnBr}_{4}\left\{\mathrm{MeC}\left(\mathrm{CH}_{2} \mathrm{AsMe}_{2}\right)_{3}\right\}\right]$ redrawn from Ref. [281]. 


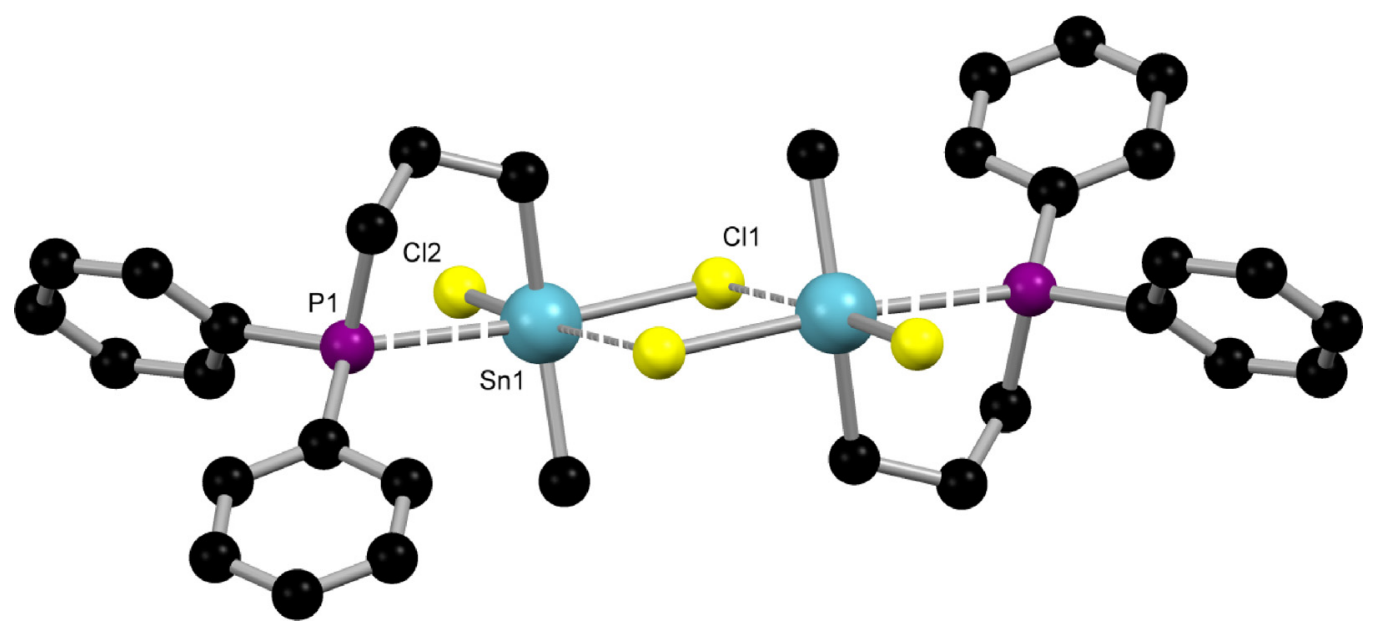

Fig. 54. View of the structure of $\left[\left\{\mathrm{SnCl}_{2} \mathrm{Me}\left\{\mathrm{Ph}_{2} \mathrm{P}\left(\mathrm{CH}_{2}\right)_{3}\right\}\right\}_{2}\right]$ redrawn from Ref. [307].

link to the extensive chemistry of Group 15 compounds with P-P, $\mathrm{P}-\mathrm{As}$ bonds etc. and have been discussed in recent review articles [322,323]. We have therefore (somewhat arbitrarily) limited coverage in this article to the discussion of "classical" neutral adducts of Group 15 trihalides with pnictogen ligands to provide a comparison with the chemistries discussed in previous sections. In some cases the bonding in the latter compounds has been viewed as intermediate between covalent $\left[\mathrm{R}_{3} \mathrm{P}-\mathrm{PX}_{3}\right]$ and ionic $\left[\mathrm{R}_{3} \mathrm{P}-\mathrm{PX}_{2}\right] \mathrm{X}$, which links them (at least formally) to phosphenium salts $\left[\mathrm{R}_{3} \mathrm{P}-\mathrm{PR}_{2}\right]^{+}$. The $\mathrm{EX}_{3}(\mathrm{E}=\mathrm{As}, \mathrm{Sb}, \mathrm{Bi} ; \mathrm{X}=\mathrm{Cl}, \mathrm{Br}$ or $\mathrm{I}$-there are no examples with F) are modest Lewis acids which form rather weak bonds to $E_{3}$ donors, whilst the pentahalides all seem to halogenate $\mathrm{ER}_{3}$. The isolated complexes are labile in solution which means that solution NMR spectroscopic data are usually uninformative, whilst even in the solid state the distorted structures are not easily deduced by vibrational spectroscopy. Hence X-ray crystallography is the key technique in this group.

\subsection{Phosphorus}

Apart from the work on phosphenium compounds referred to above, there has been little new work on phosphorus halide complexes. The structure of the $1: 1$ adduct between $\mathrm{PBr}_{3}$ and $\mathrm{PMe}_{3}$ consists of two square pyramidal units linked into a dimer by very asymmetric bromine bridges $\left[\left(\mathrm{PMe}_{3}\right) \mathrm{PBr}_{2}(\mu-\mathrm{Br})_{2} \mathrm{PBr}_{2}\left(\mathrm{PMe}_{3}\right)\right]$ with $\mathrm{P}-\mathrm{Br}_{\text {terminal }}=2.424(2), 2.250(2) \AA$ and $\mathrm{P}-\mathrm{Br}_{\text {bridge }}=2.677(2)$, 3.327(2) А [324].

\subsection{Arsenic}

$\mathrm{PMe}_{3}$ forms 2:1 complexes with $\mathrm{AsX}_{3}(\mathrm{X}=\mathrm{Cl}, \mathrm{Br}$ or I), but only with $\mathrm{AsCl}_{3}$ can a 1:1 complex also be isolated [325]. The structure of the latter reveals a dimeric core with square-pyramidal units arranged anti (Fig. 57) and long As $-\mathrm{Cl}$ contacts ( $\sim 3.43 \AA$ ) linking the units into infinite chains. The solution $\left(\mathrm{CDCl}_{3}\right)^{1} \mathrm{H}$ NMR spectra of either $\left[\mathrm{AsCl}_{3}\left(\mathrm{PMe}_{3}\right)_{\mathrm{x}}\right](x=1$ or 2$)$ show two doublets, indicating both species are present in solution, and the ${ }^{31} \mathrm{P}\left\{{ }^{1} \mathrm{H}\right\}$ NMR spectra show only a single broad resonance indicative of fast exchange. Both $\mathrm{AsMe}_{3}$ and $\mathrm{AsEt}_{3}$ form only 1:1 adducts with $\mathrm{AsCl}_{3}$, which are dimeric, but without any evidence for longer contacts [325,326]. Diphosphines or diarsines also form $1: 1$ adducts with arsenic(III) halides, including [As $\mathrm{X}_{3}\{0-$ $\left.\left.\mathrm{C}_{6} \mathrm{H}_{4}\left(\mathrm{AsMe}_{2}\right)_{2}\right\}\right](\mathrm{X}=\mathrm{Cl}, \mathrm{Br}$ or I $)$ and $\left[\mathrm{AsCl}_{3}\left\{0-\mathrm{C}_{6} \mathrm{H}_{4}\left(\mathrm{PR}_{2}\right)_{2}\right\}\right](\mathrm{R}=\mathrm{Ph}$ or Me) [325]. The $\left[\mathrm{AsX}_{3}\left\{0-\mathrm{C}_{6} \mathrm{H}_{4}\left(\mathrm{AsMe}_{2}\right)_{2}\right\}\right]$ ( $\mathrm{X}=\mathrm{Br}$ or I) have dimeric structures based upon edge-shared bi-octahedra (Fig. 58),

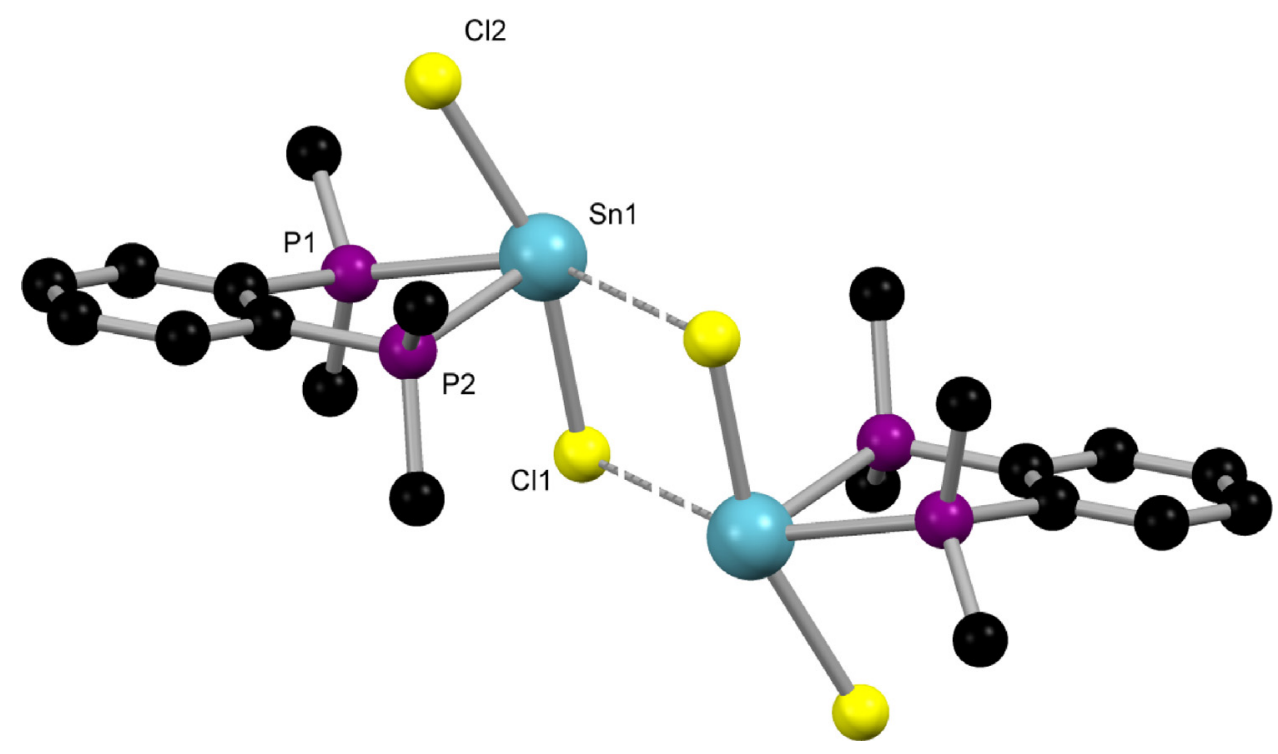

Fig. 55. View of the structure of $\left[\left\{\mathrm{SnCl}_{2}\left\{0-\mathrm{C}_{6} \mathrm{H}_{4}\left(\mathrm{PMe}_{2}\right)_{2}\right\}\right\}_{2}\right]$ redrawn from Ref. [309]. 


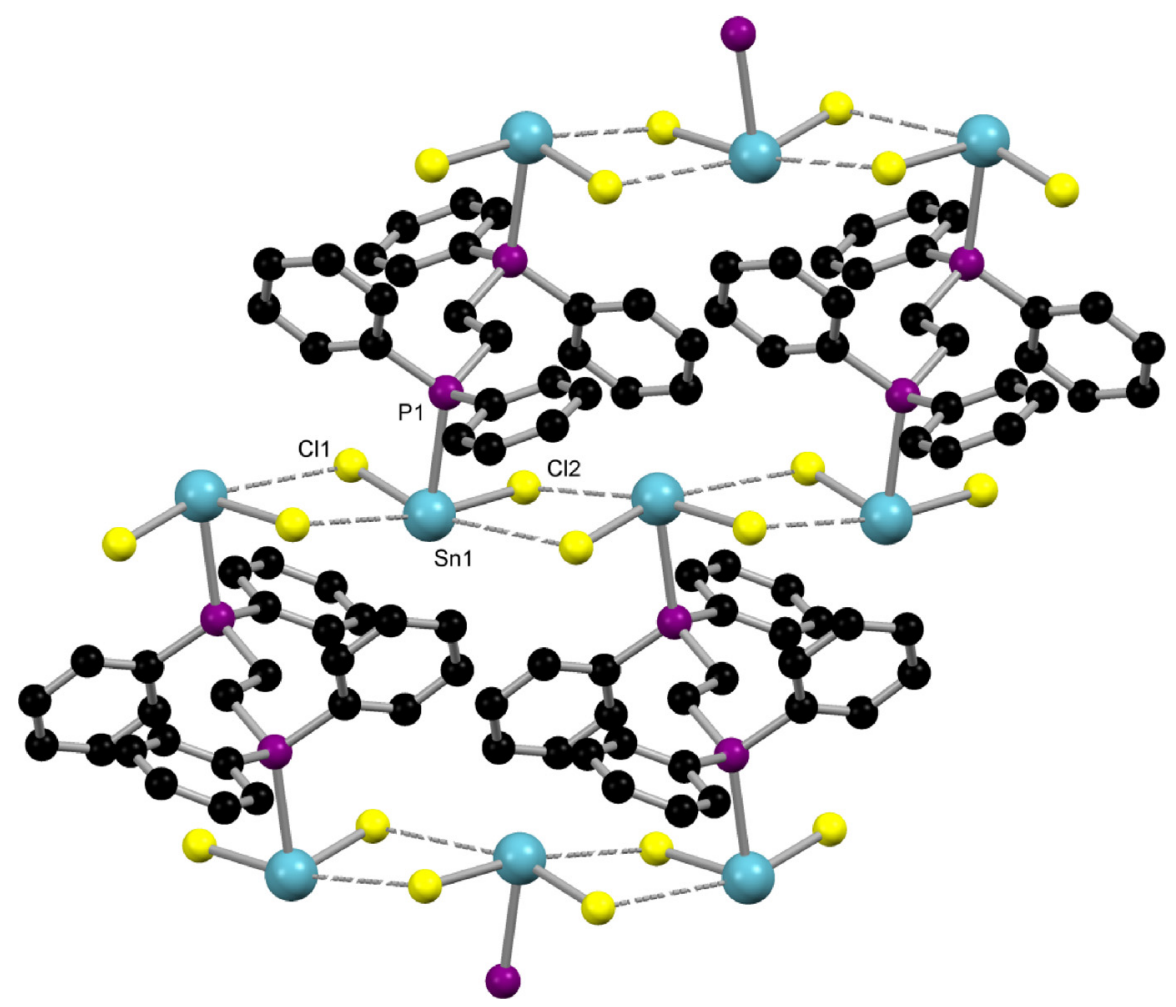

Fig. 56. View of the polymeric structure of $\left[\left(\mathrm{SnCl}_{2}\right)_{2}\left\{\mu-\mathrm{Ph}_{2} \mathrm{P}\left(\mathrm{CH}_{2}\right)_{2} \mathrm{PPh}_{2}\right\}\right]$ redrawn from Ref. [309].

with distorted environments about the central arsenics; there is no vacant vertex which could be occupied by the arsenic (on $\mathrm{AsX}_{3}$ ) lone pair, but distortions in the bond lengths and angles suggest the lone pair may be localised in the direction of one $\mathrm{AsX}_{3}$ triangular face [325]. The $\left[\mathrm{AsCl}_{3}\left\{0-\mathrm{C}_{6} \mathrm{H}_{4}\left(\mathrm{PPh}_{2}\right)_{2}\right\}\right]$ decomposes in $\mathrm{CDCl}_{3}$ solution to give a mixture of species including the chlorinated diphosphine (based on ${ }^{31} \mathrm{P}\left\{{ }^{1} \mathrm{H}\right\}$ NMR evidence). Arsenic(III) iodide is reduced by $\mathrm{Ph}_{2} \mathrm{P}\left(\mathrm{CH}_{2}\right)_{2} \mathrm{PPh}_{2}$ to the arsenium cation $\left[\mathrm{As}\left\{\mathrm{Ph}_{2} \mathrm{P}\left(\mathrm{CH}_{2}\right)_{2} \mathrm{PPh}_{2}\right\}\right] \mathrm{I}$ and the cation has been structurally authenticated in $\left[\mathrm{As}\left\{\mathrm{Ph}_{2} \mathrm{P}\left(\mathrm{CH}_{2}\right)_{2} \mathrm{PPh}_{2}\right\}\right]\left[\mathrm{As}_{2} \mathrm{I}_{7}\left\{\mathrm{Ph}_{2} \mathrm{P}\left(\mathrm{CH}_{2}\right)_{2} \mathrm{PPh}_{2}\right\}\right]$ $[327,328]$. The triarsine $\mathrm{MeC}\left(\mathrm{CH}_{2} \mathrm{AsMe}_{2}\right)_{3}$ forms 1:1 adducts with $\mathrm{AsX}_{3}$ of unknown structure [325].

\subsection{Antimony}

Complexes of antimony(III) halides are mostly six-coordinate, with distorted octahedral antimony. An exception is the dimeric $\left[\mathrm{Sb}_{2} \mathrm{I}_{4}(\mu-\mathrm{I})_{2}\left(\mathrm{PMe}_{3}\right)_{2}\right]$.thf which has five-coordinate antimony

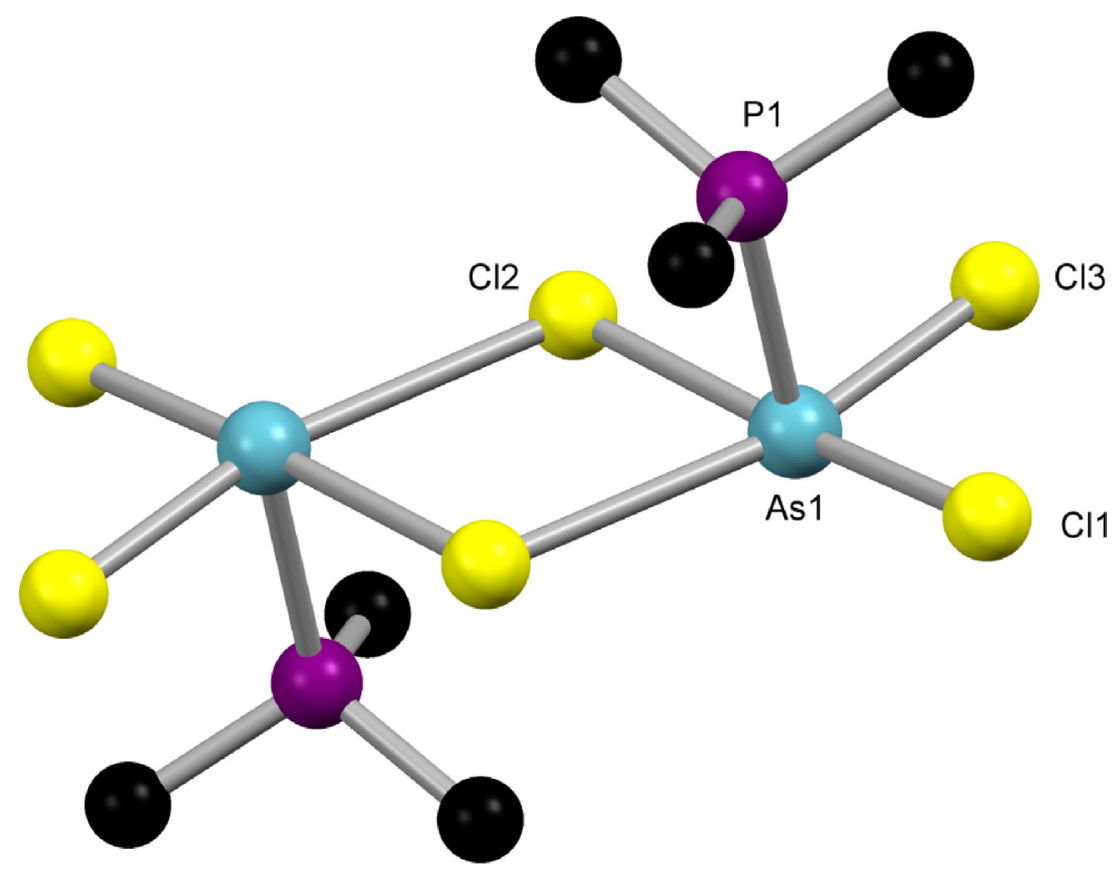

Fig. 57. View of the structure of $\left[\mathrm{As}_{2} \mathrm{Cl}_{4}(\mu-\mathrm{Cl})_{2}\left(\mathrm{PMe}_{3}\right)_{2}\right]$ redrawn from Ref. [325]. 


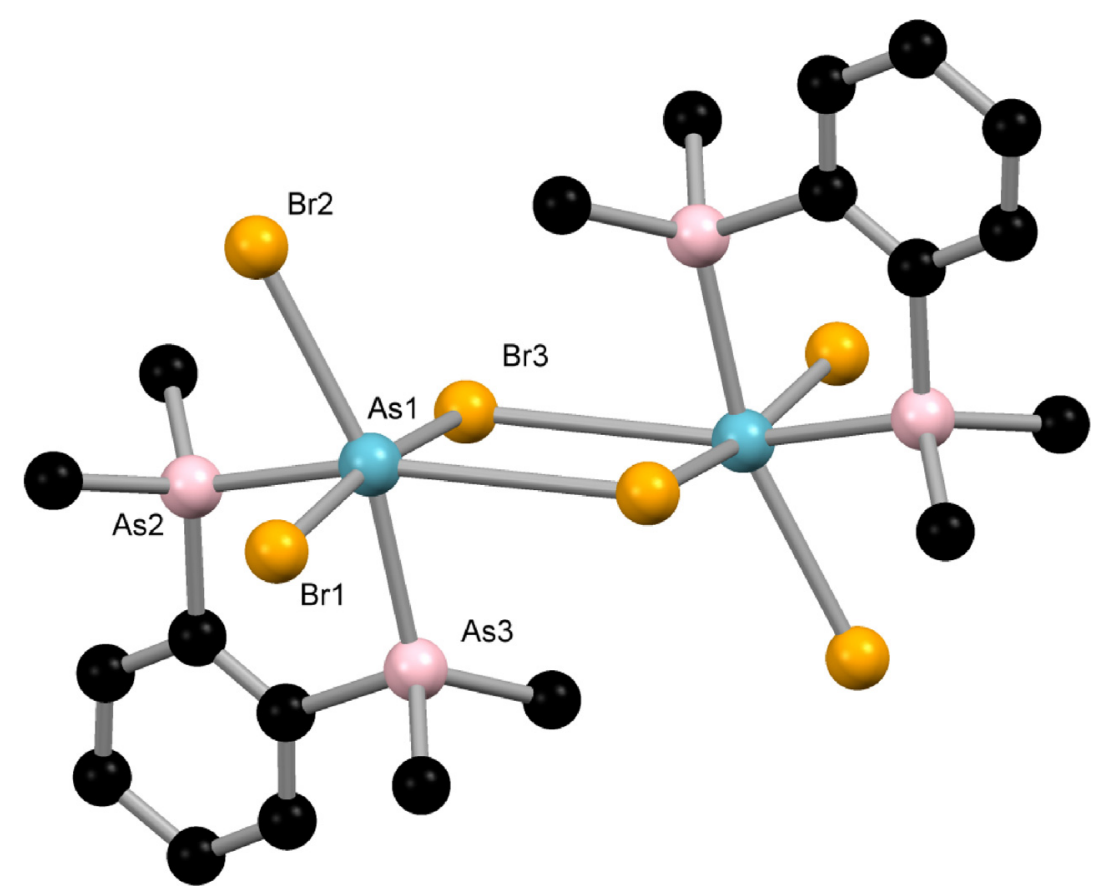

Fig. 58. View of the structure of $\left[\mathrm{As}_{2} \mathrm{Br}_{4}(\mu-\mathrm{Br})_{2}\left\{0-\mathrm{C}_{6} \mathrm{H}_{4}\left(\mathrm{AsMe}_{2}\right)_{2}\right\}_{2}\right]$ redrawn from Ref. [325].

centres with anti-disposed $\mathrm{PMe}_{3}$ groups axial, weakly associated into polymer chains via long Sb-I contacts [329]. In [ $\left.\mathrm{SbCl}_{3}\left(\mathrm{AsEt}_{3}\right)\right]$ the antimony has a disphenoidal shape with equatorial $\mathrm{AsEt}_{3}$ (alternatively described as trigonal bipyramidal with a vacant equatorial vertex) with these units linked via long $\mathrm{Sb}-\mathrm{Cl}$ bonds into zig-zag chains (Fig. 59) [330]. In contrast, the yellow crystals formed from $\mathrm{SbBr}_{3}$ and $\mathrm{PEt}_{3}$ in thf solution, seemingly the product of adventitious hydrolysis, were found to be the anionic $\left[\mathrm{PEt}_{3} \mathrm{H}\right]\left[\mathrm{Sb}_{2} \mathrm{Br}_{7}\left(\mathrm{PEt}_{3}\right)_{2}\right]$ with discrete confacial bioctahedral anions (Fig. 60) [331]. The reactions of $\mathrm{SbX}_{3}$ and $\mathrm{SbR}_{3}$ typically result in scrambling to give $S_{b R_{3-n}} \mathrm{X}_{n}$, but stibine adducts of antimony halides include the structurally characterised $\left[\mathrm{SbI}_{3}\left(\mathrm{SbMe}_{3}\right)(\mathrm{thf})\right]$, which is a centrosymmetric dimer with two iodide bridges [332], and $\left[\mathrm{SbI}_{2} \mathrm{Me}\left(\mathrm{SbMe}_{3}\right)\right]$ [333], which has a trans-trigonal bipyramidal shape with a vacant equatorial vertex for the (Sb) $\mathrm{SbI}_{2}$ Me unit.

A discrete anion $\left[\mathrm{SbI}_{4}\left\{\mathrm{Me}_{2} \mathrm{P}\left(\mathrm{CH}_{2}\right)_{2} \mathrm{PMe}_{2}\right\}\right]^{-}$was formed on recrystallisation of $\left[\mathrm{SbI}_{3}\left\{\mathrm{Me}_{2} \mathrm{P}\left(\mathrm{CH}_{2}\right)_{2} \mathrm{PMe}_{2}\right\}\right]$ from pyridine, and has a distorted octahedral geometry [334]. However, the most common type of complex formed by diphosphine or diarsine ligands (there are no known distibine complexes) with $\mathrm{SbX}_{3}$ are dimeric $\left.\left[\left\{\mathrm{SbX}_{3} \text { (ligand }\right)\right\}_{2}\right]$ with unsymmetric halide bridges. The complex $\left[\mathrm{SbBr}_{3}\left\{\mathrm{Me}_{2} \mathrm{P}\left(\mathrm{CH}_{2}\right)_{2} \mathrm{PMe}_{2}\right\}\right]$ has two polymorphs, one the familiar dimer (Fig. 61(a)), the second a tetramer composed of a central dimer unit linked via single $\mathrm{Br}$ bridges to two monomer units (Fig. 61(b)) [329]. Complexes similar to the dimer are $\left[\left\{\mathrm{SbX}_{3}\left(o-\mathrm{C}_{6} \mathrm{H}_{4}\left(\mathrm{AsMe}_{2}\right)_{2}\right)\right\}_{2}\right],\left[\left\{\mathrm{SbX}_{3}\left(o-\mathrm{C}_{6} \mathrm{H}_{4}\left(\mathrm{PMe}_{2}\right)_{2}\right)\right\}_{2}\right],\left[\left\{\mathrm{SbX}_{3}(o-\right.\right.$ $\left.\left.\left.\mathrm{C}_{6} \mathrm{H}_{4}\left(\mathrm{PPh}_{2}\right)_{2}\right)\right\}_{2}\right]$ and $\left[\left\{\mathrm{SbX}_{3}\left\{\mathrm{Ph}_{2} \mathrm{As}\left(\mathrm{CH}_{2}\right)_{2} \mathrm{AsPh}_{2}\right\}\right\}_{2}\right](\mathrm{X}=\mathrm{Cl}, \mathrm{Br}$ or

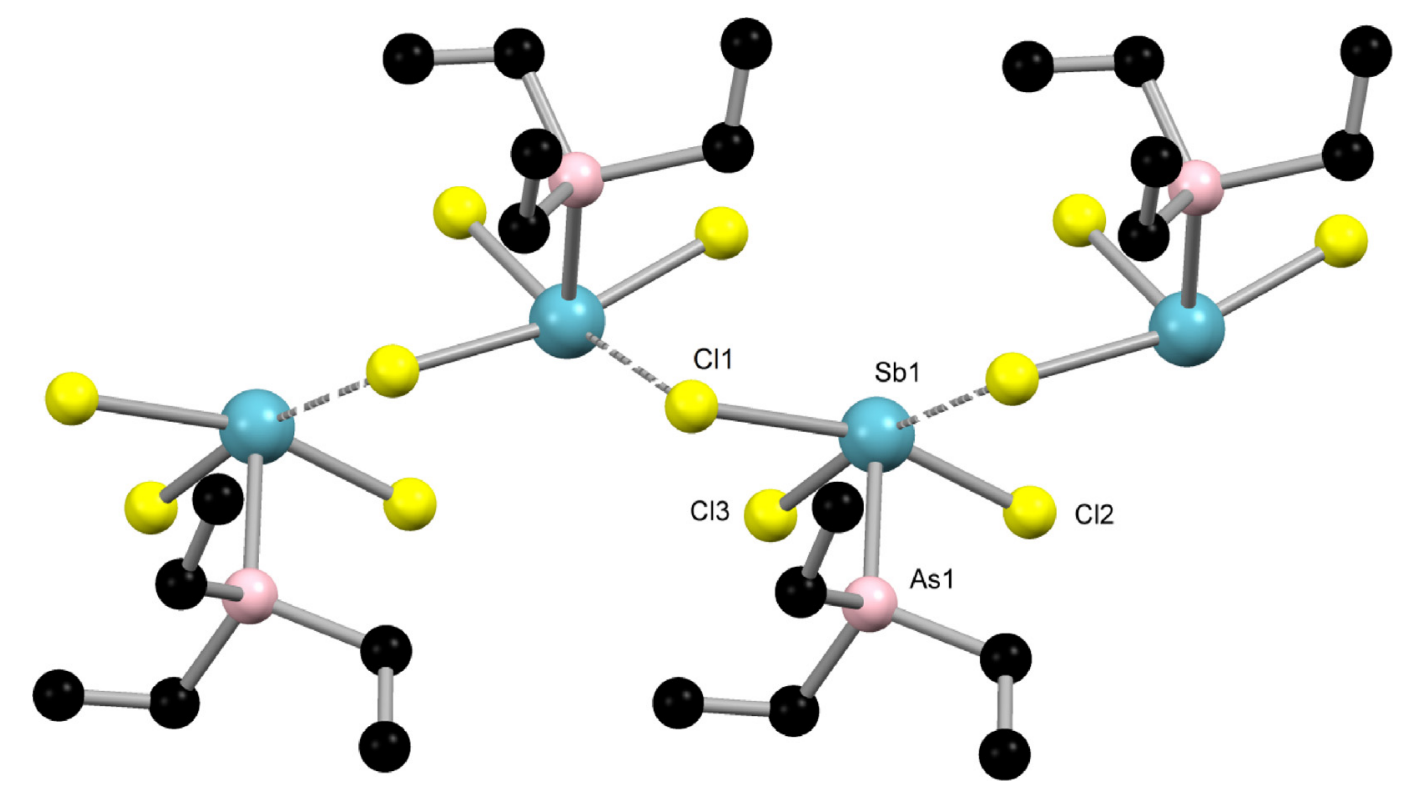

Fig. 59. View of the chain structure of $\left[\mathrm{SbCl}_{3}\left(\mathrm{AsEt}_{3}\right)\right]$ redrawn from Ref. [330]. 


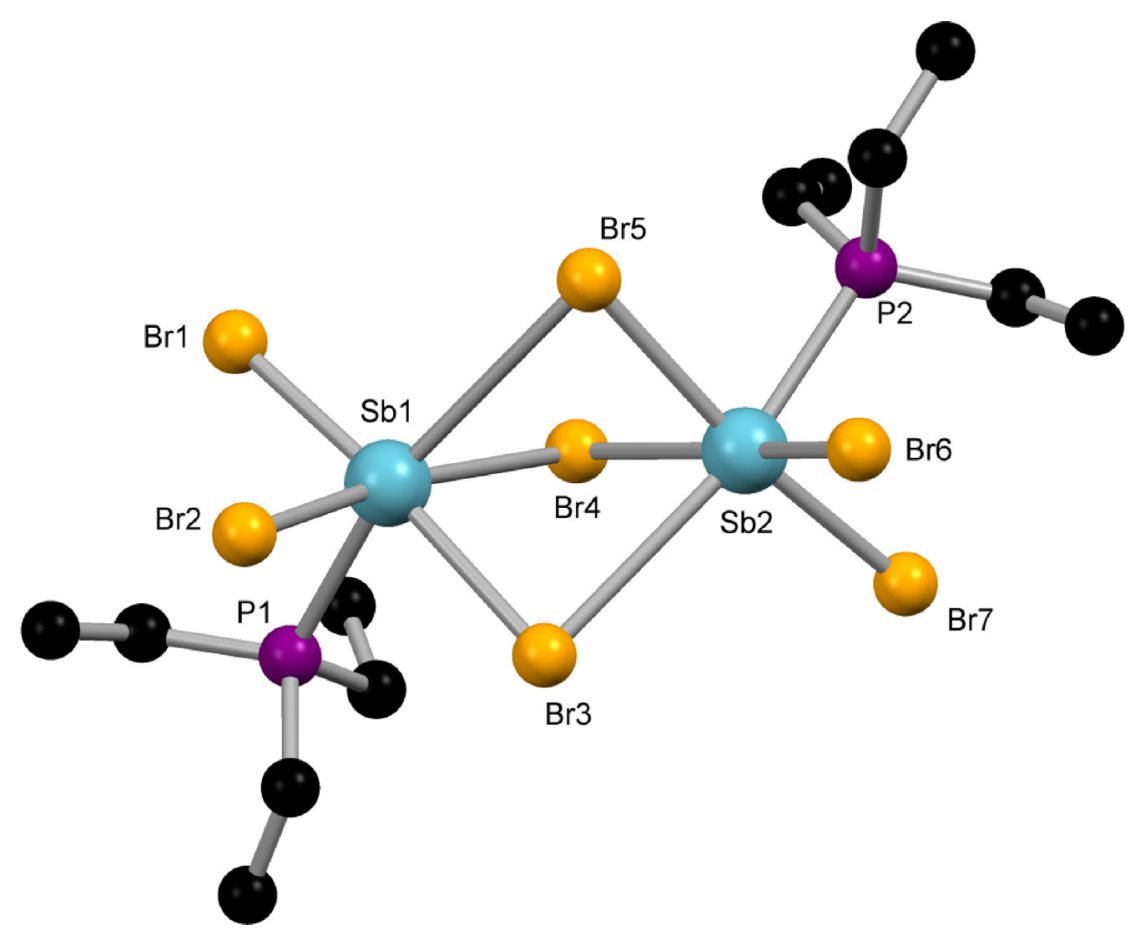

Fig. 60. View of the structure of the anion $\left[\mathrm{Sb}_{2} \mathrm{Br}_{7}\left(\mathrm{PEt}_{3}\right)_{2}\right]^{-}$redrawn from Ref. [331].

I) [335]. Recrystallisation of $\left[\left\{\mathrm{SbCl}_{3}\left(0-\mathrm{C}_{6} \mathrm{H}_{4}\left(\mathrm{AsMe}_{2}\right)_{2}\right)\right\}_{2}\right]$ from ethanol gave block crystals of $\left[\mathrm{Sb}_{2} \mathrm{Cl}_{6}\left\{0-\mathrm{C}_{6} \mathrm{H}_{4}\left(\mathrm{AsMe}_{2}\right)_{2}\right\}\right]$ which is a sheet polymer with distorted octahedral $\mathrm{SbCl}_{4} \mathrm{As}_{2}$ units linked to square pyramidal $\mathrm{SbCl}_{5}$ groups via zig-zag chains, cross linked by longer chloride bridges into sheets [335]. The structures of all the antimony halide complexes show varying degrees of distortion about the central antimony and a range of bond lengths, which seem to result from a number of factors $[8,18]$. From the bond length and angle data the dominant core unit is the pyramidal $\mathrm{SbX}_{3}$ group, linked to more weakly bound neutral donors and long halide bridges. The $3 c-4 e$ bonding model can account for the disparate bond lengths, but in addition, there seem to be varying degrees of stereochemical activity of the antimony based lone pair. In the six-coordinate complexes there is no available vertex position, but distortions of the octahedra have been rationalised as due to localisation of the lone pair along an edge or face. Since the $\mathrm{SbX}_{3}$-ligand interactions are weak, some distortions of the coordination sphere may also be caused by intermolecular packing. Solution NMR data on the complexes are consistent with weak binding of the neutral ligands, and rapid ligand exchange in solution.

\subsection{Bismuth}

The complexes of bismuth(III) halides generally follow the pattern established for antimony, although they are rather more stable and the structures less distorted. $\mathrm{BiBr}_{3}$ reacts with neat $\mathrm{PMe}_{3}$ to form $\left[\mathrm{BiBr}_{3}\left(\mathrm{PMe}_{3}\right)_{2}\right]$; crystals obtained from $\mathrm{CH}_{2} \mathrm{Cl}_{2}$ solution show that the structure is dimeric $\left[\mathrm{Br}_{2}\left(\mathrm{PMe}_{3}\right)_{2} \mathrm{Bi}(\mu-\mathrm{Br})_{2} \mathrm{BiBr}_{2}\left(\mathrm{PMe}_{3}\right)_{2}\right]$ (Fig. 62) [336]. The corresponding reaction with $\mathrm{PMe}_{2} \mathrm{Ph}$ in thf gave crystals of the mixed phosphine-phosphine oxide complex $\left[\left\{\mathrm{BiBr}_{3}\left(\mathrm{PMe}_{2} \mathrm{Ph}\right)\left(\mathrm{OPMe}_{2} \mathrm{Ph}\right)\right\}_{2}\right]$, presumably due to air-oxidation. The structure is similar to that of the $\mathrm{PMe}_{3}$ complex, with the phosphine oxides occupying axial positions on each bismuth [336]. The complexes formed in these systems are very sensitive to small changes in the reaction conditions. Thus reaction of $\mathrm{BiBr}_{3}$ and $\mathrm{PMe}_{3}$ in thf solution, followed by crystallisation of the product from $\mathrm{MeCN} / \mathrm{Et}_{2} \mathrm{O}$ gave $\left[\mathrm{PMe}_{3} \mathrm{H}\right]\left[\mathrm{Bi}_{2} \mathrm{Br}_{7}\left(\mathrm{PMe}_{3}\right)_{2}\right.$ ] [337]. The anion has a planar $\mathrm{Bi}_{2} \mathrm{Br}_{6}$ core with one $\mathrm{PMe}_{3}$ group on each bismuth arranged anti, the anions being assembled into zig-zag chains by single bromide bridges trans to $\mathrm{PMe}_{3}$ (Fig. 63). The iodide complex $\left[\mathrm{PMe}_{3} \mathrm{H}\right]\left[\mathrm{Bi}_{2} \mathrm{I}_{7}\left(\mathrm{PMe}_{3}\right)_{2}\right]$ (although not structurally characterised) appears to be similar, but the corresponding reaction between $\mathrm{BiI}_{3}$ and $\mathrm{PEt}_{3}$ in thf solution gave crystals of $\left[\mathrm{PEt}_{4}\right]_{4}\left[\mathrm{Bi}_{6} \mathrm{I}_{22}\right]$ [337]. It is unclear how the $\left[\mathrm{PEt}_{4}\right]^{+}$cation is formed.

The product from $\mathrm{BiBr}_{3}$ and $\mathrm{PEt}_{3}$ in thf is different again, forming a cubane tetramer $\left[\mathrm{Bi}_{4} \mathrm{Br}_{12}\left(\mathrm{PEt}_{3}\right)_{4}\right]$ (Fig. 64) [329] which is clearly related to the $\left[\mathrm{Bi}_{4} \mathrm{Br}_{16}\right]^{4-}$ anion, with one terminal bromide on each bismuth replaced by a phosphine. The geometry about the bismuth centres is close to octahedral and the $\mathrm{Bi}-\mathrm{Br}$ and $\mathrm{Bi}-\mathrm{P}$ bond lengths are unexceptional, apart from $\mathrm{Bi}-\mathrm{Br}_{\text {trans } \mathrm{P}}$ which are significantly longer $(\sim 0.2 \AA)$ than $\mathrm{Bi}-\mathrm{Br}_{\text {transBr }}$.

A mononuclear cis-octahedral anion is present in $\left[\mathrm{PPh}_{4}\right]\left[\mathrm{BiI}_{4}\left(\mathrm{PMe}_{2} \mathrm{Ph}\right)_{2}\right]$, formed from $\left[\mathrm{PPh}_{4}\right] \mathrm{I}, \mathrm{BiI}_{3}$ and $\mathrm{PMe}_{2} \mathrm{Ph}$ in $\mathrm{MeNO}_{2}$ solution [331]. The $\mathrm{d}(\mathrm{Bi}-\mathrm{I})$ are little different whether cis or trans to $\mathrm{P}$ (with an average length of $3.048 \AA$ ), but $\mathrm{d}(\mathrm{Bi}-\mathrm{P})$ are long at 2.981(2) and 3.005(2) $\AA$, and there are significant angle distortions-the axial $<\mathrm{I}-\mathrm{Bi}-\mathrm{I}$ is $157.1^{\circ}$ (bent towards the phosphines) and the $<\mathrm{P}-\mathrm{Bi}-\mathrm{P}$ is $112.3^{\circ}$, which was discussed in terms of lone-pair activity [331].

Diphosphines and diarsines also form complexes with $\mathrm{BiX}_{3}$, and rather surprisingly some of the former complexes oxidise very readily to phosphine oxides. The common stoichiometry is $1: 1$ and recently reported complexes are $\left[\left\{\mathrm{BiCl}_{3}\left(\mathrm{Ph}_{2} \mathrm{PCH}_{2} \mathrm{PPh}_{2}\right)\right\}_{2}\right] \quad[339], \quad\left[\left\{\mathrm{BiX}_{3}\left(\mathrm{Ph}_{2} \mathrm{P}\left(\mathrm{CH}_{2}\right)_{2} \mathrm{PPh}_{2}\right)_{2}\right\}_{2}\right]$ $\left(\mathrm{X}=\mathrm{Cl}, \quad \mathrm{Br}\right.$ or I) $[338,339], \quad\left[\mathrm{Bi}_{2} \mathrm{Cl}_{6}\left(\mathrm{Ph}_{2} \mathrm{P}\left(\mathrm{CH}_{2}\right)_{2} \mathrm{PPh}_{2}\right)_{3}\right]$ $[338,339], \quad\left[\left\{\mathrm{BiBr}_{3}\left(\mathrm{Me}_{2} \mathrm{P}\left(\mathrm{CH}_{2}\right)_{2} \mathrm{PMe}_{2}\right)\right\}_{2}\right] \quad[329], \quad\left[\left\{\mathrm{BiX}_{3}(0-\right.\right.$ $\left.\left.\left.\mathrm{C}_{6} \mathrm{H}_{4}\left(\mathrm{PPh}_{2}\right)_{2}\right)\right\}_{2}\right] \quad[335], \quad\left[\left\{\mathrm{BiX}_{3}\left(\mathrm{o}-\mathrm{C}_{6} \mathrm{H}_{4}\left(\mathrm{PMe}_{2}\right)_{2}\right)\right\}_{2}\right]$ [335], $\left[\left\{\mathrm{BiX}_{3}\left(\mathrm{o}-\mathrm{C}_{6} \mathrm{H}_{4}\left(\mathrm{AsMe}_{2}\right)_{2}\right)\right\}_{2}\right]$ [335], $\left[\left\{\mathrm{BiX}_{3}\left(\mathrm{Ph}_{2} \mathrm{As}\left(\mathrm{CH}_{2}\right)_{2} \mathrm{AsPh}_{2}\right)\right\}_{2}\right]$ [335], and $\left[\mathrm{BiX}_{3}\left\{\mathrm{MeC}\left(\mathrm{CH}_{2} \mathrm{AsMe}_{2}\right)_{3}\right\}\right](\mathrm{X}=\mathrm{Cl}, \mathrm{Br}$ or I) [335]. Similar to the antimony analogues, spectroscopic techniques yield little useful data, and do not identify the structural units present with any certainty. The complexes range in colour from pale yellow $(\mathrm{X}=\mathrm{Cl})$ through to deep red or orange $(\mathrm{X}=\mathrm{I})$, but the UV/visible spectra consist of ill-defined broad charge transfer bands in the near UV which tail into the visible, and could not be interpreted in detail [335]. The complexes are poorly soluble in weakly 

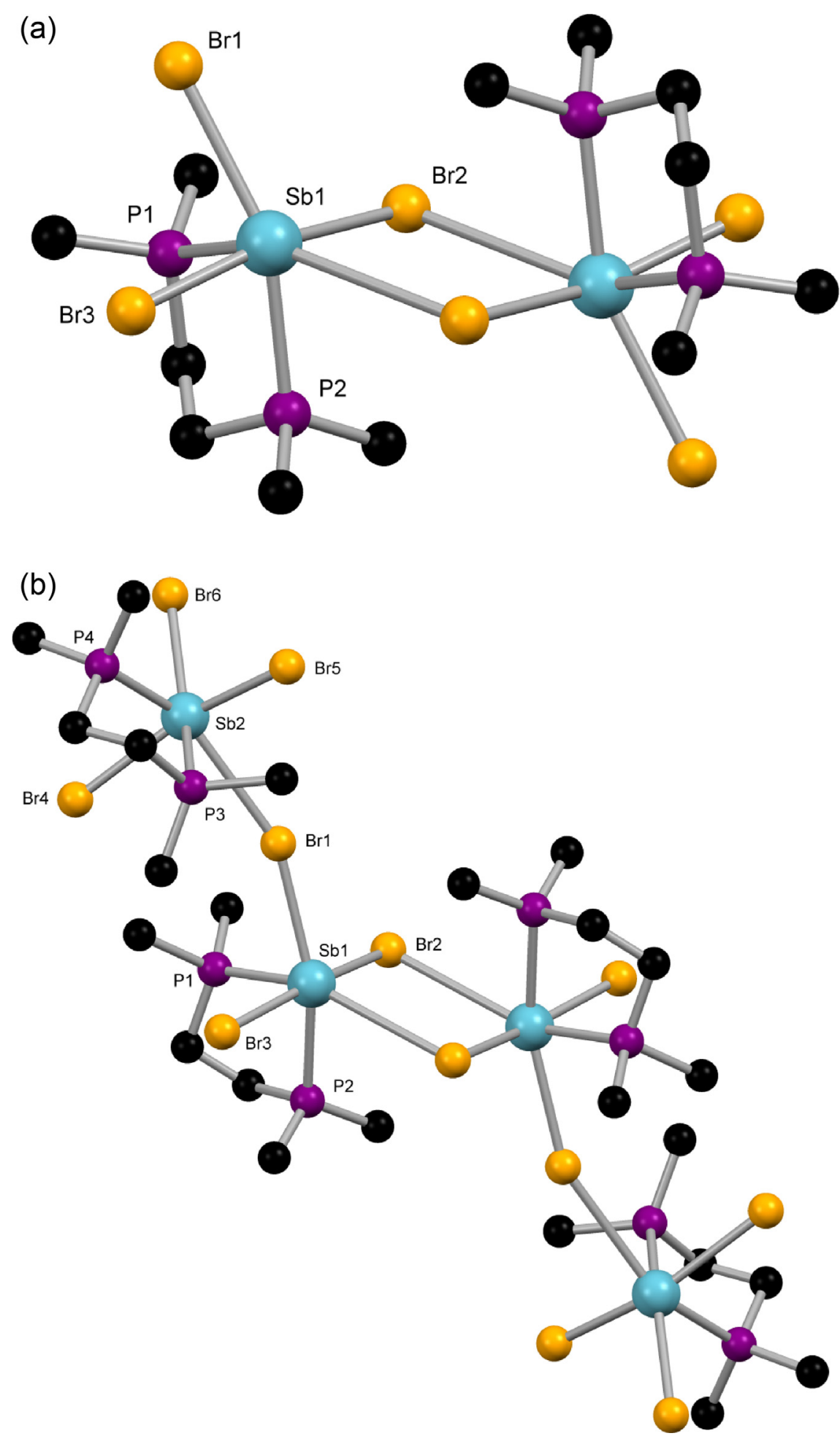

Fig. 61. (a) View of the structure of $\left[\mathrm{Sb}_{2} \mathrm{Br}_{4}(\mu-\mathrm{Br})_{2}\left\{\mathrm{Me}_{2} \mathrm{P}\left(\mathrm{CH}_{2}\right)_{2} \mathrm{PMe}_{2}\right\}_{2}\right]$ redrawn from Ref. [329]. (b) View of the structure of $\left[\left\{\mathrm{SbBr}_{3}\left\{\mathrm{Me}_{2} \mathrm{P}\left(\mathrm{CH}_{2}\right)_{2} \mathrm{PMe}_{2}\right\}\right\}_{4}\right]$ redrawn from Ref. [329].

coordinating solvents (and the phosphines are likely to be displaced by strong donor solvents), and ${ }^{1} \mathrm{H}$ or ${ }^{31} \mathrm{P}\left\{{ }^{1} \mathrm{H}\right\}$ NMR spectroscopy usually shows only small coordination shifts and, in the presence of added ligand, averaged resonances due to fast exchange. The far IR spectra show rather broad $v(\mathrm{M}-\mathrm{X})$ vibrations, again insufficient data to provide reliable structural information, although for the phosphine complexes both IR and ${ }^{31} \mathrm{P}\left\{{ }^{1} \mathrm{H}\right\}$ NMR spectroscopy are invaluable in detecting the presence of phosphine oxides (see below). Thus characterisation of these complexes relies upon $\mathrm{X}$-ray crystallography. The structures reported of $\left[\left\{\mathrm{BiBr}_{3}\left(\mathrm{Me}_{2} \mathrm{P}\left(\mathrm{CH}_{2}\right)_{2} \mathrm{PMe}_{2}\right)\right\}_{2}\right]$ [329], [ $\left.\left\{\mathrm{BiI}_{3}\left(\mathrm{o}-\mathrm{C}_{6} \mathrm{H}_{4}\left(\mathrm{AsMe}_{2}\right)_{2}\right)\right\}_{2}\right]$ [335] and $\left[\left\{\mathrm{BiCl}_{3}\left(\mathrm{Ph}_{2} \mathrm{P}\left(\mathrm{CH}_{2}\right)_{2} \mathrm{PPh}_{2}\right)_{2}\right\}_{2}\right]$ [339] are the symmetrical dimer type shown above for antimony and comparison of the isomorphous pair $\left[\left\{\mathrm{EBr}_{3}\left(\mathrm{Me}_{2} \mathrm{P}\left(\mathrm{CH}_{2}\right)_{2} \mathrm{PMe}_{2}\right)\right\}_{2}\right](\mathrm{E}=\mathrm{Sb}$ or $\mathrm{Bi})$ [329] reveals very similar geometries, but with the distortions in the bridging unit markedly less in the bismuth case. In contrast the 


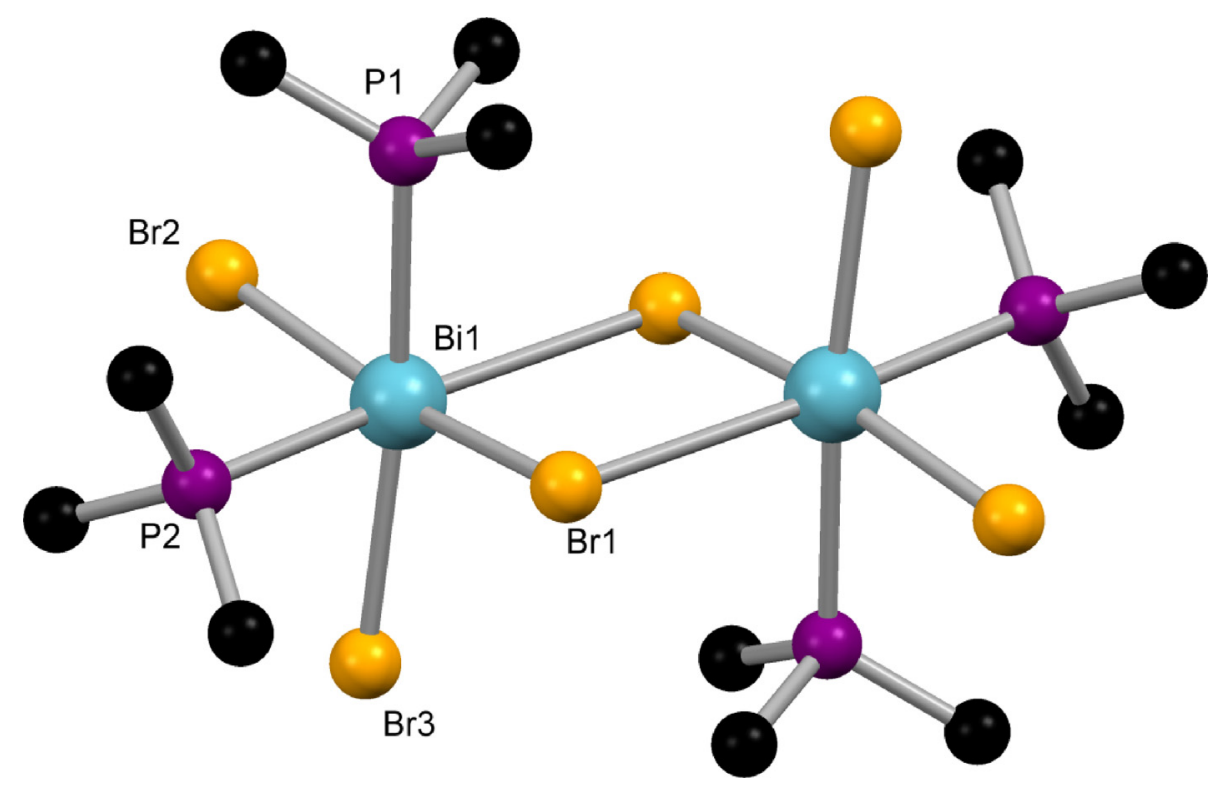

Fig. 62. View of the structure of $\left[\mathrm{Bi}_{2} \mathrm{Br}_{4}(\mu-\mathrm{Br})_{2}\left(\mathrm{PMe}_{3}\right)_{4}\right]$ redrawn from Ref. [336].

diphosphinomethane complex, $\left[\left\{\mathrm{BiCl}_{3}\left(\mathrm{Ph}_{2} \mathrm{PCH}_{2} \mathrm{PPh}_{2}\right)\right\}_{2}\right]$ [339] has a planar $\mathrm{Cl}_{2} \mathrm{Bi}(\mu-\mathrm{Cl})_{2} \mathrm{BiCl}_{2}$ unit with two bridging diphosphines (Fig. 65). Whilst this is a unique structure in Group 15 Lewis acid chemistry, the geometry is driven by the short backbone of the diphosphine ligand and analogues among the early d-block metals are well known [9]. Finally, there is the case of $\left[\mathrm{Bi}_{2} \mathrm{X}_{6}\left(\mathrm{Ph}_{2} \mathrm{P}\left(\mathrm{CH}_{2}\right)_{2} \mathrm{PPh}_{2}\right)_{3}\right][338,339]$. Crystals grown from the reaction of $\mathrm{BiCl}_{3}$ with $\mathrm{Ph}_{2} \mathrm{P}\left(\mathrm{CH}_{2}\right)_{2} \mathrm{PPh}_{2}$ in $\mathrm{MeCN}$ were found to contain equal numbers of molecules of $\left[\left\{\mathrm{BiCl}_{3}\left(\mathrm{Ph}_{2} \mathrm{P}\left(\mathrm{CH}_{2}\right)_{2} \mathrm{PPh}_{2}\right)_{2}\right\}_{2}\right]$ (above) and $\left[\mathrm{Bi}_{2} \mathrm{Cl}_{6}\left(\mathrm{Ph}_{2} \mathrm{P}\left(\mathrm{CH}_{2}\right)_{2} \mathrm{PPh}_{2}\right)_{3}\right]$, which has a centrosymmetric structure composed of two $\mathrm{BiCl}_{3}\left\{\mathrm{\kappa}^{2}-\mathrm{Ph}_{2} \mathrm{P}\left(\mathrm{CH}_{2}\right)_{2} \mathrm{PPh}_{2}\right\}$ units bridged by the third diphosphine (Fig. 66) [339].

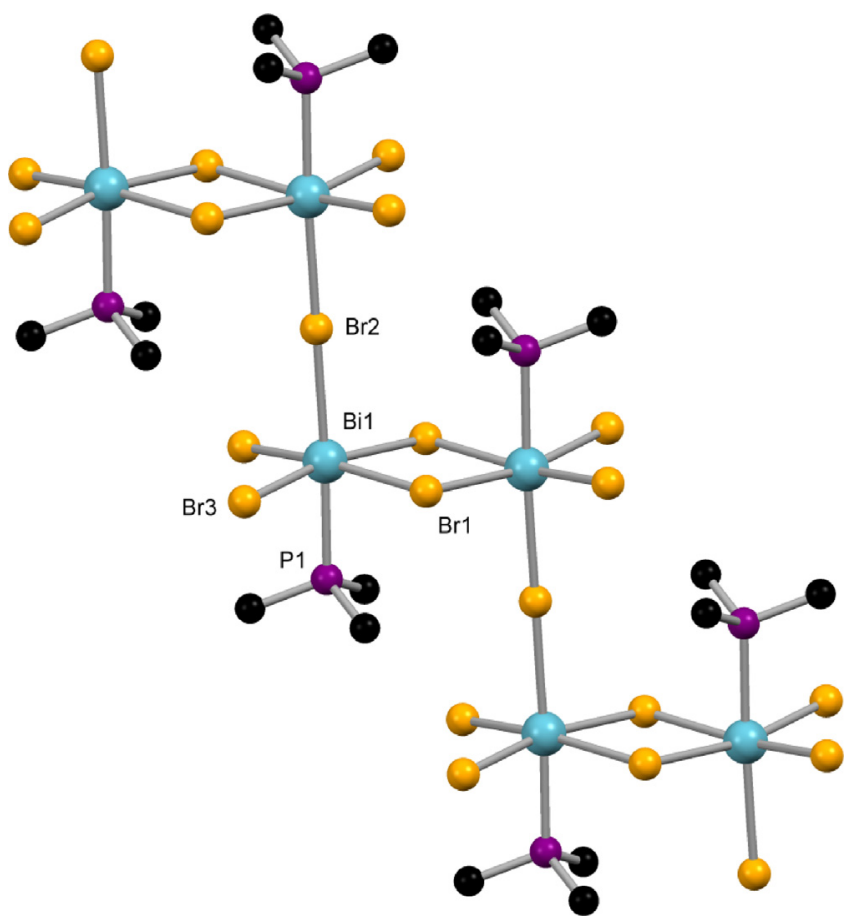

Fig. 63. View of the chain structure of the anion $\left[\mathrm{Bi}_{2} \mathrm{Br}_{7}\left(\mathrm{PMe}_{3}\right)_{2}\right]^{-}$redrawn from Ref. [337].
Air-stable ligands including $\mathrm{Ph}_{2} \mathrm{PCH}_{2} \mathrm{PPh}_{2}, \mathrm{Ph}_{2} \mathrm{As}\left(\mathrm{CH}_{2}\right)_{2} \mathrm{AsPh}_{2}$ and $0-\mathrm{C}_{6} \mathrm{H}_{4}\left(\mathrm{PPh}_{2}\right)_{2}$ air-oxidise readily in the presence of bismuth(III) halides to form complexes of the corresponding ligand dioxides $[335,340]$. The reactions are slower than in the case of $\mathrm{Sn}$ (IV) or Ge(IV) halides (q.v.), and in the bismuth case the mechanism has not been investigated, but it seems plausible that coordination of the ligand to the metal activates the former towards reaction with molecular dioxygen. The structure of $\left[\left\{\mathrm{BiCl}_{3}\left(\mathrm{Ph}_{2} \mathrm{P}(\mathrm{O}) \mathrm{CH}_{2} \mathrm{P}(\mathrm{O}) \mathrm{Ph}_{2}\right)\right\}_{2}\right]$ from crystals obtained in this way shows that the bridging diphosphine in the starting material has been converted to chelating diphosphine dioxide in the product (Fig. 67) [340]. From $\left[\left\{\mathrm{BiCl}_{3}\left(0-\mathrm{C}_{6} \mathrm{H}_{4}\left(\mathrm{PPh}_{2}\right)_{2}\right)\right\}_{2}\right]$ in thf solution, the crystallographically authenticated product was $\left[\mathrm{BiCl}_{3}\left\{0-\mathrm{C}_{6} \mathrm{H}_{4}\left(\mathrm{P}(\mathrm{O}) \mathrm{Ph}_{2}\right)\right\}(\right.$ thf $\left.)\right]$ [335]. The crystals obtained from $\mathrm{BiCl}_{3}$ and $\mathrm{Ph}_{2} \mathrm{As}\left(\mathrm{CH}_{2}\right)_{2} \mathrm{AsPh}_{2}$ were $\left[\mathrm{BiCl}_{3}\left\{\mathrm{Ph}_{2} \mathrm{MeAs}(\mathrm{O})\right\}\left\{\mathrm{Ph}_{2} \mathrm{As}(\mathrm{O})\left(\mathrm{CH}_{2}\right)_{2} \mathrm{As}(\mathrm{O}) \mathrm{Ph}_{2}\right\}\right]$, where in addition to oxidation at arsenic, $\mathrm{C}-\mathrm{C}$ bond cleavage in the ligand backbone has occurred [340]. Whilst the As- $\mathrm{C}$ bonds in $-\mathrm{As}\left(\mathrm{CH}_{2}\right)_{2} \mathrm{As}-$ are known to be susceptible to the elimination of $\mathrm{H}_{2} \mathrm{C}=\mathrm{CH}_{2}$ [3], the apparent cleavage of the strong $\mathrm{C}-\mathrm{C}$ bond is remarkable.

The structures of the triarsine complexes [ $\left.\mathrm{BiX}_{3}\left\{\mathrm{MeC}\left(\mathrm{CH}_{2} \mathrm{AsMe}_{2}\right)_{3}\right\}\right]$ have not been established, but it is likely that they are dimers with the triarsine $\kappa^{2}$-coordinated, a coordination mode found in early transition metal complexes like those of Ti(IV) or V(IV) [341,342].

Bismuth complexes of phosphinothiolate anions are also known [343].

\section{Group 16}

Selenium(IV) and tellurium(IV) halides are reduced by phosphines; the complex mixture of products formed depends upon the reaction conditions, but the primary reaction is the formation of halophosphorus $(\mathrm{V})$ cations $\left[\mathrm{PR}_{3} \mathrm{X}\right]^{+}$and selenium or tellurium anions, sometimes along with elemental Se or $\mathrm{Te}$ [344-346]. In the presence of air and moisture, the major products with $\mathrm{TeX}_{4}$ are protonated phosphine oxide salts of halotellurates(IV) [346]. Careful bromination of phosphine selenides affords $\left.\left[\left(\mathrm{R}_{3} \mathrm{P}\right) \mathrm{SeBr}_{2}\right] \mathrm{R}=\mathrm{NMe}_{2}, \mathrm{NEt}_{2}, \mathrm{Cy},{ }^{i} \mathrm{Pr},{ }^{t} \mathrm{Bu}\right)$ which have $\mathrm{T}$-shaped geometries at $\mathrm{Se}\left(10 \mathrm{e}^{-}\right.$systems $)$and are formally phosphine complexes of $\mathrm{SeBr}_{2}[347,348]$. However, iodination of $\mathrm{R}_{3}$ PSe produces 


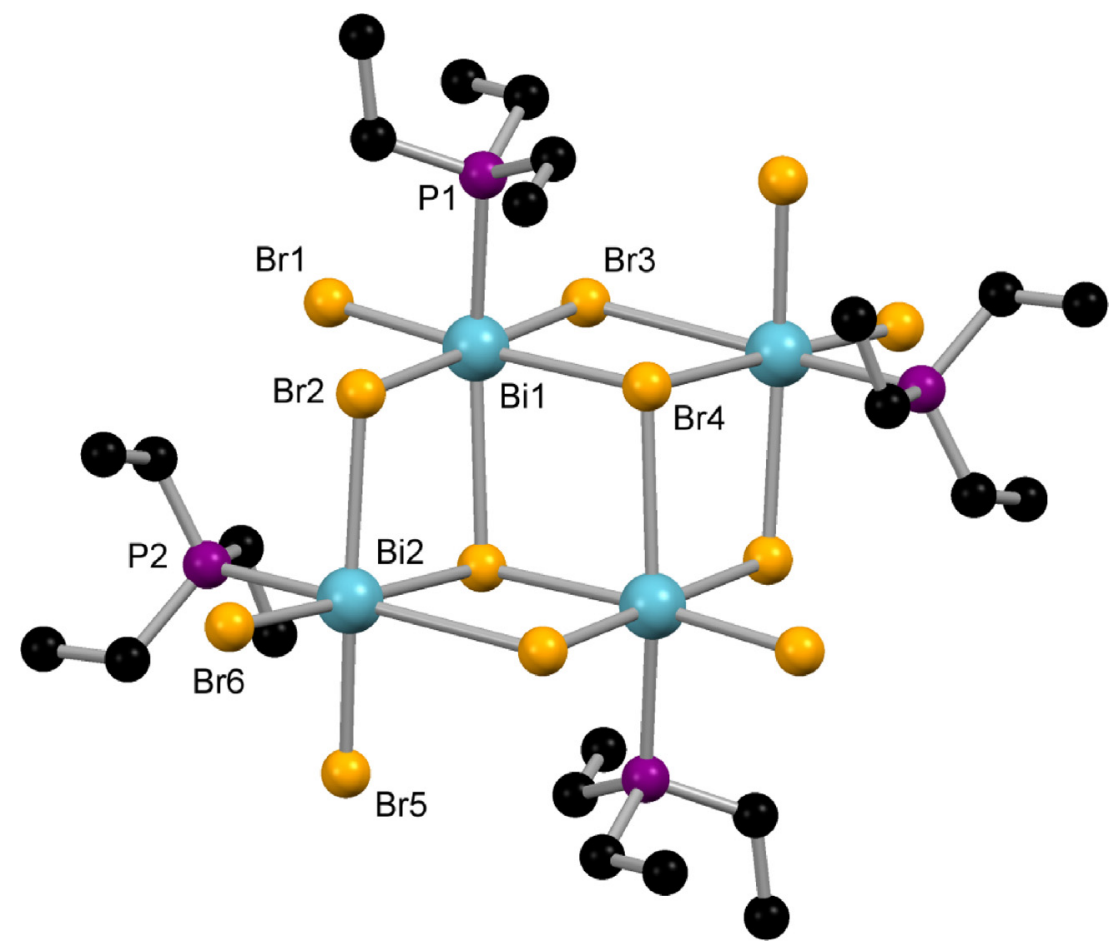

Fig. 64. View of the structure of $\left[\mathrm{Bi}_{4} \mathrm{Br}_{12}\left(\mathrm{PEt}_{3}\right)_{4}\right]$ redrawn from Ref. [329].

the charge transfer complexes $\mathrm{R}_{3}$ PSe-I-I [349,350]. There are similar complexes of both types derived from diphosphine diselenides [351], but the initial products of reaction of $\mathrm{R}_{3} \mathrm{PSe}$ with $\mathrm{Cl}_{2}$ or $\mathrm{SO}_{2} \mathrm{Cl}_{2}$ eliminate elemental selenium below room temperature forming $\mathrm{R}_{3} \mathrm{PCl}_{2}$ [352]. Et ${ }_{3} \mathrm{PTe}$ reacts with $\mathrm{SO}_{2} \mathrm{Cl}_{2}$ or $\mathrm{I}_{2}$ to form [(Et $\left.\left.\mathrm{E}_{3} \mathrm{P}\right) \mathrm{TeX}_{2}\right](\mathrm{X}=\mathrm{Cl}$ or $\mathrm{I})$, and $\left[\left(\mathrm{Et}_{3} \mathrm{P}\right) \mathrm{TeBr}_{2}\right]$ was formed from $\left[\left(\mathrm{Et}_{3} \mathrm{P}\right) \mathrm{TeCl}_{2}\right.$ ] and $\mathrm{Me}_{3} \mathrm{SiBr}$ [353]. All have T-shaped structures at $\mathrm{Te}$ with weak halogen bridges giving centrosymmetric dimer units (Fig. 68). Although they cannot be made directly from selenium or tellurium halides, complexes of $\mathrm{Ph}_{2} \mathrm{E}\left(\mathrm{CH}_{2}\right)_{2} \mathrm{EPh}_{2}$

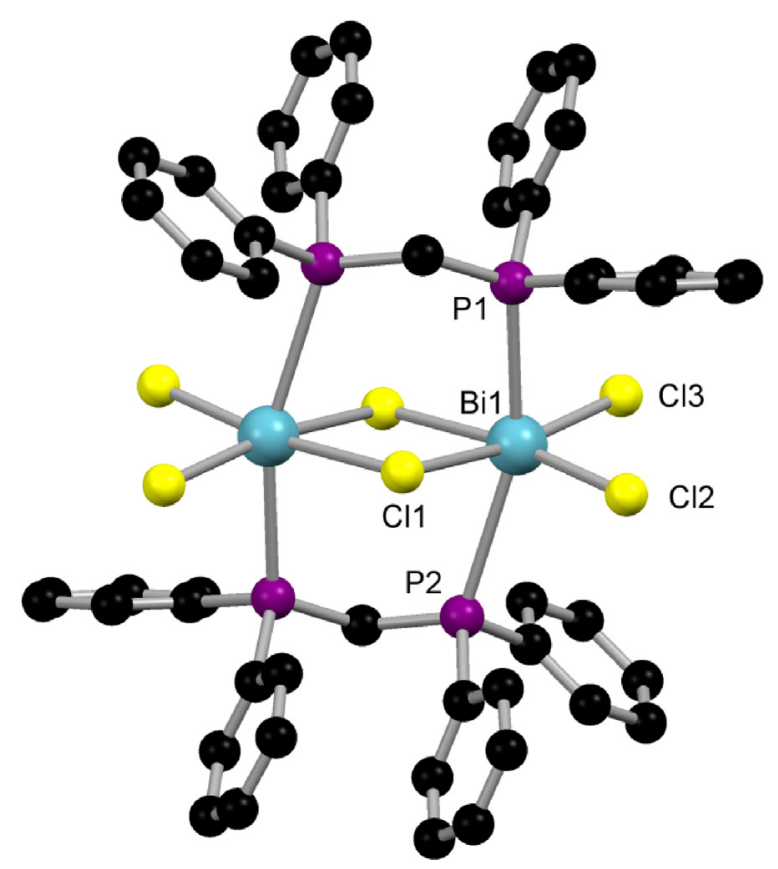

Fig. 65. View of the structure of $\left[\left\{\mathrm{BiCl}_{3}\left(\mathrm{Ph}_{2} \mathrm{PCH}_{2} \mathrm{PPh}_{2}\right)\right\}_{2}\right]$ redrawn from Ref. [339].
$(\mathrm{E}=\mathrm{P}$ or $\mathrm{As})$ of the type $\left[\mathrm{M}\left\{\mathrm{Ph}_{2} \mathrm{E}\left(\mathrm{CH}_{2}\right)_{2} \mathrm{EPh}_{2}\right\}\right]\left[\mathrm{O}_{3} \mathrm{SCF}_{3}\right]_{2}$, which are Group 16 analogues of triphosphenium complexes, can be made by ligand exchange from the diazabutadiene complexes $\left[\mathrm{ML}_{2}\right]\left[\mathrm{O}_{3} \mathrm{SCF}_{3}\right]_{2}\left(\mathrm{~L}=\mathrm{R}_{2}\right.$ diazabutadiene, where $\mathrm{R}=$ cyclohexyl or 2,6diisopropylphenyl) [354].

\section{Applications and outlook}

The majority of the work described in previous sections was aimed at exploring the fundamental chemistry of the p-block elements. Although a few complexes of phosphine substituted bi- or poly-dentates have found specific catalytic applications, e.g. zinc complexes in olefin oxide polymerisations [48-50], it seems unlikely that many catalytic applications will emerge. The major area of application lies in the chemistry related to III-V semi-conductor materials, which has been described in detail in excellent reviews. [149,355,356]. Industry has focused on dual source routes for MOCVD utilising the $\mathrm{MR}_{3}(\mathrm{M}=\mathrm{Al}, \mathrm{Ga}$ or $\mathrm{In})$ and $\mathrm{PH}_{3}$ or $\mathrm{AsH}_{3}$, and less frequently primary or secondary phosphines. This work requires ultrapure $\mathrm{MR}_{3}$, and purification can be achieved by formation of adducts with phosphines or amines, from which the $\mathrm{MR}_{3}$ is subsequently released by heating $[141,146]$. Single source precursors are of interest in specific areas including deposition of thin films, deposition into patterned substrates or formation of nanoparticles. Two main types of reagent have been explored, Lewis acid-Lewis base adducts $\left[\mathrm{MR}_{3}\left(\mathrm{ER}_{3}{ }_{3}\right)\right]$ and phosphido- or arsenido-bridged dimers $\left[R_{2} M\left(\mu-E R_{2}^{\prime}\right)_{2} M_{2}\right]$, of which the latter type (which fall outside the scope of the present article) have proved more successful [355]. For nanoparticles, decomposition in a high boiling inert solvent or in the solid state is often used, hence volatility is not relevant, but cleanliness of the decomposition and crystallinity of the particles are key. The quality/purity of the deposits is critical to their use in electronic devices and minimal carbon or halogen contamination is very important. Detailed descriptions of the application of Group 13 adducts are in the reviews cited, but examples are the preparation of nanocrystalline GaP from thermolysis of 


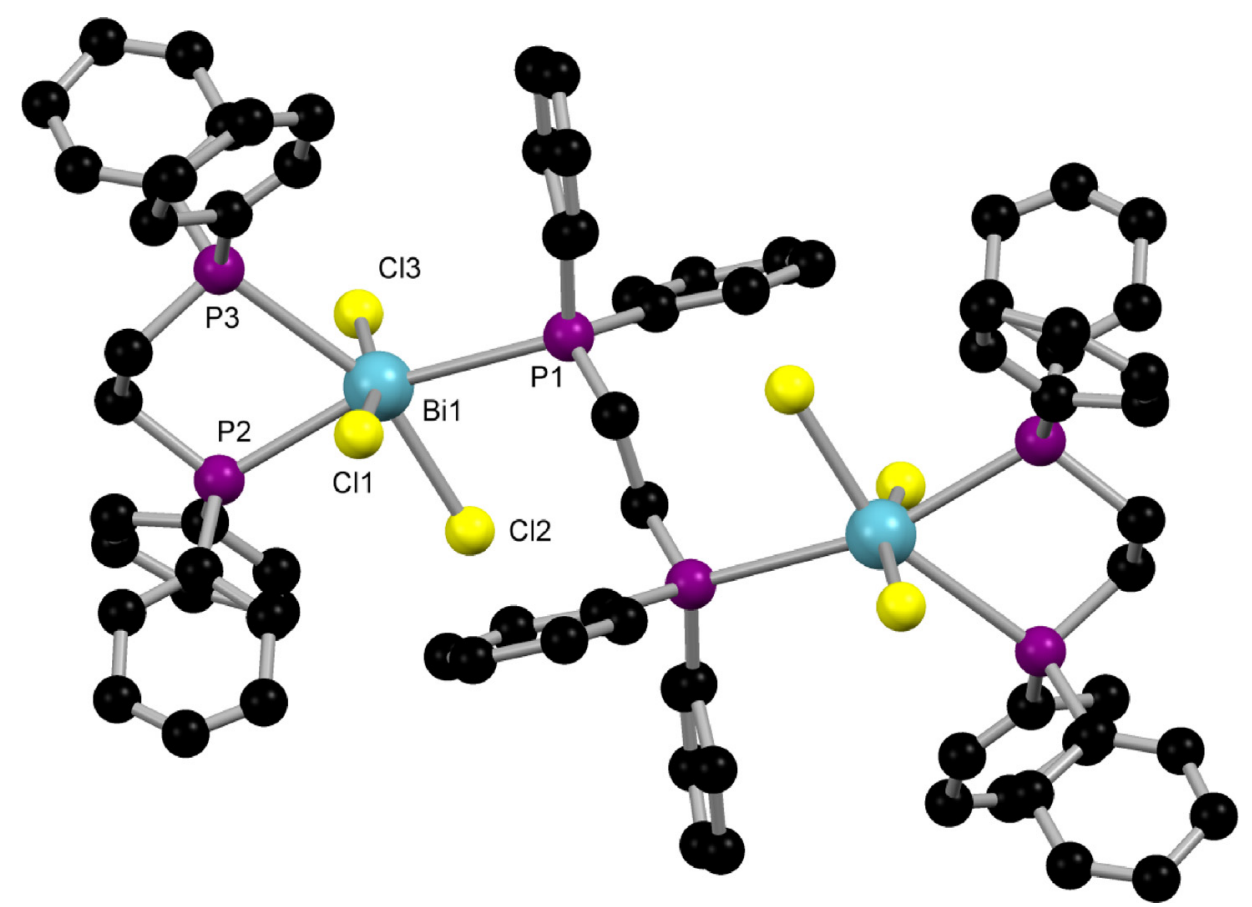

Fig. 66. View of the structure of $\left[\left(\mathrm{BiCl}_{3}\right)_{2}\left\{\mathrm{Ph}_{2} \mathrm{P}\left(\mathrm{CH}_{2}\right)_{2} \mathrm{PPh}_{2}\right\}_{3}\right]$ redrawn from Ref. [339].

$\left[\mathrm{GaX}_{3}\left\{\mathrm{P}\left(\mathrm{SiMe}_{3}\right)_{3}\right\}\right](\mathrm{X}=\mathrm{Cl}, \mathrm{Br}$ or I) [178], and similarly of InP and InAs from [ $\left.\operatorname{InX}_{3}\left\{\mathrm{E}\left(\mathrm{SiMe}_{3}\right)_{3}\right\}\right](\mathrm{E}=\mathrm{P}$ or $\mathrm{As})$ [225] or InSb from $\left[\operatorname{In}^{t} \mathrm{Bu}_{3}\left\{\mathrm{Sb}\left(\mathrm{SiMe}_{3}\right)_{3}\right\}\right][238]$.

Attempts to deposit GeP by AACVD from $\left[\mathrm{GeCl}_{3}(\mathrm{PCyH})\right]$ produced films containing $\mathrm{GeP}$ but these had a very high oxygen content [271], whilst similar use of $\left[\mathrm{SnCl}_{4}\left(\mathrm{AsPh}_{3}\right)_{2}\right]$ produced arsenic-free deposits of $\mathrm{SnO}_{2}$ [285]. Nevertheless these two unsuccessful attempts do not invalidate the idea of utilising this type of complex for the deposition of elements or alloys outside of Ga or In work. Key to its success will be careful choice of ligand and Lewis acid, and for CVD work, temperature control. However, other techniques such as AACVD or electrodeposition from organic solvents do not require volatility, and here the solubility in weakly coordinating solvents and the reduced moisture sensitivity of coordinatively saturated pnictogen ligand complexes may be advantageous.

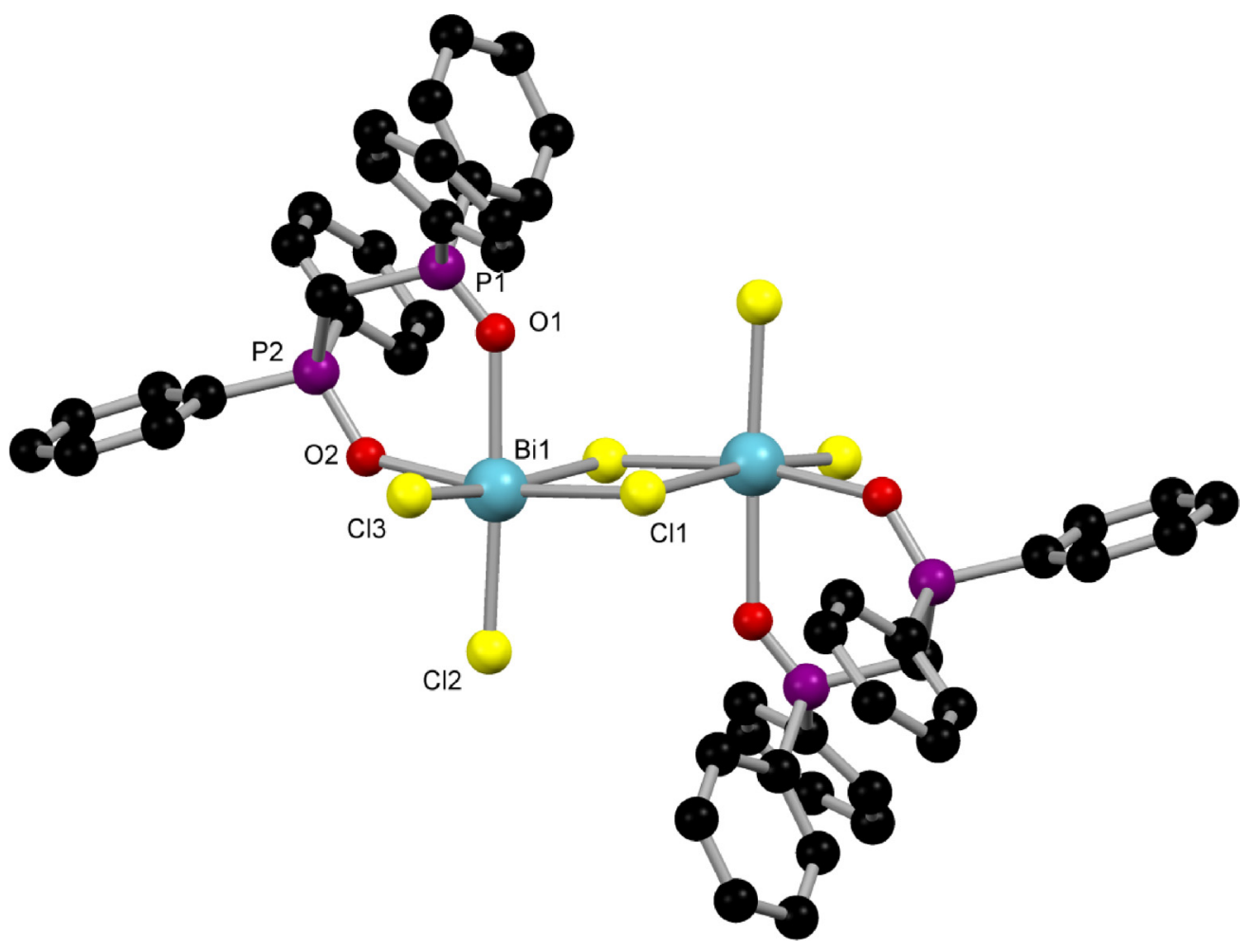

Fig. 67. View of the structure of $\left[\left\{\mathrm{BiCl}_{3}\left(\mathrm{Ph}_{2} \mathrm{P}(\mathrm{O}) \mathrm{CH}_{2} \mathrm{P}(\mathrm{O}) \mathrm{Ph}_{2}\right)\right\}_{2}\right]$ redrawn from Ref. [340]. 


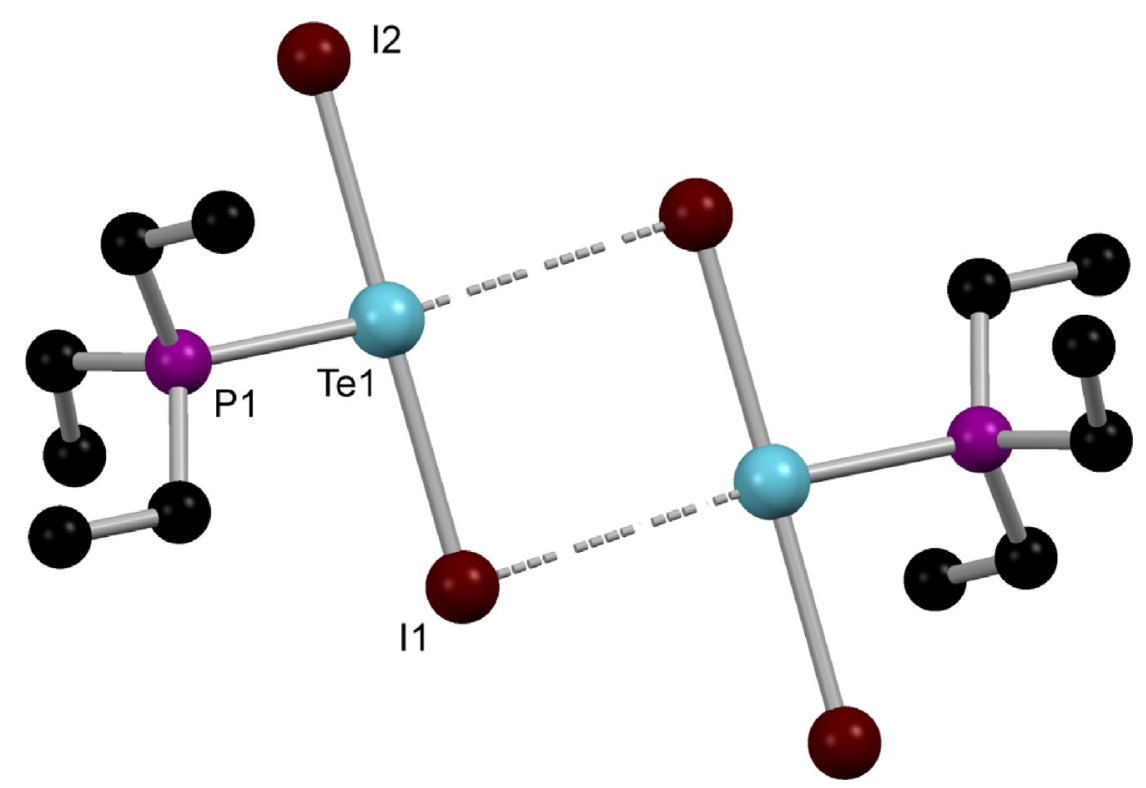

Fig. 68. View of the structure of $\left[\left\{\mathrm{TeI}_{2}\left(\mathrm{PEt}_{3}\right)\right\}_{2}\right]$ redrawn from Ref. [353].

Finally, we note that whilst some areas falling within the scope of this review could be described as well explored, there are others where very little is known. In the latter category are beryllium complexes, no doubt avoided by many workers due to toxicity concerns, and magnesium, for which more examples should be obtainable, although complexes with other s-block elements seem unlikely. In Group 13, Ga and In exhibit a rich chemistry, but aluminium and especially boron are in need of more detailed studies, especially with bi- and poly-dentate ligands. Germanium(II) and (IV) complexes have revealed a surprisingly complicated range of structure types, whilst the very recent studies of phosphine complexes of $\mathrm{Si}(\mathrm{IV})$ halides suggest the possibility that further examples of halosilane complexes may exist. Whilst complexes of thallium(I) or (III) may not form, except in special cases, the lack of effort devoted to lead(II) complexes is remarkable-old results show complexes exist and the syntheses are unlikely to have the moisture sensitivity issues seen elsewhere in Group 14. The Group 15 and 16 element halide chemistry is complicated by weakly bound neutral ligands and problems of associated redox chemistry, but the exciting recent work on phosphenium and arsenium salts show that the area is incompletely explored. The recent characterisation of phosphine complexes of $\mathrm{GeF}_{4}$ and $\mathrm{SnF}_{4}$ show that other hard Lewis acid fluorides and soft pnictogen donor ligands may well be compatible. Another newly developing area is the specific targeting of Lewis acid cations with soft co-ligands, using halide abstractors. Further investigations in this area are likely to emerge.

\section{Acknowledgements}

The figures of X-ray structures were produced using the data deposited in the Cambridge Structural Database. We thank EPSRC for support (JB) under the supercitical fluid project grant $\mathrm{EP} / 1033394$. The SCFED project (www.scfed.net) is a multidisciplinary collaboration of British universities investigating the fundamental and applied aspects of supercritical fluids.

\section{References}

[1] G. Wilkinson, R.D. Gillard, J.A. McCleverty (Eds.), Comprehensive Coordination Chemistry, Pergamon Press, Oxford, 1987.

[2] J.A. McCleverty, T.J. Meyer (Eds.), Comprehensive Coordination Chemistry II, Elsevier, Oxford, 2004.
[3] C.A. McAuliffe, W. Levason, Phosphine, Arsine and Stibine Complexes of the Transition Elements, Elsevier, Amsterdam, 1979

[4] (a) P.W.N.M. van Leeuwen, Homogeneous Catalysis Understanding the Art, Kluwer Academic Publishers, Dordrecht, 2004;

(b) R.H. Crabtree, The Organometallic Chemistry of the Transition Metals, fourth ed., John Wiley \& Sons, 2005.

[5] W. Levason, G. Reid, Coord. Chem. Rev. 250 (2006) 2565.

[6] A.G. Orpen, N.G. Connelly, Organometallics 9 (1990) 1206.

[7] W. Levason, C.A. McAuliffe, Coord. Chem. Rev. 19 (1976) 173.

[8] N.C. Norman, N.L. Pickett, Coord. Chem. Rev. 145 (1995) 27.

[9] W. Levason, in: F.R. Hartley (Ed.), The Chemistry of Organophosphorus Compounds, 1, John Wiley \& Sons, Chichester, 1990, p. 567.

[10] A. Staubitz, A.P.M. Robertson, M.E. Sloan, I. Manners, Chem. Rev. 110 (2010) 4023.

[11] G. Bouhadir, A. Amgoune, D. Bourissou, Adv. Organomet. Chem. 58 (2010) 1.

[12] N.A. Young, Coord. Chem. Rev. 257 (2013) 956.

[13] R.E. Rundle, J. Am. Chem. Soc. 85 (1963) 112.

[14] P. Pyykko, Chem. Rev. 97 (1997) 597.

[15] R.J. Gillespie, I. Hargittai, The VSEPR Model of Molecular Geometry, Allyn and Bacon, Boston, 1991.

[16] G.A. Landrum, R. Hoffmann, Angew. Chem. Int. Ed. 37 (1998) 1887.

[17] G.A. Landrum, N. Goldberg, R. Hoffmann, J. Chem. Soc., Dalton Trans. (1997) 3605.

[18] N.C. Norman, Phosphorus, Sulfur Silicon Relat. Elem. 87 (1994) 167.

[19] J. Emsley, The Elements, O.U.P., Oxford, 1989.

[20] J. Mason (Ed.), Multinuclear NMR, Plenum Press, NY, 1987.

[21] G. Frenking, N. Holzmann, B. Neumüller, K. Dehnicke, Z. Anorg. Allg. Chem. 636 (2010) 1772.

[22] H. Braunschweig, K. Gruss, Z. Naturforsch., B: Chem. Sci. 66 (2011) 55.

[23] G.E. Coates, M. Tranah, J. Chem. Soc. A (1967) 236.

[24] R.A. Kovar, G.L. Morgan, J. Am. Chem. Soc. 92 (1970) 5067.

[25] H. Lehmkuhl, K. Mehler, R. Benn, A. Rufińska, C. Krüger, Chem. Ber. 119 (1986) 1054.

[26] A. Pape, M. Lutz, G. Müller, Angew. Chem. Int. Ed. 33 (1994) 2281.

[27] M. Westerhausen, S. Krieck, J. Langer, T.M.A. Al-Shboul, H. Goerls, Coord. Chem. Rev. 257 (2013) 1049.

[28] P.A.W. Dean, Prog. Inorg. Chem. 24 (1978) 109.

[29] W. Levason, C.A. McAuliffe, C.A. McAuliffe (Eds.), The Chemistry of Mercury, MacMillan Press, London, 1977.

[30] F.A. Cotton, G. Schmid, Polyhedron 15 (1996) 4053.

[31] R.E. DeSimone, G.D. Stucky, Inorg. Chem. 10 (1971) 1808.

[32] F.A. Cotton, S.A. Duraj, W.J. Roth, C.D. Schmulbach, Inorg. Chem. 24 (1985) 525.

[33] S. Vongtragool, B. Gorshunov, M. Dressel, J. Krzystek, D.M. Eichhorn, J. Telser, Inorg. Chem. 42 (2003) 1788.

[34] A.K.S. Chauhan, N. Singh, R.C. Srivastava, Appl. Organomet. Chem. 17 (2003) 856.

[35] H.-F. Klein, J. Montag, U. Zucha, Inorg. Chim. Acta 177 (1990) 43.

[36] N. Bricklebank, S.M. Godfrey, C.A. McAuliffe, A.G. Mackie, R.G. Pritchard, J. Chem. Soc., Chem. Commun. (1992) 944.

[37] S.M. Godfrey, C.A. McAuliffe, R.G. Pritchard, J.M. Sheffield, Inorg. Chim. Acta 292 (1999) 213.

[38] S.M. Godfrey, C.A. McAuliffe, R.G. Pritchard, J.M. Sheffield, J. Chem. Soc., Dalton Trans. (1996) 3309. 
[39] O. Fuhr, D. Fenske, Z. Anorg. Allg. Chem. 625 (1999) 1229.

[40] A. Eichhöfer, D. Fenske, O. Fuhr, Z. Anorg. Allg. Chem. 623 (1997) 762.

[41] J. Eisenmann, D. Fenske, F. Simon, Z. Anorg. Allg. Chem. 621 (1995) 1681

[42] A. Eichhöfer, D. Fenske, H. Pfistner, M. Wunder, Z. Anorg. Allg. Chem. 624 (1998) 1909.

[43] H. Pfistner, D. Fenske, Z. Anorg. Allg. Chem. 627 (2001) 575

[44] D. Zeng, M.J. Hampden-Smith, T.M. Alam, A.L. Rheingold, Polyhedron 13 (1994) 2715.

[45] D. Zeng, M.J. Hampden-Smith, E.M. Larson, Acta Crystallogr., Sect. C: Cryst. Struct. Commun. 50 (1994) 1000.

[46] M. Bochmann, G.C. Bwembya, R. Grinter, A.K. Powell, K.J. Webb, M.B. Hursthouse, K.M.A. Malik, M.A. Mazid, Inorg. Chem. 33 (1994) 2290.

[47] M. Bochmann, G.C. Bwembya, A.K. Powell, X. Song, Polyhedron 14 (1995) 3495.

[48] D.J. Darensbourg, J.R. Wildeson, J.C. Yarbrough, J.H. Reibenspies, J. Am. Chem. Soc. 122 (2000) 12487.

[49] D.J. Darensbourg, M.S. Zimmer, P. Rainey, D.L. Larkins, Inorg. Chem. 39 (2000) 1578.

[50] H.S. Kim, J.Y. Bae, J.S. Lee, O.-S. Kwon, P. Jelliarko, S.D. Lee, S.-H. Lee, J. Catal. 232 (2005) 80.

[51] X. Liu, Y. Liu, Y. Hao, X.-J. Yang, B. Wu, Inorg. Chem. Commun. 13 (2010) 511.

[52] J.H.L. Ong, C. Nataro, J.A. Golen, A.L. Rheingold, Organometallics 22 (2003) 5027.

[53] L.E. Hagopian, A.N. Campbell, J.A. Golen, A.L. Rheingold, C. Nataro, J. Organomet. Chem. 691 (2006) 4890.

[54] E.E. Marlier, S.J. Tereniak, K. Ding, J.E. Milliken, C.C. Lu, Inorg. Chem. 50 (2011) 9290.

[55] J. Dekker, J.W. Muenninghoff, J. Boersma, A.L. Spek, Organometallics 6 (1987) 1236.

[56] P. Perez-Lourido, J. Romero, J.A. Garcia-Vazquez, A. Sousa, K.P. Maresca, J. Zubieta, Inorg Chem. 38 (1999) 3709.

[57] S.-M. Kuang, Z.-Z. Zhang, T.C.W. Mak, J. Chem. Soc., Dalton Trans. (1998) 317.

[58] U. Siemeling, T. Klemann, C. Bruhn, J. Schulz, P. Stepnicka, Dalton Trans. 40 (2011) 4722 .

[59] D. Ellis, L.J. Farrugia, D.T. Hickman, P.A. Lovatt, R.D. Peacock, Chem. Commun. (1996) 1817.

[60] I. D’Auria, M. Lamberti, M. Mazzeo, S. Milione, G. Roviello, C. Pellecchia, Chem. Eur. J. 18 (2012) 2349

[61] J.M. Kessler, J.H. Reeder, R. Vac, C. Yeung, J.H. Nelson, J.S. Frye, N.W. Alcock, Magn. Reson. Chem. 29 (1991) S94.

[62] P. Granger, in: P.S. Pregosin (Ed.), Transition Metal NMR, Elsevier, Amsterdam, 1991, p. 265.

[63] P.A.W. Dean, N.C. Payne, J.J. Vittal, Y. Yu, Inorg. Chem. 32 (1993) 4632.

[64] A. Eichhöfer, A. Aharoni, U. Banin, Z. Anorg. Allg. Chem. 628 (2002) 2415.

[65] D.J. Darensbourg, J.R. Wildeson, J.C. Yarbrough, R.E. Taylor, Inorg. Chem. 40 (2001) 3639.

[66] P.R. Meehan, G. Ferguson, R.P. Shakya, E.C. Alyea, J. Chem. Soc., Dalton Trans. (1997) 3487.

[67] C. Zhu, L. Yang, D. Li, Acta Crystallogr., Sect. E: Struct. Rep. Online 66 (2010) m1586.

[68] (a) N.A. Bell, M. Goldstein, L.A. March, I.W. Nowell, Inorg. Chim. Acta 83 (1984) 75;

(b) R. Colton, D. Dakternieks, Aust. J. Chem. 33 (1980) 1677

[69] G.A. Bowmaker, H.J. Clase, N.W. Alcock, J.M. Kessler, J.H. Nelson, J.S. Frye, Inorg. Chim. Acta 210 (1993) 107.

[70] R. Colton, D. Dakternieks, Aust. J. Chem. 33 (1980) 955.

[71] M.B. Mariyatra, K. Panchanatheswaran, J.N. Low, C. Glidewell, Acta Crystallogr., Sect. C: Cryst. Struct. Commun. 61 (2005) m211.

[72] N.A. Bell, L.A. March, I.W. Nowell, Inorg. Chim. Acta 162 (1989) 57.

[73] N.A. Bell, N.A. Chudley, L.A. March, I.W. Nowell, Inorg. Chim. Acta 141 (1988) 155.

[74] N.W. Bell, L.A. Marsh, I.W. Nowell, Inorg. Chim Acta 179 (1991) 73.

[75] A.N. Kharat, J.B. Tamaddoni, A. Bakhoda, A. Abbasi, J. Coord. Chem. 63 (2010) 3783.

[76] L.J. Baker, G.A. Bowmaker, B.W. Skelton, A.H. White, J. Chem. Soc., Dalton Trans. (1993) 3235

[77] N.A. Bell, S.J. Coles, M.B. Hursthouse, M.E. Light, K.A. Malik, R. Mansor, Polyhedron 19 (2000) 1719.

[78] N.A. Bell, S.J. Coles, C.P. Constable, M.B. Hursthouse, M.E. Light, R. Mansor, N.J. Salvin, Polyhedron 21 (2002) 1845.

[79] G.A. Bowmaker, B. Assadollahzadeh, A.M. Brodie, E.W. Ainscough, G.H. Freeman, G.B. Jameson, Dalton Trans. (2005) 1602.

[80] L.J. Baker, G.A. Bowmaker, P.C. Healey, B.W. Skelton, A.H. White, J. Chem. Soc., Dalton Trans. (1992) 989.

[81] N.K. Jha, J.R. Ugal, S. Singh, Main Group Met. Chem. 16 (1993) 355.

[82] F. Cecconi, C.A. Ghilardi, P. Innocenti, S. Midollini, A. Orlandini, A. Ienco, A. Vacca, J. Chem. Soc., Dalton Trans. (1996) 2821

[83] B. Hoge, C. Thosen, I. Patenburg, Inorg. Chem. 40 (2001) 3084.

[84] J. McGinley, V. McKee, C.J. McKenzie, Acta Crystallogr., Sect. C: Cryst. Struct. Commun 54 (1998) 345.

[85] Y.-Y. Niu, Y.-L. Song, S.-X. Liu, X.-Q. Xin, Z. Anorg. Allg. Chem. 628 (2002) 179.

[86] P. Stepnicka, I. Cisarova, J. Podlaha, J. Ludvik, M. Nejezchleba, J. Organomet. Chem. 582 (1999) 319.

[87] R.R. Guimerans, B.C. Noll, M.M. Olmstead, A.L. Balch, Inorg. Chim. Acta 187 (1991) 181.
[88] W. Oberhauser, T. Stampfl, R. Haid, C. Langes, C. Bachmann, H. Kopacka, K.-H. Ongania, P. Bruggeller, Polyhedron 20 (2001) 727.

[89] F. Cecconi, C.A. Ghilardi, S. Midollini, A. Orlandini, Inorg. Chim. Acta 217 (1994) 155

[90] C.A. Ghilardi, P. Innocenti, S. Midollini, A. Orlandini, A. Vacca, J. Chem. Soc. Chem. Commun. (1992) 1691.

[91] F. Cecconi, C.A. Ghilardi, S. Midollini, A. Orlandini, A. Vacca, Polyhedron 20 (2001) 2885.

[92] P. Barbaro, F. Cecconi, C.A. Ghilardi, S. Midollini, A. Orlandini, A. Vacca, Inorg. Chem. 33 (1994) 6163.

[93] F. Cecconi, C.A. Ghilardi, S. Midollini, A. Orlandini, A. Vacca, J. Organomet Chem. 510 (1996) 153.

[94] F. Cecconi, C.A. Ghilardi, A. Ienco, S. Midollini, A. Orlandini, J Organomet.Chem. 575 (1999) 119.

[95] A. Mühlecker-Knoepfler, E. Ellmerer-Müller, R. Konrat, K.-H. Ongania, K. Wurst, P. Peringer, J. Chem. Soc., Dalton Trans. (1997) 1607.

[96] A. Mühlecker-Knoepfler, B. Scheffter, H. Kopacka, K. Wurst, P. Peringer, J. Chem. Soc., Dalton Trans. (1999) 2525.

[97] B. Scheffter, W. Schuh, K.-H. Ongania, H. Kopacka, R. Malleier, K. Wurst, P. Peringer, Polyhedron 19 (2000) 871.

[98] A. Mühlecker-Knoepfler, W. Schuh, B. Scheffter, H. Lopacka, K. Wurst, P. Peringer, Inorg. Chim. Acta 303 (2000) 70

[99] A. Knoepfler, K. Wurst, P. Peringer, J. Chem. Soc., Chem. Commun. (1995) 131

[100] T.S. Lobana, M.K. Sandhu, M.R. Snow, E.R.T. Tiekink, Acta Crystallogr., Sect. C: Cryst. Struct. Commun. 44 (1988) 179.

[101] M. Kubicki, S.K. Hadjikakou, M.N. Xanthopoulou, Polyhedron 20 (2001) 2179

[102] L. Liu, Q.-F. Zhang, W.-H. Leung, Acta Crystallogr., Sect. E: Struct. Rep. Online $60(2004) \mathrm{m} 394$

[103] X.Q. Wang, W.T. Yu, D. Xu, H.Q. Sun, Acta Crystallogr., Sect. E: Struct. Rep. Online 61 (2005) m548.

[104] I. Pantenburg, C. Thoesen, B. Hoge, Z. Anorg. Allg. Chem. 628 (2002) 1785.

[105] E.C. Alyea, G. Ferguson, R.P. Shakya, P.R. Meehan, Inorg. Chem. 36 (1997) 4749

[106] G. Singh, A.K. Singh, J.E. Drake, M.B. Hursthouse, M.E. Light, Polyhedron 25 (2006) 2915.

[107] A. Staubitz, A.P.M. Robertson, I. Manners, Chem. Rev. 110 (2010) 4079

[108] B.D. Rowsell, R.J. Gillespie, G.L. Heard, Inorg. Chem. 38 (1999) 4659.

[109] F. Bessac, G. Frenking, Inorg. Chem. 42 (2003) 7990.

[110] F. Bessac, G. Frenking, Inorg. Chem. 45 (2006) 6956

[111] T.A. Ford, J. Phys. Chem. A 112 (2008) 7296.

[112] J.A. Plumley, J.D. Evanseck, J. Phys. Chem. A 113 (2009) 5985.

[113] U. Monkowius, S. Nogai, H. Schmidbaur, Dalton Trans. (2003) 987.

[114] D.L. Black, R.C. Taylor, Acta Crystallogr., Sect. B: Struct. Sci. 31 (1975) 1116.

[115] J.P.H. Charmant, C. Fan, N.C. Norman, P.G. Pringle, Dalton Trans. (2007) 114.

[116] (a) C. Aubauer, K. Davidge, T.M. Klapötke, P. Mayer, H. Piotrowski, A. Schulz, Z. Anorg. Allg. Chem. 626 (2000) 2373;

(b) C. Aubauer, G. Engelhardt, T.M. Klapötke, H. Noth, A. Schulz, M. Warchhold, Eur. J. Inorg. Chem. (2000) 2245.

[117] C. Aubauer, T.M. Klapötke, A. Schulz, J. Mol. Struct. THEOCHEM 543 (2001) 285

[118] F. Weller, M. Möhlen, K. Dehnicke, Z. Kristallogr, New Cryst. Struct. 212 (1997) 159.

[119] H. Braunschweig, K. Radacki, K. Uttinger, Inorg. Chem. 46 (2007) 8796.

[120] F. Dornhaus, M. Bolte, H.-W. Lerner, M. Wagner, Eur. J. Inorg. Chem. (2006) 1777.

[121] M.S. Lube, R.L. Wells, P.S. White, Inorg. Chem. 35 (1996) 5007

[122] F. Dornhaus, S. Scholz, I. Sänger, M. Bolte, M. Wagner, H.-W. Lerner, Z. Anorg. Allg. Chem. 635 (2009) 2263.

[123] R.K. Chadha, J.M. Chehayber, J.E. Drake, J. Crystallogr. Spectrosc. Res. 15 (1985) 53.

[124] E. Conrad, J. Pickup, N. Burford, R. McDonald, M.J. Ferguson, Can. J. Chem. 88 (2010) 797.

[125] M.S. Lube, R.J. Jouet, R.L. Wells, P.S. White, V.G. Young Jr., Main Group Chem. 2 (1997) 89.

[126] M.S. Lube, R.L. Wells, P.S. White, Main Group Met. Chem. 19 (1996) 733.

[127] M.S. Lube, R.L. Wells, P.S. White, J. Chem. Soc., Dalton Trans. (1997) 285.

[128] Y. Gushikem, F. Watari, J. Chem. Soc., Dalton Trans. (1980) 2016.

[129] J.E. Drake, L.N. Khasrou, A. Majid, Can. J. Chem. 59 (1981) 2417.

[130] S.L. Benjamin, W. Levason, G. Reid, Chem. Soc. Rev. 42 (2013) 1460.

[131] A.Y. Timoshkin, M. Bodensteiner, T.N. Sevastianova, A.S. Lisovenko, E.I. Davydova, M. Scheer, C. Grassl, A.V. Butlak, Inorg. Chem. 51 (2012) 11602.

[132] A. Ecker, H. Schnöckel, Z. Anorg. Allg. Chem. 624 (1998) 813.

[133] A. Ecker, E. Baum, M.A. Friesen, M.A. Junker, C. Üffing, R. Köppe, H. Schnöckel, Z. Anorg. Allg. Chem. 624 (1998) 513.

[134] G. Menard, D.W. Stephan, J. Am. Chem. Soc. 132 (2010) 1796.

[135] R.L. Wells, A.T. McPhail, J.A. Laske, P.S. White, Polyhedron 13 (1994) 2737.

[136] F.R. Bennett, F.M. Elms, M.G. Gardiner, G.A. Koutsantonis, C.L. Raston, N.K. Roberts, Organometallics 11 (1992) 1457

[137] F.M. Elms, M.G. Gardiner, G.A. Koutsantonis, C.L. Raston, J.L. Atwood, K.D Robinson, J. Organomet. Chem. 449 (1993) 45.

[138] J.L. Atwood, K.W. Butz, M.G. Gardiner, C. Jones, G.A. Koutsantonis, C.L. Raston, K.D. Robinson, Inorg. Chem. 32 (1993) 3482

[139] A.R. Barron, J. Chem. Soc., Dalton Trans. (1988) 3047.

[140] D.A. Wierda, A.R. Barron, Polyhedron 8 (1989) 831.

[141] V.V. Shatunov, A.A. Korlyukov, A.V. Lebedev, V.D. Sheludyakov, B.I. Kozyrkin, V.Y. Orlov, J. Organomet. Chem. 696 (2011) 2238. 
[142] C.N. McMahon, A.R. Barron, J. Chem. Crystallogr. 27 (1997) 195.

[143] G.H. Robinson, M.F. Self, S.A. Sangokoya, W.T. Pennington, J. Crystallogr. Spectrosc. Res. 18 (1988) 285.

[144] M.F. Self, S.A. Sangokoya, W.T. Pennington, G.H. Robinson, J. Coord. Chem. 21 (1990) 301.

[145] S.A. Sangokoya, B. Lee, M.F. Self, W.T. Pennington, G.H. Robinson, Polyhedron 8 (1989) 1497

[146] D.C. Bradley, H. Chudzynska, M.M. Faktor, D.M. Frigo, M.B. Hursthouse, B. Hussain, L.M. Smith, Polyhedron 7 (1988) 1289.

[147] A. Kuczkowski, S. Schulz, M. Nieger, P.R. Schreiner, Organometallics 21 (2002) 1408 .

[148] R.L. Wells, E.E. Foos, A.L. Rheingold, G.P.A. Yap, L.M. Liable-Sands, P.S. White, Organometallics 17 (1998) 2869

[149] S. Schulz, Coord. Chem. Rev. 215 (2001) 1.

[150] S. Schulz, A. Kuczkowski, M. Nieger, J. Organomet. Chem. 604 (2000) 202.

[151] S. Schulz, A. Kuczkowski, M. Nieger, H. Saxell, J. Organomet. Chem. 695 (2010) 2281.

[152] S. Schulz, M. Nieger, Organometallics 18 (1999) 315.

[153] A. Kuczkowski, S. Schulz, M. Nieger, Eur. J. Inorg. Chem. (2001) 2605.

[154] A. Kuczkowski, S. Fahrenholz, S. Schulz, M. Nieger, Organometallics 23 (2004) 3615.

[155] D. Schuchmann, A. Koczkowski, S. Fahrenholz, S. Schulz, U. Flörke, Eur. J. Inorg. Chem. (2007) 931.

[156] R.L. Wells, A.T. McPhail, M.F. Self, J.A. Laske, Organometallics 12 (1993) 3333.

[157] R.L. Wells, A.T. McPhail, T.M. Speer, J. Eur, Solid State Inorg. Chem. 29 (1992) 63.

[158] R.L. Wells, A.T. McPhail, T.M. Speer, Organometallics 11 (1992) 960.

[159] D.A. Atwood, A.H. Cowley, R.A. Jones, R.J. Powell, C.M. Nunn, Organometallics 15 (1996) 2657.

[160] R. Kumar, D.G. Dick, S.U. Ghazi, M. Taghiof, M.J. Heeg, J.P. Oliver, Organometallics 14 (1995) 1601.

[161] G. Müller, J. Lachmann, A. Rufińska, Organometallics 11 (1992) 2970.

[162] G. Müller, J. Lachmann, Z. Naturforsch., B: Chem. Sci. 48 (1993) 1544.

[163] M.D. Healy, D.A. Wierda, A.R. Barron, Organometallics 7 (1988) 2543.

[164] M. Haddad, M. Laghzaoui, R. Welter, S. Dagorne, Organometallics 28 (2009) 4584.

[165] J.-D. Lee, J. Ko, M. Cheong, S.O. Kang, Organometallics 24 (2005) 5845.

[166] M.D. Fryzuk, G.R. Giesbrecht, G. Olovsson, S.J. Rettig, Organometallics 15 (1996) 4832

[167] J.C. DeMott, C. Guo, B.M. Foxman, D.V. Yandulov, O.V. Ozerov, Mendeleev Commun. 17 (2007) 63.

[168] M.D. Fryzuk, G.R. Giesbrecht, S.J. Rettig, Inorg. Chem. 37 (1998) 6928.

[169] M.D. Fryzuk, G.R. Giesbrecht, S.J. Rettig, G.P.A. Yap, J. Organomet. Chem. 591 (1999) 63.

[170] F. Cheng, A.L. Hector, W. Levason, G. Reid, M. Webster, W. Zhang, Inorg. Chem. 46 (2007) 7215

[171] F. Cheng, H.L. Codgbrook, A.L. Hector, W. Levason, G. Reid, M. Webster, W. Zhang, Polyhedron 26 (2007) 4147.

[172] S. Nogai, H. Schmidbaur, Inorg. Chem. 41 (2002) 4770.

[173] L.-J. Baker, L.A. Kloo, C.E.F. Rickard, M.J. Taylor, J. Organomet. Chem. 545-546 (1997) 249.

[174] J.C. Carter, G. Jugie, R. Enjalbert, J. Galy, Inorg. Chem. 17 (1978) 1248.

[175] L.-J. Baker, C.E.F. Rickard, M.J. Taylor, J. Organomet. Chem. 464 (1994) C4.

[176] M.A. Brown, J.A. Castro, D.G. Tuck, Can. J. Chem. 75 (1997) 333.

[177] F. Chen, G. Ma, G.M. Bernard, R.E. Wasylishen, R.G. Cavell, R. McDonald, M.J. Ferguson, Chem. Eur. J. 19 (2013) 2826.

[178] J.F. Janik, R.A. Baldwin, R.L. Wells, W.T. Pennington, G.L. Schimek, A.L. Rheingold, L.M. Liable-Sands, Organometallics 15 (1996) 5385.

[179] M. Sigl, A. Schier, H. Schmidbaur, Eur. J. Inorg. Chem. (1998) 203.

[180] F. Cheng, A.L. Hector, W. Levason, G. Reid, M. Webster, W. Zhang, Acta Crystallogr., Sect. E: Struct. Rep. Online 63 (2007) m1761.

[181] M. Sigl, A. Schier, H. Schmidbaur, Z. Naturforsch., B: Chem. Sci. 53 (1998) 1301.

[182] F. Cheng, A.L. Hector, W. Levason, G. Reid, M. Webster, W. Zhang, Dalton Trans. (2007) 2207.

[183] C.U. Doriat, M. Friesen, E. Baum, A. Ecker, H. Scnöckel, Angew. Chem. Int. Ed. 36 (1997) 1969.

[184] J.M. Slattery, A. Higelin, T. Bayer, I. Krossing, Angew. Chem. Int. Ed. 49 (2010) 3228.

[185] A. Higelin, U. Sachs, S. Keller, I. Krossing, Chem. Eur. J. 18 (2012) 10029.

[186] A. Schnepf, C. Doriat, E. Möllhausen, H. Schnöckel, Chem. Commun. (1997) 2111.

[187] B. Beagley, S.M. Godfrey, K.J. Kelly, S. Kungwankunakorn, C.A. McAuliffe, R.G. Pritchard, Chem. Commun. (1996) 2179.

[188] R.J. Baker, H. Bettentrup, C. Jones, Eur. J. Inorg. Chem. (2003) 2446.

[189] D.H. Mayo, Y. Peng, P. Zavalij, K.H. Bowen, B.W. Eichhorn, Acta Crystallogr., Sect. E: Struct. Rep. Online 68 (2012) m1245.

[190] R.A. Baldwin, E.E. Foos, R.L. Wells, P.S. White, A.L. Rheingold, G.P.A. Yap, Organometallics 15 (1996) 5035.

[191] S. Schulz, A. Kuczkowski, M. Nieger, J. Chem. Crystallogr. 40 (2010) 1163.

[192] S. Schulz, M. Nieger, J. Chem. Soc., Dalton Trans. (2000) 639.

[193] D. Schuchmann, S. Schulz, U. Flörke, Acta Crystallogr., Sect. E: Struct. Rep. Online 63 (2007) m1606.

[194] A. Kuczkowski, S. Schulz, M. Nieger, Appl. Organomet. Chem. 18 (2004) 244.

[195] S. Schulz, M. Nieger, J. Organomet. Chem. 570 (1998) 275

[196] M.A. Banks, O.T. Beachley, J.D. Maloney, R.D. Rogers, Polyhedron 9 (1990) 335.
[197] (a) R.L. Wells, A.T. McPhail, J.W. Pasterczyk, A. Alvanipour, Organometallics 11 (1992) 226;

(b) R.L. Wells, R.A. Baldwin, P.S. White, W.T. Pennington, A.L. Rheingold, G.P.A. Yap, Organometallics 15 (1996) 91.

[198] R.L. Wells, S.R. Aubuchon, M.F. Self, J.P. Jasinski, R.C. Woudenberg, R.J. Butcher, Organometallics 11 (1992) 3370.

[199] J.A. Burns, W.T. Pennington, G.H. Robinson, Organometallics 14 (1995) 1533

[200] A. Kuczkowski, F. Thomas, S. Schulz, M. Nieger, Organometallics 19 (2000) 5758.

[201] R.L. Wells, A.T. McPhail, L.J. Jones III, M.F. Self, R.J. Butcher, Organometallics 11 (1992) 2694

[202] H. Schmidbaur, S. Lauteschläger, G. Müller, J. Organomet. Chem. 281 (1985) 25.

[203] A. Adolf, M. Zabel, M. Scheer, Eur. J. Inorg. Chem. (2007) 2136.

[204] C. Jones, C. Schulten, A. Stasch, Main Group Met. Chem. 28 (2005) 89.

[205] M. Sigl, A. Schier, H. Schmidbaur, Z. Naturforsch. Teil B 53 (1998) 1313.

[206] R.S. Nyholm, K. Ulm, J. Chem. Soc. (1965) 4199.

[207] C.Y. Tang, R.A. Coxall, A.J. Downs, T.M. Greene, L. Kettle, S. Parsons, D.W.H. Rankin, H.E. Robertson, A.R. Turner, Dalton Trans. (2003) 3526.

[208] J.L. Atwood, K.D. Robinson, F.R. Bennett, F.M. Elms, G.A. Koutsantonis, C.L. Raston, D.J. Young, Inorg. Chem. 31 (1992) 2673.

[209] D. O'Hare, J.S. Foord, T.C.M. Page, T.J. Whitaker, J. Chem. Soc., Chem. Commun. (1991) 1445.

[210] F.M. Elms, G.A. Koutsantonis, C.L. Raston, J. Chem. Soc., Chem. Commun. (1995) 1669.

[211] C.Y. Tang, A.J. Downs, T.M. Greene, S. Marchant, S. Parsons, Inorg. Chem. 44 (2005) 7143

[212] H. Schmidbaur, S.D. Nogai, Z. Anorg. Allg. Chem. 630 (2004) 2218.

[213] H. Schumann, F. Girgsdies, B. Heymer, J. Kaufmann, C. Marschall, W. Wassermann, Z. Anorg Allg. Chem. 633 (2007) 2268.

[214] O.T. Beachley, M.A. Banks, M.R. Churchill, W.G. Feighery, J.C. Fettinger, Organometallics 10 (1991) 3036.

[215] A.H. Cowley, C.S. King, A. Decken, Organometallics 14 (1995) 20.

[216] M. Wieber, T. Clarius, Z. Anorg. Allg. Chem. 621 (1995) 1288.

[217] K. Yurkerwich, G. Parkin, Inorg. Chim. Acta 364 (2010) 157.

[218] A.M. Valean, S. Gomez-Ruiz, P. Lonnecke, I. Silaghi-Dumitrescu, L. SilaghiDumitrescu, E. Hey-Hawkins, Inorg. Chem. 47 (2008) 11284.

[219] A.M. Valean, S. Gomez-Ruiz, P. Lonnecke, I. Silaghi-Dumitrescu, L. SilaghiDumitrescu, E. Hey-Hawkins, New J. Chem. 33 (2009) 1771.

[220] J.A.J. Pardoe, A.J. Downs, Chem. Rev. 107 (2007) 2.

[221] M.J. Taylor, D.G. Tuck, L. Victoriano, Can. J. Chem. 60 (1982) 690.

[222] S.M. Godfrey, K.J. Kelly, P. Kramkowski, C.A. McAuliffe, R.G. Pritchard, Chem. Commun. (1997) 1001.

[223] M.V. Veidis, G.J. Palenik, J. Chem. Soc., Chem. Commun. (1969) 586.

[224] W. Clegg, N.C. Norman, N.L. Pickett, Acta Crystallogr., Sect. C: Cryst. Struct. Commun. 50 (1994) 36.

[225] R.L. Wells, S.R. Aubuchon, S.S. Kher, M.S. Lube, P.S. White, Chem. Mater. 7 (1995) 793.

[226] I.A. Degnan, N.W. Alcock, S.M. Roe, M.G.H. Wallbridge, Acta Crystallogr., Sect. C: Cryst. Struct. Commun. 48 (1992) 995.

[227] M.A. Brown, D.G. Tuck, E.J. Wells, Can. J. Chem. 74 (1996) 1535

[228] N.W. Alcock, I.A. Degnan, O.W. Howarth, M.G.H. Wallbridge, J. Chem. Soc. Dalton Trans. (1992) 2775.

[229] (a) F. Chen, G. Ma, R.G. Cavell, V.V. Terskikh, R.E. Wasylishen, Chem. Commun. (2008) 5933;

(b) F. Chen, G. Ma, G.M. Bernard, R.G. Cavell, R. McDonald, M.J. Ferguson, R.E. Wasylishen, J. Am. Chem. Soc. 132 (2010) 5479.

[230] R.E. Wasylishen, K.C. Wright, K. Eichele, T.S. Cameron, Inorg. Chem. 33 (1994) 407.

[231] M. Sigl, A. Schier, H. Schmidbaur, Z. Naturforsch., B: Chem. Sci. 54 (1999) 21.

[232] F. Cheng, S.I. Friend, A.L. Hector, W. Levason, G. Reid, M. Webster, W. Zhang, Inorg. Chem. 47 (2008) 9691.

[233] M. Sigl, A. Schier, H. Schmidbaur, Z. Naturforsch., B: Chem. Sci. 54 (1999) 1417.

[234] D.E. Hibbs, C. Jones, N.A. Smithies, Chem. Commun. (1999) 185.

[235] M.L. Cole, D.E. Hibbs, C. Jones, N.A. Smithies, J. Chem. Soc., Dalton Trans. (2000) 545.

[236] R.J. Baker, M.L. Cole, C. Jones, M.F. Mahon, J. Chem. Soc., Dalton Trans. (2002) 1992.

[237] M.F. Self, A.T. McPhail, L.J. Jones III, R.L. Wells, Polyhedron 13 (1994) 625

[238] E.E. Foos, R.L. Wells, A.L. Rheingold, J. Cluster Sci. 10 (1999) 121.

[239] O.T. Beachley, D.J. MacRae, A.Y. Kovalevsky, Y. Zhang, X. Li, Organometallics 21 (2002) 4632.

[240] P. Visona, F. Benetollo, G. Rossetto, P. Zanella, P. Traldi, J. Organomet. Chem. 511 (1996) 59.

[241] K. Kiyokawa, M. Yasuda, A. Baba, Organometallics 30 (2011) 2039.

[242] K. Saatchi, B.O. Patrick, C. Orvig, Dalton Trans. (2005) 2268.

[243] G.G. Briand, R.J. Davidson, A. Decken, Inorg. Chem. 44 (2005) 9914.

[244] P. Perez-Lourido, J. Romero, J.A. Garcia-Vasquez, A. Sousa, K. Maresca, J. Zubieta, Inorg. Chem. 38 (1999) 1293.

[245] R.A. Baldwin, R.L. Wells, P.S. White, Main Group Chem. 2 (1997) 67.

[246] J.C. DeMott, F. Basuli, U.J. Kilgore, B.M. Foxman, J.C. Huffman, O.V. Ozerov, D.J. Mindiola, Inorg. Chem. 46 (2007) 6271.

[247] J.C. Thomas, J.C. Peters, Inorg. Chem. 42 (2003) 5055.

[248] J.C. Peters, J.C. Thomas, J.A. Dupont, C.G. Riordan, Inorg. Synth. 34 (2004) 8. 
[249] I.R. Shapiro, D.M. Jenkins, J.C. Thomas, M.W. Day, J.C. Peters, Chem. Commun. (2001) 2152.

[250] T.A. Betley, J.C. Peters, Inorg. Chem. 42 (2003) 5074.

[251] C.E. MacBeth, J.C. Thomas, T.A. Betley, J.C. Peters, Inorg. Chem. 43 (2004) 4645 .

[252] C.C. Lu, J.C. Peters, Inorg. Chem. 45 (2006) 8597.

[253] H. Fleischer, Eur. J. Inorg. Chem. (2001) 393.

[254] E.I. Davydova, A.Y. Timoshkin, T.N. Sevastianova, A.V. Suvarov, G. Frenking, J. Mol. Struct. THEOCHEM 767 (2006) 103.

[255] A.Y. Timoshkin, E.I. Davidova, T.N. Sevastianova, A.V. Suvorov, H.F. Schaefer, Int. J. Quantum Chem. 88 (2002) 436.

[256] W. Levason, G. Reid, W. Zhang, Coord. Chem. Rev. 255 (2011) 1319.

[257] O. Holloczki, L. Nyulaszi, Organometallics 28 (2009) 4159.

[258] D.J.D. Wilson, S.A. Couchman, J.L. Dutton, Inorg. Chem. 51 (2012) 7657.

[259] K. George, A.L. Hector, W. Levason, G. Reid, G. Sanderson, M. Webster, W. Zhang, Dalton Trans. 40 (2011) 1584.

[260] I.R. Beattie, G.A. Ozin, J. Chem. Soc. A (1969) 2267

[261] W. Levason, D. Pugh, G. Reid, Inorg. Chem. 52 (2013) 5185.

[262] J. Tillmann, F. Meyer-Wegner, A. Nadj, J. Becker-Baldus, T. Sinke, M. Bolte, M.C. Holthausen, M. Wagner, H.-W. Lerner, Inorg. Chem. 51 (2012) 8599.

[263] F. Cheng, M.F. Davis, A.L. Hector, W. Levason, G. Reid, M. Webster, W. Zhang, Eur. J. Inorg. Chem. (2007) 2488.

[264] M.F. Davis, W. Levason, G. Reid, M. Webster, Dalton Trans. (2008) 2261.

[265] I.R. Beattie, G.A. Ozin, J. Chem. Soc. A (1970) 370.

[266] D.K. Frieson, G.A. Ozin, Can. J. Chem. 51 (1973) 2697.

[267] W.-W. du Mont, H.-J. Kroth, H. Schumann, Chem. Ber. 109 (1976) 3017.

[268] F. Ruthe, W.-W. du Mont, P.G. Jones, Chem. Commun. (1997) 1947.

[269] W.-W. du Mont, Z. Anorg. Allg. Chem. 458 (1979) 85.

[270] S.M. Godfrey, I. Mushtaq, R.G. Pritchard, J. Chem. Soc., Dalton Trans. (1999) 1319.

[271] L. Apostolico, M.F. Mahon, K.C. Molloy, R. Binions, C.S. Blackman, C.J. Carmalt, I.P. Parkin, Dalton Trans. (2004) 470

[272] C. Couret, J. Escudie, H. Ranaivonjatovo, J. Satge, Organometallics 5 (1986) 113.

[273] Y. Inoguchi, S. Okui, K. Mochida, A. Itai, Bull. Chem. Soc. Jpn. 58 (1985) 974.

[274] L.A. Leites, A.V.Zabula, S.S. Bukalov, A.A. Korlyukov, P.S. Koroteev, O.S. Maslennikova, M.P. Egorov, O.M. Nefedov, J. Mol. Struct. 750 (2005) 116.

[275] R.B. King, Inorg. Chem. 2 (1963) 199.

[276] N. Bruncks, W.-W. du Mont, J. Pickardt, G. Rudolph, Chem. Ber. 114 (1981) 3572.

[277] F. Cheng, A.L. Hector, W. Levason, G. Reid, M. Webster, W. Zhang, Inorg. Chem. 49 (2010) 752.

[278] W.-W. du Mont, G. Rudolph, Z. Naturforsch., B: Chem. Sci. 36 (1981) 1215.

[279] A.S. Ionkin, W.J. Marshall, B.M. Fish, Organometallics 25 (2006) 4170.

[280] N.D. Reddy, A. Jana, H.W. Roesky, P.P. Samuel, C. Schulzke, Dalton Trans. (2010) 234.

[281] M.F. Davis, M. Clarke, W. Levason, G. Reid, M. Webster, Eur. J. Inorg. Chem. (2006) 2773.

[282] G.G. Mather, G.M. McLaughlin, A. Pidcock, J. Chem. Soc., Dalton Trans. (1973) 1823.

[283] N. Bricklebank, S.M. Godfrey, C.A. McAuliffe, R.G. Pritchard, Chem. Commun. (1994) 695

[284] E. MacDonald, L. Doyle, S.S. Chitnis, U. Werner-Zwanziger, N. Burford, A. Decken, Chem. Commun. 48 (2012) 7922.

[285] M.F. Mahon, N.I. Moldovan, K.C. Molloy, A. Muresan, I. Silaghi-Dumitrescu, L. Silaghi- Dumistrescu, Dalton Trans. (2004) 4017.

[286] F. Kunkel, K. Dehnicke, H. Goesmann, D. Fenske, Z. Naturforsch., B: Chem. Sci. 50 (1995) 848.

[287] A.R.J. Genge, W. Levason, G. Reid, Inorg. Chim. Acta 288 (1999) 142.

[288] A.S. Batsanov, R.M.K. Deng, K.B. Dillon, A.E. Goeta, J.A.K. Howard, J. Meldrum, P.K. Monks, H. Puschmann, H.J. Shepherd, Heteroat. Chem. 20 (2009) 136.

[289] A.I. Tursina, V.M. Ionov, L.A. Aslanov, S.V. Medvedev, I.B. Zastenker, Zh. Strukt. Khim. 24 (1983) 181.

[290] D. Dakternieks, H. Zhu, E.R.T. Tiekink, Main Group Met. Chem. 17 (1994) 519.

[291] M.M. Ebrahim, H. Stoeckli-Evans, K. Panchanatheswaran, J. Organomet. Chem. 692 (2007) 2168.

[292] N.S. Yashina, E.I. Gefel, A.N. Kosina, V.S. Petrosyan, O.A. Reutov, Koord. Khim. 11 (1985) 1639.

[293] N. Bricklebank, S.M. Godfrey, C.A. McAuliffe, K.C. Molloy, J. Chem. Soc., Dalton Trans. (1995) 1593.

[294] L.V. Kucheruk, F.Y. Akhmadullina, I.P. Goldshtein, E.N. Guryanova, A.S. Gelfond, B.D. Chernokalskii, Zh. Obshch. Khim. 51 (1981) 898.

[295] F. Sarikahya, Synth. React. Inorg. Met.-Org. Chem. 19 (1989) 641.

[296] J.F. Malone, B.E. Mann, Inorg. Nucl. Chem. Lett. 8 (1972) 819.

[297] W. McFarlane, N.H. Rees, Polyhedron 8 (1989) 2047.

[298] O.A. Reutov, V.S. Petrosyan, N.S. Yashina, E.I. Gefel, J. Organomet. Chem. 341 (1988) C31.

[299] M.F. Davis, W. Levason, G. Reid, M. Webster, Polyhedron 25 (2006) 930.

[300] W. Levason, R. Patel, G. Reid, J. Organomet. Chem. 688 (2003) 280.
[301] D. Bansal, A.P. Singhal, R.K. Mittal, Res. J. Chem. Environ. 12 (2008) 35.

[302] C.H. Yoder, L.A. Margolis, J.M. Horne, J. Organomet. Chem. 633 (2001) 33.

[303] N.S. Yashina, E.I. Gefel, V.S. Petrosyan, O.A. Reutov, Dokl. Akad. Nauk SSSR 281 (1985) 1387.

[304] A. Walia, N.D. Sadanani, P.N. Kapoor, R.N. Kapoor, J. Inorg. Nucl. Chem. 42 (1980) 1649.

[305] H. Weichmann, J. Organomet. Chem. 262 (1984) 279.

[306] H. Weichmann, J. Meunier-Piret, M. Van Meerssche, J. Organomet. Chem. 309 (1986) 267.

[307] M. Seibert, K. Merzweiler, C. Wagner, H. Weichmann, J. Organomet. Chem. 650 (2002) 25.

[308] U. Baumeister, H. Hartung, A. Krug, K. Merzweiler, T. Schulz, C. Wagner, H. Weichmann, Z. Anorg. Allg. Chem. 626 (2000) 2185

[309] C. Gurnani, A.L. Hector, E. Jager, W. Levason, D. Pugh, G. Reid, Dalton Trans $42(2013) 8364$.

[310] W.-W. DuMont, B. Neudert, Z. Anorg. Allg. Chem. 441 (1978) 86.

[311] P.A.W. Dean, D.D. Phillips, L. Polensek, Can. J. Chem. 59 (1981) 50.

[312] P.A.W. Dean, Can. J. Chem. 61 (1983) 1795.

[313] I. Objartel, H. Ott, D. Stalke, Z. Anorg. Allg. Chem. 634 (2008) 2373.

[314] U. Baumeister, H. Hartung, K. Jurkschat, A. Tzschach, J. Organomet. Chem. 304 (1986) 107.

[315] N. Froelich, P.B. Hitchcock, J. Hu, M.F. Lappert, J.R. Dilworth, J. Chem. Soc. Dalton Trans. (1996) 1941.

[316] K.A. Clark, T.A. George, T.J. Brett, C.R.I.I. Ross, R.K. Shoemaker, Inorg. Chem. 29 (2000) 2252.

[317] K.A. Clark, T.A. George, Inorg. Chem. 44 (2005) 416.

[318] J. Henning, H. Schubert, K. Eichele, F. Winter, R. Pöttgen, H.A. Mayer, L. Wesemann, Inorg. Chem. 51 (2012) 5787.

[319] L.-C. Liang, Y.-N. Chang, H.-S. Chen, H.M. Lee, Inorg. Chem. 46 (2007) 7587.

[320] P. Rutsch, G. Huttner, Z. Naturforsch., B: Chem. Sci. 57 (2002) 25.

[321] P. Perez-Lourido, J. Romero, J.A. Garcia-Vazquez, A. Sousa, Y. Zheng, J.R. Dilworth, J. Zubieta, J. Chem. Soc., Dalton Trans. (2000) 769.

[322] B.D. Ellis, C.L.B. MacDonald, Coord. Chem. Rev. 251 (2007) 936

[323] P.K. Coffer, K.B. Dillon, Coord. Chem. Rev. 257 (2013) 910.

[324] G. Müller, H.-J. Matheus, M. Winkler, Z. Naturforsch., B: Chem. Sci. 56 (2001) 1155.

[325] N.J. Hill, W. Levason, G. Reid, J. Chem Soc, Dalton Trans. (2002) 1188.

[326] G. Baum, A. Greiling, W. Massa, B.C. Hui, J. Lorberth, Z. Naturforsch., B: Chem. Sci. 44 (1989) 560.

[327] B.D. Ellis, M. Carlesimo, C.L.B. MacDonald, Chem. Commun. (2003) 1946

[328] B.D. Ellis, C.L.B. MacDonald, Inorg. Chem. 43 (2004) 5981.

[329] W. Clegg, M.R.J. Elsegood, V. Graham, N.C. Norman, N.L. Pickett, K. Tavakkoli, J. Chem. Soc., Dalton Trans. (1994) 1743.

[330] E. Conrad, N. Burford, R. McDonald, M.J. Ferguson, J. Am. Chem. Soc. 131 (2009) 5066.

[331] W.Clegg, M.R.J. Elsegood, N.C. Norman, N.L. Pickett, J. Chem. Soc., Dalton Trans. (1994) 1753.

[332] H.J. Breunig, M. Denker, R.E. Schulz, E. Lork, Z. Anorg. Allg. Chem. 624 (1998) 81.

[333] H.J. Breunig, M. Denker, K.H. Ebert, J. Chem. Soc., Chem. Commun. (1994) 875.

[334] W. Clegg, M.R.J. Elsegood, V. Graham, N.C. Norman, N.L. Pickett, J. Chem. Soc., Dalton Trans. (1993) 997.

[335] A.R.J. Genge, N.J. Hill, W. Levason, G. Reid, J. Chem. Soc., Dalton Trans. (2001) 1007.

[336] W. Clegg, R.J. Errington, R.J. Flynn, M.E. Green, D.C.R. Hockless, N.C. Norman, V.C. Gibson, K. Tavakkoli, J. Chem. Soc., Dalton Trans. (1992) 1753.

[337] W. Clegg, R.J. Errington, G.A. Fisher, M.E. Green, D.C.R. Hockless, N.C. Norman, Chem. Ber. 124 (1991) 2457.

[338] G. Alonzo, M. Consiglio, N. Bertazzi, C. Preti, Inorg. Chim. Acta 105 (1985) 51.

[339] G.R. Willey, L.T. Daly, M.G.B. Drew, J. Chem. Soc., Dalton Trans. (1996) 1063.

[340] G.R. Willey, M.D. Rudd, C.J. Samuel, M.G.B. Drew, J. Chem. Soc., Dalton Trans. (1995) 759 .

[341] R. Hart, W. Levason, B. Patel, G. Reid, Eur. J. Inorg. Chem. (2001) 2927.

[342] B.M. Gray, A.L. Hector, W. Levason, G. Reid, M. Webster, W. Zhang, M. Jura Polyhedron 29 (2010) 1630.

[343] P. Perez-Lourido, L. Valencia, J. Romero, J.A. Garcia-Vazquez, A. Sousa, J. Zubieta, Polyhedron 45 (2012) 200.

[344] J.L. Dutton, R. Tabeshi, M.C. Jennings, A.J. Lough, P.J. Ragogna, Inorg. Chem. 46 (2007) 8594

[345] S.M. Kunnari, R. Oilunkaniemi, R.S. Laitainen, M. Ahlgrén, J. Chem. Soc., Dalton Trans. (2001) 3417.

[346] S.M. Närhi, R. Oilunkaniemi, R.S. Laitinen, M. Ahlgrén, Inorg. Chem. 43 (2004) 3742.

[347] S.M. Godfrey, S.L. Jackson, C.A. McAuliffe, R.G. Pritchard, J. Chem. Soc., Dalton Trans. (1998) 4201.

[348] C.G. Hrib, F. Ruthe, E. Seppaelae, M. Bätcher, C. Druckenbrodt, C. Wismach, P.G. Jones, W.-W. du Mont, V. Lippolis, F.A. Devillanova, M. Bühl, Eur. J. Inorg. Chem. (2006) 88.

[349] S.M. Godfrey, S.L. Jackson, C.A. McAuliffe, R.G. Pritchard, J. Chem. Soc., Dalton Trans. (1997) 4499

[350] P.D. Boyle, S.M. Godfrey, Coord. Chem. Rev. 223 (2001) 265. 
[351] C.G. Hrib, P.G. Jones, W.-W. du Mont, V. Lippolis, F.A. Devillanova, Eur. J. Inorg. Chem. (2006) 1294.

[352] E. Krawczyk, A. Skowronska, J. Michalski, J. Chem. Soc., Dalton Trans. (2002) 4471.

[353] J. Konu, T. Chivers, Dalton Trans. (2006) 3941.
[354] J.W. Dube, M.M. Hänninen, J.L. Dutton, H.M. Tuononen, P.J. Ragogna, Inorg. Chem. 51 (2012) 8897.

[355] P. O'Brien, N.L. Pickett, in: J.A. McCleverty, T.J. Meyer (Eds.), Comprehensive Coordination Chemistry II, Elsevier, Oxford, 2004, p. 1005.

[356] M.A. Malik, M. Afzaal, P. O’Brien, Chem. Rev. 110 (2010) 4417. 